\title{
Adaptive Fault-Tolerant Control of Multivariable Systems with Applications to NASA Generic Transport Model
}

\begin{tabular}{c} 
A Dissertation \\
Presented to \\
the Faculty of the School of Engineering and Applied Science \\
University of Virginia \\
\hline
\end{tabular}

In Partial Fulfillment

of the Requirements for the Degree

Doctor of Philosophy (Electrical Engineering)

by

JiAXING GuO

August 2013 


\section{APPROVAL SHEET}

The dissertation

is submitted in partial fulfillment of the requirements

for the degree of

Doctor of Philosophy

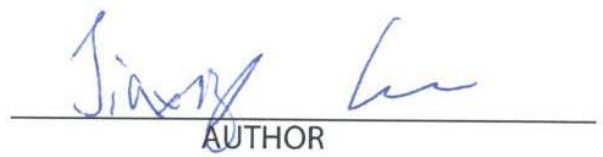

The dissertation has been read and approved by the examining committee:

\begin{tabular}{c} 
Prof. Gang Tao \\
\hline $\begin{array}{c}\text { Advisor } \\
\text { Prof. Zongli Lin }\end{array}$ \\
\hline Prof. Stephen G. Wilson \\
\hline Prof. Maite Brandt-Pearce \\
\hline Prof. Carl R. Knospe
\end{tabular}

Accepted for the School of Engineering and Applied Science:

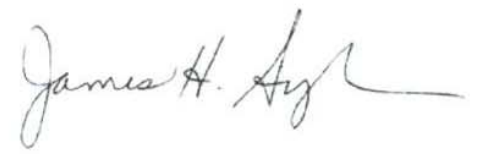

Dean, School of Engineering and Applied Science

August

2013 


\section{Abstract}

For performance-critical systems, such as aircraft flight systems, structural damage, actuator failures, actuator nonlinearities, sensor uncertainties, and environmental disturbances may lead to severe accidents if not promptly and properly mitigated, since adverse conditions can cause large unknown variations of system dynamics, introduce undesired disturbance inputs, and limit the performance of feedback control.

In this research, we will develop novel adaptive fault-tolerant control and faultdetection schemes for multi-input and multi-output (MIMO) systems with structural damage and component failures (such as actuator failures, actuator nonlinearities, and sensor uncertainties) to guarantee desired and safe system performance.

To handle complexities and uncertainties of nonlinear system dynamics, we use linearization-based design methods, where control schemes are developed for linearized system models, with both continuous-time and discrete-time control designs being considered. To accommodate uncertain damage and failures for the MIMO systems, the multivariable model reference adaptive control (MRAC) design method is employed. A key design condition-system infinite zero structure is investigated for both continuous-time linearized models and discrete-time linearized models before and after the adverse conditions occur, and invariance of this essential condition can be concluded under realistic failure and damage conditions. With such an invariance property, some novel fault-tolerant state feedback for output tracking and output feedback for output tracking multivariable MRAC schemes, whose plant-model matching conditions are much less restrictive than that of the state feedback for state tracking design, are developed to ensure stability and asymptotic output tracking for systems in the presence of parametric and structural uncertainties caused by damage and component failures. 
Equipped with the developed feedback adaptive fault-tolerant control to ensure system signal boundedness requirements, novel adaptive fault detectors are constructed based on system dynamic coupling features and different failure patterns to identify and isolate damage and failures in order to enhance situational awareness for control personnel.

The developed adaptive fault-tolerant control and fault detection designs have been evaluated on a high-fidelity aircraft Matlab/Simulink model-the nonlinear NASA generic transport model (GTM), which offers a realistic representation of the aircraft. Extensive simulation studies have been conducted and simulation results have demonstrated the desired performance of our developed designs. 


\section{Acknowledgments}

I would like to gratefully and sincerely thank my advisor, Professor Gang Tao, for his guidance, understanding, patience, and most importantly, persistent encouragement and continuous support during my graduate studies at the University of Virginia. His deep knowledge of control theories and his impeccable attitude towards research have greatly helped me form a rigorous, dedicated, and creative research style for my future career life in the automatic control field.

I am grateful to Dr. Suresh M. Joshi, NASA Langley Research Center, for his helpful comments and suggestions on my research work, especially the simulation studies on the NASA generic transport model. I would also like to thank Professor Zongli Lin, Professor Stephen G. Wilson, Professor Maite Brandt-Pearce, and Pro-

fessor Carl R. Knospe for serving on my Ph.D. advisory committee and providing valuable suggestions to improve my Ph.D. studies.

It is a blessing to be surrounded by many colleagues and friends at the University of Virginia. I am particularly grateful to Qian Sang, Yu Liu, and Thummaros Rugthum for their stimulating discussions and help, and for making my life at U.Va. enjoyable.

I also gratefully appreciate the financial support provided by NSF under grant ECS0601475 and by NASA Langley Research Center under grant NNX08AB99A.

I want to thank my parents without whom this dissertation would not have been possible and meaningful. This dissertation is dedicated to my family.

JiAXING GUO Charlottesville, Virginia 


\section{Contents}

1 Introduction $\quad 2$

1.1 Research Motivation ................... . . 3

1.2 Background and Literature Overview . . . . . . . . . . . . . 4

1.2.1 Fault-Tolerant Control and Fault Detection . . . . . . . . . 4

1.2.2 Model Reference Adaptive Control . . . . . . . . . . . . . . . 6

1.2.3 Digital Control Design Frameworks . . . . . . . . . . . 8

1.3 Dissertation Outline . . . . . . . . . . . . . . . . 10

2 Problem Formulation and Preliminaries 12

2.1 The Aircraft Flight Systems with Adverse Onboard Conditions . . . . 13

2.2 Linearization-Based Design . . . . . . . . . . . . . . . . . 18

2.3 Linear System Preliminaries . . . . . . . . . . . . . . . . . . 19

2.3.1 Infinite Zero Structure . . . . . . . . . . . . . . . . 19

2.3.2 Gain Matrix Decomposition ............. 20

2.4 Output Plant-Model Matching Control . . . . . . . . . . . . . . 22

2.4.1 State Feedback Control Design . . . . . . . . . . . . 22

2.4.2 Output Feedback Control Design . . . . . . . . . . . . . . . . 32

3 Multivariable State Feedback Output Tracking MRAC 34

3.1 Problem Statement . . . . . . . . . . . . . . . . . 35 
3.1 .1 Motivation . . . . . . . . . . . . . . . . 35

3.1 .2 Design Assumptions . . . . . . . . . . . . . . . . . . . 39

3.2 Controller Structure . . . . . . . . . . . . . . . . . . . . . . 39

3.2.1 Robustness to Parameter Uncertainties . . . . . . . . . . . 40

3.2 .2 An Aircraft Example . . . . . . . . . . . . . . . . . . . . 42

3.3 Parameter Adaptation Scheme . . . . . . . . . . . . . . . . . 43

3.3.1 Design Based on the LDS Decomposition . . . . . . . . . . 44

$3.3 .2 \quad$ Stability Analysis . . . . . . . . . . . . . . . 46

3.4 Control Issues of Nonlinear Systems . . . . . . . . . . . . . . . 48

3.4 .1 Control Design . . . . . . . . . . . . . . . . . . 48

3.4 .2 System Infinite Zero Structure . . . . . . . . . . . . . 50

3.5 Application to the GTM . . . . . . . . . . . . . . . 51

3.5.1 The NASA Generic Transport Model (GTM) . . . . . . . . 51

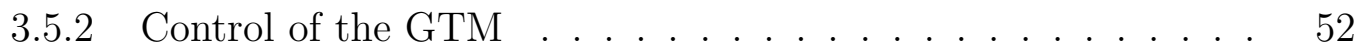

4 Adaptive Structural Damage Compensation for MIMO Systems 59

4.1 Problem Statement . . . . . . . . . . . . . . . . . . . . . . . . . 59

4.2 System Invariance of the Aircraft Model . . . . . . . . . . . . . 64

4.3 The Multivariable MRAC Scheme . . . . . . . . . . . . . . . . . 69

4.4 Simulation Study for the GTM . . . . . . . . . . . . 74

4.4.1 Control of the Linearized GTM Model . . . . . . . . . . 75

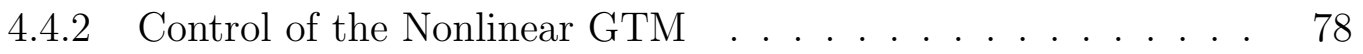

5 Adaptive Simultaneous Actuator Failure and Structural Damage $\begin{array}{lr}\text { Compensation for MIMO Systems } & 84\end{array}$

5.1 Problem Statement . . . . . . . . . . . . . . . . . . . . 85 
5.2 System Invariance of An Aircraft Model _ . . . . . . . . . . . . 89

5.2.1 Generic Structures of the System Parameters . . . . . . . . 90

5.2 .2 Invariance of Infinite Zero Structure . . . . . . . . . . . . . 92

5.3 Adaptive Control Scheme . . . . . . . . . . . . . . . . . . . . 94

5.4 Application to the NASA GTM . . . . . . . . . . . . 100

5.4.1 Control of the Linearized GTM _ . . . . . . . . . . . 101

5.4 .2 Control of the Nonlinear GTM _ . . . . . . . . . . 101

6 Adaptive Sensor Uncertainty Compensation for MIMO Systems 108

6.1 Problem Statement . . . . . . . . . . . . . . . . . . . . . . . . . . . 109

6.2 Adaptive Compensation Scheme . . . . . . . . . . . . . . . . . . . . . 112

6.3 Simulation Study . . . . . . . . . . . . . . . . . . . . . . . . 119

7 Discrete-Time Multivariable Adaptive Control of MIMO Systems with Structural Damage $\quad 124$

7.1 Problem Statement . . . . . . . . . . . . . . . . . . . . . . . 125

7.2 System Infinite Zero Structures _ . . . . . . . . . . . . . 128

7.2.1 Linearized Discrete-Time System Model . . . . . . . . . . 128

7.2.2 Interactor Matrix of Linearized Discrete-Time Model . . . . 132

7.3 A Multivariable MRAC Scheme . . . . . . . . . . . . . . 137

7.4 Application to the NASA GTM . . . . . . . . . . . . 148

7.4.1 Invariance of Infinite Zero Structure of the Aircraft System under Structural Damage . . . . . . . . . . . . . . . . . 149

7.4.2 Simulation Study for the NASA GTM _ . . . . . . . 150

8 Adaptive Output Feedback Actuator Nonlinearity Compensation for $\begin{array}{ll}\text { MIMO Systems } & 157\end{array}$ 
8.1 Problem Statement . . . . . . . . . . . . . . . . . . . . . . . . 158

8.2 Adaptive Actuator Nonlinearity Compensation . . . . . . . . . . . . 161

8.2.1 Adaptive Controller Parametrization . . . . . . . . . . . . 162

8.2 .2 Parameter Adaption Scheme . . . . . . . . . . . . . . . . . 164

8.2 .3 Stability . . . . . . . . . . . . . . . . 168

8.3 Aircraft Flight Control Simulation Study . . . . . . . . . . . . 170

9 Adaptive State Feedback Disturbance Rejection for MIMO Linear $\begin{array}{ll}\text { Time-Invariant Systems } & 175\end{array}$

9.1 Problem Statement . . . . . . . . . . . . . . . . . . . . . . . 175

9.2 Nominal State Feedback Control Design _ . . . . . . . . . . . 177

9.3 Adaptive State Feedback Control Design . . . . . . . . . . . . . 186

9.4 Rejection of Output Disturbance and Constant Dynamics Offset . . . 190

9.4.1 Nominal State Feedback Control Design _ . . . . . . . . . 190

9.4.2 Adaptive State Feedback Control Design . . . . . . . . . . . 193

10 Adaptive Output Feedback Disturbance Rejection for MIMO Piece$\begin{array}{ll}\text { wise Linear Systems } & 195\end{array}$

10.1 Problem Statement . . . . . . . . . . . . . . . . . . 195

10.2 Output Tracking Error Identity _ . . . . . . . . . . . 197

10.3 Adaptive Output Feedback Control Design . . . . . . . . . . . . . 199

11 Feedback-Based Adaptive Damage Detection for MIMO Systems 203

11.1 Problem Statement . . . . . . . . . . . . . . . . . . . . . 204

11.2 Modeling of Aircraft Systems with Damage . . . . . . . . . . . 205

11.3 Feedback-Based Damage Detection Scheme . . . . . . . . . . . . 209

11.3.1 Self-Stabilization Feedback Control . . . . . . . . . . . . 209 
11.3.2 Adaptive Damage Detection . . . . . . . . . . . . . . . 212

11.4 Application to the GTM . . . . . . . . . . . . . 220

11.4.1 Simulation Study for the Linearized GTM . . . . . . . . . . . 220

11.4.2 Simulation Study for the Nonlinear GTM . . . . . . . . . . . . 222

12 Feedback-Based Adaptive Sensor Uncertainty Detection 230

12.1 Problem Statement . . . . . . . . . . . . . . . . . . 231

12.2 Feedback-Based Sensor Uncertainty Detection Scheme . . . . . . . . . 233

12.2.1 Self-stabilization Feedback Control Design . . . . . . . . . . . 234

12.2.2 Sensor Uncertainty Detection Designs . . . . . . . . . . . . 238

12.3 Simulation Study . . . . . . . . . . . . . . . . . . . . 244

13 Conclusions $\quad 250$

$\begin{array}{ll}\text { Bibliography } & 252\end{array}$ 


\section{List of Figures}

2.1 A benchmark aircraft model: the NASA GTM. . . . . . . . . . 18

2.2 Linearization-based fault-tolerant control and fault detection. . . . . . 19

3.1 Closed-loop system with nonlinear system. . . . . . . . . . . . . 49

3.2 System responses vs. reference $\left(r(t)=r_{1}(t)\right) \ldots \ldots \ldots \ldots \ldots$

3.3 System responses vs. reference $\left(r(t)=r_{2}(t)\right) \ldots \ldots \ldots \ldots \ldots$

3.4 GTM outputs (adaptive and fixed) vs. reference. . . . . . . . . 57

4.1 Closed-loop nonlinear aircraft control system with damage. . . . . . . 63

4.2 Linearized GTM output $\Delta y(t)$ (solid) vs. $\Delta y_{m}(t)($ dotted $)\left(r(t)=r_{1}(t)\right) .77$

4.3 Linearized GTM output $\Delta y(t)$ (solid) vs. $\Delta y_{m}(t)$ (dotted) $\left(r(t)=r_{2}(t)\right) .78$

4.4 Responses of the GTM: pitch $\theta(t)$, yaw $\psi(t)$, and roll $\phi(t)$ (Case I). . 79

4.5 Control surface positions: elevator $d_{e}(t)$ and rudder $d_{r}(t)$ (Case I). . 80

4.6 Elements of controller parameters $K_{1}(t), K_{2}(t)$, and $k_{3}(t)$ (Case I). . 80

4.7 Responses of the GTM: pitch $\theta(t)$, yaw $\psi(t)$, and roll $\phi(t)$ (Case II). . 81

4.8 Control surface positions: elevator $d_{e}(t)$ and rudder $d_{r}(t)$ (Case II). $\quad 82$

4.9 Elements of controller parameters $K_{1}(t), K_{2}(t)$, and $k_{3}(t)$ (Case II). . 82

5.1 Linearized GTM outputs vs. reference signals (case I). . . . . . . 102

5.2 Linearized GTM outputs vs. reference signals (case II). . . . . . . 102

5.3 GTM outputs (solid) vs. reference outputs (dotted) (case I) . . . . 103 
5.4 Deflections of four elevator segments (case I). . . . . . . . . . . . . 104

5.5 Deflections of two rudder segments (case I) . . . . . . . . . . . . . . 104

5.6 GTM outputs (solid) vs. reference outputs (dotted) (case II). . . . . . 105

5.7 Deflections of four elevator segments (case II) . . . . . . . . . . . . 105

5.8 Deflections of two rudder segments (case II) . . . . . . . . . . . 106

6.1 Control system with sensor uncertainties. . . . . . . . . . . . . 111

6.2 Aircraft output $y(t)$ (solid) vs. reference $y_{m}(t)$ (dotted) (Case I) . . . . 121

6.3 Compensator output $\hat{y}(t)$ (solid) vs. $y_{m}(t)$ (dotted) (Case I). . . . . . 122

6.4 Aircraft output $y(t)$ (solid) vs. reference $y_{m}(t)$ (dotted) (Case II). . . 122

6.5 Compensator output $\hat{y}(t)$ (solid) vs. $y_{m}(t)$ (dotted) (Case II). . . . . 123

7.1 Digital control of a nonlinear system with damage. . . . . . . . . 126

7.2 pitch $\theta(k T)$, yaw $\psi(k T)$, and roll $\phi(k T)$ (Case I). . . . . . . . . . . 152

7.3 elevator $d_{e}(k T)$ and rudder $d_{r}(k T)$ (Case I) . . . . . . . . . . . . . 153

7.4 pitch $\theta(k T)$, yaw $\psi(k T)$, and roll $\phi(k T)$ (Case II) . . . . . . . . . . 154

7.5 elevator $d_{e}(k T)$ and rudder $d_{r}(k T)$ (Case II) . . . . . . . . . . . 155

8.1 Aircraft outputs (solid) vs. reference outputs (dotted) (case i) . . . . 171

8.2 Commanded control input signal $v(t)$ (case i) . . . . . . . . . . . 172

8.3 Plant input signal $u(t)$ and feedback control signal $u_{d}(t)$ (case i) . . . 172

8.4 Aircraft outputs (solid) vs. reference outputs (dotted) (case ii) . . . . 173

8.5 Commanded control input signal $v(t)$ (case ii) . . . . . . . . . . 173

8.6 Plant input signal $u(t)$ and feedback control signal $u_{d}(t)$ (case ii) . . . 174

11.1 Linearized GTM outputs (solid) vs. reference outputs (dotted). . . . 222

11.2 Detector residuals $\Delta e_{m 8}^{(1)}$ and $\Delta e_{m 8}^{(2)} \ldots \ldots \ldots \ldots \ldots \ldots$

11.3 GTM responses: pitch $\theta(t)$, yaw $\psi(t)$, and roll $\phi(t)$ (case I). . . . . . 224 
11.4 Control surface positions: elevator $d_{e}(t)$ and rudder $d_{r}(t)$ (case I). . . 225

11.5 Detector residuals $\Delta e_{m 8}^{(1)}$ and $\Delta e_{m 8}^{(2)}$ (case I) . . . . . . . . . 225

11.6 GTM responses: pitch $\theta(t)$, yaw $\psi(t)$, and roll $\phi(t)$ (case II). . . . . . 226

11.7 Control surface positions: elevator $d_{e}(t)$ and rudder $d_{r}(t)$ (case II). . . 226

11.8 Detector residuals $\Delta e_{m 8}^{(1)}$ and $\Delta e_{m 8}^{(2)}$ (case II) . . . . . . . . . . . 227

11.9 GTM responses: pitch $\theta(t)$, yaw $\psi(t)$, and roll $\phi(t)$ (case III). . . . . 228

11.10Control surface positions: elevator $d_{e}(t)$ and rudder $d_{r}(t)$ (case III). . 229

11.11Detector residuals $\Delta e_{m 8}^{(1)}$ and $\Delta e_{m 8}^{(2)}$ (case III) . . . . . . . . . . . 229

12.1 Residual $e_{x}(t)=z_{m}(t)-z_{x}(t)$ for the estimation model (i) . . . . . . 247

12.2 Residual $e_{x}(t)=z_{m}(t)-z_{x}(t)$ for the estimation model (ii). . . . . . . 247

12.3 Residual $e_{x}(t)=z_{m}(t)-z_{x}(t)$ for the estimation model (iii). . . . . . 248

12.4 Residual $e_{x}(t)=z_{m}(t)-z_{x}(t)$ for the estimation model (iv). . . . . . 248 


\section{Chapter 1}

\section{Introduction}

System faults may cause large deterioration of system performance and even lead to catastrophic accidents. In order to avoid and mitigate the severe consequences of system faults, system onboard techniques of fault-tolerant control, fault detection, and diagnosis, are urgently required to enhance system reliability and safety. So it is of great importance to develop powerful and effective feedback control algorithms to accommodate and diagnose the system faults for performance-critical systems such as aircraft flight systems. In this research, some novel and effective adaptive control and adaptive estimation techniques will be derived to compensate and detect adverse conditions, such as structural damage, actuator failures, actuator nonlinearities, environmental disturbances, and sensor uncertainties. The developed adaptive fault-tolerant and fault detection schemes can guarantee system stability and enhance situational awareness of control personnel in the presence of system faults, which will be verified and validated on a high-fidelity aircraft model-the NASA generic transport model (GTM) with different fault conditions. 


\section{$1.1 \quad$ Research Motivation}

Adverse onboard conditions of the aircraft flight system, such as airframe damage, actuator failures, actuator nonlinearities, sensor uncertainties, can largely deteriorate flight performance, since the airframe damage causes uncertain structural and parametric variations of the aircraft system dynamics, the failed actuators introduce undesired control inputs, and the actuator nonlinearities and sensor failures can have severe impacts on the feedback control performance. In the aviation history, it has been shown that the airframe damage and component failures are some significant contributors to accidents and fatalities, such as accidents of A330 Air France 2009 and A330 Qantas Flight 2008 due to sensor malfunctions, accident of Convair 580 Air Tahoma 2008 due to elevator system failure, accident of Embraer 120 American Eagle 2000 due to jammed stabilizer, accident of B747 Japan Air Lines 1985 due to airframe damage, etc. [12].

Reliable fault-tolerant control and fault detection schemes are required to guarantee the safety of aircraft systems and enhance the situational awareness ability of the pilots or flight control personnel. A challenge of successful fault compensation and detection is the large structural and parametric uncertainties caused by unknown component failures and damage, whose onset time instants, patterns and severity are all unforeseen. Adaptive methodologies are capable of autonomously compensate and detect system fault conditions when unknown changes in the system dynamics occur. These unique features provide potential to improve flight safety when component failures and airframe damage occur. In this research, we will develop new adaptive control and adaptive detection schemes for aircraft flight systems with large structural and parametric uncertainties. Besides considering continuous-time adaptive control designs, we will further develop discrete-time adaptive fault-tolerant control schemes 
used for constructing digital control system frameworks, since the digital control is widely used in safety-critical systems due to certain advantages over conventional analog control, such as capability of implementing complicated control algorithms and no degradation of performance caused by wear or aging.

Another important aspect of the fault-tolerant control and fault detection designs is verification and validation of the developed schemes on an aircraft flight system under adverse onboard conditions. Since flight test of a full scale manned transport aircraft in adverse flight conditions is difficult and dangerous, we may use a subscale high-fidelity aircraft model to assess our proposed designs. The NASA GTM is a 5.5\% dynamically scaled twin-turbine powered test aircraft for NASA's Airborne Subscale Transport Aircraft Research flight test facility [64], which can play an important role in testing research control laws in adverse flight conditions including damage and failures. In this research, we will implement our designs to a high-fidelity aircraft Matlab/Simulink model of the NASA GTM, which can represent realistic flight scenarios under some uncertain failure and damage conditions, to verify the effectiveness of our adaptive fault-tolerant and fault detection designs.

\subsection{Background and Literature Overview}

In this section, we will present an overview of research on fault-tolerant control, fault detection, adaptive control, and digital control, which provides solid technical foundations for this research.

\subsubsection{Fault-Tolerant Control and Fault Detection}

Considerable effort has been devoted to development of fault-tolerant control and fault detection algorithms for performance-critical applications, particularly aircraft flight

systems. For systems with actuator failures, typical control design methodologies 
include multiple model, switching and tuning schemes $[8,9,30,32,70]$ and adaptive schemes $[1,6,7,78,98]$. Reconfigurable flight control designs using neural networks for aircraft systems with failures have been developed in $[10,13,49,52,74,91]$ and $[15,96,97]$ presented the reconfigurable control designs based on fault conditions detected by adaptive estimators. For systems with parametric and structural uncertainties caused by airframe damage, [41] developed a robust control scheme, and several adaptive compensation designs have been proposed in $[11,37,54,55,57,72,80]$. Adaptive control designs without explicitly detecting faults have also been derived to compensate the actuator failures $[19,56,85,87]$.

In this research, we will develop multivariable model reference adaptive control schemes for the aircraft system with both actuator failures and structural damage to guarantee that the aircraft can track a desired trajectory before and after hazardous conditions occur. For sensor failure accommodation, most methods need to detect and isolate the failed sensors first, before the healthy sensor signals can be used in feedback control. One open problem is that many existing detection algorithms require the control system remains stable in the presence of failures. Therefore, in this research, we will develop adaptive sensor failure compensation schemes to ensure the signal boundedness requirement.

Fault detection and diagnosis problems have also been studied intensively for safety-critical systems. One of the common approaches is to use model-based method $[45,47]$, where detectors are established to estimate the system signals. By observing residuals between the detector model and the system, detection criteria can be derived to identify the faults. A lot of effort has been devoted to the model-based aircraft actuator and sensor failure detection and diagnosis, for example, [4,92] developed aircraft actuator and sensor failure detection and isolation schemes based 
on an adaptive unknown input observer approach, [31] used a hierarchy of techniques to detect and isolate system faults based on a jet aircraft model, [63] used $H_{\infty}$ optimization-based method to make the observer-based actuator fault detection scheme robust, $[17,95]$ built adaptive state observers based on the known system parameters to detect actuator faults, and $[16,28,66,94]$ were based on neural networks and analytical sensor redundancy. Some effort has been work on the model-based damage detection problem, for example, [51] developed a structural damage monitoring scheme by exploiting the analogy between residual force due to stiffness change and input error. For the damage detection problem, others used data-based method by analyzing response of external stimulation signals, such as acoustic analysis [21], vibration-based method $[79,86]$, etc. Since the model-based method only requires signals from the aircraft flight control system such as state and control input signals, while the data-based method needs additional signal excitation equipments, in this research, we apply the model-based method for the aircraft component failure and structural damage detection.

The construction of the model-based detector models requires that the aircraft signals are bounded. Unlike most fault detection schemes operating under the system signal boundedness assumption before and after faults occur, the detection schemes in this research are equipped with fault-tolerant controllers accommodating the adverse onboard conditions and ensuring all the signals of the closed-loop system are bounded.

\subsubsection{Model Reference Adaptive Control}

Model reference adaptive control (MRAC) is an important adaptive control approach that provides feedback controller structures and adaptive laws for control of systems with parametric and structural uncertainties to ensure signal boundedness of the closed-loop system and asymptotic tracking of desired reference signals. Such adaptive 
control designs are desirable for the aircraft flight system to achieve a safe flight and track a desired trajectory in the presence of adverse onboard conditions.

Considerable effort has been devoted to the development of MRAC (e.g., [2, 20, $24,44,46,53,58,65,73,76,84,90])$. While MRAC theory has evolved into a mature branch of control theory, refinements and new designs of MRAC schemes are still needed for many applications, especially, for multi-input and multi-output (MIMO) systems, such as aircraft systems. Several important issues are still open in this area.

Both state feedback and output feedback control designs can be applied to the MRAC scheme. In many applications such as flight control systems, state signals are available for measurement, so that state feedback control design is used due to its simpler structure (as compared with compensator-based output feedback design). State feedback control systems can be designed for either state tracking or output tracking. To develop an adaptive state feedback controller, it is necessary to first solve the related nonadaptive control problem assuming the plant parameters are known, so that an ideal fixed state feedback controller can be obtained. This ideal (nominal) controller will be used as a part of the prior knowledge in the design of the adaptive control scheme. The existence of such a nominal controller is equivalent to a set of matching equations. The state feedback for state tracking design has restrictive matching conditions, which are difficult to be satisfied in the presence of system uncertainties. State feedback for output tracking, on the other hand, while keeping the simple controller structure, needs less restrictive matching conditions. Therefore, adaptive state feedback control for output tracking has a high potential for the aircraft system with parametric and structural uncertainties, where the matching conditions can be satisfied under system parametric and structural uncertainties caused by component failures and structural damage. Research in adaptive state feedback control 
for output tracking has been reported in the literature. In [48], the state feedback for output tracking control is studied for certain classes of nonlinear systems. In [84], a state feedback for output tracking MRAC scheme for single input single output systems is derived. However, the multivariable state feedback for output tracking MRAC problem still needs to be solved. In this research, the adaptive fault-tolerant control designs will be mainly developed based on the multivariable state feedback for output tracking MRAC scheme. A key design condition for the multivariable MRAC-infinite zero structure is investigated for both continuous-time MIMO systems and discretetime MIMO systems before and after the adverse conditions occur, and invariance of this essential condition can be concluded under realistic failure and damage conditions. With such an invariance property, some novel fault-tolerant state feedback for output tracking and output feedback for output tracking multivariable MRAC schemes, whose plant-model matching conditions are much less restrictive than that of the state feedback for state tracking design, are developed to ensure stability and asymptotic output tracking for systems in the presence of parametric and structural uncertainties caused by damage and component failures.

\subsubsection{Digital Control Design Frameworks}

Digital control is widely used in safety-critical systems such as aircraft flight systems due to certain advantages over conventional analog control. For fault-tolerant control, a digital control scheme may be constructed either by discretizing a continuous-time control law or by designing a discrete-time control law based on a discretized system model. In this research, besides developing continuous-time adaptive control schemes, we will also design direct discrete-time adaptive control algorithms for constructing the digital control frameworks.

Much effort has been devoted to developing discrete-time control schemes for non- 
linear systems. [93] studied discretized nonlinear models of continuous-time nonlinear systems and zero dynamics of the discretized nonlinear models. [25,26,29] investigated the dynamic decoupling problems for the discrete-time nonlinear systems and [27] analyzed feedback linearizability for discretized models of continuous-time nonlinear systems. In $[69,71]$, stabilization conditions of discretized nonlinear systems are analyzed. In [5,60,61], feedback linearization control schemes were investigated for the sampled-data nonlinear systems. However, the feedback linearization control designs may not be suitable for dealing with highly complicated nonlinear dynamics, especially systems with parametric and structural uncertainties, such as the nonlinear aircraft flight systems under structural damage conditions. In this research, to deal with complexities and uncertainties of the discrete-time nonlinear dynamics obtained by discretizing the continuous-time nonlinear system with damage, we employ a linearization-based control design. Due to system parametric and structural uncertainties caused by damage, equilibrium points are not available for linearization. Thus, an arbitrary operating point is chosen to linearize the discrete-time nonlinear model before and after damage occurs, which leads to a linearized discrete-time model with unknown system parameters and dynamics offset.

The key design condition for the multivariable MRAC scheme-invariance of the infinite zero structure needs to be satisfied for the linearized discrete-time model before and after damage occurs. In this research, a new investigation of the infinite zero structure of the linearized discrete-time system will be conducted. Based on a thorough study of generic structures of the linearized discrete-time model, we will conclude that, when the discretization sampling period is sufficiently small, the infinite zero structure of the linearized discrete-time model is invariant, no matter what the relative degrees of the continuous-time nonlinear systems are. This property suggests 
that even if the damage changes the relative degree of the continuous-time nonlinear system, the infinite zero structure of the linearized discrete-time model is invariant before and after damage occurs. Based on such an invariance property, we can develop a discrete-time multivariable MRAC scheme to ensure asymptotic output tracking of a common reference system chosen according to the invariant infinite zero structures before and after damage occurs. Hence, we can build a digital control system framework, consisting of the developed linearization-based discrete-time adaptive control law, zero-order holds, and samplers, for the continuous-time nonlinear systems.

\subsection{Dissertation Outline}

This dissertation is organized as follows, where the major results have been documented and published in the journal and conference papers :

In Chapter 2, the fault-tolerant control and fault detection problems for MIMO nonlinear aircraft systems with structural damage, actuator failures, actuator nonlinearities, and sensor uncertainties are formulated. Moreover, some important adaptive control design preliminaries are presented.

In Chapter 3, the multivariable state feedback for output tracking MRAC scheme, which is a foundation of the adaptive fault-tolerant control designs in this research, is developed for MIMO linear time-invariant systems with parametric uncertainties, where the plant-model matching condition is much less restrictive than that of the state feedback for state tracking design.

In Chapter 4-Chapter 8, continuous-time and discrete-time adaptive fault-tolerant control designs are developed to compensate the structural damage, actuator failures, actuator nonlinearities, and sensor uncertainties, which are designed based on the multivariable state feedback and output feedback MRAC design frameworks. 
In Chapter 9 and Chapter 10, we will deal with the uncertain disturbance rejection problems, where an adaptive state feedback disturbance rejection scheme is developed for MIMO linear time-invariant systems and an adaptive output feedback disturbance rejection scheme is developed for MIMO piecewise linear systems.

In Chapter 11 and Chapter 12, adaptive fault detection schemes are developed for diagnosing the structural damage and sensor uncertainties, where the aircraft flight system is equipped with developed fault-tolerant control to stabilize the system before and after hazardous conditions occur.

These adaptive fault-tolerant control and fault detection algorithms have been applied to the high-fidelity nonlinear GTM Matlab/Simulink model, and the simulation results, which are presented in corresponding chapters, can verify the effectiveness of the proposed designs on the aircraft flight systems.

In Chapter 13, we make a conclusion of this dissertation work and provide some recommendations for future research. 


\section{Chapter 2}

\section{Problem Formulation and Preliminaries}

In this chapter, we will formulate the fault-tolerant control and fault detection design framework including essential technical issues of aircraft systems with structural damage, actuator failures, actuator nonlinearities, and sensor uncertainties, basic ideas of how to address these issues, and some key design preliminaries. 


\subsection{The Aircraft Flight Systems with Adverse On- board Conditions}

The dynamic model of a nonlinear aircraft flight system $[11,77]$ is constructed by force, moment and kinematic equations:

$$
\begin{aligned}
\dot{u}_{b} & =(X+T) / m-g \sin \theta+r_{b} v_{b}-q_{b} w_{b}+\Delta f_{1}, \\
\dot{w}_{b} & =Z / m+g \cos \theta \cos \phi+q_{b} u_{b}-p_{b} v_{b}+\Delta f_{2}, \\
I_{y} \dot{q}_{b} & =M-\left(I_{x}-I_{z}\right) p_{b} r_{b}+I_{x z}\left(p_{b}^{2}-r_{b}^{2}\right)+\Delta f_{3}, \\
\dot{\theta} & =q_{b} \cos \phi-r_{b} \sin \phi \\
\dot{v}_{b} & =Y / m+g \cos \theta \sin \phi-r_{b} u_{b}+p_{b} w_{b}+\Delta f_{4}, \\
I_{z} \dot{r}_{b}+I_{x z} \dot{p}_{b} & =N+I_{x z} q_{b} r_{b}+\left(I_{z}-I_{y}\right) q_{b} p_{b}+\Delta f_{5}, \\
I_{x} \dot{p}_{b}+I_{x z} \dot{r}_{b} & =L+\left(I_{z}-I_{y}\right) q_{b} r_{b}-I_{x z} q_{b} p_{b}+\Delta f_{6}, \\
\dot{\phi} & =p_{b}+\tan \theta\left(q_{b} \sin \phi+r_{b} \cos \phi\right), \\
\dot{\psi} & =\frac{q_{b} \sin \phi+r_{b} \cos \phi}{\cos \theta}
\end{aligned}
$$

where $\Delta f_{i}, i=1,2, \ldots, 6$, characterize the structural variations under airframe damage conditions, $u_{b}, v_{b}$ and $w_{b}$ are the body-axis velocity components of the origin of the body-axis frame, $p_{b}, q_{b}$ and $r_{b}$ are the body-axis components of the angular velocity, $\phi, \theta$ and $\psi$ are the Euler roll, pitch and yaw angles of the aircraft body axes with respect to the reference axes, $m$ is the mass of the aircraft, $I_{x}, I_{y}$ and $I_{z}$ are the moments of inertia about body axes, $I_{x z}$ is the cross-product of inertia, $X, Y, Z$, $L, M, N$, and $T$ are the aerodynamic forces, moments, and engine thrust, which are functions of the state signals and the control surface deflections (elevator $d_{e}$, aileron $d_{a}$, and rudder $d_{r}$ ), and the engine throttle $d_{t}$. We can denote the nonlinear dynamics 
$(2.1 .1)-(2.1 .9)$ as

$$
\dot{x}(t)=f(x(t))+g(x(t)) u(t), y(t)=C x(t),
$$

where the state signal is $x(t)=\left[u_{b}, w_{b}, q_{b}, \theta, v_{b}, r_{b}, p_{b}, \phi, \psi\right]^{T}$, the control input signal is $u(t)=\left[d_{e}, d_{t}, d_{a}, d_{r}\right]^{T}$, and the output signal $y(t)$ is chosen as a linear combination of the state signals.

Uncertain changes under damage conditions. When damage occurs, the functions of aerodynamic forces $X, Y, Z$, moments $L, M, N$, and engine thrust $T$ undergo uncertain parametric and structural variations. Furthermore, for the aircraft dynamics model (2.1.1)-(2.1.9), when there is no damage, $\Delta f_{i}=0, i=1,2, \ldots, 6$; after asymmetric damage occurs, the center of mass $\left[\Delta x_{b}, \Delta y_{b}, \Delta z_{b}\right]^{T}$ and the crossproducts of inertial $I_{x y}$ and $I_{y z}$ become non-zero, which result in uncertain structural changes for the dynamics model (2.1.1)-(2.1.9), such that $[11,57]$

$$
\begin{aligned}
\Delta f_{1}= & \left(q_{b}^{2}+r_{b}^{2}\right) \Delta x_{b}-\left(p_{b} q_{b}-\dot{r}_{b}\right) \Delta y_{b}-\left(p_{b} r_{b}+\dot{q}_{b}\right) \Delta z_{b} \\
\Delta f_{2}= & \left(\dot{q}_{b}-p_{b} r_{b}\right) \Delta x_{b}-\left(\dot{p}_{b}+q_{b} r_{b}\right) \Delta y_{b}+\left(p_{b}^{2}+q_{b}^{2}\right) \Delta z_{b} \\
\Delta f_{3}= & -I_{x y} \dot{p}_{b}-I_{y z} \dot{r}_{b}-I_{x y} q_{b} r_{b}+I_{y z} p_{b} q_{b}-m \Delta x_{b}\left(q_{b} u_{b}-\dot{w}_{b}-p_{b} v_{b}\right) \\
& -m \Delta z_{b}\left(\dot{u}_{b}-r_{b} v_{b}+q_{b} w_{b}\right)-m g\left(\sin \theta \Delta z_{b}+\cos \theta \cos \phi \Delta x_{b}\right)-\Delta z_{b} T \\
\Delta f_{4}= & -\left(p_{b} q_{b}+\dot{r}_{b}\right) \Delta x_{b}+\left(p_{b}^{2}+r_{b}^{2}\right) \Delta y_{b}-\left(q_{b} r_{b}-\dot{p}_{b}\right) \Delta z_{b} \\
\Delta f_{5}= & -I_{y z} \dot{q}_{b}+I_{x y}\left(p_{b}^{2}-q_{b}^{2}\right)-I_{y z} p_{b} r_{b}-m \Delta x_{b}\left(\dot{v}_{b}-p_{b} w_{b}+r_{b} u_{b}\right) \\
& -m \Delta y_{b}\left(r_{b} v_{b}-\dot{u}_{b}-q_{b} w_{b}\right)+m g \cos \theta \sin \phi \Delta x_{b}+m g \sin \theta \Delta y_{b}+T \Delta y_{b} \\
\Delta f_{6}= & -I_{x y} \dot{q}_{b}+I_{x y} p_{b} r_{b}-I_{y z}\left(q_{b}^{2}-r_{b}^{2}\right)-m \Delta y_{b}\left(\dot{w}_{b}-q_{b} u_{b}+p_{b} v_{b}\right) \\
& -m \Delta z_{b}\left(-\dot{v}_{b}-r_{b} u_{b}+p_{b} w_{b}\right)+m g \cos \theta \cos \phi \Delta y_{b}-m g \cos \theta \sin \phi \Delta z_{b} .
\end{aligned}
$$

Uncertain actuator failures. The actuators of the aircraft system may undergo some uncertain displacements when they are failed at some unknown time instants. 
The failed actuator control input can be modeled as

$$
u(t)=\left(I_{m}-\sigma\right) v(t)+\sigma \bar{u}(t),
$$

where $v(t)$ is the commanded control input signal, $\bar{u}(t)$ is the unknown failed actuator input signal, and elements of the failure index $\sigma=\operatorname{diag}\left\{\sigma_{1}, \sigma_{2}, \ldots, \sigma_{m}\right\}$ are $\sigma_{i}=1$ if the $i$ th actuator fails or $\sigma_{i}=0$ otherwise, for $i=1,2, \ldots, m$. It is worth noting that redundant actuators are widely employed in the aircraft flight control system, e.g., the GTM has a group of two rudder segments, a group of four elevator segments, etc.. This actuation redundancy provides us a potential tool to compensate the failed actuators by the remaining redundant healthy actuators.

Uncertain sensor failures. The sensor uncertainty model can be expressed as

$$
z(t)=k_{s} \varphi(t)+\sum_{i=1}^{q} b_{i} f_{i}(t)
$$

where $z(t)$ is the sensor measurement, $\varphi(t)$ is the actual signal, which is the state signal $x(t)$ or the output signal $y(t), k_{s}>0$ and $b_{i}, i=1,2, \ldots, q$, are some unknown constant sensor uncertainty parameters, and $f_{i}(t), i=1,2, \ldots, q$, are known bounded signals. The sensor model (2.1.17) can represent a class of practical sensor uncertainties such as sensor gain variations and measurement errors. If the individual sensor measurement cannot be modeled as (2.1.17), we can use a set of redundant sensors to measure the same signal $\varphi(t)$ and take the weighted sum of the sensors' output signals $z_{i}(t)$ as the signal measurement $z(t): z(t)=\sum_{i=1}^{m} \alpha_{i} z_{i}(t)$, such that $\sum_{i=1}^{m} \alpha_{i}=1$. With such a redundant sensor structure, the signal measurement $z(t)$ can still be modeled as (2.1.17): when there are no failures for all the sensors, the measurement $z(t)=\varphi(t)$; when uncertain sensor failures occur, e.g., the $i_{1}$ th, $i_{2}$ th, $\ldots, i_{p}$ th sensors are failed, the measurement is $z(t)=\alpha_{s} \varphi(t)+d_{s}(t)$, where $\alpha_{s}=\sum_{i \neq i_{1}, i_{2}, \ldots, i_{p}} \alpha_{i}$ is unknown and $d_{s}(t)=\sum_{i=1}^{m} \beta_{i} \bar{z}_{i}(t)$ with some of $\beta_{i}$ being zero (for the unfailed sensors) 
while others being $\alpha_{i}$, and $\bar{z}_{i}(t)$ being the measurement of each sensor.

Uncertain actuator nonlinearity models. When the actuators have nonlinearities, the system input vector signal $u(t)=\left[u_{1}(t), u_{2}(t), \ldots, u_{m}(t)\right]^{T}$ can be expressed as

$$
u(t)=N(v(t))
$$

where $N(\cdot)=\left[N_{1}(\cdot), N_{2}(\cdot)^{T}, \ldots, N_{m}(\cdot)\right]^{T}$ represents the actuator nonlinearities for some nonlinear functions $N_{i}(\cdot)$ such as deadzone, back-lash, or hysteresis. Consider the cases when $N_{i}(\cdot)$ can be parameterized as

$$
u_{i}(t)=N_{i}\left(v_{i}(t)\right)=-\theta_{N i}^{* T} \omega_{N i}^{*}(t)+a_{i}^{*}(t)
$$

for some unknown parameter vectors $\theta_{N i}^{*} \in R^{n_{i}}, n_{i} \geq 1, i=1, \ldots, M$, and some unknown regressor vector signals $\omega_{N i}^{*}(t) \in R^{n_{i}}$ and scalar signals $a_{i}^{*}(t)$. Such a parametrization has been established for $N_{i}(\cdot)$ being a dead-zone, backlash, hysteresis, or other characteristic [83], [84]. To cancel the effects of such actuator nonlinearities, we use a multivariable nonlinearity inverse model

$$
v(t)=\widehat{N I}\left(u_{d}(t)\right)
$$

where $u_{d}(t)=\left[u_{d 1}(t), \ldots, u_{d M}(t)\right]^{T}$ is a design vector signal from a feedback control law, that is,

$$
v_{i}(t)=\widehat{N I}_{i}\left(u_{d i}(t)\right), i=1, \ldots, M
$$

Then, each $\widehat{N I}_{i}(\cdot)$ can be parametrized as

$$
u_{d i}(t)=-\theta_{N i}^{T}(t) \omega_{N i}(t)+a_{i}(t), i=1, \ldots, M
$$

where $\theta_{N i} \in R^{n_{i}}$ is an estimate of $\theta_{N i}^{*}$ and $\omega_{N i}(t) \in R^{n_{i}}$ and $a_{i}(t)$ are some known signals, as in the case of an inverse for a dead-zone, backlash, or hysteresis [83], [84]. 
The uncertainties in $N_{i}(\cdot)$ cause a control error

$$
u_{i}(t)-u_{d i}(t)=\tilde{\theta}_{N i}^{T}(t) \omega_{N i}(t)+d_{N i}(t), i=1, \ldots, M
$$

where $\tilde{\theta}_{N i}=\theta_{N i}-\theta_{N i}^{*}$ and the unparameterized error is

$$
d_{N i}(t)=\theta_{N i}^{* T}\left(\omega_{N i}(t)-\omega_{N i}^{*}(t)\right)+a_{i}^{*}(t)-a_{i}(t)
$$

which should satisfy that conditions that $d_{N i}(t)$ is bounded, $t \geq 0$, and $d_{N i}(t)=0$, $t \geq t_{0}$, if $\theta_{N i}(t)=\theta_{N i}^{*}, t \geq t_{0}$, and $\widehat{N I}_{i}(\cdot)$ is correctly initialized: $d_{N i}\left(t_{0}\right)=0$. In vector form, the control error is

$$
u(t)-u_{d}(t)=\tilde{\Theta}_{N}^{T}(t) \omega_{N}(t)+d_{N}(t)
$$

where $\omega_{N}(t)=\left[\omega_{N 1}^{T}(t), \ldots, \omega_{N M}^{T}(t)\right]^{T}$ and

$$
\tilde{\Theta}_{N}^{T}(t)=\operatorname{diag}\left\{\tilde{\theta}_{N 1}^{T}(t), \tilde{\theta}_{N 2}^{T}(t), \ldots, \tilde{\theta}_{N M}^{T}(t)\right\} .
$$

The benchmark aircraft model-the NASA GTM. The proposed adaptive compensation and detection schemes will be verified and evaluated by the NASA GTM, which is shown in Fig. 2.1. The GTM is a 5.5\% dynamically scaled twinturbine powered test aircraft. Since the subscale GTM test results can be applied for the full-scale aircraft design, it is used to test flight research control designs in adverse conditions such as upsets, damage and failures [64]. In this research, the proposed designs will be applied to the Matlab/Simulink model of GTM. The Simulink model developed by the NASA contains engine dynamics, actuator dynamics, sensor dynamics, etc, which is a high-fidelity representative of the general nonlinear aircraft flight dynamics (2.1.1)-(2.1.9). Moreover, the GTM Simulink model provides some damage scenarios such as rudder off, vertical tail off, left outboard flap off, left wing-tip off, left elevator off, and left stabilizer off, and the actuator failures and the sensor failures can also be simulated. Hence, simulation offers a realistic representation of the 
aircraft under hazardous conditions and simulation results would provide a credible assessment of our proposed design.

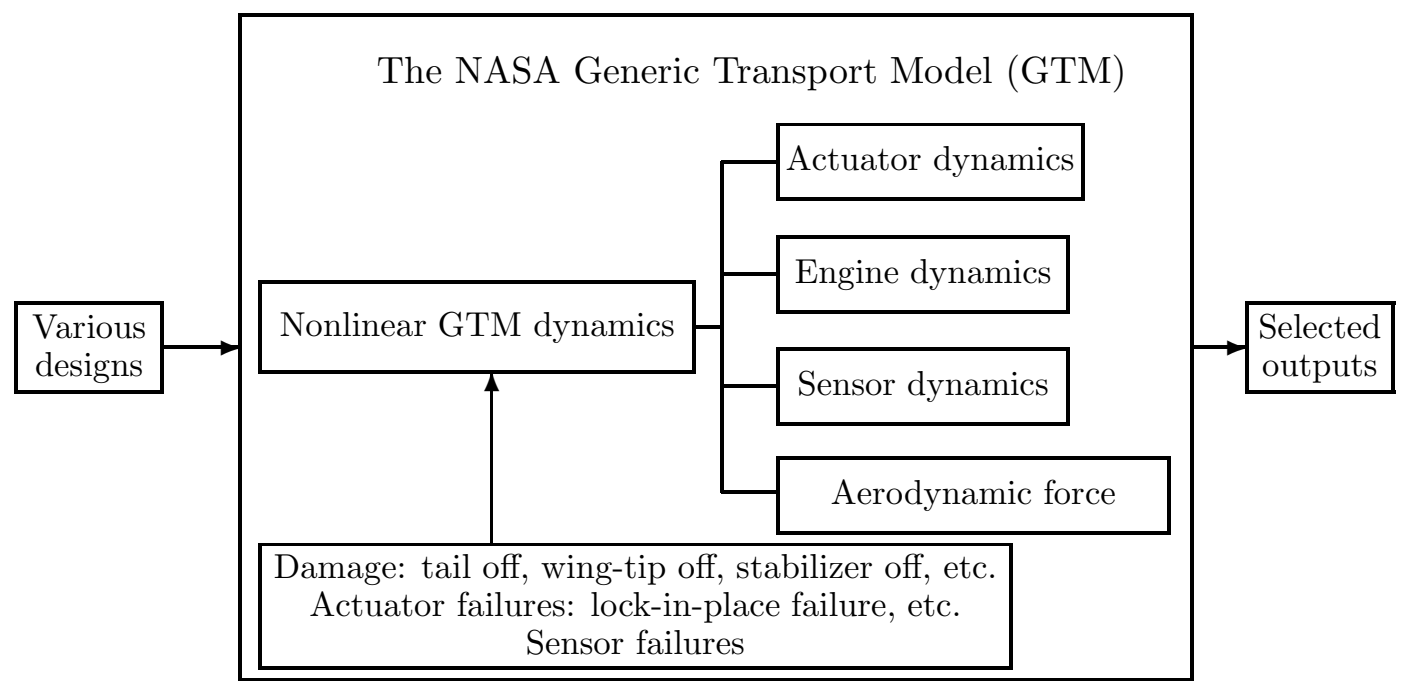

Figure 2.1: A benchmark aircraft model: the NASA GTM.

\subsection{Linearization-Based Design}

To handle the complicated nonlinear dynamics with uncertainties, a linearizationbased control design will be applied to the nonlinear aircraft flight system (2.1.10). Since there are uncertainties of the aircraft system with damage, the equilibrium point is not available. Therefore, an arbitrarily chosen operating point $\left(x_{0}, u_{0}\right)$, which may not be an equilibrium point, is used to linearize the nonlinear system (2.1.10) with damage and actuator failures:

$$
\Delta \dot{x}=A \Delta x+B \Delta u+f_{0}, \quad \Delta y=C \Delta x, \quad \Delta u=\left(I_{m}-\sigma\right) \Delta v+\sigma \Delta \bar{u},
$$

where $\Delta x=x-x_{0}, \Delta y=y-C x_{0}, \Delta u=u-u_{0}, \Delta v=v-u_{0}$ and $\Delta \bar{u}=\bar{u}-u_{0}$ are perturbation signals, and $A=\partial f /\left.\partial x\right|_{\left(x_{0}, u_{0}\right)}, B=\partial f /\left.\partial u\right|_{\left(x_{0}, u_{0}\right)}, f_{0}=f\left(x_{0}, u_{0}\right)$ are unknown piecewise constant parameters and dynamics offset due to different damage 
conditions, and the failure index $\sigma$ is also an uncertain piecewise constant function due to different failure patterns. Since the linearized model (2.2.1) is an approximation of the nonlinear aircraft system around a small neighborhood of the chosen operating point $\left(x_{0}, u_{0}\right)$, we will develop fault-tolerant control and fault detection schemes based on the linear model (2.2.1) with large system uncertainties. Figure 2.2 shows the linearization-based adaptive control and detection for the nonlinear aircraft system.

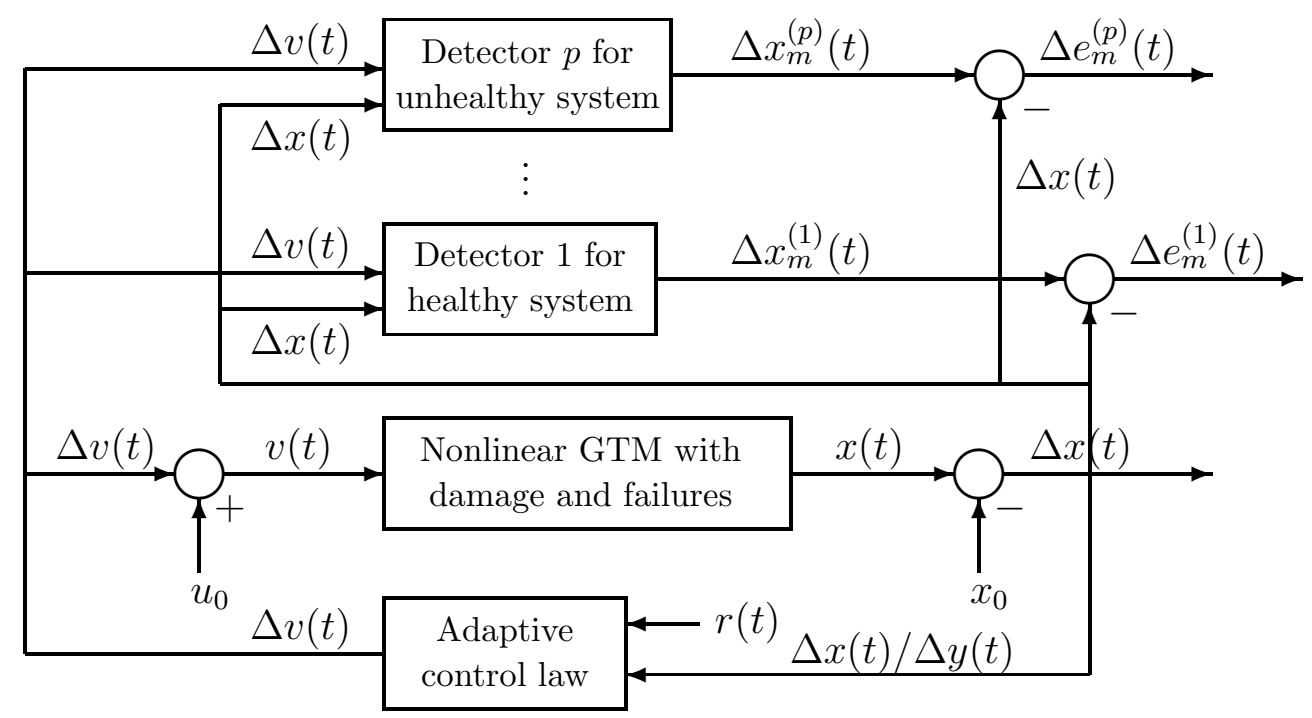

Figure 2.2: Linearization-based fault-tolerant control and fault detection.

\subsection{Linear System Preliminaries}

To proceed the multivariable model reference adaptive control (MRAC) design, some preliminaries are given as follows.

\subsubsection{Infinite Zero Structure}

For the MRAC of multi-input and multi-output (MIMO) systems, the system infinite zero structure characterized by its interactor matrix and high frequency gain matrix plays an important role. 
Lemma 2.3.1. [84], [82] For any $M \times M$ strictly proper and full rank rational matrix $G(s)$, there exists a lower triangular polynomial matrix $\xi_{m}(s)$, defined as the modified left interactor matrix of $G(s)$, of the form

$$
\xi_{m}(s)=\left[\begin{array}{ccccc}
d_{1}(s) & 0 & \cdots & \cdots & 0 \\
h_{21}^{m}(s) & d_{2}(s) & 0 & \cdots & 0 \\
\vdots & \vdots & \vdots & \vdots & \vdots \\
h_{M 1}^{m}(s) & \cdots & \cdots & h_{M M-1}^{m}(s) & d_{M}(s)
\end{array}\right]
$$

where $h_{i j}^{m}(s), j=1, \ldots, M-1, i=2, \ldots, M$, are polynomials, and $d_{i}(s), i=$ $1, \ldots, M$, are any chosen monic stable polynomials of degrees $l_{i}>0$, such that the associated high frequency gain matrix

$$
K_{p}=\lim _{s \rightarrow \infty} \xi_{m}(s) G(s)
$$

is finite and nonsingular.

From Lemma 2.3.1, we can see that the interactor matrix $\xi_{m}(s)$ and its high frequency gain matrix $K_{p}$ capture essential characteristics of the zero structure of the system transfer matrix $G(s)$ at infinity $(s=\infty)$. For our multivariable MRAC design, we will choose the reference system as $W_{m}(s)=\xi_{m}^{-1}(s)$. Based on the knowledge of the infinite zero structure, i.e., the interactor matrix and the high frequency gain matrix, output matching control schemes (to be shown next), which will be used as plant-model matching conditions for the adaptive control designs, can be established to achieve output tracking of a chosen reference signal $y_{m}(t)=W_{m}(s)[r](t)$.

\subsubsection{Gain Matrix Decomposition}

For multivariable MRAC control design, certain knowledge of the system high frequency gain matrix $K_{p}$ is important for adaptive law construction. Under the condition that all leading principal minors of $K_{p}$ are nonzero, several decompositions of $K_{p}$ exist and can be used to reduce the knowledge of $K_{p}$. 
LDU decomposition of $K_{p}[23]$. Let $\Delta_{i}, i=1,2, \ldots, M$, be the leading principal minors of $K_{p} \in R^{M \times M}$, and assume that $\Delta_{i} \neq 0, i=1,2, \ldots, M$. Then, $K_{p}$ has a unique LDU decomposition:

$$
K_{p}=L D U
$$

for some $M \times M$ unit (that is, with all diagonal elements being 1) lower triangular matrix $L$ and unity upper triangular matrix $U$, and

$$
D=\operatorname{diag}\left\{d_{1}^{*}, d_{2}^{*}, \ldots, d_{M}^{*}\right\}=\operatorname{diag}\left\{\Delta_{1}, \frac{\Delta_{2}}{\Delta_{1}}, \ldots, \frac{\Delta_{M}}{\Delta_{M-1}}\right\}
$$

For the LDU decomposition of an unknown $K_{p}$, we also assume that the signs of $\Delta_{i}, i=1,2, \ldots, M$, are known. Then, in view of (2.3.4), $\operatorname{sign}\left[d_{i}^{*}\right]$, the sign of $d_{i}^{*}$, is also known, $i=1,2, \ldots, M$, and it will be used in constructing stable adaptive parameter estimation schemes.

LDS and SDU decompositions of $K_{p}$. Similar to its LDU decomposition, the gain matrix $K_{p} \in R^{M \times M}$ with all its leading principal minors $\Delta_{i} \neq 0, i=1,2, \ldots, M$, also has a non-unique LDS decomposition:

$$
K_{p}=L_{s} D_{s} S
$$

where $S \in R^{M \times M}$ with $S=S^{T}>0, L_{s}$ is an $M \times M$ unity lower triangular matrix, and

$$
\begin{aligned}
D_{s} & =\operatorname{diag}\left\{s_{1}^{*}, s_{2}^{*}, \ldots, s_{M}^{*}\right\} \\
& =\operatorname{diag}\left\{\operatorname{sign}\left[\Delta_{1}\right] \gamma_{1}, \operatorname{sign}\left[\frac{\Delta_{2}}{\Delta_{1}}\right] \gamma_{2}, \ldots, \operatorname{sign}\left[\frac{\Delta_{M}}{\Delta_{M-1}}\right] \gamma_{M}\right\}
\end{aligned}
$$

such that $\gamma_{i}>0, i=1, \ldots, M$, may be arbitrarily chosen.

This LDS decomposition of $K_{p}$ follows from the LDU decomposition of $K_{p}$, with $L_{s}=L D_{s} U^{-T} D_{s}^{-1}$ and $S=U^{T} D_{s}^{-1} D U$. The choice of $D_{s}$ is not unique, which provides certain flexibility in designing stable adaptive laws. 
A similar SDU decomposition of $K_{p}$ is: $K_{p}=S D_{s} U_{s}$, for $S=S^{T}=L D D_{s}^{-1} L^{T}>0$ and $U_{s}=D_{s}^{-1} L^{-T} D_{s} U$ unity upper triangular.

Based on its LDU, LDS or SDU decomposition, the uncertainties of $K_{p}$ can be further dealt with using stable estimation schemes to estimate the unknown parameters in $L$ and $U$, while the knowledge of $S$ and $D$ either is not needed (because $\left.S=S^{T}>0\right)$ or can be specified in terms of $\operatorname{sign}\left[\Delta_{i}\right]$.

\subsection{Output Plant-Model Matching Control}

An important control objective of our designs in this research is to achieve system output signal tracking of a chosen reference signal, while the system dynamics have parametric and structural uncertainties. Here, we will present two nominal control designs with state feedback and output feedback respectively for known system dynamics to achieve exact plant-model matching, where the plant-model matching conditions will be used for developing multivariable MRAC schemes in the following chapters to accommodate the system uncertainties.

Consider a general multi-input and multi-output linear time-invariant system

$$
\dot{x}(t)=A x(t)+B u(t), y(t)=C x(t)
$$

where $A \in R^{n \times n}, B \in R^{n \times M}$, and $C \in R^{M \times n},(A, B)$ is stabilizable and $(A, C)$ is detectable, the transfer matrix $G(s)=C(s I-A)^{-1} B$ has full rank, and all zeros of $G(s)$ are stable.

\subsubsection{State Feedback Control Design}

The objective is to design a state feedback control law

$$
u(t)=K_{1}^{* T} x(t)+K_{2}^{*} r(t)
$$


for some constant matrices $K_{1}^{*} \in R^{n \times M}$ and $K_{2}^{*} \in R^{M \times M}$, to make the closed-loop system transfer matrix

$$
C\left(s I-A-B K_{1}^{* T}\right)^{-1} B K_{2}^{*}=W_{m}(s), W_{m}(s)=\xi_{m}^{-1}(s),
$$

with $\xi_{m}(s)$ being the modified interactor matrix of $G(s)$.

Design with a diagonal $\xi_{m}(s)$. We first consider the case, where the modified interactor matrix $\xi_{m}(s)$ is of diagonal form

$$
\xi_{m}(s)=\operatorname{diag}\left\{d_{1}(s), d_{2}(s), \ldots, d_{M}(s)\right\},
$$

where $d_{i}(s), i=1,2, \ldots, M$, are some monic stable polynomials of degrees $l_{i}>0$, such that $\lim _{s \rightarrow \infty} \xi_{m}(s) G(s)=K_{p}$ is finite and nonsingular. Furthermore, from the expression of $G(s)$ :

$$
G(s)=C(s I-A)^{-1} B=C\left(I \frac{1}{s}+A \frac{1}{s^{2}}+A^{2} \frac{1}{s^{3}}+A^{3} \frac{1}{s^{4}}+\cdots\right) B,
$$

it follows that the associated high frequency gain matrix can be calculated as

$$
K_{p}=\left[\begin{array}{c}
c_{1} A^{l_{1}-1} B \\
c_{2} A^{l_{2}-1} B \\
\vdots \\
c_{M} A^{l_{M}-1} B
\end{array}\right],
$$

which is nonsingular, with $c_{i}, i=1,2, \ldots, M$, denoting the $i$ th row of $C$.

From the system equations $\dot{x}=A x+B u, y_{i}=c_{i} x$, for $y=\left[y_{1}, y_{2}, \ldots, y_{M}\right]^{T}$, denoting the $j$ th order time-derivative of $y_{i}(t)$ as $y_{i}^{(j)}(t)$, we have

$$
y_{i}^{(j)}(t)= \begin{cases}c_{i} A^{j} x(t) & \text { for } j=0,1, \ldots, l_{i}-1 \\ c_{i} A^{j} x(t)+c_{i} A^{j-1} B u(t) & \text { for } j=l_{i} .\end{cases}
$$

Considering the last equations

$$
y_{i}^{\left(l_{i}\right)}(t)=c_{i} A^{l_{i}} x(t)+c_{i} A^{l_{i}-1} B u(t), i=1,2, \ldots, M
$$


we first choose a control law

$$
u(t)=K_{p}^{-1} v(t), v=\left[v_{1}, v_{2}, \ldots, v_{M}\right]^{T}
$$

to make (2.4.8) to the individual equations controlled by $v_{i}(t)$ :

$$
y_{i}^{\left(l_{i}\right)}(t)=c_{i} A^{l_{i}} x(t)+v_{i}(t), i=1,2, \ldots, M
$$

Then, based on a choice of the modified interactor matrix

$$
\xi_{m}(s)=\operatorname{diag}\left\{d_{1}(s), d_{2}(s), \ldots, d_{M}(s)\right\}
$$

where $d_{i}(s)=s^{l_{i}}+a_{i 1} s^{l_{i}-1}+\cdots+a_{i l_{i}-1} s+a_{i l_{i}}$ is stable, $i=1,2, \ldots, M$, we choose the feedback control law

$$
\begin{aligned}
v_{i}(t)= & -c_{i} A^{l_{i}} x(t)-a_{i 1} y_{i}^{\left(l_{i}-1\right)}(t)-\cdots-a_{i l_{i}-1} y_{i}^{(1)}(t)-a_{i l_{i}} y_{i}(t)+r_{i}(t) \\
= & -c_{i} A^{l_{i}} x(t)-a_{i 1} c_{i} A^{l_{i}-1} x(t)-\cdots-a_{i l_{i}-1} c_{i} A x(t) \\
& -a_{i l_{i}} c_{i} x(t)+r_{i}(t)
\end{aligned}
$$

for some reference input signals $r_{i}(t), i=1,2, \ldots, M$, to make (2.4.10) into the decoupled equations:

$$
y_{i}^{\left(l_{i}\right)}(t)+a_{i 1} y_{i}^{\left(l_{i}-1\right)}(t)+\cdots+a_{i l_{i}-1} y_{i}^{(1)}(t)+a_{i l_{i}} y_{i}(t)=r_{i}(t)
$$

for $i=1,2, \ldots, M$. The control law (2.4.12) can be written as

$$
v(t)=K_{0} x(t)+r(t), r=\left[r_{1}, r_{2}, \ldots, r_{M}\right]^{T},
$$

where the $i$ th row of $K_{0}$ is

$$
k_{0 i}^{T}=-c_{i} A^{l_{i}}-a_{i 1} c_{i} A^{l_{i}-1}-\cdots-a_{i l_{i}-1} c_{i} A-a_{i l_{i}} c_{i},
$$

for $i=1,2, \ldots, M$. 
Hence, it follows from (2.4.9) and (2.4.14) that

$$
\begin{aligned}
u(t) & =K_{p}^{-1} K_{0} x(t)+K_{p}^{-1} r(t) \\
& \triangleq K_{1}^{* T} x(t)+K_{2}^{*} r(t),
\end{aligned}
$$

where

$$
K_{1}^{* T}=K_{p}^{-1} K_{0} \in R^{M \times n}, K_{2}^{*}=K_{p}^{-1} \in R^{M \times M}
$$

Therefore, when applying the state feedback control law $u(t)$ in $(2.4 .16)$ with $K_{1}^{*}$ and $K_{2}^{*}$ in $(2.4 .17)$ to the system $(2.4 .1)$, we have the closed-loop system as

$$
y(t)=C\left(s I-A-B K_{1}^{* T}\right)^{-1} B K_{2}^{*}[r](t)=\xi_{m}^{-1}(s)[r](t),
$$

where $\xi_{m}(s)$ is of the diagonal form (2.4.4). That is the plant-model matching condition (2.4.3) holds for the diagonal $\xi_{m}(s)$.

Design with a non-diagonal $\xi_{m}(s)$. For $G(s)=C(s I-A)^{-1} B$ with a nondiagonal interactor matrix $\xi(s)$, there also exist $K_{1}^{*} \in R^{n \times M}$ and $K_{2}^{*} \in R^{M \times M}$ such that $C\left(s I-A-B K_{1}^{* T}\right)^{-1} B K_{2}^{*}=\xi_{m}^{-1}(s)$ [59], [62], that is, for the state feedback control law $u(t)=K_{1}^{* T} x(t)+K_{2}^{*} r(t)$, the closed-loop system matches the reference system $y(t)=\xi_{m}^{-1}(s)[r](t)$.

Here, as an illustrative example: we consider a direct calculation of $K_{1}^{*}$ and $K_{2}^{*}$ for a 2-input and 2-output system $G(s)$ with a non-diagonal modified interactor matrix $\xi_{m}(s)$. Let $G_{i}(s)$ be the $i$ th row of $G(s)$ and $\mu_{i}$ be the unique integers such that

$$
\lim _{s \rightarrow \infty} s^{\mu_{i}} G_{i}(s)=\tau_{i}
$$

is finite and nonzero, for $i=1,2$, and set $\xi_{1}(s)$ and $\xi_{2}^{0}(s)$ as

$$
\xi_{1}(s)=\left[s^{\mu_{1}}, 0\right], \quad \xi_{2}^{0}(s)=\left[0, s^{\mu_{2}}\right]
$$


Since the interactor matrix is non-diagonal, $\tau_{2}$ is linearly dependent of $\tau_{1}$, such that $\tau_{2}=\alpha_{21}^{1} \tau_{1}$ for some $\alpha_{21}^{1} \neq 0$. We let

$$
\xi_{2}^{1}(s)=s^{\mu_{2}^{1}}\left(\xi_{2}^{0}(s)-\alpha_{21}^{1} \xi_{1}(s)\right)=s^{\mu_{2}^{1}}\left[-\alpha_{21}^{1} s^{\mu_{1}}, s^{\mu_{2}}\right]
$$

for some unique integer $\mu_{2}^{1}>0$ such that

$$
\lim _{s \rightarrow \infty} \xi_{2}^{1}(s) G(s)=\tau_{2}^{1}
$$

is finite and nonzero. If $\tau_{2}^{1}$ is linearly independent of $\tau_{1}$, then we set $\xi_{2}(s)=\xi_{2}^{1}(s)$. If $\tau_{2}^{1}$ is linearly dependent of $\tau_{1}$, such that $\tau_{2}^{1}=\alpha_{21}^{2} \tau_{1}$ for some constant $\alpha_{21}^{2} \neq 0$, then we let

$$
\xi_{2}^{2}(s)=s^{\mu_{2}^{2}}\left(\xi_{2}^{1}(s)-\alpha_{21}^{2} \xi_{1}(s)\right)=\left[-\alpha_{21}^{1} s^{\mu_{1}+\mu_{2}^{1}+\mu_{2}^{2}}-\alpha_{21}^{2} s^{\mu_{1}+\mu_{2}^{2}}, s^{\mu_{2}+\mu_{2}^{1}+\mu_{2}^{2}}\right]
$$

for some unique integer $\mu_{2}^{2}>0$ such that

$$
\lim _{s \rightarrow \infty} \xi_{2}^{2}(s) G(s)=\tau_{2}^{2}
$$

is finite and nonzero. If $\tau_{2}^{2}$ is linearly dependent of $\tau_{1}$, one can repeat the above procedure until getting a $\xi_{2}^{k}(s)$ with a $\mu_{2}^{k}>0$ for some $k>2$ such that $\lim _{s \rightarrow \infty} \xi_{2}^{k}(s) G(s)=$ $\tau_{2}^{k}$ is linearly independent of $\tau_{1}$. Then we can set $\xi_{2}(s)=\xi_{2}^{k}(s)$. The formation of the linearly independent $\xi_{1}(s)$ and $\xi_{1}(s)$ is based on a construction procedure in [89]. For this illustrative example, we assume that $\xi_{1}(s)$ and $\xi_{2}^{1}(s)$ are linearly independent, such that there exists an interactor matrix

$$
\xi(s)=\left[\begin{array}{l}
\xi_{1}(s) \\
\xi_{2}^{1}(s)
\end{array}\right]=\left[\begin{array}{cc}
s^{\mu_{1}} & 0 \\
-\alpha_{21}^{1} s^{\mu_{1}+\mu_{2}^{1}} & s^{\mu_{2}+\mu_{2}^{1}}
\end{array}\right]
$$

to make

$$
\lim _{s \rightarrow \infty} \xi(s) G(s)=K_{p}=\left[\begin{array}{c}
\tau_{1} \\
\tau_{2}^{1}
\end{array}\right],
$$

which has full rank. Then, we will show the calculation of $K_{1}^{*}, K_{2}^{*}$, and the modified left interactor matrix $\xi_{m}(s)$. 
Without loss of generality, we assume $\mu_{1} \geq \mu_{2}$. With $c_{i}$ being the $i$ th row of $C$, the definition of $\mu_{i}$ implies that

$$
\tau_{i}=c_{i} A^{\mu_{i}-1} B \neq 0_{1 \times M}, c_{i} A^{j} B=0_{1 \times M}, j=0,1, \ldots, \mu_{i}-2, i=1,2 .
$$

In terms of the output signals $y_{1}(t)$ and $y_{2}(t)$, we have

$$
y_{i}^{(j)}(t)= \begin{cases}c_{i} A^{j} x(t) & \text { for } j=0,1, \ldots, \mu_{i}-1 \\ c_{i} A^{j} x(t)+c_{i} A^{j-1} B u(t) & \text { for } j=\mu_{i} .\end{cases}
$$

Different from the case with a diagonal $\xi(s)$ where both $y_{1}(t)$ and $y_{2}(t)$ equations are used for feedback control design, we will only use those $y_{1}(t)$ equations, plus a combination of the $y_{1}(t)$ equations and $y_{2}(t)$ equations, for the case with a nondiagonal $\xi(s)$. The definition of $\mu_{2}^{1}$ implies that

$$
\begin{gathered}
\tau_{2}^{1}=-\alpha_{21}^{1} c_{1} A^{\mu_{1}-1+\mu_{2}^{1}} B+c_{2} A^{\mu_{2}-1+\mu_{2}^{1}} B \\
-\alpha_{21}^{1} c_{1} A^{\mu_{1}-1+k} B+c_{2} A^{\mu_{2}-1+k} B=0_{1 \times M}, k=0,1, \ldots, \mu_{2}^{1}-1 .
\end{gathered}
$$

In view of these relationships, we introduce the new output variable

$$
\begin{aligned}
z_{2}(t)= & -\alpha_{21}^{1}\left(y_{1}^{\left(\mu_{1}\right)}(t)+\beta_{1} y_{1}^{\left(\mu_{1}-1\right)}(t)+\beta_{2} y_{1}^{\left(\mu_{1}-2\right)}(t)+\cdots+\beta_{\mu_{2}} y_{1}^{\left(\mu_{1}-\mu_{2}\right)}(t)\right) \\
& +y_{2}^{\left(\mu_{2}\right)}(t)+\beta_{1} y_{2}^{\left(\mu_{2}-1\right)}(t)+\beta_{2} y_{2}^{\left(\mu_{2}-2\right)}(t)+\cdots \beta_{\mu_{2}} y_{2}(t)
\end{aligned}
$$

which can be also expressed as

$$
z_{2}(t)=-\alpha_{21}^{1} \bar{h}_{2}(s)\left[y_{1}\right](t)+\bar{d}_{21}(s)\left[y_{2}\right](t),
$$

where the polynomials $\bar{h}_{2}(s)$ and $\bar{d}_{21}(s)$ are

$$
\begin{aligned}
& \bar{h}_{2}(s)=s^{\mu_{1}}+\beta_{1} s^{\mu_{1}-1}+\beta_{2} s^{\mu_{1}-2}+\cdots+\beta_{\mu_{2}-1} s^{\mu_{1}-\mu_{2}+1}+\beta_{\mu_{2}} s^{\mu_{1}-\mu_{2}} \\
& \bar{d}_{21}(s)=s^{\mu_{2}}+\beta_{1} s^{\mu_{2}-1}+\beta_{2} s^{\mu_{2}-2}+\cdots+\beta_{\mu_{2}-1} s+\beta_{\mu_{2}}
\end{aligned}
$$


with some chosen $\beta_{i}, i=1,2, \ldots, \mu_{2}$, to make $\bar{d}_{21}(s)$ stable. Then, from the expressions of the derivatives of the output signals $y_{1}(t)$ and $y_{2}(t)$ as given in (2.4.28), we have $z_{2}(t)$ in $(2.4 .31)$ as

$$
\begin{aligned}
z_{2}(t)= & -\alpha_{21}^{1}\left(c_{1} A^{\mu_{1}} x(t)+c_{1} A^{\mu_{1}-1} B u(t)\right)+\left(c_{2} A^{\mu_{2}} x(t)+c_{2} A^{\mu_{2}-1} B u(t)\right) \\
& -\alpha_{21}^{1}\left(\beta_{1} c_{1} A^{\mu_{1}-1}+\beta_{2} c_{1} A^{\mu_{1}-2}+\cdots+\beta_{\mu_{2}} c_{1} A^{\mu_{1}-\mu_{2}}\right) x(t) \\
& +\left(\beta_{1} c_{2} A^{\mu_{2}-1}+\beta_{2} c_{2} A^{\mu_{2}-2}+\cdots+\beta_{\mu_{2}} c_{2}\right) x(t) .
\end{aligned}
$$

In view of (2.4.30) with $k=0$, i.e., $-\alpha_{21}^{1} c_{1} A^{\mu_{1}-1} B+c_{2} A^{\mu_{2}-1} B=0$, it follows

$$
\begin{aligned}
z_{2}(t)= & \left(-\alpha_{21}^{1}\left(c_{1} A^{\mu_{1}}+\beta_{1} c_{1} A^{\mu_{1}-1}+\beta_{2} c_{1} A^{\mu_{1}-2}+\cdots+\beta_{\mu_{2}} c_{1} A^{\mu_{1}-\mu_{2}}\right)\right. \\
& \left.+c_{2} A^{\mu_{2}}+\beta_{1} c_{2} A^{\mu_{2}-1}+\beta_{2} c_{2} A^{\mu_{2}-2}+\cdots+\beta_{\mu_{2}} c_{2}\right) x(t) .
\end{aligned}
$$

By taking derivative on both sides of (2.4.36) and from $\dot{x}=A x+B u$, we have

$$
\begin{aligned}
z_{2}^{(1)}(t)= & \left(-\alpha_{21}^{1}\left(c_{1} A^{\mu_{1}+1}+\beta_{1} c_{1} A^{\mu_{1}}+\beta_{2} c_{1} A^{\mu_{1}-1}+\cdots+\beta_{\mu_{2}} c_{1} A^{\mu_{1}-\mu_{2}+1}\right)\right. \\
& \left.+c_{2} A^{\mu_{2}+1}+\beta_{1} c_{2} A^{\mu_{2}}+\beta_{2} c_{2} A^{\mu_{2}-1}+\cdots+\beta_{\mu_{2}} c_{2} A\right) x(t) \\
+ & \left(-\alpha_{21}^{1}\left(c_{1} A^{\mu_{1}} B+\beta_{1} c_{1} A^{\mu_{1}-1} B+\beta_{2} c_{1} A^{\mu_{1}-2} B+\cdots+\beta_{\mu_{2}} c_{1} A^{\mu_{1}-\mu_{2}} B\right)\right. \\
& \left.+c_{2} A^{\mu_{2}} B+\beta_{1} c_{2} A^{\mu_{2}-1} B+\beta_{2} c_{2} A^{\mu_{2}-2} B+\cdots+\beta_{\mu_{2}} c_{2} B\right) u(t),
\end{aligned}
$$

in view of (2.4.30) with $k=0,1$, i.e.,

$$
-\alpha_{21}^{1} c_{1} A^{\mu_{1}-1} B+c_{2} A^{\mu_{2}-1} B=0, \quad-\alpha_{21}^{1} c_{1} A^{\mu_{1}} B+c_{2} A^{\mu_{2}} B=0,
$$

and (2.4.27) with $c_{i} A^{j} B=0$ for $i=1,2, j \leq \mu_{i}-2$, the coefficient of $u(t)$ is 0 , so that $z_{2}^{(1)}(t)$ is

$$
\begin{aligned}
z_{2}^{(1)}(t) & =\left(-\alpha_{21}^{1}\left(c_{1} A^{\mu_{1}+1}+\beta_{1} c_{1} A^{\mu_{1}}+\beta_{2} c_{1} A^{\mu_{1}-1}+\cdots+\beta_{\mu_{2}} c_{1} A^{\mu_{1}-\mu_{2}+1}\right)\right. \\
& \left.+c_{2} A^{\mu_{2}+1}+\beta_{1} c_{2} A^{\mu_{2}}+\beta_{2} c_{2} A^{\mu_{2}-1}+\cdots+\beta_{\mu_{2}} c_{2} A\right) x(t)
\end{aligned}
$$


Similarly, for the $j$ th order derivative of $z_{2}(t)$ with $j=2, \ldots, \mu_{2}^{1}-1$, we have

$$
\begin{aligned}
z_{2}^{(j)}(t) & =\left(-\alpha_{21}^{1}\left(c_{1} A^{\mu_{1}+j}+\beta_{1} c_{1} A^{\mu_{1}-1+j}+\beta_{2} c_{1} A^{\mu_{1}-2+j}+\cdots+\beta_{\mu_{2}} c_{1} A^{\mu_{1}-\mu_{2}+j}\right)\right. \\
& \left.+c_{2} A^{\mu_{2}+j}+\beta_{1} c_{2} A^{\mu_{2}-1+j}+\beta_{2} c_{2} A^{\mu_{2}-2+j}+\cdots+\beta_{\mu_{2}} c_{2} A^{j}\right) x(t) \\
& +\left(-\alpha_{21}^{1}\left(c_{1} A^{\mu_{1}-1+j} B+\beta_{1} c_{1} A^{\mu_{1}-2+j} B+\cdots+\beta_{\mu_{2}} c_{1} A^{\mu_{1}-\mu_{2}-1+j} B\right)\right. \\
& \left.+c_{2} A^{\mu_{2}-1+j} B+\beta_{1} c_{2} A^{\mu_{2}-2+j} B+\cdots+\beta_{\mu_{2}} c_{2} A^{-1+j} B\right) u(t)
\end{aligned}
$$

and from (2.4.27) and (2.4.30), the coefficient of $u(t)$ becomes 0 , that is

$$
\begin{aligned}
z_{2}^{(j)}(t) & =\left(-\alpha_{21}^{1}\left(c_{1} A^{\mu_{1}+j}+\beta_{1} c_{1} A^{\mu_{1}-1+j}+\beta_{2} c_{1} A^{\mu_{1}-2+j}+\cdots+\beta_{\mu_{2}} c_{1} A^{\mu_{1}-\mu_{2}+j}\right)\right. \\
& \left.+c_{2} A^{\mu_{2}+j}+\beta_{1} c_{2} A^{\mu_{2}-1+j}+\beta_{2} c_{2} A^{\mu_{2}-2+j}+\cdots+\beta_{\mu_{2}} c_{2} A^{j}\right) x(t) .
\end{aligned}
$$

Then, by taking derivative on both sides of (2.4.38) for $j=\mu_{2}^{1}-1$ and from $\dot{x}=$ $A x+B u$, we obtain the $\mu_{2}^{1}$ th order derivative of $z_{2}(t)$ :

$$
\begin{aligned}
z_{2}^{\left(u_{2}^{1}\right)}(t) & =\left(-\alpha_{21}^{1}\left(c_{1} A^{\mu_{1}+\mu_{2}^{1}}+\beta_{1} c_{1} A^{\mu_{1}-1+\mu_{2}^{1}}+\beta_{2} c_{1} A^{\mu_{1}-2+\mu_{2}^{1}}+\cdots+\beta_{\mu_{2}} c_{1} A^{\mu_{1}-\mu_{2}+\mu_{2}^{1}}\right)\right. \\
& \left.+c_{2} A^{\mu_{2}+\mu_{2}^{1}}+\beta_{1} c_{2} A^{\mu_{2}-1+\mu_{2}^{1}}+\beta_{2} c_{2} A^{\mu_{2}-2+\mu_{2}^{1}}+\cdots+\beta_{\mu_{2}} c_{2} A^{\mu_{2}^{1}}\right) x(t) \\
& +\left(-\alpha_{21}^{1}\left(c_{1} A^{\mu_{1}-1+\mu_{2}^{1}} B+\beta_{1} c_{1} A^{\mu_{1}-2+\mu_{2}^{1}} B+\cdots+\beta_{\mu_{2}} c_{1} A^{\mu_{1}-\mu_{2}-1+\mu_{2}^{1}} B\right)\right. \\
& \left.+c_{2} A^{\mu_{2}-1+\mu_{2}^{1}} B+\beta_{1} c_{2} A^{\mu_{2}-2+\mu_{2}^{1}} B+\cdots+\beta_{\mu_{2}} c_{2} A^{-1+\mu_{2}^{1}} B\right) u(t),
\end{aligned}
$$

from $(2.4 .27),(2.4 .29)$, and $(2.4 .30)$, the coefficient of $u(t)$ becomes

$$
\tau_{2}^{1}=-\alpha_{21}^{1} c_{1} A^{\mu_{1}-1+\mu_{2}^{1}} B+c_{2} A^{\mu_{2}-1+\mu_{2}^{1}} B
$$

it follows that

$$
\begin{aligned}
z_{2}^{\left(u_{2}^{1}\right)}(t) & =\left(-\alpha_{21}^{1}\left(c_{1} A^{\mu_{1}+\mu_{2}^{1}}+\beta_{1} c_{1} A^{\mu_{1}-1+\mu_{2}^{1}}+\beta_{2} c_{1} A^{\mu_{1}-2+\mu_{2}^{1}}+\cdots+\beta_{\mu_{2}} c_{1} A^{\mu_{1}-\mu_{2}+\mu_{2}^{1}}\right)\right. \\
& \left.+c_{2} A^{\mu_{2}+\mu_{2}^{1}}+\beta_{1} c_{2} A^{\mu_{2}-1+\mu_{2}^{1}}+\beta_{2} c_{2} A^{\mu_{2}-2+\mu_{2}^{1}}+\cdots+\beta_{\mu_{2}} c_{2} A^{\mu_{2}^{1}}\right) x+\tau_{2}^{1} u
\end{aligned}
$$

In summary, from (2.4.36), (2.4.37), (2.4.38), and (2.4.39), we have

$$
z_{2}^{(j)}(t)= \begin{cases}D_{j} x(t) & \text { for } j=0,1, \ldots, \mu_{2}^{1}-1 \\ D_{j} x(t)+\tau_{2}^{1} u(t) & \text { for } j=\mu_{2}^{1},\end{cases}
$$


where the matrices $D_{j}, j=0,1, \ldots, \mu_{2}^{1}$, are

$$
\begin{aligned}
D_{j}= & -\alpha_{21}^{1}\left(c_{1} A^{\mu_{1}+j}+\beta_{1} c_{1} A^{\mu_{1}-1+j}+\beta_{2} c_{1} A^{\mu_{1}-2+j}+\cdots+\beta_{\mu_{2}} c_{1} A^{\mu_{1}-\mu_{2}+j}\right) \\
& +c_{2} A^{\mu_{2}+j}+\beta_{1} c_{2} A^{\mu_{2}-1+j}+\beta_{2} c_{2} A^{\mu_{2}-2+j}+\cdots+\beta_{\mu_{2}-1} c_{2} A^{1+j}+\beta_{\mu_{2}} c_{2} A^{j} .
\end{aligned}
$$

We now derive the feedback control design. From the last equation of (2.4.28), for the first output signal $y_{1}(t)$, we have

$$
y_{1}^{\left(\mu_{1}\right)}(t)=c_{1} A^{\mu_{1}} x(t)+\tau_{1} u(t)
$$

Then, with the choice of a stable polynomial

$$
d_{1}(s)=s^{\mu_{1}}+a_{11} s^{\mu_{1}-1}+\cdots+a_{1 \mu_{1}-1} s+a_{1 \mu_{1}},
$$

we choose the feedback control law component

$$
\tau_{1} u(t)=-c_{1} A^{\mu_{1}} x(t)-a_{11} y_{1}^{\left(\mu_{1}-1\right)}(t)-\cdots-a_{1 \mu_{1}-1} y_{i}^{(1)}(t)-a_{1 \mu_{1}} y_{1}(t)+r_{1}(t),
$$

for some reference input signal $r_{1}(t)$, to make (2.4.42) into the decoupled equation:

$$
y_{1}^{\left(\mu_{1}\right)}(t)+a_{11} y_{1}^{\left(\mu_{1}-1\right)}(t)+\cdots+a_{1 \mu_{1}-1} y_{1}^{(1)}(t)+a_{1 \mu_{1}} y_{1}(t)=r_{1}(t)
$$

Moreover, from equations of (2.4.28), the control law (2.4.44) can be realized using the state feedback structure:

$$
\begin{aligned}
\tau_{1} u(t)= & -c_{1} A^{\mu_{1}} x(t)-a_{11} c_{1} A^{\mu_{1}-1} x(t)-\cdots \\
& -a_{1 \mu_{1}-1} c_{1} A x(t)-a_{1 \mu_{1}} c_{1} x(t)+r_{1}(t),
\end{aligned}
$$

From the last equation of (2.4.40), we have

$$
z_{2}^{\left(\mu_{2}^{1}\right)}(t)=D_{\mu_{2}^{1}} x(t)+\tau_{2}^{1} u(t)
$$

With the choice of a stable polynomial

$$
\bar{d}_{22}(s)=s^{\mu_{2}^{1}}+a_{21} s^{\mu_{2}^{1}-1}+\cdots+a_{2 \mu_{2}^{1}-1} s+a_{2 \mu_{2}^{1}},
$$


we choose the second feedback control law component

$$
\begin{aligned}
\tau_{2}^{1} u(t)= & -D_{\mu_{2}^{1}} x(t)-a_{21} z_{2}^{\left(\mu_{2}^{1}-1\right)}(t)-\cdots \\
& -a_{2 \mu_{2}^{1}-1} z_{2}^{(1)}(t)-a_{2 \mu_{2}^{1}} z_{2}(t)+r_{2}(t),
\end{aligned}
$$

for some reference input signal $r_{2}(t)$, to make (2.4.47) to a decoupled equation in terms of the new output variable $z_{2}$ :

$$
z_{2}^{\left(\mu_{2}^{1}\right)}(t)+a_{21} z_{2}^{\left(\mu_{2}^{1}-1\right)}(t)+\cdots+a_{2 \mu_{2}^{1}-1} z_{2}^{(1)}(t)+a_{2 \mu_{2}^{1}} z_{2}(t)=r_{2}(t)
$$

Furthermore, from equations of (2.4.40), the control law (2.4.49) can be realized using the state feedback structure:

$$
\begin{aligned}
\tau_{2}^{1} u(t)= & -D_{\mu_{2}^{1}} x(t)-a_{21} D_{\mu_{2}^{1}-1} x(t)-\cdots \\
& -a_{2 \mu_{2}^{1}-1} D_{1} x(t)-a_{2 \mu_{2}^{1}} D_{0} x(t)+r_{2}(t) .
\end{aligned}
$$

From (2.4.46) and (2.4.51), we obtain

$$
K_{p} u(t)=K_{0} x(t)+r(t)
$$

where $r(t)=\left[r_{1}(t), r_{2}(t)\right]^{T}, K_{p}=\left[\begin{array}{c}\tau_{1} \\ \tau_{2}^{1}\end{array}\right]$, and

$$
K_{0}=\left[\begin{array}{c}
-c_{1} A^{\mu_{1}}-a_{11} c_{1} A^{\mu_{1}-1}-\cdots-a_{1 \mu_{1}-1} c_{1} A-a_{1 \mu_{1}} c_{1} \\
-D_{\mu_{2}^{1}}-a_{21} D_{\mu_{2}^{1}-1}-\cdots-a_{2 \mu_{2}^{1}-1} D_{1}-a_{2 \mu_{2}^{1}} D_{0}
\end{array}\right]
$$

Since $K_{p}$ is nonsingular, we have the state feedback control law as

$$
u(t)=K_{p}^{-1} K_{0} x(t)+K_{p}^{-1} r(t)=K_{1}^{* T} x(t)+K_{2}^{*} r(t)
$$

where $K_{1}^{* T}=K_{p}^{-1} K_{0}$ and $K_{2}^{*}=K_{p}^{-1}$.

Applying the control law (2.4.54), the closed-loop system is decoupled into two subsystems given by (2.4.45) and (2.4.50), which can be expressed as

$$
\begin{aligned}
d_{1}(s)\left[y_{1}\right](t) & =r_{1}(t), \\
\bar{d}_{22}(s)\left[z_{2}\right](t) & =r_{2}(t) .
\end{aligned}
$$


Substituting (2.4.32) in (2.4.56), we have

$$
\xi_{m}(s)[y](t)=r(t)
$$

where $y(t)=\left[y_{1}(t), y_{2}(t)\right]^{T}$, and the modified left interactor matrix $\xi_{m}(s)$ is

$$
\xi_{m}(s)=\left[\begin{array}{cc}
d_{1}(s) & 0 \\
h_{21}^{m}(s) & d_{2}(s)
\end{array}\right]
$$

with $d_{2}(s)=\bar{d}_{21}(s) \bar{d}_{22}(s)$ and $h_{21}^{m}(s)=-\alpha_{21}^{1} \bar{h}_{2}(s) \bar{d}_{22}(s)$. Hence, the closed-loop system becomes

$$
y(t)=W_{m}(s)[r](t)
$$

where

$$
W_{m}(s)=\xi_{m}^{-1}(s)=\left[\begin{array}{cc}
\frac{1}{d_{1}(s)} & 0 \\
\frac{h_{21}^{m}(s)}{d_{1}(s) d_{2}(s)} & \frac{1}{d_{2}(s)}
\end{array}\right]
$$

which is stable as $d_{1}(s)$ and $d_{2}(s)$ are chosen to be stable. Note that this design procedure also defines a suitable modified left interactor matrix $\xi_{m}(s)$ from the knowledge of $\alpha_{21}, \mu_{1}, \mu_{2}$ and $\mu_{2}^{1}$ of the interactor matrix $\xi(s)$.

Remark 2.4.1. It is important to note that for internal stability of the closed-loop system, that is, all eigenvalues of $A+B K_{1}^{* T}$ are stable, we need the assumption that $(A, B, C)$ is stabilizable and detectable, in addition to the assumption that all zeros of the system transfer matrix $G(s)=C(s I-A)^{-1} B$ are stable (in the open left-half complex plane) (in other words, two assumptions together, all system zeros should be stable). Such conditions are for stable pole-zero cancelations in both the open-loop system $(A, B, C)$ and the closed-loop system transfer matrix $W_{m}(s)$.

\subsubsection{Output Feedback Control Design}

For the MIMO linear system (2.4.1), we employ an output feedback controller

$$
u(t)=\Theta_{1}^{* T} \frac{A(s)}{\Lambda(s)}[u](t)+\Theta_{2}^{* T} \frac{A(s)}{\Lambda(s)}[y](t)+\Theta_{20}^{*} y(t)+\Theta_{3}^{*} r(t)
$$


where $\Lambda(s)$ is a monic stable polynomial of degree $\nu-1$ for the observability index $\nu$ of the open-loop system, $A(s)=\left[I_{M}, s I_{M}, \ldots, s^{\nu-2} I_{M}\right]^{T}$, and $\Theta_{1}^{*} \in R^{M(\nu-1) \times M}$, $\Theta_{2}^{*} \in R^{M(\nu-1) \times M}, \Theta_{20}^{*} \in R^{M \times M}$ and $\Theta_{3}^{*} \in R^{M \times M}$ are some parameter matrices. It has been shown in [84] that there exist $\Theta_{1}^{*}, \Theta_{2}^{*}, \Theta_{20}^{*}$, and $\Theta_{3}^{*}$ satisfying the following plant-model matching condition:

$$
I_{M}-\Theta_{1}^{* T} F(s)-\left(\Theta_{2}^{* T} F(s)+\Theta_{20}^{*}\right) G(s)=\Theta_{3}^{*} W_{m}^{-1}(s) G(s), \Theta_{3}^{*}=K_{p}^{-1}, W_{m}=\xi_{m}^{-1}(s),
$$

with $F(s)=\frac{A(s)}{\Lambda(s)}$. Then, operating both sides of (2.4.62) on $u(t)$, from the system model $y(t)=G(s)[u](t)$, and the matching condition (2.4.62), we have

$$
y(t)=W_{m}(s)[r](t)=\xi_{m}^{-1}(s)[r](t)
$$

That is the output feedback controller (2.4.61) can achieve plant-model matching. 


\section{Chapter 3}

\section{Multivariable State Feedback Output Tracking MRAC}

This chapter presents a novel multivariable model reference adaptive control (MRAC) design using state feedback for achieving output tracking for uncertain multi-input and multi-output (MIMO) systems. Several key technical issues of the multivariable MRAC are addressed:

- analysis of restrictiveness of plant-model matching conditions;

- design of stable adaptive laws;

- closed-loop system stability and output tracking analysis; and

- design verification using the high-fidelity aircraft model-GTM.

State feedback control design is widely used for applications with measurable state signals, such as within aircraft flight control systems, due to its simple controller structure. For the state feedback state tracking MRAC design, the crucial plant-model matching condition is restrictive with respect to system uncertainties. In this chapter, for the state feedback output tracking MRAC design, a less restrictive matching condition, which can be satisfied under system parametric and structural uncertainties, will 
be derived. With such a less restrictive plant-model matching condition, a parameterized error model, based on a high frequency gain matrix decomposition that reduces a priori system knowledge, will be developed for design of stable adaptive laws. The state feedback controller updated by the adaptive laws can ensure closed-loop system stability and asymptotic output tracking. This multivariable MRAC scheme with the less restrictive matching condition provides a foundation for development of the adaptive fault-tolerant control.

\subsection{Problem Statement}

Consider the MIMO linear time-invariant (LTI) system described by

$$
\dot{x}(t)=A x(t)+B u(t), y(t)=C x(t)
$$

where the system matrices $A \in R^{n \times n}, B \in R^{n \times M}$ and $C \in R^{M \times n}$ have parameter uncertainties, and $x(t) \in R^{n}, u(t) \in R^{M}$ and $y(t) \in R^{M}$ are the state, input and output vector signals. The control objective is to design a state feedback control law $u(t)$ to make all signals in the closed-loop system bounded and the output signal $y(t)$ asymptotically track a given reference signal $y_{m}(t)$ generated from a reference system

$$
y_{m}(t)=W_{m}(s)[r](t), W_{m}(s)=\xi_{m}^{-1}(s)
$$

where $r(t) \in R^{M}$ is a bounded reference input signal, and $\xi_{m}(s)$ defined in Lemma 2.3.1 is the modified left interactor matrix of the system transfer matrix $G(s)=$ $C(s I-A)^{-1} B$, which has a stable inverse, i.e., $W_{m}(s)$ is stable.

\subsubsection{Motivation}

The motivation of studying the multivariable MRAC with state feedback for output tracking come from the fact that, for many important applications, there are some 
shortcomings in the two existing types of multivariable MRAC schemes, namely, state feedback state tracking and output feedback output tracking designs.

State feedback for state tracking. For state feedback state tracking design, the adaptive control law is given as

$$
u(t)=K_{1}^{T}(t) x(t)+K_{2}(t) r(t)
$$

where $K_{1}(t) \in R^{n \times M}$ and $K_{2}(t) \in R^{M \times M}$ are the adaptively updated estimates of nominal parameters $K_{1}^{*}$ and $K_{2}^{*}$. Applying the adaptive control law (3.1.3) to the system (3.1.1), the state signal $x(t)$ can asymptotically track a reference state signal $x_{m}(t)$ generated from

$$
\dot{x}_{m}(t)=A_{m} x_{m}(t)+B_{m} r(t),
$$

where $A_{m} \in R^{n \times n}$ is stable. For such an adaptive control design, the nominal parameters $K_{1}^{*}$ and $K_{2}^{*}$ must be existed to satisfy the matching condition:

$$
A+B K_{1}^{* T}=A_{m}, \quad B K_{2}^{*}=B_{m} .
$$

However, when the system matrices $A$ and $B$ have uncertainties, i.e. entries of $A$ and $B$ are unknown, the nominal parameters $K_{1}^{*}$ and $K_{2}^{*}$ are hard to be solved from the matching condition (3.1.5) with the chosen reference system $\left(A_{m}, B_{m}\right)$. That is the matching condition (3.1.5) is restrictive for the system with parameter uncertainties. Then, we use an aircraft flight control example to show the restrictiveness of the matching condition (3.1.5).

Example 3.1.1. Consider a linearized lateral-directional dynamic model of a large transport airplane [85] described by

$$
\dot{x}=A x+B u, x=\left[v_{b}, p_{b}, r_{b}, \phi, \psi\right]^{T}, u=\left[d_{a}, d_{r}\right]^{T} .
$$


The system matrices $A$ and $B$ have uncertainties due to variations of the operating conditions, where the structures are given as

$$
A=\left[\begin{array}{rrrrr}
a_{11} & a_{12} & a_{13} & a_{14} & 0 \\
a_{21} & a_{22} & a_{23} & a_{24} & 0 \\
a_{31} & a_{32} & a_{33} & a_{34} & 0 \\
0 & 1 & 0 & 0 & 0 \\
0 & 0 & 1 & 0 & 0
\end{array}\right], \quad B=\left[\begin{array}{rr}
b_{11} & b_{12} \\
b_{21} & b_{22} \\
b_{31} & b_{32} \\
0 & 0 \\
0 & 0
\end{array}\right]
$$

with $a_{i j}, i=1,2,3, j=1,2,3,4$, and $b_{i j}, j=1,2,3, j=1,2$, being the unknown parameters. Denote $k_{1 i j}^{*}, i=1,2, \ldots, 5, j=1,2$, as the $(i, j)$ component of $K_{1}^{*}$ and $k_{2 i j}^{*}, i=1,2, j=1,2$, as the $(i, j)$ component of $K_{2}^{*}$. It follows that

$$
A+B K_{1}^{* T}=\left[\begin{array}{rrrrr}
a_{c 11} & a_{c 12} & a_{c 13} & a_{c 14} & a_{c 15} \\
a_{c 21} & a_{c 22} & a_{c 23} & a_{c 24} & a_{c 25} \\
a_{c 31} & a_{c 32} & a_{c 33} & a_{c 34} & a_{c 35} \\
0 & 1 & 0 & 0 & 0 \\
0 & 0 & 1 & 0 & 0
\end{array}\right]
$$

where $a_{c i j}=a_{i j}+b_{i 1} k_{1 j 1}^{*}+b_{i 2} k_{1 j 2}^{*}, i=1,2,3, j=1,2,3,4, a_{c i 5}=b_{i 1} k_{151}^{*}+b_{i 2} k_{152}^{*}$, and

$$
B K_{2}^{*}=\left[\begin{array}{rr}
b_{11} k_{211}^{*}+b_{12} k_{221}^{*} & b_{11} k_{212}^{*}+b_{12} k_{222}^{*} \\
b_{21} k_{211}^{*}+b_{22} k_{221}^{*} & b_{21} k_{212}^{*}+b_{22} k_{222}^{*} \\
b_{31} k_{211}^{*}+b_{32} k_{221}^{*} & b_{31} k_{212}^{*}+b_{32} k_{222}^{*} \\
0 & 0 \\
0 & 0
\end{array}\right] .
$$

In view of (3.1.8) and (3.1.9), with the given matrices $A_{m}$ and $B_{m}$, there may not exist the solutions of $K_{1}^{*}$ and $K_{2}^{*}$ for the matching condition (3.1.5).

When $A$ and $B$ are known, we may use LQR or pole placement designs to derive the controller parameters $K_{1}^{*}$ and $K_{2}^{*}$. Meanwhile, the reference system matrices $A_{m}$ and $B_{m}$ can be obtained. However, when $A$ and $B$ have parameter uncertainties, such as (3.1.7), it is hard to apply LQR or pole placement designs to obtain $A_{m}$ and $B_{m}$, and $K_{1}^{*}$ and $K_{2}^{*}$ may not exist for some chosen $A_{m}$ and $B_{m}$ as shown in Example 3.1.1. Thus, the matching condition (3.1.5) is restrictive, which requires exact knowledge of $A$ and $B$ to obtain $K_{1}^{*}$ and $K_{2}^{*}$. Hence, in the presence of uncertainties of $A$ and $B$, 
the unsolvable (restrictive) matching condition (3.1.5) makes it difficult to design an adaptive state feedback for state tracking control law (3.1.3). On the contrary, the matching condition of the state feedback for output tracking (to be developed) is less restrictive and needs less system parameter information.

Output feedback for output tracking. If the control objective is to make the output signal $y(t)$ of the system (3.1.1) track the reference signal $y_{m}(t)$ generated from (3.1.2), we can apply the output feedback multivariable MRAC scheme to avoid the restrictive matching condition. It is well-known that such an output feedback controller is given as

$$
u(t)=\Theta_{1}^{T}(t) \omega_{1}(t)+\Theta_{2}^{T}(t) \omega_{2}(t)+\Theta_{20}(t) y(t)+\Theta_{3}(t) r(t)
$$

where $\omega_{1}(t)=\frac{A_{0}(s)}{\Lambda(s)}[u](t)$ and $\omega_{2}(t)=\frac{A_{0}(s)}{\Lambda(s)}[y](t)$ for a monic and stable polynomial $\Lambda(s)$ of degree $\nu-1$ and $A_{0}(s)=\left[I_{M}, s I_{M}, \ldots, s^{\nu-2} I_{M}\right]^{T}$ with $\nu$ being the observability index of the system $G(s)=C(s I-A)^{-1} B$. The adaptively updated parameters $\Theta_{1}(t)$, $\Theta_{2}(t), \Theta_{20}(t)$ and $\Theta_{3}(t)$ are the estimates of the nominal parameters $\Theta_{1}^{*}, \Theta_{2}^{*}, \Theta_{20}^{*}$ and $\Theta_{3}^{*}$, which satisfy the matching equation (2.4.62). However, such an output feedback controller with filter $\frac{A_{0}(s)}{\Lambda(s)}$ is more complex than the state feedback controller structure (3.1.3). A comparison of complexity of output feedback for output tracking and state feedback for output tracking will be presented in Section 3.3.1.

In many applications, the system state variables are available and the control objective is to achieve output tracking (e.g., aircraft flight control). For such applications, an effective and simple controller structure is desirable. It is the goal of this chapter to develop a new multivariable MRAC with state feedback for output tracking, which can avoid both the strict matching conditions and the complicated output feedback controller structure. 


\subsubsection{Design Assumptions}

To proceed the design, we make the following assumptions about the system:

(A3.1) all zeros of $G(s)$ have negative real parts;

(A3.2) $G(s)$ has full rank and its modified left interactor matrix $\xi_{m}(s)$ is known;

(A3.3) all leading principal minors $\Delta_{i}, i=1,2, \ldots, M$, of the high frequency gain matrix $K_{p}$ are nonzero and their signs are known; and

(A3.4) $(A, B)$ is controllable and $(A, C)$ is observable.

Assumptions (A3.1) and (A3.4) are needed for stable plant-model matching, assumption (A3.2) is used to obtain the reference model (3.1.2), and assumption (A3.3) is used for designing adaptive laws.

Remark 3.1.1. Although the parameters in $A, B$, and $C$ have uncertainties, the interactor matrix $\xi_{m}(s)$ and signs of leading principal minors of $K_{p}$ may be obtained from the structure knowledge or physical meanings of matrices $A, B$, and $C$. Next, we will use a generic aircraft model to show that $\xi_{m}(s)$ and signs are accessible for the aircraft flight system in the presence of parametric uncertainties. Hence, the design assumptions are not restrictive for the aircraft flight control system.

\subsection{Controller Structure}

We employ a state feedback controller:

$$
u(t)=K_{1}^{T}(t) x(t)+K_{2}(t) r(t)
$$

where $K_{1}$ and $K_{2}$ are estimates of the unknown constant matrices $K_{1}^{*}$ and $K_{2}^{*}$, which satisfy the following plant-model matching condition (2.4.3):

$$
C\left(s I-A-B K_{1}^{* T}\right)^{-1} B K_{2}^{*}=W_{m}(s), W_{m}(s)=\xi_{m}^{-1}(s) .
$$


The existence of $K_{1}^{*}$ and $K_{2}^{*}$ has been shown in Section 2.4.1. This result is also stated in [59] in terms of $H(s)=\xi_{m}^{-1}(s)=W_{m}(s)$, the Hermite form of $G(s)$. In particular, when the interactor matrix $\xi_{m}(s)$ of $G(s)$ is in a diagonal form, the matching condition can be treated as a solution to a decoupling problem for multivariable systems, which is stated and solved in [22]. When the interactor matrix $\xi_{m}(s)$ is in a lower triangular form, [62] gives a method to solve $K_{1}^{*}$ and $K_{2}^{*}$ for the matching condition (3.2.2).

\subsubsection{Robustness to Parameter Uncertainties}

In each matching condition (3.1.5) and (3.2.2), there are three components: the system $(A, B, C)$, the reference system $\left(A_{m}, B_{m}\right)$ or $\xi_{m}^{-1}(s)$, and $\left(K_{1}^{*}, K_{2}^{*}\right)$. A key point of comparing these two matching conditions is to see whether the reference systems $\left(A_{m}, B_{m}\right)$ and $\xi_{m}^{-1}(s)$ can be obtained under system parameter uncertainty condition to ensure the existence of $K_{1}^{*}$ and $K_{2}^{*}$.

When the parameters in $A$ and $B$ have uncertainties, it is hard to obtain $\left(A_{m}, B_{m}\right)$ using LQR or pole-placement design, and $K_{1}^{*}$ and $K_{2}^{*}$ may not exist for some chosen $\left(A_{m}, B_{m}\right)$, since the matching condition (3.1.5) requires strict matrix matching condition, as explained in Section 3.1.1. On the contrary, we will show that, when the parameters in $A, B$, and $C$ have uncertainties, under some structural matrix non-singularity conditions, the reference system $\xi_{m}^{-1}(s)$ can be obtained, that is the matching condition (3.2.2) can be satisfied when $(A, B, C)$ has uncertainties.

To proceed for the system transfer matrix $G(s)=C(s I-A)^{-1} B$, denoting $C_{i}, i=$ $1,2, \ldots, M$, as the $i$ th row of $C$, the $i$ th row of $G(s)$ is given as

$$
G_{i}(s)=\frac{1}{\beta(s)}\left(E_{i(n-1)} s^{n-1}+\cdots+E_{i 1} s+E_{i 0}\right),
$$

where $\beta(s)=\operatorname{det}(s I-A)=s^{n}+\beta_{n-1} s^{n-1}+\cdots+\beta_{1} s+\beta_{0}, E_{i(n-1)}=C_{i} B, E_{i(n-2)}=$ $C_{i} A B+\beta_{n-1} C_{i} B, \ldots, E_{i 0}=C_{i} A^{n-1} B+\cdots+\beta_{2} C_{i} A B+\beta_{1} C_{i} B$. We define a set of indices 
$\left\{\rho_{1}, \rho_{2}, \ldots, \rho_{M}\right\}$, such that $C_{i} A^{\rho_{i}-1} B \neq 0$ and $C_{i} A^{k} B=0, k=0,1, \ldots, \rho_{i}-2$.

Proposition 3.2.1. If the matrix

$$
K_{p d}=\left[\begin{array}{c}
C_{1} A^{\rho_{1}-1} B \\
C_{2} A^{\rho_{2}-1} B \\
\vdots \\
C_{M} A^{\rho_{M}-1} B
\end{array}\right]
$$

has full rank, then the diagonal matrix

$$
\xi_{m}(s)=\operatorname{diag}\left\{d_{1}(s), d_{2}(s), \ldots, d_{M}(s)\right\}
$$

where $d_{i}(s), i=1,2, \ldots, M$, is a monic stable polynomial of degree $\rho_{i}$, is the left modified interactor matrix, and the high frequency gain matrix is $K_{p}=K_{p d}$.

Proof: From (3.2.3) and the definition of index $\rho_{i}$, it can be easily shown that $\lim _{s \rightarrow \infty} \xi_{m}(s) G(s)=K_{p d}$. Since $K_{p d}$ has full rank, from the definition in Lemma 2.3.1, $\xi_{m}(s)$ is the interactor matrix, and $K_{p d}$ is the high frequency gain matrix. $\nabla$ Under the uncertainties of $A, B$, and $C$, if the matrix $K_{p d}$ defined in (3.2.4) is nonsingular, the diagonal $\xi_{m}(s)(3.2 .5)$ is the system interactor matrix and the reference system $W_{m}(s)=\xi_{m}^{-1}(s)$ is available for solving the matching condition (3.2.2). Therefore, such a matrix non-singularity requirement is much less restrictive than the strict matching condition required in the state matching equation (3.1.5).

Proposition 3.2.2. If the matrix $K_{p d}$ defined in (3.2.4) is singular, then the interactor matrix $\xi_{m}(s)$ is of lower triangular form (2.3.1) [89].

Reference [89] gives a constructive procedure to build such a lower triangular interactor matrix. To obtain the $\xi_{m}(s)$, we may need more prior structural knowledge, but the conditions used in [89] may be still less constrained than knowing exact $A$ and $B$ for strict matrix matching condition (3.1.5). 
Robustness of interactor matrix. In real life applications, the structures of $A$, $B$, and $C$ may be known (some elements may be zeros), and the uncertain parameters may have bounds. Based on such structural knowledge of the system, we could obtain the interactor matrix $\xi_{m}(s)$, which may be of diagonal form if $K_{p d}$ has full rank or lower triangular form if $K_{p d}$ is singular, then the nonsingular high frequency gain matrix $K_{p}$ can also be obtained. When parameters change within the physical bounds, the probability of $K_{p}$ being singular is very small, since the parameters which makes the determinant of $K_{p}$ be zero lies in a low-dimensional manifold. Hence, the chosen $\xi_{m}(s)$ is still the system interactor matrix.

Next, we will use an aircraft model with parameter uncertainties to show that the interactor matrix $\xi_{m}(s)$ can be obtained and the high frequency gain matrix $K_{p}$ remains nonsingular with the chosen $\xi_{m}(s)$.

\subsubsection{An Aircraft Example}

Recall that the linear aircraft lateral-directional dynamic model is

$$
\dot{x}(t)=A x(t)+B u(t), \quad y(t)=C x(t),
$$

where the generic structures of $A$ and $B$ are given in (3.1.7). Here, we choose the roll angle $\phi$ and the yaw angle $\psi$ as the outputs, so the $C$ matrix is

$$
C=\left[\begin{array}{lllll}
0 & 0 & 0 & 1 & 0 \\
0 & 0 & 0 & 0 & 1
\end{array}\right]
$$

From (3.2.3), we have the transfer matrix is $G(s)=\left[G_{1}(s), G_{2}(s)\right]^{T}$, with

$$
\begin{aligned}
& G_{1}(s)=\frac{1}{\beta(s)}\left(E_{13} s^{3}+\cdots+E_{11} s+E_{10}\right), \\
& G_{2}(s)=\frac{1}{\beta(s)}\left(E_{23} s^{3}+\cdots+E_{21} s+E_{20}\right),
\end{aligned}
$$

where $E_{13}=C_{1} A B=\left[b_{21}+\tan \theta_{0} b_{31}, b_{22}+\tan \theta_{0} b_{32}\right] \neq 0$ and $E_{23}=C_{2} A B=$ $\left[\frac{1}{\cos \theta_{0}} b_{31}, \frac{1}{\cos \theta_{0}} b_{32}\right] \neq 0$, since $b_{21}, b_{22}, b_{31}$, and $b_{32}$ are control gains from the corre- 
sponding control surfaces to the state accelerations and the signs are known: $b_{21}<0$, $b_{22}>0, b_{31}<0$, and $b_{32}<0$. So the determinant of

$$
K_{p d}=\left[\begin{array}{c}
C_{1} A B \\
C_{2} A B
\end{array}\right]=\left[\begin{array}{cc}
b_{21}+\tan \theta_{0} b_{31} & b_{22}+\tan \theta_{0} b_{32} \\
\frac{1}{\cos \theta_{0}} b_{31} & \frac{1}{\cos \theta_{0}} b_{32}
\end{array}\right]
$$

is given as $\operatorname{det}\left(K_{p d}\right)=\frac{1}{\cos \theta_{0}}\left(b_{32} b_{21}-b_{31} b_{22}\right) \neq 0$. From Proposition 3.2.1, the interactor matrix for the system (3.2.6) can be chosen as $\xi_{m}(s)=\operatorname{diag}\left\{(s+1)^{2},(s+1)^{2}\right\}$ and the high frequency gain matrix $K_{p}=K_{p d}$. Thus, for the generic lateral-directional aircraft model (3.2.6) with parameters changing within the physical range, the reference transfer matrix can be chosen as $W_{m}(s)=\xi_{m}^{-1}(s)$, and the matching condition (3.2.2) can be satisfied. On the other hand, the matching condition (3.1.5) cannot be satisfied for the generic lateral-directional aircraft model (3.2.6) as in Section 3.1.1.

\subsection{Parameter Adaptation Scheme}

In this section, we present the design and analysis of an adaptive scheme for the control law $u(t)$ in (3.2.1). Substituting the control law (3.2.1) in (3.1.1), we have

$$
\begin{aligned}
& \dot{x}(t)=\left(A+B K_{1}^{* T}\right) x(t)+B K_{2}^{*} r(t)+B\left(\left(K_{1}^{T}(t)-K_{1}^{* T}\right) x(t)+\left(K_{2}(t)-K_{2}^{*}\right) r(t)\right), \\
& y(t)=C x(t) .
\end{aligned}
$$

In view of the reference model (3.1.2), matching condition (3.2.2) and (3.3.1), the output tracking error $e(t)=y(t)-y_{m}(t)$ is

$$
e(t)=W_{m}(s) K_{p}\left[\widetilde{\Theta}^{T} \omega\right](t)+C e^{\left(A+B K_{1}^{* T}\right) t} x(0),
$$

where $C e^{\left(A+B K_{1}^{* T}\right) t} x(0)$ converges to zero exponentially due to the stability of $A+$ $B K_{1}^{* T}, \widetilde{\Theta}(t)=\Theta(t)-\Theta^{*}, \Theta(t)=\left[K_{1}^{T}(t), K_{2}(t)\right]^{T}, \Theta^{*}=\left[K_{1}^{* T}, K_{2}^{*}\right]^{T}, \omega(t)=\left[x^{T}, r^{T}\right]^{T}$. If the control law (3.2.1) is implemented with $K_{1}(t)=K_{1}^{*}$ and $K_{2}(t)=K_{2}^{*}$, we would have $\lim _{t \rightarrow \infty}\left(y(t)-y_{m}(t)\right)=0$. However, $K_{1}^{*}$ and $K_{2}^{*}$ are unknown, we need to use the $K_{1}(t)$ and $K_{2}(t)$ updated from some adaptive laws to be developed next. 


\subsubsection{Design Based on the LDS Decomposition}

To design adaptive parameter update laws, it is crucial to develop an error model in terms of some related parameter errors and the tracking error $e(t)$.

Error model development. From the tracking error (3.3.2), ignoring the exponentially decaying term $C e^{\left(A+B K_{1}^{* T}\right) t} x(0)$, we obtain

$$
\xi_{m}(s)[e](t)=K_{p} \widetilde{\Theta}^{T}(t) \omega(t) .
$$

To deal with the uncertainty of $K_{p}$, we use its LDS decomposition defined in (2.3.5): $K_{p}=L_{s} D_{s} S$. Substituting (2.3.5) in (3.3.3), we obtain

$$
L_{s}^{-1} \xi_{m}(s)[e](t)=D_{s} S \widetilde{\Theta}^{T}(t) \omega(t)
$$

To parameterize the unknown matrix $L_{s}$, we introduce

$$
\Theta_{0}^{*}=L_{s}^{-1}-I=\left\{\theta_{i j}^{*}\right\}
$$

where $\theta_{i j}^{*}=0$ for $i=1,2, \ldots, M$ and $j \geq i$. We have

$$
\xi_{m}(s)[e](t)+\Theta_{0}^{*} \xi_{m}(s)[e](t)=D_{s} S \widetilde{\Theta}^{T}(t) \omega(t) .
$$

We introduce a filter $h(s)=1 / f_{h}(s)$, where $f_{h}(s)$ is a stable and monic polynomial whose degree equals the degree of $\xi_{m}(s)$. Operating (3.3.6) by $h(s) I_{M}$ leads to

$$
\bar{e}(t)+\left[0, \theta_{2}^{* T} \eta_{2}(t), \theta_{3}^{* T} \eta_{3}(t), \ldots, \theta_{M}^{* T} \eta_{M}(t)\right]^{T}=D_{s} S h(s)\left[\widetilde{\Theta}^{T} \omega\right](t),
$$

where

$$
\begin{aligned}
\bar{e}(t) & =\xi_{m}(s) h(s)[e](t)=\left[\bar{e}_{1}(t), \ldots, \bar{e}_{M}(t)\right]^{T}, \\
\eta_{i}(t) & =\left[\bar{e}_{1}(t), \ldots, \bar{e}_{i-1}(t)\right]^{T} \in R^{i-1}, i=2, \ldots, M, \\
\theta_{i}^{*} & =\left[\theta_{i 1}^{*}, \ldots, \theta_{i i-1}^{*}\right]^{T}, i=2, \ldots, M .
\end{aligned}
$$


Based on this parameterized error equation, we introduce the estimation error

$$
\epsilon(t)=\left[0, \theta_{2}^{T}(t) \eta_{2}(t), \theta_{3}^{T}(t) \eta_{3}(t), \ldots, \theta_{M}^{T}(t) \eta_{M}(t)\right]^{T}+\Psi(t) \xi(t)+\bar{e}(t)
$$

where $\Psi(t)$ is the estimate of $\Psi^{*}=D_{s} S$, and

$$
\xi(t)=\Theta^{T}(t) \zeta(t)-h(s)\left[\Theta^{T} \omega\right](t), \zeta(t)=h(s)[\omega](t) .
$$

From (3.3.7)-(3.3.12), we can derive the following equation:

$$
\epsilon(t)=\left[0, \tilde{\theta}_{2}^{T}(t) \eta_{2}(t), \tilde{\theta}_{3}^{T}(t) \eta_{3}(t), \ldots, \tilde{\theta}_{M}^{T}(t) \eta_{M}(t)\right]^{T}+D_{s} S \tilde{\Theta}(t)^{T} \zeta(t)+\tilde{\Psi}(t) \xi(t)
$$

where $\tilde{\theta}_{i}(t)=\theta_{i}(t)-\theta_{i}^{*}, i=2,3, \ldots, M$, and $\widetilde{\Psi}(t)=\Psi(t)-\Psi^{*}$.

Adaptive laws. With the estimation error (3.3.13), we choose the adaptive laws

$$
\begin{aligned}
\dot{\theta}_{i}(t) & =-\frac{\Gamma_{\theta i} \epsilon_{i}(t) \eta_{i}(t)}{m^{2}(t)}, i=2,3, \ldots, M, \\
\dot{\Theta}^{T}(t) & =-\frac{D_{s} \epsilon(t) \zeta^{T}(t)}{m^{2}(t)} \\
\dot{\Psi}(t) & =-\frac{\Gamma \epsilon(t) \xi^{T}(t)}{m^{2}(t)},
\end{aligned}
$$

where the signal $\epsilon(t)=\left[\epsilon_{1}(t), \epsilon_{2}(t), \ldots, \epsilon_{M}(t)\right]^{T}$ is computed from $(3.3 .11), \Gamma_{\theta i}=\Gamma_{\theta i}^{T}>$ $0, i=2,3, \ldots, M$, and $\Gamma=\Gamma^{T}>0$ are adaptation gain matrices, and

$$
m(t)=\left(1+\zeta^{T}(t) \zeta(t)+\xi^{T}(t) \xi(t)+\sum_{i=2}^{M} \eta_{i}^{T}(t) \eta_{i}(t)\right)^{1 / 2}
$$

Complexity analysis. First, we note that the state feedback controller structure (3.2.1) is less complex than the output feedback controller structure (3.1.10). Since a matrix filter $F(s)$ is used in the output feedback controller (3.1.10), it is necessary to use additional integrators. Another comparison is based on number of updated parameters and number of filtered signals.

Number of updated parameters. The number of parameters updated in the state feedback for output tracking adaptive laws (3.3.14)-(3.3.16) is $N_{s}=\frac{M^{2}-M}{2}+$ 
$(n+M) M+M^{2}$, and the number for the output feedback for output tracking adaptive laws is $N_{o}=\frac{M^{2}-M}{2}+(2 \nu+1) M^{2}$. From the observability index $\nu$ definition [84], we have $\nu M \geq n$. Then, we can derive that

$$
N_{o}-N_{s}=2 \nu M^{2}-n M-M^{2} \geq(n-M) M \geq 0 .
$$

That is, the state feedback for output tracking design has fewer parameters to be updated than the output feedback for output tracking design (note that $N_{o}=N_{s}$ only when $\nu=1$ and $n=M)$.

Number of filtered signals. Letting $n_{h}^{*}=\operatorname{deg}\left(f_{h}(s)\right)$, the number of integrators in construction of the filtered signals $\xi(t)$ and $\zeta(t)$ used in state feedback for output tracking is $n_{h}^{*}(2 M+n)$, and the number of integrators for $\bar{e}(t)$ is $n_{\bar{e}}^{*}$ that is related to the filter $\xi_{m}(s) h(s)$. So the total number of integrators for the state feedback output tracking is given by $N_{f s}=n_{h}^{*}(2 M+n)+n_{\bar{e}}^{*}$. Similarly, the number of integrators used in output feedback for output tracking is given by $N_{f o}=n_{h}^{*}(2 \nu M+M)+n_{\bar{e}}^{*}$. From $\nu M \geq n$, we have

$$
N_{f o}-N_{f s}=n_{h}^{*}(2 \nu M-n-M) \geq n_{h}^{*}(n-M) \geq 0 .
$$

That is, the state feedback for output tracking design has fewer integrators than the output feedback for output tracking design (as in the case of number of parameters to be updated, $N_{f o}=N_{f s}$ only when $\nu=1$ and $\left.n=M\right)$.

Based on the above analysis, we have the state feedback for output tracking scheme is simpler than the output feedback for output tracking scheme.

\subsubsection{Stability Analysis}

For the adaptive laws (3.3.14)-(3.3.16), we have following desired properties.

Lemma 3.3.1. The adaptive laws (3.3.14)-(3.3.16) ensure that 
(i) $\theta_{i}(t) \in L^{\infty}, i=2,3, \ldots, M, \Theta(t) \in L^{\infty}, \Psi(t) \in L^{\infty}$, and $\frac{\epsilon(t)}{m(t)} \in L^{2} \cap L^{\infty}$;

(ii) $\dot{\theta}_{i}(t) \in L^{2} \cap L^{\infty}, i=2,3, \ldots, M, \dot{\Theta}(t) \in L^{2} \cap L^{\infty}$, and $\dot{\Psi}(t) \in L^{2} \cap L^{\infty}$.

Proof: Consider the positive definite function

$$
V=\frac{1}{2}\left(\sum_{i=2}^{M} \tilde{\theta}_{i}^{T} \Gamma_{\theta i}^{-1} \tilde{\theta}_{i}+\operatorname{tr}\left[\widetilde{\Psi}^{T} \Gamma^{-1} \widetilde{\Psi}\right]+\operatorname{tr}\left[\widetilde{\Theta} S \widetilde{\Theta}^{T}\right]\right) .
$$

From (3.3.14)-(3.3.16), we derive the time-derivative of $V$

$$
\dot{V}=-\sum_{i=2}^{M} \frac{\tilde{\theta}_{i}^{T} \epsilon_{i}(t) \eta_{i}(t)}{m^{2}(t)}-\frac{\xi^{T}(t) \widetilde{\Psi}^{T} \epsilon(t)}{m^{2}(t)}-\frac{\zeta^{T}(t) \widetilde{\Theta} S D_{s} \epsilon(t)}{m^{2}(t)}=-\frac{\epsilon^{T}(t) \epsilon(t)}{m^{2}(t)} \leq 0 .
$$

From (3.3.20), we have $\theta_{i}(t) \in L^{\infty}, i=2,3, \ldots, M, \Theta(t) \in L^{\infty}, \Psi(t) \in L^{\infty}, \frac{\epsilon(t)}{m(t)} \in$ $L^{2} \cap L^{\infty}, \dot{\theta}_{i}(t) \in L^{2} \cap L^{\infty}, i=2,3, \ldots, M, \dot{\Theta}(t) \in L^{2} \cap L^{\infty}$, and $\dot{\Psi}(t) \in L^{2} \cap L^{\infty} . \quad \nabla$

Based on Lemma 3.3.1, we can prove the following system stability property.

Theorem 3.3.1. The multivariable MRAC scheme with the state feedback controller (3.2.1) updated by the adaptive laws (3.3.14)-(3.3.16), when applied to (3.1.1), guarantees the closed-loop signal boundedness and asymptotic output tracking: $\lim _{t \rightarrow \infty}(y(t)-$ $\left.y_{m}(t)\right)=0$, for any initial conditions.

The proof of Theorem 3.3.1 can be carried out by using a similar way as described in [46] and [84] for multivariable MRAC using output feedback (see (3.1.10)). A key step of such an analysis procedure is to express a filtered version of the plant output $y(t)$ in a feedback framework which has a small gain due to the $L^{2}$ properties of $\dot{\Theta}(t)$, $\dot{\theta}_{i}(t)$ and $\frac{\epsilon(t)}{m(t)}$. Since the state feedback control signal $u(t)$ depends on the state $x(t)$, we need to express it in terms of the output $y(t)$ (and the input $u(t)$ itself through a dynamic block). This can be done using a state observer representation $\dot{x}=A x+B u$, $y=C x: \dot{x}=(A-L C) x+B u+L y$ for a gain matrix $L \in R^{n \times M}$ such that $A-L C$ is stable (which is possible because $(A, C)$ is observable). Then, the analysis procedure in [84] can be used to conclude the closed-loop signal boundedness and asymptotic 
output tracking: $\lim _{t \rightarrow \infty}\left(y(t)-y_{m}(t)\right)=0$ for the state feedback case. The detailed proof has been shown for the discrete-time adaptive control design in Chapter 7.

\subsection{Control Issues of Nonlinear Systems}

The commonly used method of control design for nonlinear systems (such as aircraft flight control systems) is based on linearization of the nonlinear systems. In this section, we will present the procedure of applying the state feedback for output tracking MRAC design to the nonlinear systems.

\subsubsection{Control Design}

The nonlinear system can be denoted as

$$
\dot{x}(t)=f_{n}(x(t), u(t)), \quad y(t)=h(x(t)),
$$

where $x \in R^{n}, u \in R^{M}$ and $y \in R^{M}$ are the state, input and output signals.

Linearization. Applying the Taylor series expansion at an equilibrium point $\left(x_{0}, u_{0}\right)\left(f_{n}\left(x_{0}, u_{0}\right)=0\right)$, we have the nonlinear system as

$$
\begin{aligned}
\Delta \dot{x}(t) & =\dot{x}(t)=A \Delta x(t)+B \Delta u(t)+R_{n}(\Delta x, \Delta u), \\
\Delta y(t) & =C \Delta x(t)+R_{h}(\Delta x),
\end{aligned}
$$

where $\Delta x(t)=x(t)-x_{0}, \Delta u(t)=u(t)-u_{0}, \Delta y(t)=y(t)-h\left(x_{0}\right), R_{n}(\Delta x, \Delta y)$ and $R_{h}(\Delta x)$ are the higher-order terms, and

$$
A=\left.\frac{\partial f_{n}}{\partial x}\right|_{\left(x_{0}, u_{0}\right)}, B=\left.\frac{\partial f_{n}}{\partial u}\right|_{\left(x_{0}, u_{0}\right)}, C=\left.\frac{\partial h}{\partial x}\right|_{x_{0}} .
$$

In a small neighborhood of $\left(x_{0}, u_{0}\right)$, we approximate the nonlinear system as

$$
\Delta \dot{x}(t)=A \Delta x(t)+B \Delta u(t), \quad \Delta y(t)=C \Delta x(t) .
$$




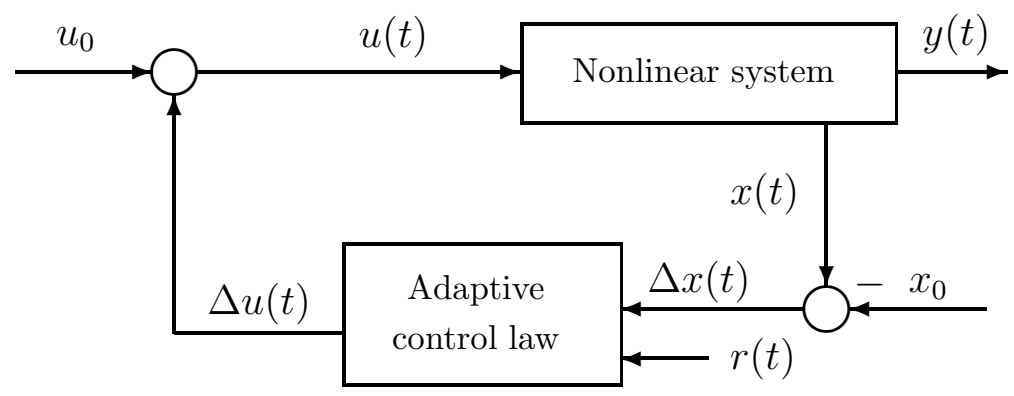

Figure 3.1: Closed-loop system with nonlinear system.

Design objective. The output perturbation $\Delta y(t)$ should track a given reference signal $\Delta y_{m}(t)$

$$
\Delta y_{m}(t)=W_{m}(s)[r](t), W_{m}(s)=\xi_{m}^{-1}(s),
$$

where $r(t)$ is a bounded reference input signal, and $\xi_{m}(s)$ is a modified left interactor matrix of the transfer matrix $G(s)=C(s I-A)^{-1} B$.

Linear control law design. Based on the linearized system (3.4.4), we generate the MRAC signal $\Delta u(t)$ as

$$
\Delta u(t)=K_{1}^{T}(t) \Delta x(t)+K_{2}(t) r(t)
$$

where $K_{1}(t)$ and $K_{2}(t)$ are updated from (3.3.14)-(3.3.16).

Control law for the nonlinear system. We add the equilibrium signal $u_{0}$ with $\Delta u(t)$ to obtain the controller signal $u(t)=\Delta u(t)+u_{0}$ and apply it to the nonlinear system (3.4.1). Figure 3.1 shows the closed-loop system.

Compensation of approximation errors. The developed adaptive control design is based on the linearized system without the approximation errors $R_{n}$ and $R_{h}$. When applied to the nonlinear system, the approximation errors could be compensated when they are small and approximate output tracking could be achieved in an average sense. However, the exact asymptotic output tracking might not take place, even if the perturbations are small, since the perturbations could lead to some resid- 
ual errors, or even parameter drift or instability. To avoid parameter drift, standard robust modifications of the adaptive laws can be used.

For a comparison study, we will also apply a fixed controller, which is the nominal controller $\Delta u(t)=K_{1}^{* T} \Delta x(t)+K_{2}^{*} \Delta r(t)$, to the nonlinear system. The simulation result will show that, for a same reference input $r(t)$, the adaptive controller can compensate the approximation errors to achieve approximate output tracking, while the fixed controller cannot compensate the errors. Thus, we may conclude that operation range of the linearization-based adaptive controller is larger than the fixed controller.

\subsubsection{System Infinite Zero Structure}

Consider a control-affine nonlinear system

$$
\dot{x}(t)=f(x(t))+\sum_{i=1}^{M} g_{i}(x(t)) u_{i}(t), y(t)=h(x(t)),
$$

where $h(x(t))=\left[h_{1}(x(t)), h_{2}(x(t)), \ldots, h_{M}(x(t))\right]^{T}$. Assume that the system (3.4.7) has a relative degree $\left\{\rho_{1}, \rho_{2}, \ldots, \rho_{M}\right\}$ at a neighborhood of $x_{0}$, such that (i) $L_{g_{j}} L_{f}^{k} h_{i}(x)=$ $0, \forall 1 \leq j \leq M, k<\rho_{i}-1,1 \leq i \leq M$, and (ii) the matrix

$$
\alpha(x)=\left[\begin{array}{ccc}
L_{g_{1}} L_{f}^{\rho_{1}-1} h_{1}(x) & \cdots & L_{g_{M}} L_{f}^{\rho_{1}-1} h_{1}(x) \\
L_{g_{1}} L_{f}^{\rho_{2}-1} h_{2}(x) & \cdots & L_{g_{M}} L_{f}^{\rho_{2}-1} h_{2}(x) \\
\vdots & \vdots & \vdots \\
L_{g_{1}} L_{f}^{\rho_{M}-1} h_{M}(x) & \cdots & L_{g_{M}} L_{f}^{\rho_{M}-1} h_{M}(x)
\end{array}\right]
$$

is nonsingular, for $\forall x$ in the neighborhood of $x_{0}$ [43]. Here, $L_{g_{j}} L_{f}^{k} h_{i}(x), i=1,2, \ldots, M, j=$ $1,2, \ldots, M, k=0,1, \ldots, \rho_{\imath}-1$, denotes $L_{g_{j}} L_{f}^{k} h_{i}(x)=\frac{\partial L_{f}^{k} h_{i}}{\partial x} g_{j}$, where $L_{f}^{k} h_{i}=L_{f}\left(L_{f}^{k-1} h_{i}\right)=$ $\frac{\partial L_{f}^{k-1} h}{\partial x} f, \ldots, L_{f}^{0} h_{i}=h_{i}$.

Linearization. We now linearize the system (3.4.7) at an equilibrium $\left(x_{0}, u_{0}\right)$, such that $f\left(x_{0}\right)=0$ and $u_{0}=\left[u_{1_{0}}, u_{2_{0}}, \ldots, u_{M_{0}}\right]^{T}=0$. From (3.4.4) and (3.4.3), we obtain the linearized system of (3.4.7) as

$$
\Delta \dot{x}(t)=A \Delta x(t)+B \Delta u(t), \quad \Delta y(t)=C \Delta x(t),
$$


where $\Delta x(t)=x(t)-x_{0}, \Delta u(t)=u(t), \Delta y(t)=y(t)-h\left(x_{0}\right)$,

$$
A=\left.\frac{\partial f}{\partial x}\right|_{x_{0}}, B=\left[g_{1}\left(x_{0}\right), \ldots, g_{M}\left(x_{0}\right)\right], C=\left[\left.\frac{\partial h_{1}}{\partial x}\right|_{x_{0}}, \ldots,\left.\frac{\partial h_{M}}{\partial x}\right|_{x_{0}}\right]^{T}
$$

Theorem 3.4.1. If the relative degree of nonlinear system (3.4.7) is $\left\{\rho_{1}, \ldots, \rho_{M}\right\}$, then the interactor matrix of the linearized system (3.4.9) is of the diagonal form: $\xi_{m}(s)=\operatorname{diag}\left\{d_{1}(s), d_{2}(s), \ldots, d_{M}(s)\right\}$, where $d_{i}(s), i=1,2, \ldots, M$, are monic stable polynomials of degrees $l_{i}=\rho_{i}$.

The above result is derived for the case when the nonlinear system (3.4.7) has a set of well-defined relative degrees $\rho_{i}, i=1,2, \ldots, M$, for $x$ in a neighborhood of $x_{0}$, which leads to a diagonal interactor matrix (or Hermite form) of the linearized system obtained at $x_{0}$. This implies that the interactor matrix of the linearized system obtained in a neighborhood of $x_{0}$ does not change. This is an important system invariance under linearization. This property is crucial for application of multivariable MRAC to nonlinear systems.

\subsection{Application to the GTM}

In this section, we apply the linearization-based multivariable MRAC design to the NASA generic transport model (GTM), to assess its effectiveness for the nonlinear aircraft system.

\subsubsection{The NASA Generic Transport Model (GTM)}

To test the MRAC scheme, we will use the nonlinear Simulink model of the GTM developed by NASA, which represents realistic flight scenarios, to perform simulation studies of our adaptive control scheme. The GTM is a nonlinear system denoted as

$$
\dot{x}_{g}(t)=f\left(x_{g}(t), u_{g}(t)\right), \quad y_{g}(t)=h\left(x_{g}(t)\right)
$$


where the output $y_{g}$ is a part of the state $x_{g}$, and the state and the input are

$$
x_{g}=\left[u_{b}, w_{b}, q_{b}, \theta, v_{b}, p_{b}, r_{b}, \phi, \psi\right]^{T}, u_{g}=\left[d_{e}, d_{a}, d_{r}, d_{t}\right]^{T}
$$

Under a wings-level flight condition, the aircraft can be decoupled into lateral-directional and longitudinal dynamics.

\subsubsection{Control of the GTM}

In this simulation study, we will apply the developed linearization-based design to the linearized and nonlinear GTMs. After linearizing the GTM model (3.5.1) at an equilibrium point (steady straight wings-level flight at 90 knots) $\left(x_{g 0}, u_{g 0}\right)$, the longitudinal and the lateral-directional dynamics are decoupled, and we only consider the linearized lateral-directional dynamics:

$$
\Delta \dot{x}(t)=A \Delta x(t)+B \Delta u(t), \quad \Delta y(t)=C \Delta x(t),
$$

where $\Delta x(t)=x(t)-x_{0}, \Delta u(t)=u(t)-u_{0}, \Delta y(t)=y(t)-C x_{0}, x(t)$ and $u(t)$ are

$$
x(t)=\left[v_{b}, p_{b}, r_{b}, \phi, \psi\right]^{T}, u(t)=\left[d_{a}, d_{r}\right]^{T},
$$

and we choose $\phi$ and $\psi$ as the output signals

$$
y(t)=[\phi, \psi]^{T}=C x(t)
$$

such that

$$
C=\left[\begin{array}{lllll}
0 & 0 & 0 & 1 & 0 \\
0 & 0 & 0 & 0 & 1
\end{array}\right]
$$

For the lateral-directional dynamics (3.5.2), the structures of $A$ and $B$ are

$$
A=\left[\begin{array}{rrrrr}
a_{11} & a_{12} & a_{13} & a_{14} & 0 \\
a_{21} & a_{22} & a_{23} & 0 & 0 \\
a_{31} & a_{32} & a_{33} & 0 & 0 \\
0 & 1 & \tan \theta_{0} & 0 & 0 \\
0 & 0 & \frac{1}{\cos \theta_{0}} & 0 & 0
\end{array}\right], \quad B=\left[\begin{array}{rr}
b_{11} & b_{12} \\
b_{21} & b_{22} \\
b_{31} & b_{32} \\
0 & 0 \\
0 & 0
\end{array}\right],
$$

where $\theta_{0}$ is the equilibrium point of the pitch angle. 
Remark 3.5.1. Since the linearized model of the nonlinear system (3.5.1) is obtained for a neighborhood of $\left(x_{g 0}, u_{g 0}\right)$, the lateral-directional dynamics of the GTM model can be treated as decoupled from the longitudinal dynamics around $\left(x_{g 0}, u_{g 0}\right)$. Then, the nonlinear lateral-directional dynamics equation may be expressed as

$$
\dot{x}=f_{l}(x)+\left[g_{l 1}(x), g_{l 2}(x)\right] u, \quad y=[\phi, \psi]^{T},
$$

around its equilibrium $\left(x_{0}, 0\right)$, whose linearized system is (3.5.2). Based on Theorem 3.3.1, for the lateral-directional dynamics, the system infinite zero structure is invariant. This implies that the linearization-based design is meaningful in terms of the design condition of the invariant infinite zero structure, when applied to the nonlinear GTM for its lateral-directional dynamics control.

Verification of design conditions. The transfer matrix of the system (3.5.2) is $G(s)=\left[G_{1}(s), G_{2}(s)\right]^{T}$, where $G_{1}(s)=\frac{1}{\beta(s)}\left(E_{13} s^{3}+\cdots+E_{11} s+E_{10}\right), G_{2}(s)=$ $\frac{1}{\beta(s)}\left(E_{23} s^{3}+\cdots+E_{21} s+E_{20}\right)$, with

$$
\begin{aligned}
& E_{13}=C_{1} A B=\left[b_{21}+\tan \theta_{0} b_{31}, b_{22}+\tan \theta_{0} b_{32}\right] \neq 0 \\
& E_{23}=C_{2} A B=\left[\frac{1}{\cos \theta_{0}} b_{31}, \frac{1}{\cos \theta_{0}} b_{32}\right] \neq 0
\end{aligned}
$$

Based on the values of $A$ and $B$ obtained from the GTM model, it can be verified that all zeros of $G(s)$ have negative real parts and $G(s)$ has full rank, and the matrix $\left[E_{13}^{T}, E_{23}^{T}\right]^{T}$ is nonsingular. From Proposition 3.2.1, we choose

$$
\xi_{m}(s)=\operatorname{diag}\left\{(s+1)^{2},(s+1)^{2}\right\}
$$

then the high frequency gain matrix is given as

$$
K_{p}=\left[\begin{array}{l}
C_{1} A B \\
C_{2} A B
\end{array}\right]=\left[\begin{array}{cc}
b_{21}+\tan \theta_{0} b_{31} & b_{22}+\tan \theta_{0} b_{32} \\
\frac{1}{\cos \theta_{0}} b_{31} & \frac{1}{\cos \theta_{0}} b_{32}
\end{array}\right],
$$


which has full rank. From (3.5.9), the first and second leading principal minors are

$$
\Delta_{1}=b_{21}+\tan \theta_{0} b_{31} \neq 0, \Delta_{2}=\operatorname{det}\left(K_{p}\right)=\frac{1}{\cos \theta_{0}}\left(b_{21} b_{32}-b_{22} b_{31}\right) \neq 0,
$$

since $b_{21}, b_{22}, b_{31}$, and $b_{32}$ are control gains from the corresponding control surfaces to the state accelerations and the signs are known: $b_{21}<0, b_{22}>0, b_{31}<0$, and $b_{32}<0$. Moreover, the equilibrium point of the pitch angle is $\theta_{0}=0.067 \mathrm{rad}$. Then, we obtain the sign information of the principal minors:

$$
\operatorname{sign}\left(\Delta_{1}\right)=-1, \quad \operatorname{sign}\left(\Delta_{2}\right)=1
$$

Reference model. The reference model is chosen as

$$
\Delta y_{m}(t)=W_{m}(s)[r](t)
$$

where $W_{m}(s)=\xi_{m}^{-1}(s)=\operatorname{diag}\left\{1 /(s+1)^{2}, 1 /(s+1)^{2}\right\}$.

Design parameters. Since the degree of $\xi_{m}(s)$ is 2, the filter is chosen as $h(s)=1 /(s+8)^{2}$. For the adaptive laws (3.3.14)-(3.3.16), we choose $\Gamma_{\theta 2}=10$, $\Gamma=\operatorname{diag}\{10,10\}$, and $D_{s}=\operatorname{diag}\left\{\operatorname{sign}\left[\Delta_{1}\right] \gamma_{1}, \operatorname{sign}\left[\frac{\Delta_{2}}{\Delta_{1}}\right] \gamma_{2}\right\}=\operatorname{diag}\{-30,-30\}$.

Adaptive state feedback controller. We apply the control law $\Delta u(t)=$ $K_{1}^{T}(t) \Delta x(t)+K_{2}(t) r(t)$ with $K_{1}(t)$ and $K_{2}(t)$ being updated from (3.3.14)-(3.3.16) to the linearized GTM first. Then, we apply $u(t)=u_{0}+\Delta u(t)$ to the nonlinear GTM around the equilibrium point $\left(x_{0}, u_{0}\right)$.

Simulations of linearized and nonlinear GTMs. In the simulation study, the initial values of the gain parameters $K_{1}(t)$ and $K_{2}(t)$ in the adaptive laws are chosen not far from the nominal values. We use two cases to assess the validation of our linearization-based control design:

(i) constant reference input $r(t)=r_{1}(t)=[4 \pi / 180,10 \pi / 180]^{T}$; 
(ii) varying reference input $r(t)=r_{2}(t)=[5 \pi / 180 \sin (0.02 t), 10 \pi / 180 \sin (0.015 t)]^{T}$. The simulation results of the linearized GTM and nonlinear GTM are given as follows.

Case I: $r(t)=r_{1}(t)$. In Figure 3.2, the dotted lines represent the reference $\Delta y_{m}(t)$, the dashed lines represent the output signal $\Delta y(t)$ of the linearized GTM (3.5.2), and the solid lines represent the GTM output perturbation $\Delta y(t)$. From Figure 3.2, we can see that the linearized GTM outputs track the reference signals, and the nonlinear GTM output perturbations track the reference signals as well. That is the output signal $y(t)$ of the nonlinear GTM can track the signal $y_{0}+\Delta y_{m}(t)$, which is not far from the equilibrium $y_{0}$.
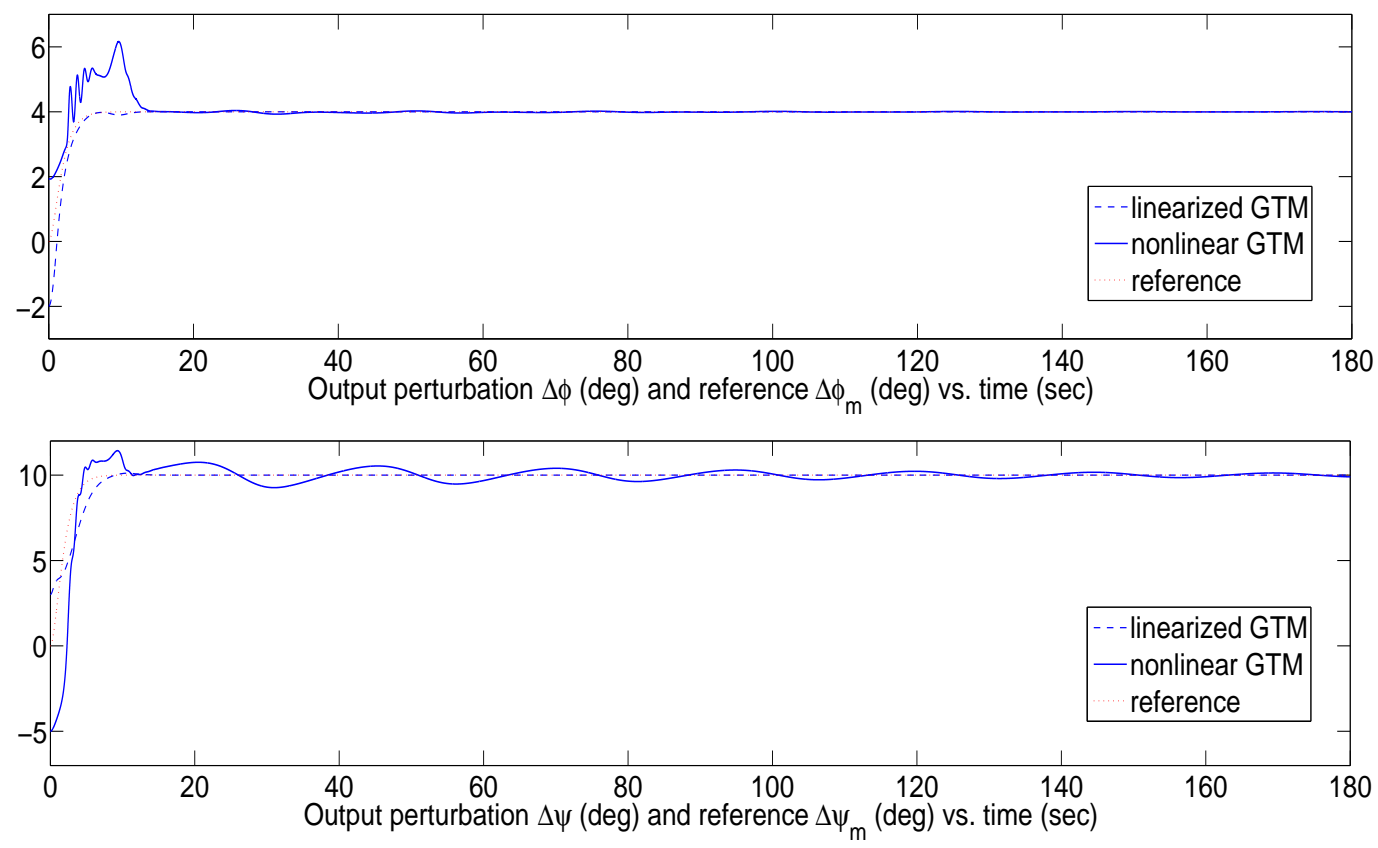

Figure 3.2: System responses vs. reference $\left(r(t)=r_{1}(t)\right)$.

Case II: $r(t)=r_{2}(t)$. From Figure 3.3, the output signals of the linearized and nonlinear GTMs can track given reference signals.

Summary of the simulation results. For the linearized GTM simulations in Case I and Case II, the output tracking is achieved, which is ensured by Theorem 

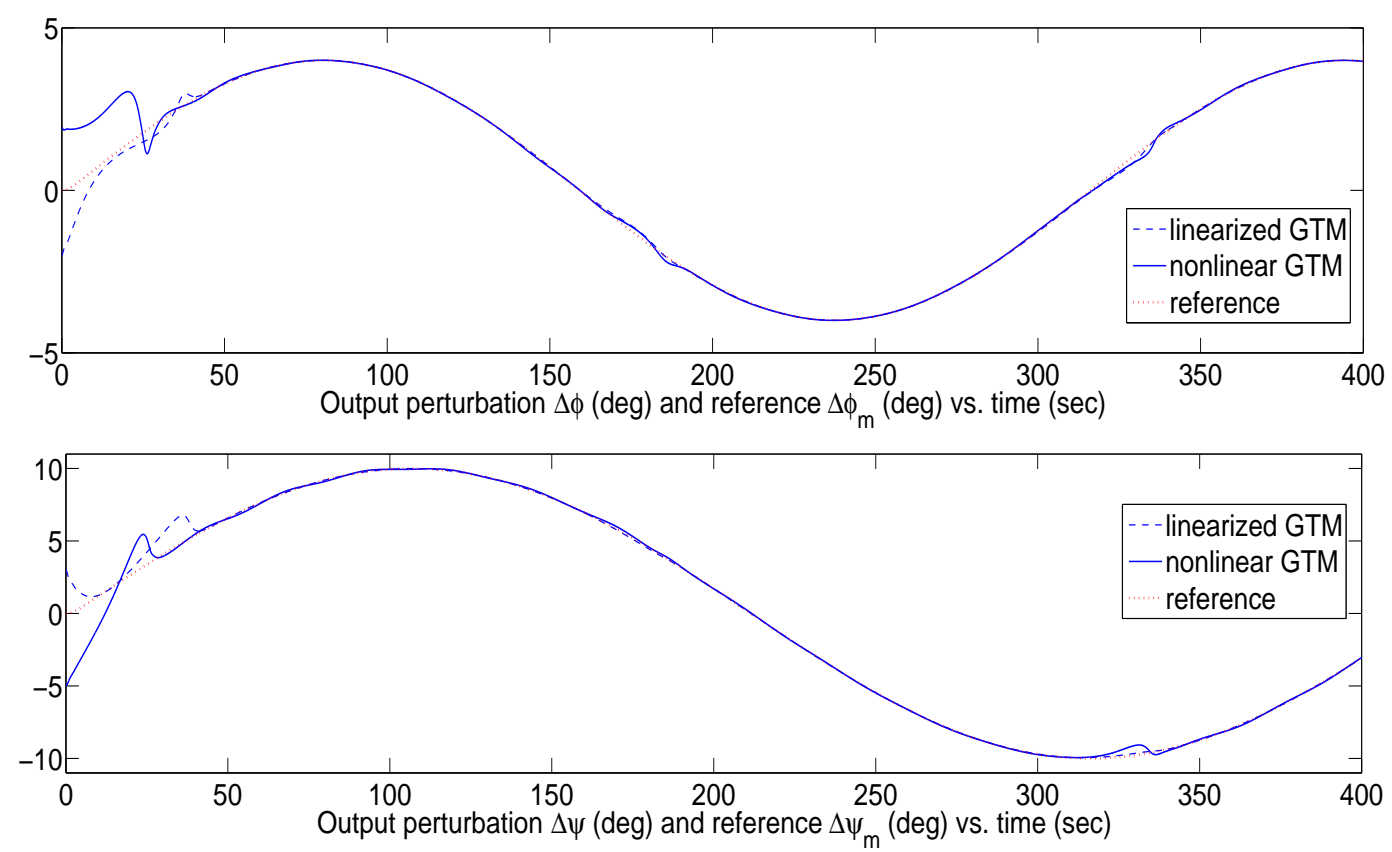

Figure 3.3: System responses vs. reference $\left(r(t)=r_{2}(t)\right)$.

3.3.1. From the nonlinear GTM simulation results in Case I and Case II, we conclude that this linearization-based control design can compensate the approximation errors and parameter uncertainties for the nonlinear system in a small neighborhood of the equilibrium point.

Applying fixed controller to the nonlinear GTM. To further show the advantage of the adaptive control scheme, we apply a linearization-based fixed controller $u(t)=u_{0}+\Delta u(t)$ to the nonlinear GTM, such a fixed controller $\Delta u(t)$ is chosen as the nominal controller $\Delta u(t)=K_{1}^{* T} \Delta x(t)+K_{2}^{*} r(t)$. The reference input is chosen as $r(t)=[4 \pi / 180,10 \pi / 180]^{T}$ the same with the one in Case I, where the adaptive controller achieves output tracking (Figure 3.2) for the nonlinear GTM. From Figure 3.4, we can see that the output tracking errors of the fixed control design are large. Thus, we may conclude that the linearization-based fixed controller may not be able to compensate the approximation error. 

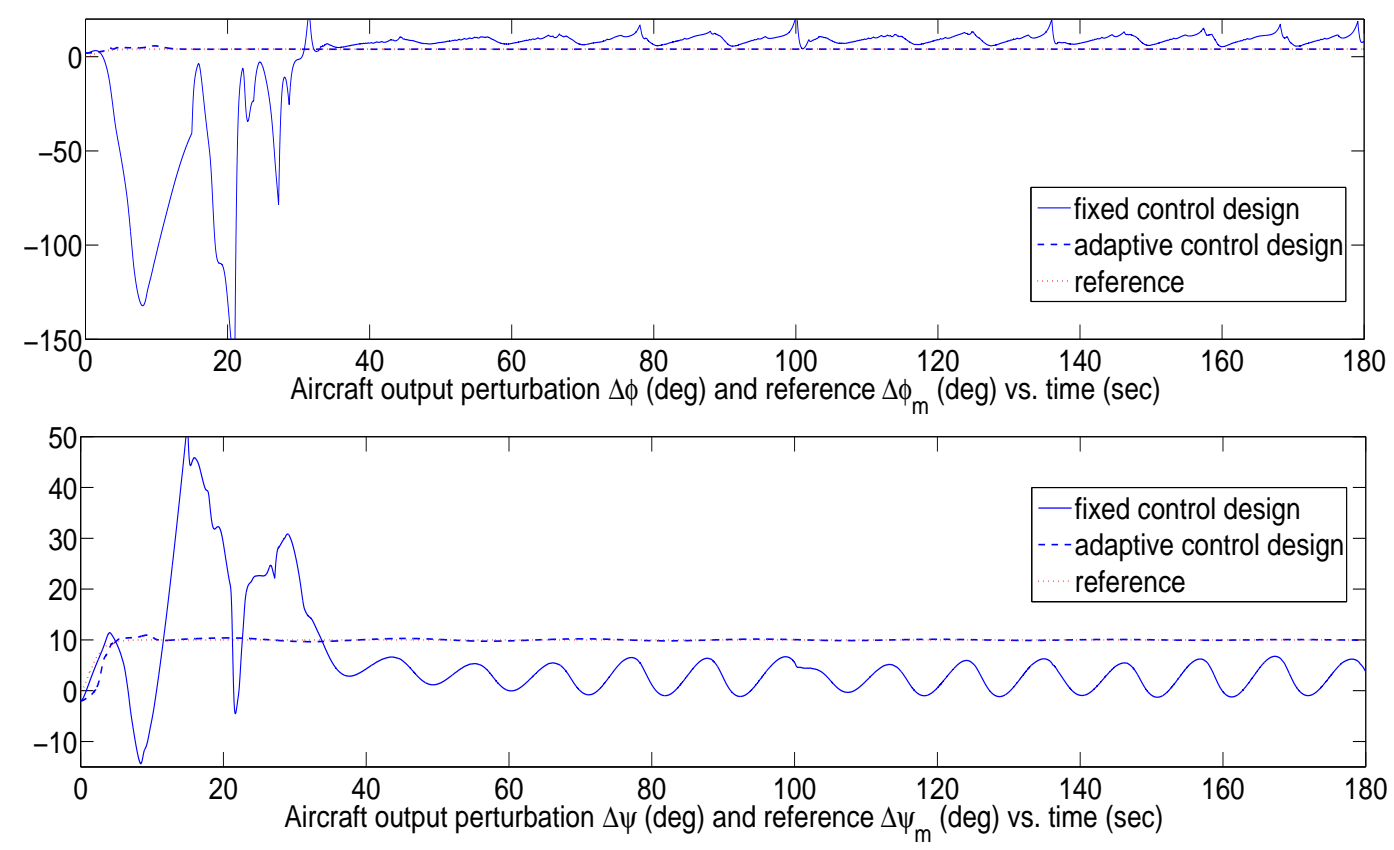

Figure 3.4: GTM outputs (adaptive and fixed) vs. reference.

\section{Summary}

In this chapter, we present the development of the state feedback output tracking multivariable MRAC scheme and the application to linear and nonlinear aircraft flight systems. Such schemes need less restrictive plant-model matching conditions than the state tracking scheme, while offering a simpler controller structure than the output feedback scheme. This state feedback for output tracking method represents an addition to the currently available collection of multivariable MRAC designs, and has a high potential for output tracking applications such as aircraft flight control in which system states are available but the state tracking matching conditions cannot be satisfied. The state feedback output tracking MRAC scheme design is based on the LDS decomposition of the plant's high frequency gain matrix, which needs much relaxed condition on $K_{p}$. Relaxed plant-model matching conditions and desired stability and 
asymptotic tracking properties have been established in theory and demonstrated via simulation results from application to a linearized GTM models. This linearizationbased design was subsequently applied to the nonlinear model of the GTM. The simulations have shown that such a linearization-based design is applicable to the nonlinear aircraft flight system in a small neighborhood of the equilibrium point. 


\section{Chapter 4}

\section{Adaptive Structural Damage Compensation for MIMO Systems}

This chapter develops a linearization-based adaptive control technique for control of the nonlinear aircraft flight systems with large parametric and structural uncertainties caused by damage. Some key technique issues are addressed:

- linearization of nonlinear aircraft systems with damage conditions;

- investigation of generic structures of linearized aircraft models; and

- invariance of infinite zero structures before and after damage.

Then, a state feedback multivariable model reference adaptive control (MRAC) scheme is developed to ensure stability and asymptotic output tracking for the aircraft flight system in the presence of damage. Simulation studies of this linearization-based adaptive control of the nonlinear aircraft system-the NASA GTM demonstrate the desired performance in a small neighborhood of the chosen operating point.

\subsection{Problem Statement}

The nonlinear aircraft flight system model constructed by force equations, moment equations, and kinematic equations, has been presented in Section 2.1, where the 
uncertain variations caused by damage are also investigated. We denote the nonlinear aircraft system model with two output signals pitch angle $\theta$ and yaw angle $\psi$ as

$$
\dot{x}(t)=f(x(t), u(t)), y(t)=C x(t)=[\theta, \psi]^{T},
$$

where $x(t)=\left[u_{b}, w_{b}, q_{b}, \theta, v_{b}, r_{b}, p_{b}, \phi, \psi\right]^{T}$ and $u(t)=\left[d_{e}, d_{r}, d_{a}, d_{t l}, d_{t r}\right]^{T}$. The first four state elements $u_{b}, w_{b}, q_{b}, \theta$ represent the longitudinal motion of the aircraft, while the last five elements $v_{b}, r_{b}, p_{b}, \phi, \psi$ represent the lateral-directional motion. To capture essential system structure features before and after damage occurs, we will analyze the linearized aircraft model at a given operating point $\left(x_{0}, u_{0}\right)$.

Operating point for linearization-based design. The operating point $\left(x_{0}, u_{0}\right)$ is important for the linearization-based design, since it connects the state, output, and control input signals between the nonlinear system and its linearized model in the following way:

$$
\Delta x(t)=x(t)-x_{0}, \Delta y(t)=y(t)-C x_{0}, \Delta u(t)=u(t)-u_{0}
$$

where $\Delta x(t), \Delta y(t)$, and $\Delta u(t)$ are the linearized perturbation state, output, and input signals, and $x(t), y(t)$, and $u(t)$ are the nonlinear aircraft system signals.

For the linearization-based feedback control design, the measurements of signals $\Delta x(t)$ or $\Delta y(t)$ are required. If $\left(x_{0}, u_{0}\right)$ is unknown, the signals $\Delta x(t)$ and $\Delta y(t)$ cannot be obtained from $x(t)$ and $y(t)$. Moreover, the designed linearization-based control law $\Delta u(t)$ cannot be applied to the nonlinear system, since $u_{0}$ is unknown in the nonlinear controller signal $u(t)=\Delta u(t)+u_{0}$. Therefore, to employ a linearizationbased design, the system should be linearized at a known operating point.

Damage causes uncertain system structure changes, equilibrium points are not accessible. In this research, a given point $\left(x_{0}, u_{0}\right)$ is chosen as the linearization operating point for the aircraft system (4.1.1) before and after damage occurs to ensure 
that the linearization-based design can be applied to the nonlinear aircraft system with damage. It is worth noting that the non-equilibrium operating point $\left(x_{0}, u_{0}\right)$ may introduce a dynamics offset term for the linearized system model.

Linearized aircraft system model. The linearized model of the nonlinear aircraft system $(4.1 .1)$ at $\left(x_{0}, u_{0}\right)$ is given as

$$
\Delta \dot{x}(t)=A \Delta x(t)+B \Delta u(t)+f_{0}, \quad \Delta y(t)=C \Delta x(t)
$$

where $f_{0}=f\left(x_{0}, u_{0}\right)$ may not be $0, A=\left.\frac{\partial f}{\partial x}\right|_{\left(x_{0}, u_{0}\right)}, B=\left.\frac{\partial f}{\partial u}\right|_{\left(x_{0}, u_{0}\right)}$, and

$$
C=\left[\begin{array}{lllllllll}
0 & 0 & 0 & 1 & 0 & 0 & 0 & 0 & 0 \\
0 & 0 & 0 & 0 & 0 & 0 & 0 & 0 & 1
\end{array}\right] \text {. }
$$

Since there exists uncertain damage, the system parameters $\left(A, B, f_{0}\right)$ are unknown and different before and after damage occurs. Assuming that the uncertain damage happens at $t=T_{d}$ with unknown $T_{d}$, it follows that

$$
\left(A, B, f_{0}\right)= \begin{cases}\left(A_{n}, B_{n}, f_{0 n}\right), & t \leq T_{d} \\ \left(A_{d}, B_{d}, f_{0 d}\right), & t>T_{d}\end{cases}
$$

where $\left(A_{n}, B_{n}, f_{0 n}\right)$ denotes the uncertain nominal system parameters and $\left(A_{d}, B_{d}, f_{0 d}\right)$ denotes the uncertain damaged system parameters.

Sequential linear system with dynamics offset. In this paper, a sequential linear system with an unknown constant dynamics offset is used to represent the linearized aircraft models before and after damage, such a system is described as

$$
\Delta \dot{x}(t)=A \Delta x(t)+B \Delta u(t)+f_{0}, \quad \Delta y(t)=C \Delta x(t)
$$

where $\Delta x(t) \in R^{M}, \Delta u(t) \in R^{M}$, and $\Delta y(t) \in R^{M}, A \in R^{n \times n}, B \in R^{n \times M}$, and $C \in R^{M \times n}$ are unknown piecewise constant matrices with a finite number of unknown jumps $\left(A_{i}, B_{i}, C_{i}\right), \quad i=1,2, \ldots, N$, and $f_{0} \in R^{n \times 1}$ is an unknown piecewise constant offset with a finite number of unknown jumps $f_{0 i}, \quad i=1,2, \ldots, N$, such that $A=$ 
$A_{i}, B=B_{i}, C=C_{i}, f_{0}=f_{0 i}$, for $t \in\left[t_{i-1}, t_{i}\right), i=1,2, \ldots, N$, with $t_{0}=0$ and $t_{N}=\infty$. This system description implies that there are up to $N-1$ damages which may occur in the system.

Control objective. The objective is to develop an adaptive control law $\Delta u(t)$ for (4.1.4) to make all the closed-loop system signals bounded and the system output signal $\Delta y(t)$ track a given reference signal $\Delta y_{m}(t)$ generated from a reference model:

$$
\Delta y_{m}(t)=W_{m}(s)[r](t), W_{m}(s)=\xi_{m}^{-1}(s)
$$

where $W_{m}(s)$ is a stable transfer matrix and $r(t)$ is a bounded reference input signal.

Control of the nonlinear aircraft system. It is worth noting that, to design an adaptive feedback control scheme for (4.1.4), the dimensions of control input $\Delta u(t) \in$ $R^{M}$ and system output $\Delta y(t) \in R^{M}$ should be the same. For the linearized aircraft system (4.1.2), the control input signal is

$$
\Delta u(t)=\left[\Delta d_{e}(t), \Delta d_{r}(t), \Delta d_{a}(t), \Delta d_{t l}(t), \Delta d_{t r}(t)\right]^{T} \in R^{5}
$$

while output signal is $\Delta y(t)=[\Delta \theta(t), \Delta \psi(t)]^{T} \in R^{2}$. In this research, we only manipulate the control input signal $\left[\Delta d_{e}(t), \Delta d_{r}(t)\right]^{T}$, while we set $\left[\Delta d_{a}, \Delta d_{t l}, \Delta d_{t r}\right]^{T}=0$. Therefore, the linearized aircraft system (4.1.2) becomes a 2-input and 2-output system with the control input signal $\Delta u(t)=\left[\Delta d_{e}(t), \Delta d_{r}(t)\right]^{T}$ and the output signal $\Delta y(t)=[\Delta \theta(t), \Delta \psi(t)]^{T}$. Then, after deriving the adaptive state feedback control scheme for $\Delta u(t)=\left[\Delta d_{e}(t), \Delta d_{r}(t)\right]^{T}$, the control law

$$
u(t)=\left[\Delta d_{e}(t)+d_{e 0}, \Delta d_{r}(t)+d_{r 0}, d_{a 0}, d_{t l 0}, d_{t r 0}\right]^{T},
$$

can be applied to the original nonlinear aircraft system (4.1.1) in a small neighborhood of the operating point $\left(x_{0}, u_{0}\right)$, where $u_{0}=\left[d_{e 0}, d_{r 0}, d_{a 0}, d_{t l 0}, d_{t r 0}\right]^{T}$ is the control input operating point. Figure 4.1 shows the closed-loop nonlinear aircraft system structure. 


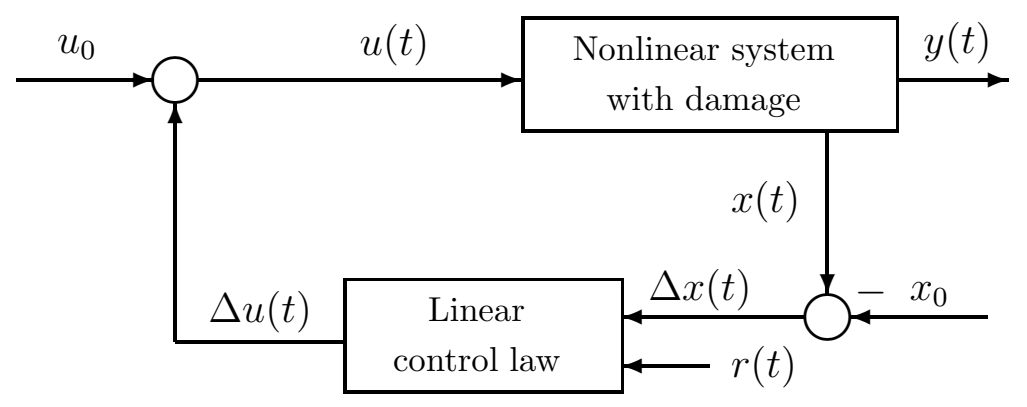

Figure 4.1: Closed-loop nonlinear aircraft control system with damage.

In the simulation studies, we will see that such a control law (4.1.7), with aileron $d_{a}(t)$ and throttles $d_{t l}(t), d_{t r}(t)$ fixed at the operating point values and elevator $d_{e}(t)$ and rudder $d_{r}(t)$ controlled by the linearization-based design, can efficiently achieve some longitudinal and lateral-directional maneuvers.

Assumptions. To design a multivariable state feedback MRAC scheme for the sequential linear system (4.1.4), the following assumptions are required: (A1) All zeros of $G_{i}(s)=C_{i}\left(s I-A_{i}\right)^{-1} B_{i}, i=1,2, \ldots, N$, have negative real parts; (A2) $G_{i}(s), i=1,2, \ldots, N$, have full rank, the modified left interactor matrix $\xi_{m}(s)$ is known and invariant for all $G_{i}(s), i=1,2, \ldots, N$; (A3) All leading principal minors $\Delta_{i j}, i=1,2, \ldots, N, j=1,2, \ldots, M$, of the high frequency gain matrices $K_{p i}=\lim _{s \rightarrow \infty} \xi_{m}(s) G_{i}(s)$ are nonzero and their signs are known, such that $\operatorname{sign}\left[\Delta_{p j}\right]=$ $\operatorname{sign}\left[\Delta_{q j}\right], p, q=1,2, \ldots, N, j=1,2, \ldots, M$, i.e. the signs are invariant when damage occurs; (A4) $\left(A_{i}, B_{i}\right)$ is controllable and $\left(A_{i}, C_{i}\right)$ is observable.

Assumptions (A1) and (A4) are needed for stable plant-model matching, assumption (A2) ensures that the aircraft systems can track a same reference system (4.1.5) before and after damage occurs, and assumption (A3) is used for designing adaptive parameter update laws. The invariance of interactor matrix (assumption (A2)) and signs of high frequency gain matrix (assumption (A3)) will be investigated for the linearized aircraft systems before and after damage occurs in the next section. 


\subsection{System Invariance of the Aircraft Model}

In this section, the linearized system invariance of infinite zero structure and signs of high frequency gain matrix before and after damage occurs will be studied from two directions: one is to investigate the generic structures of the linearized aircraft systems before and after damage; the other one is to investigate the relationship of the infinite zero structures between the nonlinear system and the linearized system to derive the invariant properties of linearized aircraft systems before and after damage.

Invariance analysis based on generic linearized models. The operating point is chosen as a wings-level flight condition

$$
\left(x_{0}, u_{0}\right)=\left(\left[u_{b 0}, w_{b 0}, 0, \theta_{0}, 0,0,0,0, \psi_{0}\right]^{T},\left[d_{e 0}, d_{r 0}, d_{a 0}, d_{t l 0}, d_{t r 0}\right]^{T}\right)
$$

After linearizing the aircraft system (4.1.1) at $\left(x_{0}, u_{0}\right)$ and setting the control input $\left[d_{a}(t), d_{t l}(t), d_{t r}(t)\right]^{T}=\left[d_{a 0}, d_{t l 0}, d_{t r 0}\right]^{T}$, the systems before and after damage are denoted as

$$
\Delta \dot{x}(t)=A \Delta x(t)+B \Delta u(t)+f_{0}, \quad \Delta y(t)=C \Delta x(t)
$$

where $\Delta u(t)=\left[\Delta d_{e}(t), \Delta d_{r}(t)\right]^{T}$ and

$$
C=\left[\begin{array}{lllllllll}
0 & 0 & 0 & 1 & 0 & 0 & 0 & 0 & 0 \\
0 & 0 & 0 & 0 & 0 & 0 & 0 & 0 & 1
\end{array}\right]
$$

When there is no damage, the longitudinal and lateral-directional dynamics are coupled, so that the parameters $A$ and $B$ are given as

$$
A=\left[\begin{array}{cc}
A_{1}^{(4 \times 4)} & 0^{(4 \times 5)} \\
0^{(5 \times 4)} & A_{4}^{(5 \times 5)}
\end{array}\right], \quad B=\left[\begin{array}{cc}
B_{1}^{(4 \times 1)} & 0^{(4 \times 1)} \\
0^{(5 \times 1)} & B_{4}^{(5 \times 1)}
\end{array}\right],
$$


where

$$
\begin{aligned}
& A_{1}=\left[\begin{array}{cccc}
a_{11} & a_{12} & a_{13} & a_{14} \\
a_{21} & a_{22} & a_{23} & a_{24} \\
a_{31} & a_{32} & a_{33} & a_{34} \\
0 & 0 & 1 & 0
\end{array}\right], A_{4}=\left[\begin{array}{ccccc}
a_{55} & a_{56} & a_{57} & a_{58} & 0 \\
a_{65} & a_{66} & a_{67} & a_{68} & 0 \\
a_{75} & a_{76} & a_{77} & a_{78} & 0 \\
0 & \tan \theta_{0} & 1 & 0 & 0 \\
0 & 1 / \cos \theta_{0} & 0 & 0 & 0
\end{array}\right], \\
& B_{1}=\left[\begin{array}{c}
b_{11} \\
b_{21} \\
b_{31} \\
0
\end{array}\right], B_{2}=\left[\begin{array}{c}
b_{62} \\
b_{72} \\
0 \\
0
\end{array}\right] .
\end{aligned}
$$

When damage occurs, the longitudinal and lateral-directional dynamics are coupled. The parameters $A$ and $B$ become to be

$$
A=\left[\begin{array}{ll}
A_{d 1}^{(4 \times 4)} & A_{d 2}^{(4 \times 5)} \\
A_{d 3}^{(5 \times 4)} & A_{d 4}^{(5 \times 5)}
\end{array}\right], \quad B=\left[\begin{array}{ll}
B_{d 1}^{(4 \times 1)} & B_{d 2}^{(4 \times 1)} \\
B_{d 3}^{(5 \times 1)} & B_{d 4}^{(5 \times 1)}
\end{array}\right],
$$

where

$$
\begin{aligned}
& A_{d 1}=\left[\begin{array}{cccc}
a_{d_{11}} & a_{d_{12}} & a_{d_{13}} & a_{d_{14}} \\
a_{d_{21}} & a_{d_{22}} & a_{d_{23}} & a_{d_{24}} \\
a_{d_{31}} & a_{d_{32}} & a_{d_{33}} & a_{d_{34}} \\
0 & 0 & 1 & 0
\end{array}\right], A_{d 2}=\left[\begin{array}{ccccc}
a_{d_{15}} & a_{d_{16}} & a_{d_{17}} & a_{d_{18}} & 0 \\
a_{d_{25}} & a_{d_{26}} & a_{d_{27}} & a_{d_{28}} & 0 \\
a_{d_{35}} & a_{d_{36}} & a_{d_{37}} & a_{d_{38}} & 0 \\
0 & 0 & 0 & 0 & 0
\end{array}\right], \\
& B_{d 1}=\left[\begin{array}{c}
b_{d_{11}} \\
b_{d_{21}} \\
b_{d_{31}} \\
0
\end{array}\right], B_{d 2}=\left[\begin{array}{c}
b_{d_{12}} \\
b_{d_{22}} \\
b_{d_{32}} \\
0
\end{array}\right] \\
& A_{d 3}=\left[\begin{array}{cccc}
a_{d_{51}} & a_{d_{52}} & a_{d_{53}} & a_{d_{54}} \\
a_{d_{61}} & a_{d_{62}} & a_{d_{63}} & a_{d_{64}} \\
a_{d_{71}} & a_{d_{72}} & a_{d_{73}} & a_{d_{74}} \\
0 & 0 & 0 & 0 \\
0 & 0 & 0 & 0
\end{array}\right], A_{d 4}=\left[\begin{array}{ccccc}
a_{d_{55}} & a_{d_{56}} & a_{d_{57}} & a_{d_{58}} & 0 \\
a_{d_{65}} & a_{d_{66}} & a_{d_{67}} & a_{d_{68}} & 0 \\
a_{d_{75}} & a_{d_{76}} & a_{d_{77}} & a_{d_{78}} & 0 \\
0 & a_{d_{86}} & 1 & 0 & 0 \\
0 & \frac{1}{\cos \theta_{0}} & 0 & 0 & 0
\end{array}\right], \\
& B_{d 3}=\left[\begin{array}{c}
b_{d_{51}} \\
b_{d_{61}} \\
b_{d_{71}} \\
0 \\
0
\end{array}\right], B_{d 4}=\left[\begin{array}{c}
b_{d_{52}} \\
b_{d_{62}} \\
b_{d_{72}} \\
0 \\
0
\end{array}\right]
\end{aligned}
$$


To determine the infinite zero structure for the system, relative degrees of entries of the transfer matrix $G(s)$ need to be investigated, which can be calculated as

$$
G(s)=\frac{1}{\operatorname{det}(s I-A)}\left(E_{n-1} s^{n-1}+E_{n-2} s^{n-2}+\cdots+E_{1} s+E_{0}\right),
$$

where $\operatorname{det}(s I-A) \triangleq s^{n}+\alpha_{n-1} s^{n-1}+\cdots+\alpha_{1} s+\alpha_{0}, E_{n-1}=C B, E_{n-2}=C A B+$ $\alpha_{n-1} C B, \ldots$, and $E_{0}=C A^{n-1} B+\alpha_{n-1} C A^{n-2} B+\cdots+\alpha_{1} C B$.

For the nominal aircraft system without damage, the parameters $A$ and $B$ are given as (4.2.4) with the matrix $C$ given as (4.2.3), so the coefficients $E_{n-1}$ and $E_{n-2}$ for $G(s)$ are given as

$$
E_{n-1}=C B=0, \quad E_{n-2}=C A B=\operatorname{diag}\left\{b_{31}, \frac{b_{61}}{\cos \theta_{0}}\right\}
$$

Based on Lemma 2.3.1, the interactor matrix of $G(s)$ can be chosen as $\xi_{m}(s)=$ $\operatorname{diag}\left\{(s+1)^{2},(s+1)^{2}\right\}$, it follows that the high frequency gain matrix is $K_{p}=$ $\lim _{s \rightarrow \infty} \xi_{m}(s) G(s)=C A B=\operatorname{diag}\left\{b_{31}, \frac{b_{61}}{\cos \theta_{0}}\right\}$. Since the parameters $b_{31}$ and $b_{61}$ are the control gains from elevator to pitch acceleration and rudder to yaw acceleration, the signs of these parameters can be obtained: $b_{31}<0$ and $b_{61}<0$. So, the signs of the leading principal minors are $\operatorname{sign}\left(\Delta_{1}\right)=\operatorname{sign}\left(b_{31}\right)=-1, \operatorname{sign}\left(\Delta_{2}\right)=\operatorname{sign}\left(\frac{b_{31} b_{61}}{\cos \theta_{0}}\right)=1$.

After damage occurs, the matrices $A$ and $B$ change to the damaged values given as (4.2.5). So the coefficients $E_{n-1}$ and $E_{n-2}$ are calculated as

$$
E_{n-1}=C B=0, \quad E_{n-2}=C A B=\left[\begin{array}{cc}
b_{d_{31}} & b_{d_{32}} \\
\frac{1}{\cos \theta_{0}} b_{d_{61}} & \frac{1}{\cos \theta_{0}} b_{d_{62}}
\end{array}\right]
$$

The interactor matrix of $G(s)$ can be chosen as $\xi_{m}(s)=\operatorname{diag}\left\{(s+1)^{2},(s+1)^{2}\right\}$, and the high frequency gain matrix $K_{p}=\lim _{s \rightarrow \infty} \xi_{m}(s) G(s)=C A B$. If the shift of center of gravity is small, the signs of $b_{d_{31}}$ and $b_{d_{62}}$ may still be negative, and the coupling terms may be very small. That is $\operatorname{sign}\left(\Delta_{1}\right)=-1, \operatorname{sign}\left(\Delta_{2}\right)=1$.

From the above generic structure analysis, it follows that the infinite zero structure is invariant before and after damage, which is $\xi_{m}(s)=\operatorname{diag}\left\{(s+1)^{2},(s+1)^{2}\right\}$, and 
the signs of the leading principal minors of high frequency matrix are invariant when the shift of center of gravity is small, which $\operatorname{are} \operatorname{sign}\left(\Delta_{1}\right)=-1$ and $\operatorname{sign}\left(\Delta_{2}\right)=1$. Similar generic invariance analysis for the linearized aircraft systems with different output signals and control input signals can be found in [57].

Invariance analysis of linearization. In this part, the relationship between interactor matrix $\xi_{m}(s)$ of the linearized system and relative degree of the nonlinear system will be studied. The nonlinear aircraft model (4.1.1) before and after damage can be denoted as

$$
\dot{x}(t)=f(x(t))+\sum_{i=1}^{2} g_{i}(x(t)) u_{i}(t), \quad y(t)=\left[h_{1}(x(t)), h_{2}(x(t))\right]^{T}=C x(t)=[\theta, \phi]^{T},
$$

where $u_{1}=d_{e}, u_{2}=d_{r}$, and the other control input signals $\left[d_{a}, d_{t l}, d_{t r}\right]^{T}=\left[d_{a 0}, d_{t l 0}, d_{t r 0}\right]^{T}$.

The nonlinear aircraft model (4.2.7) has a set of relative degrees $\left\{\rho_{1}, \rho_{2}\right\}$ defined at the operating point $\left(x_{0}, u_{0}\right)$, such that (i) $L_{g_{j}} L_{f}^{k} h_{i}(x)=0, i=1,2, j=1,2, k<\rho_{i}-1$, for $\forall x$ in the neighborhood of $x_{0}$, and (ii) the matrix

$$
\alpha(x)=\left[\begin{array}{cc}
L_{g_{1}} L_{f}^{\rho_{1}-1} h_{1}(x) & L_{g_{M}} L_{f}^{\rho_{1}-1} h_{1}(x) \\
L_{g_{1}} L_{f}^{\rho_{2}-1} h_{2}(x) & L_{g_{M}} L_{f}^{\rho_{2}-1} h_{2}(x)
\end{array}\right]
$$

is nonsingular at $x=x_{0}[43,84]$. The linearized system at $\left(x_{0}, u_{0}\right)$ is given as

$$
\Delta \dot{x}(t)=\left(f\left(x_{0}\right)+\sum_{i=1}^{2} g_{i}\left(x_{0}\right) u_{i 0}\right)+A \Delta x(t)+B \Delta u(t), \quad \Delta y(t)=C \Delta x(t),
$$

where $\Delta x(t)=x(t)-x_{0}, \Delta u(t)=u(t)-u_{0}, \Delta y(t)=y(t)-C x_{0}$, and

$$
\begin{aligned}
A & =\bar{A}+\Delta A, \quad \bar{A}=\left.\frac{\partial f}{\partial x}\right|_{x=x_{0}}, \Delta A=\left.\frac{\partial g_{1}}{\partial x}\right|_{x=x_{0}} u_{10}+\left.\frac{\partial g_{2}}{\partial x}\right|_{x=x_{0}} u_{20}, \\
B & =\left[g_{1}\left(x_{0}\right), g_{2}\left(x_{0}\right)\right] .
\end{aligned}
$$

From the definition of the nonlinear system relative degree $\left\{\rho_{1}, \rho_{2}\right\}$ in (4.2.8) and the matrices $A$ and $B$, and $C$ in (4.2.9), the following results can be established to show 
the connections between the interactor matrix of the linearized system $\xi_{m}(s)$ and the nonlinear system relative degree $\left\{\rho_{1}, \rho_{2}\right\}$ : (i) The interactor matrix of the linearized system (4.2.9) is of diagonal form: $\xi_{m}(s)=\operatorname{diag}\left\{s+a_{1}, s+a_{2}\right\}$, where $a_{1}>0, a_{2}>0$, if and only if the relative degree of the nonlinear system is $\left\{\rho_{1}, \rho_{2}\right\}=\{1,1\}$; (ii) If the relative degree of the nonlinear system is $\left\{\rho_{1}, \rho_{2}\right\}=\{1,2\}$, then the interactor matrix of the linearized system (4.2.9) is of diagonal form: $\xi_{m}(s)=\operatorname{diag}\left\{s+a,(s+a)^{2}\right\}$, where $a>0$; (iii) If the relative degree of the nonlinear system is $\left\{\rho_{1}, \rho_{2}\right\}=\{2,2\}$, then the interactor matrix of the linearized system (4.2.9) is of diagonal form: $\xi_{m}(s)=$ $\operatorname{diag}\left\{(s+a)^{2},(s+a)^{2}\right\}$, where $a>0$.

For higher relative degree case, similar results may also be derived. The above results build the connections between the interactor matrix $\xi_{m}(s)$ and the nonlinear system relative degree. In the multivariable MRAC design, the invariance property of $\xi_{m}(s)$ before and after damage is a crucial condition. To determine the invariance of $\xi_{m}(s)$ before and after damage, the relative degrees of the nonlinear aircraft model before and after damage can be studied. Assuming that the relative degree $\left\{\rho_{1}, \rho_{2}\right\}$ of the nonlinear aircraft model at the operating point $\left(x_{0}, u_{0}\right)$ is invariant before and after damage, the linearized systems before and after damage at such an operating point $\left(x_{0}, u_{0}\right)$ have the same interactor matrix $\xi_{m}(s)$.

Based on the above analysis, the design conditions: the invariance of infinite zero structure (assumption (A2)) and the invariance of signs of leading principal minors (assumption (A3)) can be satisfied for the aircraft system. In the next section, a state feedback for output tracking MRAC scheme will be developed. 


\subsection{The Multivariable MRAC Scheme}

In this section, the multivariable state feedback model reference adaptive control design for the sequential linearized system (4.1.4) will be developed to accommodate the unknown dynamics offset $f_{0}$ and the uncertainties of system parameters $(A, B, C)$.

State feedback controller design. To compensate the constant offset term $f_{0}$ in (4.1.4), the state feedback controller structure is chosen as

$$
\Delta u(t)=K_{1}^{T}(t) \Delta x(t)+K_{2}(t) r(t)+k_{3}(t)
$$

where $k_{3}(t) \in R^{M}$ is the adaptive estimate of an unknown constant compensation term $k_{3}^{*}$ (to be derived next) for canceling the effect of the constant offset $f_{0}$, and $K_{1}(t)$ and $K_{2}(t)$ are the estimates of the nominal piecewise constant parameters $K_{1}^{*}$ and $K_{2}^{*}$ which satisfy the plant-model matching condition (2.4.3):

$$
C\left(s I-A-B K_{1}^{* T}\right)^{-1} B K_{2}^{*}=W_{m}(s), K_{2}^{*-1}=K_{p},
$$

for each damage scenarios, where $K_{p}$ is a piecewise constant, for each jump, $K_{p}=$ $K_{p i}=\lim _{s \rightarrow \infty} \xi_{m}(s) G_{i}(s), i=1,2, \ldots, N$.

To derive the nominal value $k_{3}^{*}$, the nominal controller

$$
\Delta u(t)=K_{1}^{* T} \Delta x(t)+K_{2}^{*} r(t)+k_{3}^{*}
$$

is applied to the system (4.1.4) to achieve exact plant-model matching. Consider a particular set of constant values of the system parameters $\left(A, B, f_{0}\right)$. Then, substituting (4.3.3) in the system (4.1.4), the closed-loop system in the frequency $s$-domain is given as

$$
\Delta y(s)=C\left(s I-A-B K_{1}^{* T}\right)^{-1} B K_{2}^{*} r(s)+\Delta(s),
$$

where

$$
\Delta(s)=C\left(s I-A-B K_{1}^{* T}\right)^{-1}\left(B \frac{k_{3}^{*}}{s}+\frac{f_{0}}{s}\right)
$$


From the reference system (4.1.5) and the matching conditions (4.3.2), the output tracking error in the frequency $s$-domain is

$$
e(s)=\Delta y(s)-\Delta y_{m}(s)=\Delta(s) .
$$

Applying the Laplace final value theorem, it has

$$
\lim _{t \rightarrow \infty} e(t)=\lim _{s \rightarrow 0} s \Delta(s)=D k_{3}^{*}+d
$$

where $D=-C\left(A+B K_{1}^{*}\right)^{-1} B$ and $d=-C\left(A+B K_{1}^{*}\right)^{-1} f_{0}$. For offset rejection, $k_{3}^{*}$ is given as

$$
k_{3}^{*}=-D^{-1} d,
$$

and then from (4.3.7)-(4.3.8) with $\delta(t)=\mathcal{L}^{-1}[\Delta(s)]$, it follows that

$$
\lim _{t \rightarrow \infty}\left(\Delta y(t)-\Delta y_{m}(t)\right)=\lim _{t \rightarrow \infty} \delta(t)=0 .
$$

Since the system parameters $\left(A, B, f_{0}\right)$ are piecewise constant, the nominal matching parameter $k_{3}^{*}$ is also piecewise constant, as defined above for each set of $\left(A, B, f_{0}\right)$.

Robustness of matching conditions. For the assumption (A2) (which holds for the aircraft flight systems before and after damage occurs), all the uncertain systems $\left(A_{i}, B_{i}, C_{i}\right), i=1,2, \ldots, N$, have the same interactor matrix $\xi_{m}(s)$. Then, based on the matching conditions (4.3.2) and (4.3.8), it follows that there exists a nominal controller (4.3.3) to make the output signals of all the systems $\left(A_{i}, B_{i}, C_{i}\right), i=$ $1,2, \ldots, N$, track some reference signals generated from a common reference system $W_{m}(s)=\xi_{m}^{-1}(s)$.

Tracking error equation. Substituting the control law (4.3.1) in the system (4.1.4), the system dynamics is given as

$$
\begin{aligned}
\Delta \dot{x}(t) & =\left(A+B K_{1}^{* T}\right) \Delta x(t)+B K_{2}^{*} r(t)+B k_{3}^{*}+f_{0}+B\left(\widetilde{K}_{1}^{T}(t) \Delta x(t)+\widetilde{K}_{2}(t) r(t)+\tilde{k}_{3}(t)\right) \\
\Delta y(t) & =C \Delta x(t),
\end{aligned}
$$


where $\widetilde{K}_{1}^{T}(t)=K_{1}^{T}(t)-K_{1}^{*}, \widetilde{K}_{2}(t)=K_{2}(t)-K_{2}^{*}$, and $\tilde{k}_{3}(t)=k_{3}(t)-k_{3}^{*}$. In view of the reference model (4.1.5), matching conditions (4.3.2), (4.3.8), and (4.3.10), the output tracking error is given as

$$
e(t)=\Delta y(t)-\Delta y_{m}(t)=W_{m}(s) K_{p}\left[\widetilde{\Theta}^{T} \omega\right](t)+\delta(t),
$$

where $\widetilde{\Theta}(t)=\Theta(t)-\Theta^{*}, \Theta(t)=\left[K_{1}^{T}(t), K_{2}(t), k_{3}(t)\right]^{T}, \Theta^{*}=\left[K_{1}^{* T}, K_{2}^{*}, k_{3}^{*}\right]^{T}, \omega(t)=$ $\left[\Delta x^{T}(t), r^{T}(t), 1\right]^{T}$. To deal with the uncertainty of $K_{p}$, the LDS decomposition 2.3.5

$$
K_{p}=L_{s} D_{s} S
$$

is used, where $S \in R^{M \times M}$ with $S=S^{T}>0, L_{s}$ is an $M \times M$ unit lower triangular matrix, and

$$
D_{s}=\operatorname{diag}\left\{s_{1}^{*}, s_{2}^{*}, \ldots, s_{M}^{*}\right\}=\operatorname{diag}\left\{\operatorname{sign}\left[\Delta_{1}\right] \gamma_{1}, \ldots, \operatorname{sign}\left[\frac{\Delta_{M}}{\Delta_{M-1}}\right] \gamma_{M}\right\}
$$

such that $\gamma_{i}>0, i=1, \ldots, M$, may be arbitrarily chosen.

Remark 4.3.1. In the adaptive law design, $D_{s}$ matrix will be used as a gain matrix. Although $K_{p}$ is a piecewise constant, based on the Assumption (A3), a uniform $D_{s}$ can be chosen for all the high frequency gain matrices to derive the adaptive laws.

Tracking error parametrization. To obtain the adaptive laws for $K_{1}(t), K_{2}(t)$, and $k_{3}(t)$, a well parameterized tracking error model is needed. Substituting the LDS decomposition (4.3.12) of $K_{p}$ (with a uniform $D_{s}$ ) in (4.3.11), and ignoring the exponentially decaying term $\delta(t)$, the error equation can be parameterized as

$$
L_{s}^{-1} \xi_{m}(s)[e](t)=D_{s} S \tilde{\Theta}^{T}(t) \omega(t) .
$$

Since $\xi_{m}(s)$ is not proper, the signal $\xi_{m}(s)[e](t)$ is not accessible. So, a filter $h(s)=$ $1 / f_{h}(s)$, where $f_{h}(s)$ is a stable and monic polynomial of degree equals to the degree 
of $\xi_{m}(s)$, is introduced. Operating both sides of (4.3.14) by $h(s) I_{M}$ leads to

$$
\bar{e}(t)+\left[0, \theta_{2}^{* T} \eta_{2}(t), \theta_{3}^{* T} \eta_{3}(t), \ldots, \theta_{M}^{* T} \eta_{M}(t)\right]^{T}=D_{s} S h(s)\left[\tilde{\Theta}^{T} \omega\right](t)
$$

where $\Theta_{0}^{*}=L_{s}^{-1}-I=\left\{\theta_{i j}^{*}\right\}$ with $\theta_{i j}^{*}=0$ for $i=1,2, \ldots, M, j \geq i, \bar{e}(t)=$ $\xi_{m}(s) h(s)[e](t)=\left[\bar{e}_{1}(t), \ldots, \bar{e}_{M}(t)\right]^{T}, \eta_{i}(t)=\left[\bar{e}_{1}, \ldots, \bar{e}_{i-1}\right]^{T}$, and $\theta_{i}^{*}=\left[\theta_{i 1}^{*}, \ldots, \theta_{i i-1}^{*}\right]^{T}$, $i=2, \ldots, M$. It is worth noting that $\bar{e}(t)$ can be obtained from $e(t)$, since $\xi_{m}(s) h(s)$ is strictly proper. In (4.3.15), there are unknown parameters $D_{s} S$ and $\theta_{i}^{*}, i=2, \ldots, M$, which are still needed to be estimated. So, an estimation error model containing estimates of $D_{s} S$ and $\theta_{i}^{*}, i=2, \ldots, M$, is established.

Estimation error. Introduce an estimation error signal as

$$
\epsilon(t)=\left[0, \theta_{2}^{T}(t) \eta_{2}(t), \theta_{3}^{T}(t) \eta_{3}(t), \ldots, \theta_{M}^{T}(t) \eta_{M}(t)\right]^{T}+\Psi(t) \xi(t)+\bar{e}(t)
$$

where $\theta_{i}(t), i=2,3, \ldots, M$, are the estimates of $\theta_{i}^{*}$, and $\Psi(t)$ is the estimate of $\Psi^{*}=D_{s} S$, and

$$
\xi(t)=\Theta^{T}(t) \zeta(t)-h(s)\left[\Theta^{T} \omega\right](t), \zeta(t)=h(s)[\omega](t)
$$

From (4.3.15) and (4.3.16), the estimation error $\epsilon(t)$ can be parameterized as

$$
\epsilon(t)=\left[0, \tilde{\theta}_{2}^{T}(t) \eta_{2}(t), \tilde{\theta}_{3}^{T}(t) \eta_{3}(t), \ldots, \tilde{\theta}_{M}^{T}(t) \eta_{M}(t)\right]^{T}+D_{s} S \tilde{\Theta}^{T}(t) \zeta(t)+\tilde{\Psi}(t) \xi(t)
$$

where $\tilde{\theta}_{i}(t)=\theta_{i}(t)-\theta_{i}^{*}, i=2,3, \ldots, M$, and $\tilde{\Psi}(t)=\Psi(t)-\Psi^{*}$ are the related parameter errors.

Adaptive laws. With the estimation error model (4.3.18), adaptive laws are chosen as

$$
\begin{aligned}
\dot{\theta}_{i}(t) & =-\frac{\Gamma_{\theta i} \epsilon_{i}(t) \eta_{i}(t)}{m^{2}(t)}, i=2,3, \ldots, M \\
\dot{\Theta}^{T}(t) & =-\frac{D_{s} \epsilon(t) \zeta^{T}(t)}{m^{2}(t)} \\
\dot{\Psi}(t) & =-\frac{\Gamma \epsilon(t) \xi^{T}(t)}{m^{2}(t)}
\end{aligned}
$$


where the signal $\epsilon(t)=\left[\epsilon_{1}(t), \epsilon_{2}(t), \ldots, \epsilon_{M}(t)\right]^{T}$ is computed from (4.3.16), $\Gamma_{\theta i}=\Gamma_{\theta i}^{T}>$ $0, i=2,3, \ldots, M$, and $\Gamma=\Gamma^{T}>0$ are adaptation gain matrices, and

$$
m(t)=\sqrt{1+\zeta^{T}(t) \zeta(t)+\xi^{T}(t) \xi(t)+\sum_{i=2}^{M} \eta_{i}^{T}(t) \eta_{i}(t)}
$$

is a standard normalization signal.

Stability analysis. From the adaptive laws (4.3.19)-(4.3.21), the following stability properties can be derived.

Lemma 4.3.1. The adaptive laws (4.3.19)-(4.3.21) ensure that

(i) $\theta_{i}(t) \in L^{\infty}, i=2,3, \ldots, M, \Theta(t) \in L^{\infty}, \Psi(t) \in L^{\infty}$, and $\frac{\epsilon(t)}{m(t)} \in L^{2} \cap L^{\infty}$;

(ii) $\dot{\theta}_{i}(t) \in L^{2} \cap L^{\infty}, i=2,3, \ldots, M, \dot{\Theta}(t) \in L^{2} \cap L^{\infty}$, and $\dot{\Psi}(t) \in L^{2} \cap L^{\infty}$.

Proof: Consider the positive definite function

$$
V=\frac{1}{2}\left(\sum_{i=2}^{m} \tilde{\theta}_{i}^{T} \Gamma_{\theta i}^{-1} \tilde{\theta}_{i}+\operatorname{tr}\left[\tilde{\Psi}^{T} \Gamma^{-1} \tilde{\Psi}\right]+\operatorname{tr}\left[\tilde{\Theta} S \tilde{\Theta}^{T}\right]\right),
$$

which is continuous over each interval $\left(t_{i-1}, t_{i}\right), i=1,2, \ldots, N$, with $t_{0}=0$ and $t_{N}=\infty$, and has a finite jump at $t_{i}, i=1,2, \ldots, N-1$, i.e.,

$$
V\left(t_{i}^{+}\right)-V\left(t_{i}^{-}\right)<\infty, i=1,2, \ldots, N-1 .
$$

From the adaptive laws (4.3.19)-(4.3.21), the time-derivative of $V$ in each $\left(t_{i-1}, t_{i}\right)$ is obtained as

$$
\dot{V}=-\sum_{i=2}^{M} \frac{\tilde{\theta}_{i}^{T} \epsilon_{i}(t) \eta_{i}(t)}{m^{2}(t)}-\frac{\xi^{T}(t) \tilde{\Psi}^{T} \epsilon(t)}{m^{2}(t)}-\frac{\zeta^{T}(t) \tilde{\Theta} S D_{s} \epsilon(t)}{m^{2}(t)}=-\frac{\epsilon^{T}(t) \epsilon(t)}{m^{2}(t)} \leq 0 .
$$

This means that $V\left(t_{i}^{-}\right) \leq V\left(t_{i-1}^{+}\right)$. From (4.3.24) and (4.3.25), $V(t)$ is bounded for $[0, \infty)$, so that $\theta_{i}(t) \in L^{\infty}, i=2,3, \ldots, M, \Theta(t) \in L^{\infty}$, and $\Psi(t) \in L^{\infty}$. Then, the integration of both sides of (4.3.25) is given as

$$
\int_{0}^{\infty} \frac{\epsilon^{T}(\tau) \epsilon(\tau)}{m^{2}(\tau)} d \tau=V(0)+\sum_{i=1}^{N-1}\left[V\left(t_{i}^{+}\right)-V\left(t_{i}^{-}\right)\right]-V(\infty)
$$


Since $V$ is bounded for $[0, \infty)$, it follows that

$$
\int_{0}^{\infty} \frac{\epsilon^{T}(\tau) \epsilon(\tau)}{m^{2}(\tau)} d \tau<\infty
$$

from which $\frac{\epsilon(t)}{m(t)} \in L^{2} \cap L^{\infty}$ can be concluded. Since the normalized signals $\frac{\eta_{i}(t)}{m(t)}, \frac{\zeta^{T}(t)}{m(t)}$, $\frac{\xi^{T}(t)}{m(t)} \in L^{\infty}$, it can be concluded that $\dot{\theta}_{i}(t) \in L^{2} \cap L^{\infty}, i=2,3, \ldots, M, \dot{\Theta}(t) \in L^{2} \cap L^{\infty}$, and $\dot{\Psi}(t) \in L^{2} \cap L^{\infty}$.

Based on these properties, the desired closed-loop system properties can be proved: Theorem 4.3.1. The multivariable MRAC scheme with the state feedback control law (4.3.1) updated by the adaptive laws (4.3.19)-(4.3.21), when applied to the system (4.1.4), guarantees the closed-loop signal boundedness and asymptotic output tracking: $\lim _{t \rightarrow \infty}\left(\Delta y(t)-\Delta y_{m}(t)\right)=0$, for any initial conditions.

The proof of Theorem 4.3.1 can be carried out by using a similar way as described in [46] for multivariable MRAC using output feedback. A key step of such an analysis procedure is to express a filtered version of the plant output $\Delta y(t)$ in a feedback framework which has a small gain due to the $L^{2}$ properties of $\dot{\Theta}(t), \dot{\theta}_{i}(t)$ and $\frac{\epsilon(t)}{m(t)}$. The state feedback control signal $\Delta u(t)$ is required to be expressed in terms of the output $\Delta y(t)$. This can be done using a state observer representation of the plant $\Delta \dot{x}(t)=A \Delta x(t)+B \Delta u(t)+f_{0}, \Delta y(t)=C \Delta x(t): \Delta \dot{x}(t)=(A-L C) \Delta x(t)+$ $B \Delta u(t)+L \Delta y(t)+f_{0}$ for a gain matrix $L \in R^{n \times M}$ such that $A-L C$ is stable. Then, the analysis procedure in [46] can be used to conclude the closed-loop signal boundedness and output tracking: $\lim _{t \rightarrow \infty}\left(\Delta y(t)-\Delta y_{m}(t)\right)=0$.

\subsection{Simulation Study for the GTM}

In this section, the developed linearization-based adaptive control design is applied to the NASA generic transport model (GTM) with damage to assess its effectiveness for the nonlinear aircraft system. 
Damage scenario. The GTM model contains several damage scenarios. In this study, the damage case with loss of outboard left wing-tip is selected, which is approximate $25 \%$ semi-span of the left wing.

\subsubsection{Control of the Linearized GTM Model}

The nominal and damaged GTMs are linearized at an operating condition (wingslevel flight at 90 knots) $\left(x_{0}, u_{0}\right)$, where $x_{0}=\left[u_{b 0}, w_{b 0}, 0, \theta_{0}, 0,0,0,0, \psi_{0}\right]^{T}$, to ensure the invariance of the infinite zero structure and the signs of principal minors of the high frequency gain matrices. The linearized piecewise system is described as

$$
\Delta \dot{x}(t)=A \Delta x(t)+B \Delta u(t)+f_{0}, \Delta y(t)=C \Delta x(t) .
$$

The numerical parameter values used for building the aircraft simulation models and verifying the design assumptions are given as follows. When there is no damage, $A=A_{1}, B=B_{1}$, and $f_{0}=0$, after the loss of wing-tip damage occurs, $A=A_{2}$, $B=B_{2}$, and $f_{0}=[0.04,-0.93,-0.09,0,0.21,0,0.48,0.02,0]^{T}$, where

$$
\begin{gathered}
A_{1}=\left[\begin{array}{ccccccccc}
-0.0380 & 0.2807 & -7.4780 & -32.10 & -0.0037 & -0.0005 & 0 & 0 & 0 \\
-0.2460 & -3.4110 & 146.2 & -2.1750 & 0.0004 & 0 & 0.0005 & -0.0069 & 0 \\
0.0130 & -0.3579 & -4.4020 & 0 & 0.0085 & 0.0377 & -0.0016 & 0 & 0 \\
0 & 0 & 1 & 0 & 0 & -0.0002 & 0 & 0.0002 & 0 \\
-0.0004 & 0.0017 & 0 & -0.0005 & -0.6868 & -151.5 & 7.9150 & 32.10 & 0 \\
0 & -0.0009 & -0.0293 & 0 & 0.2735 & -1.6850 & -0.2679 & 0 & 0 \\
0 & -0.0001 & 0.0020 & 0 & -0.7289 & 1.8910 & -6.7690 & 0 & 0 \\
0 & 0 & 0 & -0.0002 & 0 & 0.0678 & 1 & 0 & 0 \\
0 & 0 & 0.0002 & 0 & 0 & 1.0020 & 0 & 0.0002 & 0
\end{array}\right], \\
A_{2}=\left[\begin{array}{cccccccccc}
-0.0361 & 0.1460 & -8.3640 & -32.09 & 0.0099 & -0.2118 & 0 & 0 & 0 \\
-0.1531 & -3.1150 & 146 & -2.337 & 0.5486 & 0 & 0.2118 & 0.7877 & 0 \\
0.0162 & -0.1789 & -4.2230 & 0 & -0.0046 & -0.4568 & 0.1079 & 0 & 0 \\
0 & 0 & 0.9997 & 0 & 0 & 0.0245 & 0 & 0.0082 & 0 \\
0.0050 & -0.5134 & 0 & 0.0574 & -0.6777 & -151.4 & 8.86 & 32.08 & 0 \\
0.0003 & -0.0438 & 0.2999 & 0 & 0.2803 & -1.7270 & -0.3438 & 0 & 0 \\
-0.0201 & -0.8670 & 0.7788 & 0 & -0.8383 & 2.3290 & -6.1570 & 0 & 0 \\
0 & 0 & -0.0018 & -0.0083 & 0 & 0.0728 & 1 & 0.0070 & 0 \\
0 & 0 & -0.0246 & -0.0006 & 0 & 1.002 & 0 & 0.0962 & 0
\end{array}\right],
\end{gathered}
$$




$$
\begin{gathered}
B_{1}=\left[\begin{array}{cc}
-0.0011 & -0.0098 \\
-0.8699 & -0.1217 \\
-1.0860 & 0 \\
0 & 0 \\
0 & 0.6157 \\
0 & -0.5951 \\
0 & 0.3826 \\
0 & 0 \\
0 & 0
\end{array}\right], B_{2}=\left[\begin{array}{ccccc}
0.0007 & -0.0086 \\
-0.8875 & -0.1349 \\
-1.0690 & -0.0015 \\
0 & 0 \\
0 & 0.6269 \\
0.0056 & -0.6114 \\
0.1264 & 0.4697 \\
0 & 0 \\
0 & 0
\end{array}\right] . \\
C=\left[\begin{array}{lllllllll}
0 & 0 & 0 & 1 & 0 & 0 & 0 & 0 & 0 \\
0 & 0 & 0 & 0 & 0 & 0 & 0 & 0 & 1
\end{array}\right] .
\end{gathered}
$$

Verification of design conditions. The invariant properties of interactor matrix and signs of leading principal minors have been shown by the generic linearized models. Here, the numerical values of the system parameters will be used to further verify the design conditions. It can be verified that all zeros of $G_{1}(s)=C\left(s I-A_{1}\right)^{-1} B_{1}$ and $G_{2}(s)=C\left(s I-A_{2}\right)^{-1} B_{2}$ have negative real parts, and $G_{1}(s)$ and $G_{2}(s)$ are strictly proper, and have full rank. It can also be verified that a common interactor matrix for both $G_{1}(s)$ and $G_{2}(s)$ is $\xi_{m}(s)=\operatorname{diag}\left\{(s+1)^{2},(s+1)^{2}\right\}$, such that the high frequency gain matrix for the nominal case is

$$
K_{p 1}=\lim _{s \rightarrow \infty} \xi_{m}(s) G_{1}(s)=\left[\begin{array}{cc}
-1.086 & 0 \\
0 & -0.596
\end{array}\right]
$$

and the high frequency gain matrix for the damage case is

$$
K_{p 2}=\lim _{s \rightarrow \infty} \xi_{m}(s) G_{2}(s)=\left[\begin{array}{cc}
-1.069 & 0.032 \\
-0.017 & -0.612
\end{array}\right] .
$$

The signs of first leading principal minor of $K_{p 1}$ and $K_{p 2}$ are

$$
\operatorname{sign}\left(\Delta_{11}\right)=\operatorname{sign}\left(\Delta_{21}\right)=-1,
$$

and the signs of second leading principal minor are

$$
\operatorname{sign}\left(\Delta_{21}\right)=\operatorname{sign}\left(\Delta_{22}\right)=1,
$$


which verifies there is no sign change of the principal minors.

Reference model. From the common interactor matrix $\xi_{m}(s)$ for both nominal and damage cases, the transfer matrix of the reference model (4.1.5) is chosen as $W_{m}(s)=\xi_{m}^{-1}(s)=\operatorname{diag}\left\{1 /(s+1)^{2}, 1 /(s+1)^{2}\right\}$.

Design parameters. Since the degree of $\xi_{m}(s)$ is 2 , the filter is chosen as $h(s)=$ $1 /(s+8)^{2}$. For the adaptive laws (4.3.19)-(4.3.21), the gain matrices are chosen as $\Gamma_{\theta 2}=10, \Gamma=\operatorname{diag}\{10,10\}$, and $D_{s}=\operatorname{diag}\{-30,-30\}$ because of the no sign change property of the principal minors.

Simulation results. To make a reasonable aircraft flying trajectory, a constant reference input is chosen as $r(t)=r_{1}(t)=[8 \pi / 180,15 \pi / 180]^{T}$. By applying the control law (4.3.1) with the adaptive laws (4.3.19)-(4.3.21), the output signal $\Delta y(t)$ (solid) tracks the reference signal $\Delta y_{m}(t)$ (dotted) after damage occurs at 200 second from Fig. 4.2.
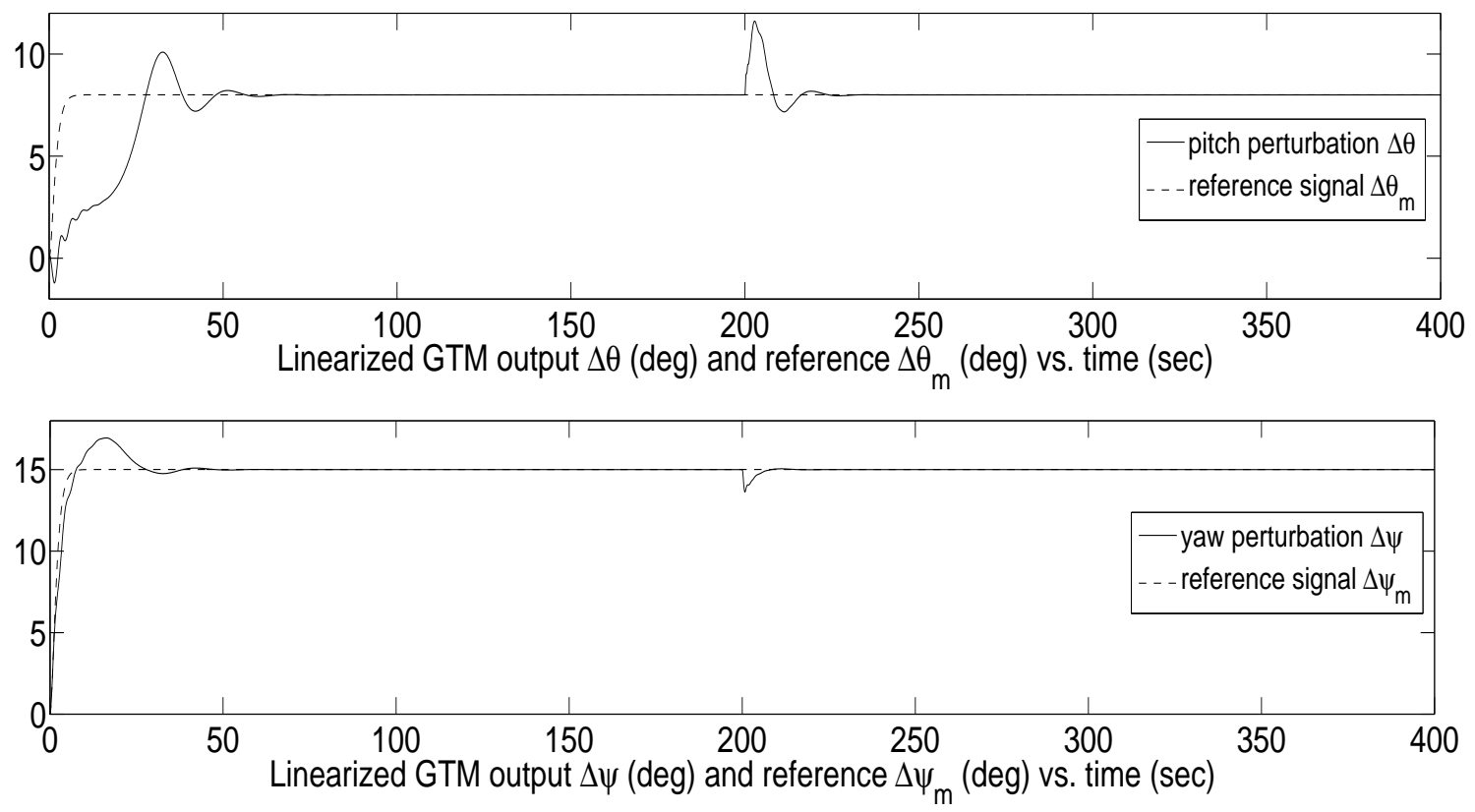

Figure 4.2: Linearized GTM output $\Delta y(t)$ (solid) vs. $\Delta y_{m}(t)($ dotted $)\left(r(t)=r_{1}(t)\right)$. 
Moreover, when the reference inputs are varying, the similar simulation results can be obtained as well. A varying reference input is chosen as $r(t)=r_{2}(t)=$ $[8 \pi / 180 \sin (0.015 t), 8 \pi / 180 \sin (0.015 t)]^{T}$. From Fig. 4.3, the output signals (solid) track the reference signals (dotted) after damage happens at 500 second.
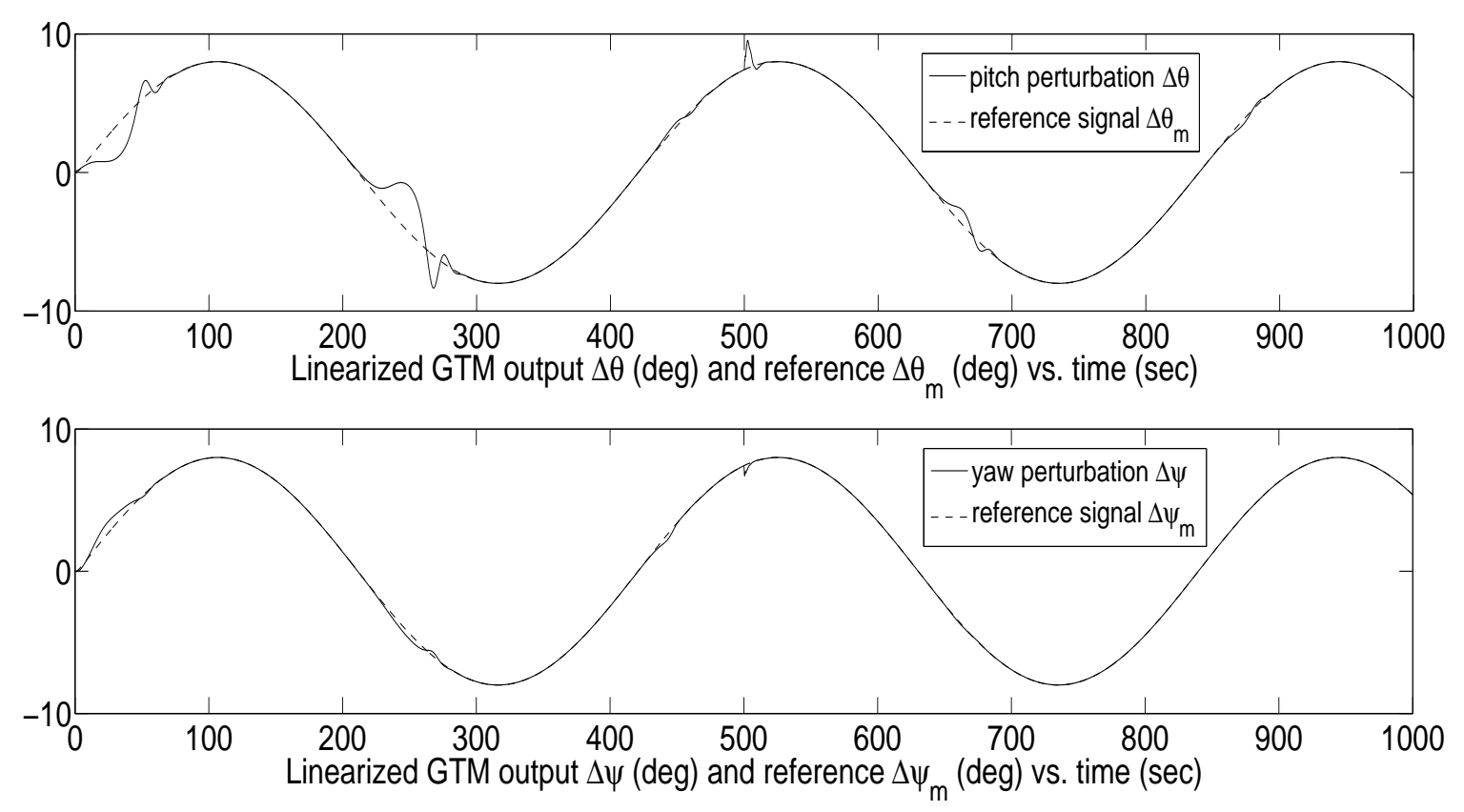

Figure 4.3: Linearized GTM output $\Delta y(t)$ (solid) vs. $\Delta y_{m}(t)\left(\right.$ dotted) $\left(r(t)=r_{2}(t)\right)$.

\subsubsection{Control of the Nonlinear GTM}

In the previous subsection, the linear control law $\Delta u(t)$ (4.3.1) is obtained. Then, $u(t)=\left[\Delta d_{e}(t)+d_{e 0}, \Delta d_{r}(t)+d_{r 0}, d_{a 0}, d_{t l 0}, d_{t r 0}\right]^{T}$ can be applied to the nonlinear GTM around the operating point $\left(x_{0}, u_{0}\right)$. In addition to show the aircraft output signal $y(t)=\Delta y(t)+C x_{0}=\left[\theta(t)+\theta_{0}, \psi(t)+\psi_{0}\right]^{T}$, the lateral-directional motion-roll angle $\phi(t)=\Delta \phi(t)+\phi_{0}$, control positions $d_{e}(t)=\Delta d_{e}(t)+d_{e 0}$ and $d_{r}(t)=\Delta d_{r}(t)+d_{r 0}$, and control gains $K_{1}(t), K_{2}(t)$, and $k_{3}(t)$, will be illustrated to verify that the aircraft can execute the maneuvers around the chosen operating condition $\left(x_{0}, u_{0}\right)$. Two operating 
conditions are considered: for case $\mathrm{I},\left(x_{0}, u_{0}\right)$ is obtained by trimming the nominal GTM to a wings-level flight condition with equivalent airspeed as 90 knots and roll angle as 0 radian; for case II, $\left(x_{0}, u_{0}\right)$ is obtained with equivalent airspeed as 100 knots and roll angle as 0 radian.

Case I. In the simulation, the wing-tip damage occurs at 200 second. From Fig. 4.4, it can be seen that the GTM output signals-pitch angle $\theta(t)$ and yaw angle $\psi(t)$ track the reference output signals $\theta_{m}(t)$ and $\psi_{m}(t)$, and the GTM state signalroll angle $\phi(t)$ is bounded before and after damage occurs. Moreover, the controller positions- $d_{e}(t)$ and $d_{r}(t)$ are within the GTM limits as shown in Fig. 4.5, while aileron and throttles are set as operating values. Fig. 4.6 gives time histories of some elements of $K_{1}(t), K_{2}(t)$, and $k_{3}(t)$.
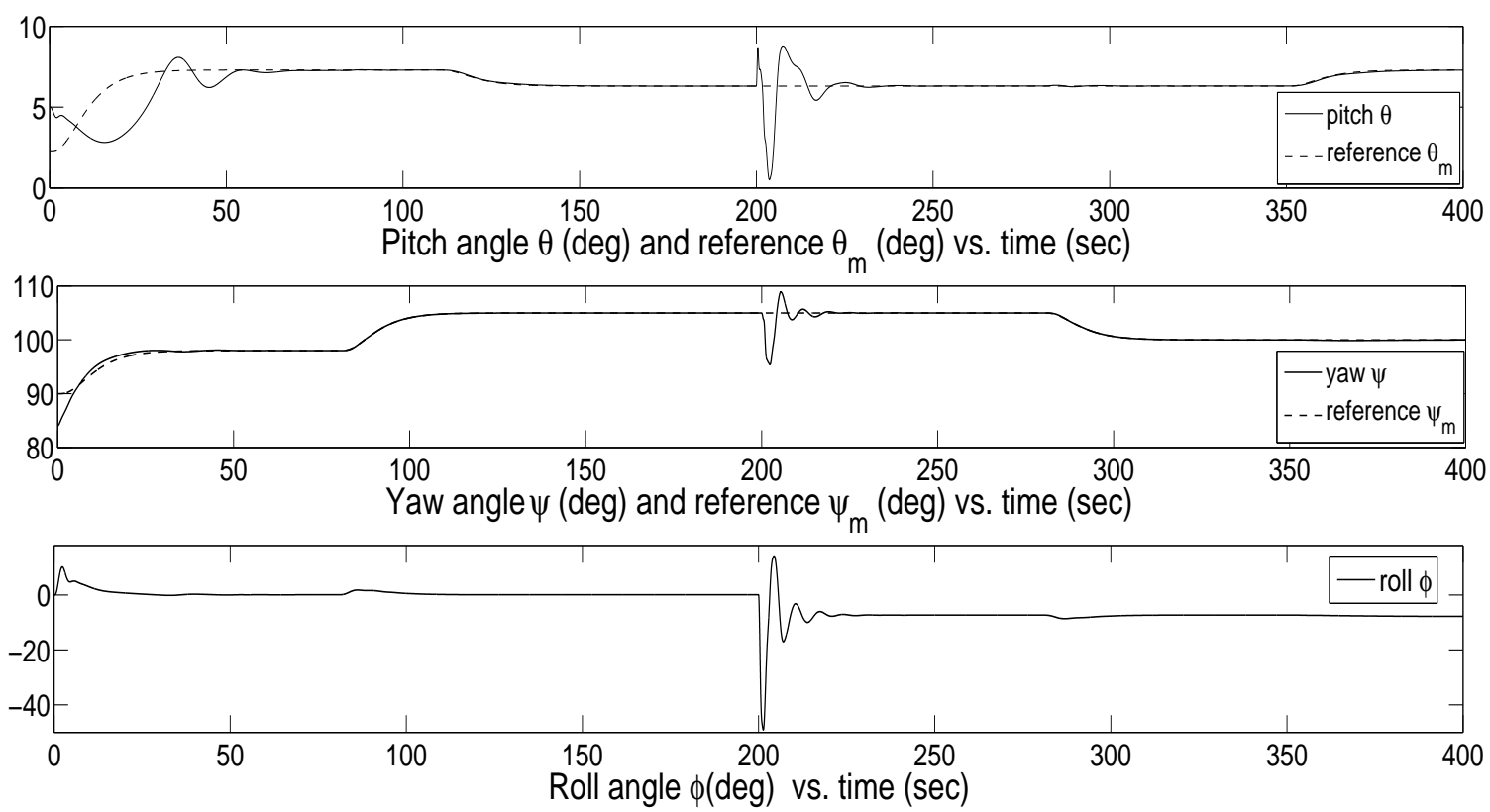

Figure 4.4: Responses of the GTM: pitch $\theta(t)$, yaw $\psi(t)$, and roll $\phi(t)$ (Case I).

Case II. To further demonstrate the effectiveness of the proposed linearizationbased design, it is applied to the GTM with damage around another operating point 

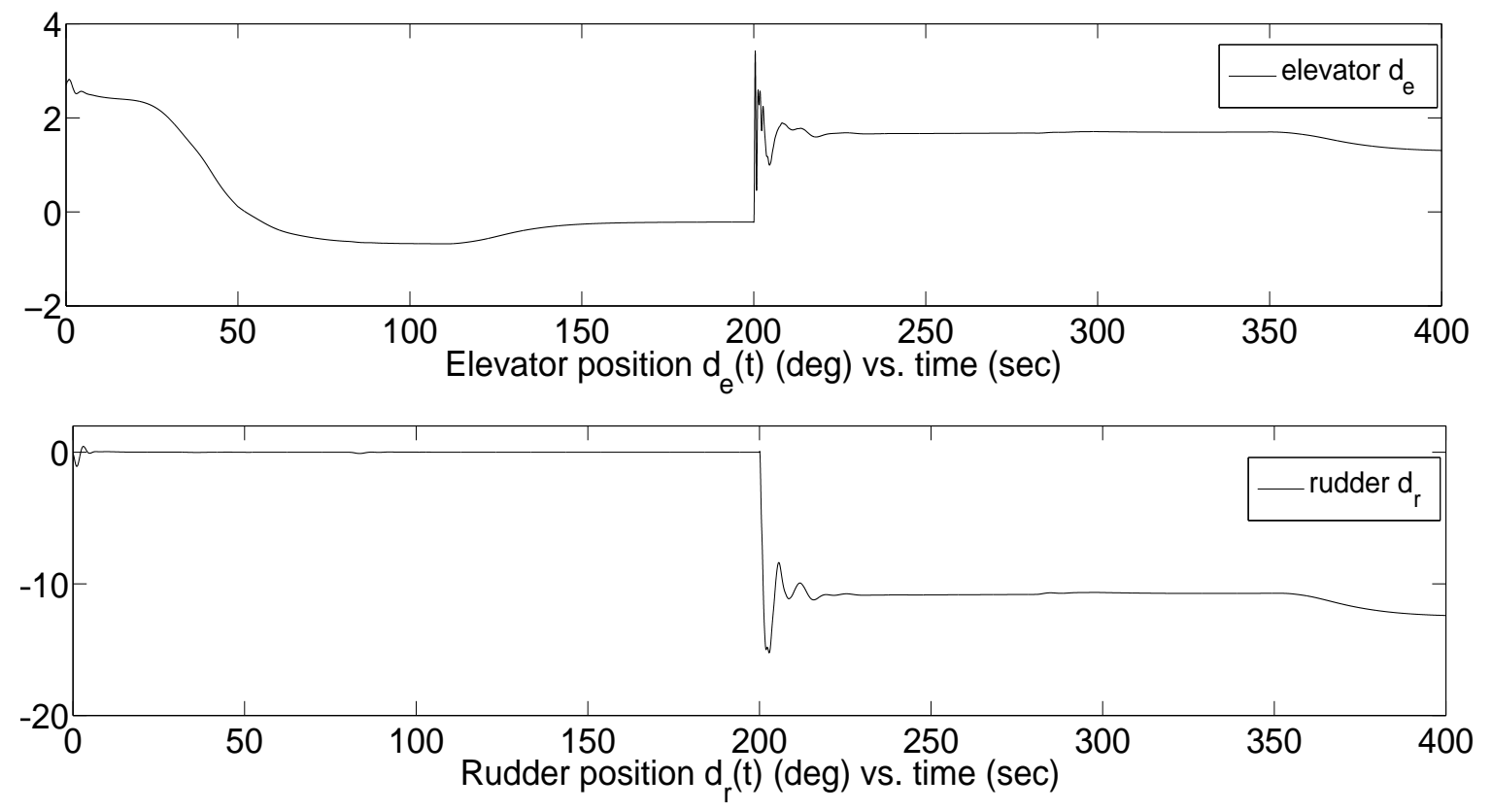

Figure 4.5: Control surface positions: elevator $d_{e}(t)$ and rudder $d_{r}(t)$ (Case I).
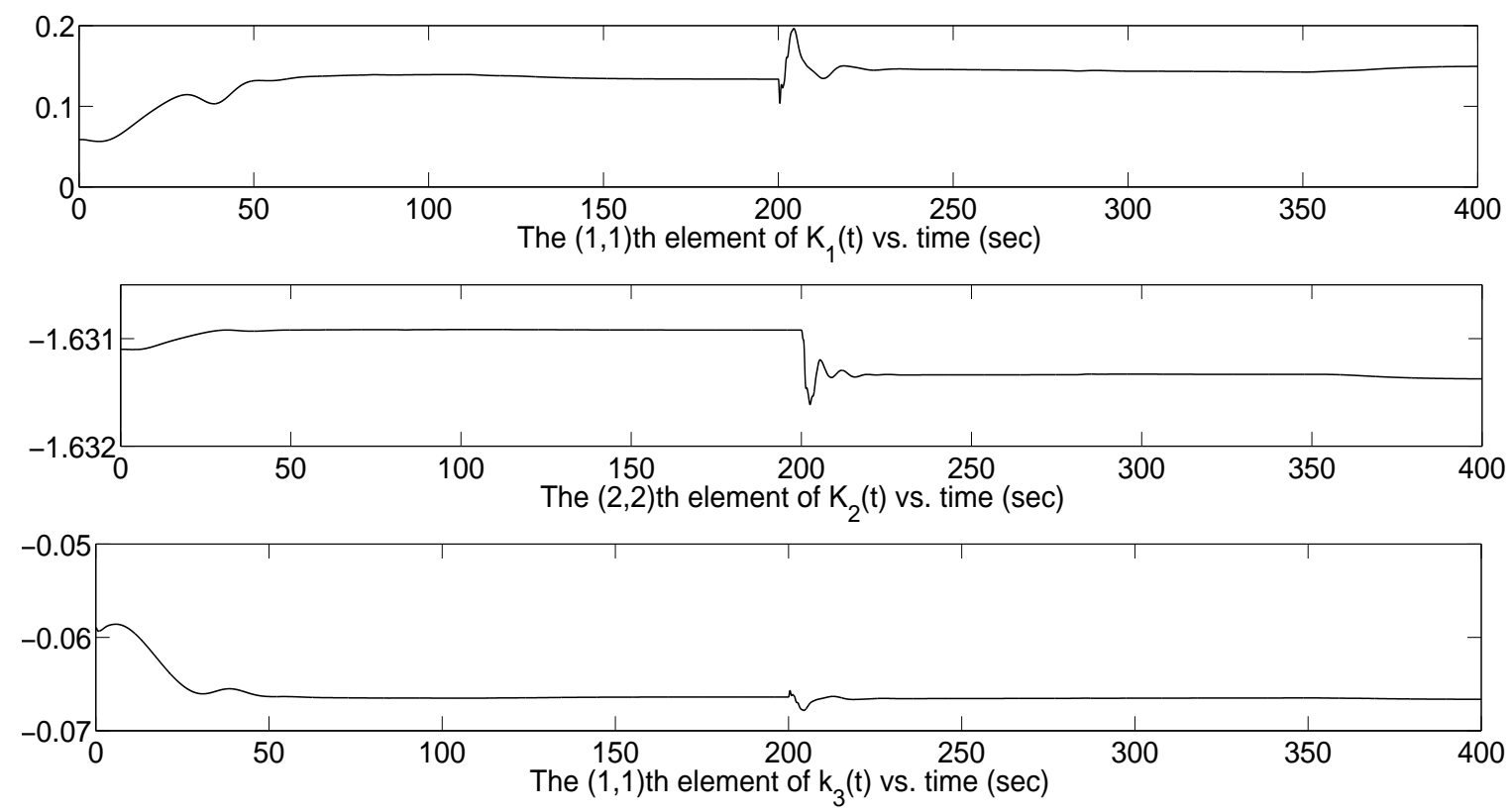

Figure 4.6: Elements of controller parameters $K_{1}(t), K_{2}(t)$, and $k_{3}(t)$ (Case I). 
$\left(x_{0}, u_{0}\right)$ obtained by trimming the nominal GTM to a wings-level flight condition with equivalent airspeed as 100 knots and roll angle as 0 radian. In the simulation, the wing-tip damage occurs at 200 second. Fig. 4.7 shows the GTM output signals $\theta(t)$ and $\psi(t)$, which follow the desired reference signals, and the GTM state signal $\phi(t)$, which is bounded within a reasonable boundary. The control surface positions $d_{e}(t)$ and $d_{r}(t)$ are shown in Fig. 4.8, which can be achieved by the GTM. In Fig. 4.9, some elements of the controller parameters $K_{1}(t), K_{2}(t)$, and $k_{3}(t)$ are illustrated.
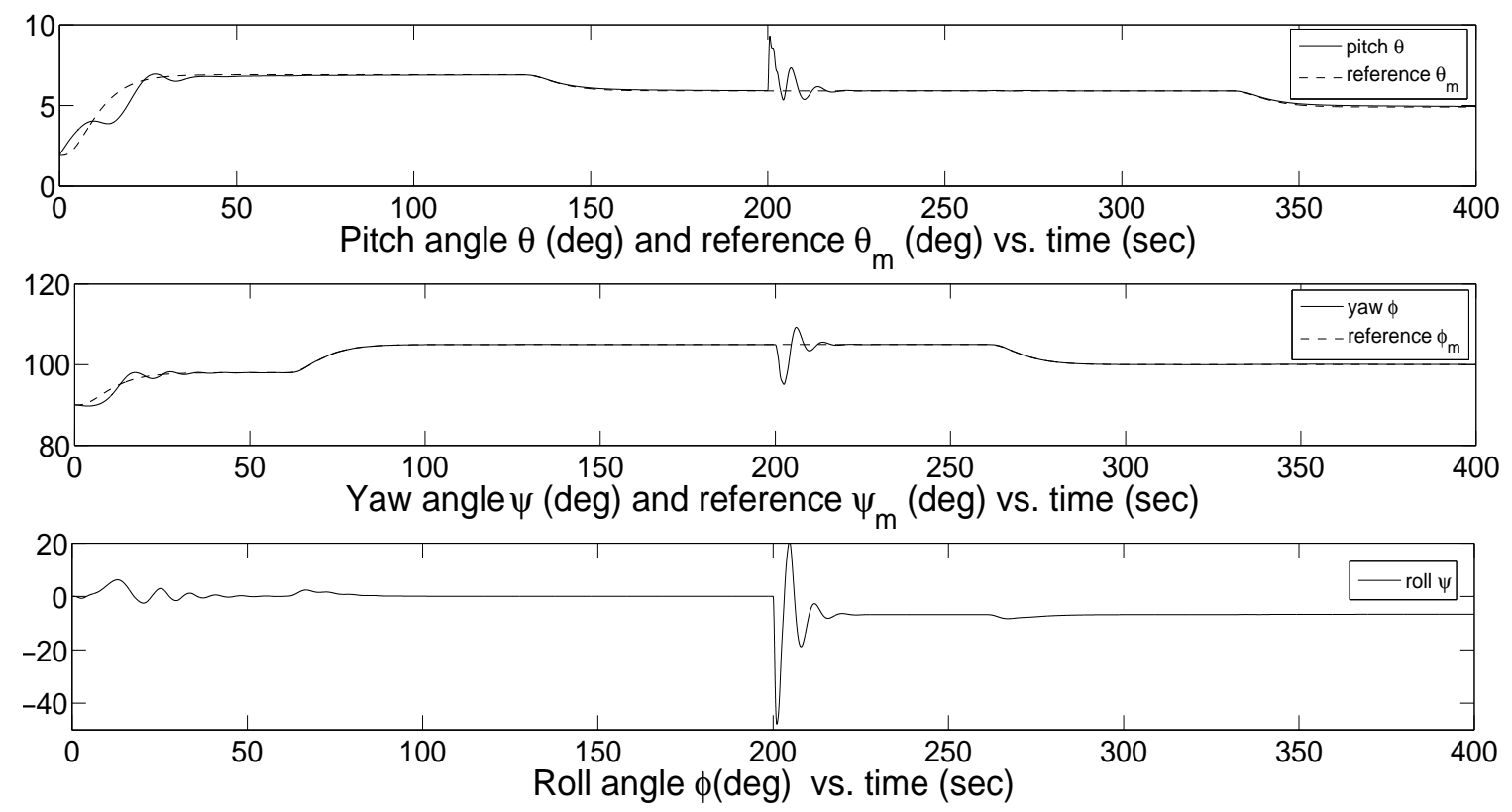

Figure 4.7: Responses of the GTM: pitch $\theta(t)$, yaw $\psi(t)$, and roll $\phi(t)$ (Case II).

Summary. From the simulation results in Case I and Case II, it can be concluded that the proposed linearization-based adaptive control design is applicable for the nonlinear aircraft system around a small neighborhood of the operating point $\left(x_{0}, u_{0}\right)$. Furthermore, the results in Case I and Case II indicate that one adaptive control design is effective around different operating conditions, so that the future research will address the expansion of operating domain for the proposed linearization-based 

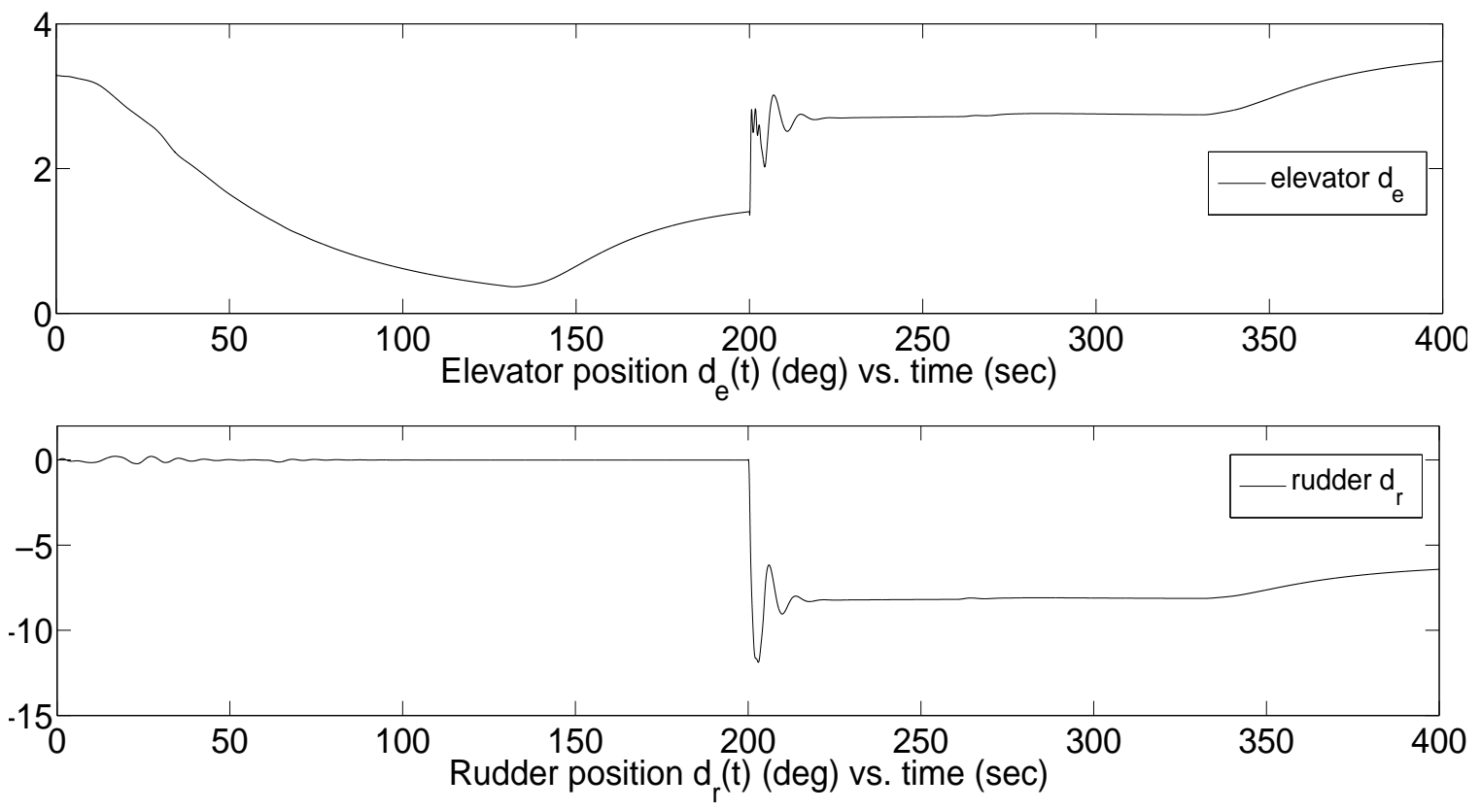

Figure 4.8: Control surface positions: elevator $d_{e}(t)$ and rudder $d_{r}(t)$ (Case II).
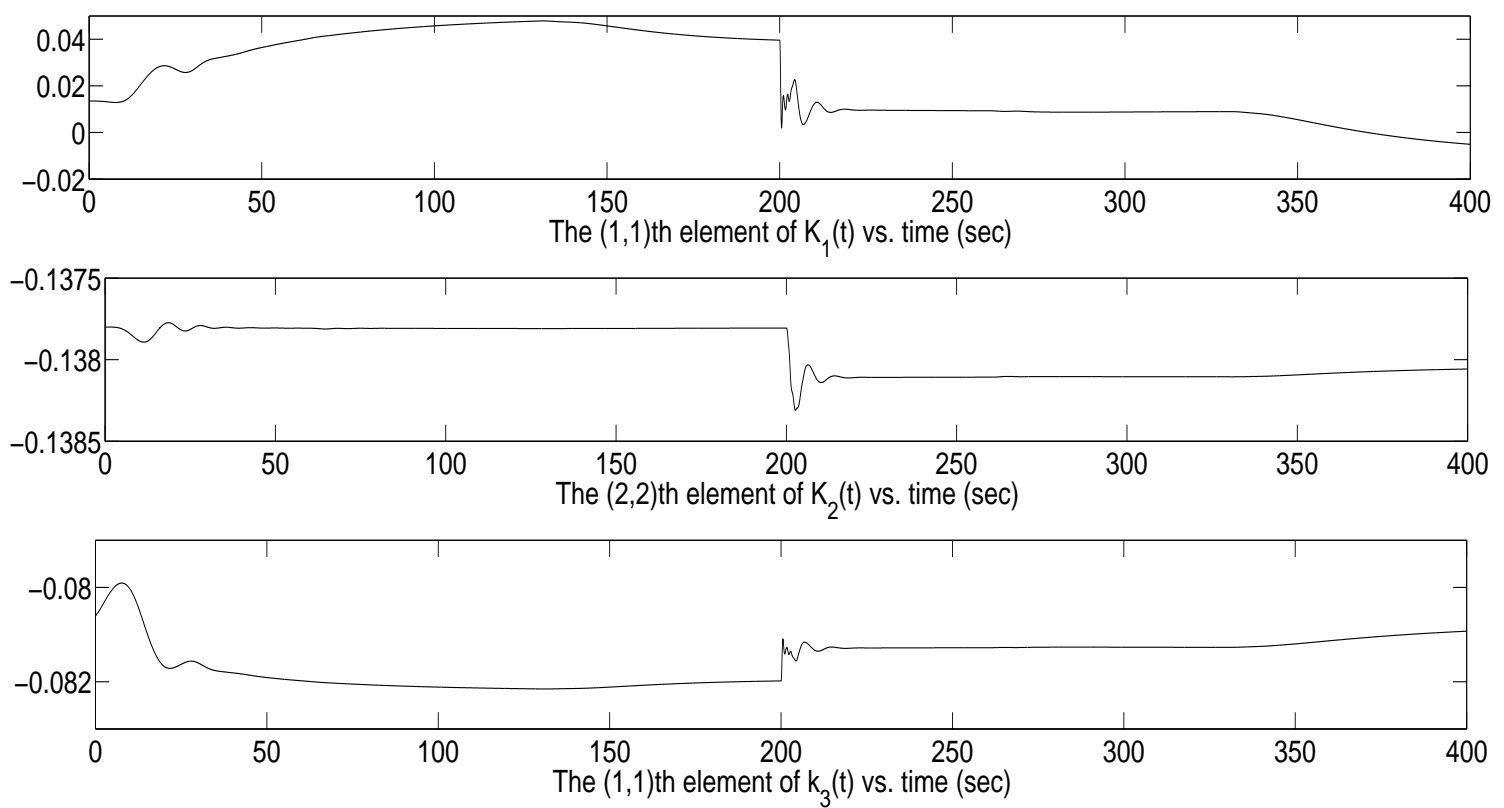

Figure 4.9: Elements of controller parameters $K_{1}(t), K_{2}(t)$, and $k_{3}(t)$ (Case II). 
control scheme.

\section{Summary}

In this chapter, a linearization-based multivariable adaptive control design for the nonlinear NASA Generic Transport Model (GTM) with damage has been developed. The nonlinear GTM with damage is linearized at a given operating point, and a sequential linear model with parameter uncertainties and a dynamics offset is obtained to represent the linearized aircraft system with damage. Based on the invariant properties of the system infinite zero structures and signs of high frequency gain matrices of the linearized aircraft system before and after damage, the multivariable model reference adaptive control (MRAC) scheme using state feedback has been developed. Due to the achievable plant-model matching conditions, the proposed MRAC design can reject the dynamics offset and make the signals of the close-loop system bounded and the output signals track some chosen reference signals before and after damage occurs. Both the stability analysis and the simulation results from the linearized and nonlinear GTMs have demonstrated certain desired system performance. 


\section{Chapter 5}

\section{Adaptive Simultaneous Actuator Failure and Structural Damage Compensation for MIMO Systems}

This chapter studies design and evaluation of a multivariable model reference adaptive control scheme for aircraft systems under simultaneous actuator failures and structural damage, where we

- obtain the invariance properties of the infinite zero structure for the linearized aircraft system under actuator failures and structural damage;

- derive a less restrictive plant-model matching condition that can be satisfied under actuator failures and structural damage;

- develop a multivariable MRAC scheme using output feedback to guarantee the closed-loop system stability and asymptotic output tracking before and after failures and damage occur;

- verify the effectiveness of this adaptive linearization-based control design on the nonlinear aircraft system by extensive simulation studies on the high-fidelity nonlinear GTM Simulink model. 


\subsection{Problem Statement}

Consider the nonlinear aircraft flight system denoted as

$$
\dot{x}(t)=f(x(t), u(t)), \quad y(t)=C x(t),
$$

which is constructed by force equations, moment equations and kinematic equations as shown in Section 2.1 from (2.1.1)-(2.1.9). When damage occurs, the structure and parameters of the system dynamics function $f(x, u)$ are under unknown changes. When actuators fail, the control input signal $u(t)$ undergoes undesired performance, which can be described as

$$
u(t)=\left(I_{m}-\sigma\right) v(t)+\sigma \bar{u}(t)
$$

where $v(t)$ is a commanded control input vector signal, $\bar{u}(t)$ is an unknown input vector signal due to actuator failures, and the elements of the failure index $\sigma=$ $\operatorname{diag}\left\{\sigma_{1}, \sigma_{2} \ldots, \sigma_{m}\right\}$ are $\sigma_{i}=1$ if the $i$ th actuator fails or $\sigma_{i}=0$ otherwise, $i=$ $1,2, \ldots, m$ with $m$ being the dimension of the control signal $u(t)$. In this chapter, we only consider the lock-in-place failures, that is $\bar{u}(t)=\bar{u}$ with $\bar{u}$ being a constant unknown failure vector.

Linearization-based design. To compensate the damage and actuator failures, a linearization-based control design will be applied to the nonlinear aircraft flight system (5.1.1). Since there are uncertainties for the aircraft system with damage, the equilibrium point is not available for the damaged system. Therefore, a chosen operating point $\left(x_{0}, u_{0}\right)$, which may not be an equilibrium point, is used to obtain the linearized system for the system (5.1.1) with damage and actuator failures (5.1.2):

$$
\begin{aligned}
\Delta \dot{x}(t) & =A \Delta x(t)+B \Delta u(t)+f_{0}, \Delta y(t)=C \Delta x(t), \\
\Delta u(t) & =\left(I_{m}-\sigma\right) \Delta v(t)+\sigma \Delta \bar{u},
\end{aligned}
$$


where $\Delta x=x-x_{0}, \Delta y=y-C x_{0}, \Delta u=u-u_{0}, \Delta v=v-u_{0}$ and $\Delta \bar{u}=\bar{u}-u_{0}$ are perturbation signals, and $A=\partial f /\left.\partial x\right|_{\left(x_{0}, u_{0}\right)}, B=\partial f /\left.\partial u\right|_{\left(x_{0}, u_{0}\right)}, f_{0}=f\left(x_{0}, u_{0}\right)$ are unknown piecewise constant parameters and dynamics offset due to different damage conditions, and the failure index $\sigma$ is also an uncertain piecewise constant due to different failure patterns. The detailed structures of parameters $A, B, \sigma$, and some important properties of the linearized aircraft system will be studied later. After deriving the adaptive control law $\Delta v(t)$ for the linearized aircraft system (5.1.3), the control law $v(t)=\Delta v(t)+u_{0}$ can be applied to the original nonlinear system (5.1.1) with damage and actuator failures in the small neighborhood of $\left(x_{0}, u_{0}\right)$.

Sequential linear model. Since the design is based on the linearized aircraft system (5.1.3), in this chapter, a sequential linear model with the structural damage and actuator failures is considered:

$$
\begin{aligned}
\dot{x}(t) & =A x(t)+B u(t)+f_{0}, y(t)=C x(t), \\
u(t) & =\left(I_{m}-\sigma\right) v(t)+\sigma \bar{u},
\end{aligned}
$$

where $x(t) \in R^{n}, y(t) \in R^{M}$ and $u(t) \in R^{m}$ are state, output and input vector signals with $m>M$ (actuation redundancy), $v(t) \in R^{m}$ is a commanded control input vector signal, and $\bar{u}$ is an unknown constant failure vector. Assume that, within each time interval $\left[t_{k-1}, t_{k}\right)$, with $t_{0}=0, t_{N}=\infty$, and $t_{k}, k=2, \ldots, N-1$, being unknown, the damage and failure pattern is fixed, such that $\left(A, B, C, f_{0}\right)=\left(A_{k}, B_{k}, C_{k}, f_{0 k}\right)$ with $A_{k}, B_{k}, C_{k}$, and $f_{0 k}$ being unknown constants and $\sigma=\operatorname{diag}\left\{\sigma_{k 1}, \sigma_{k 2}, \ldots, \sigma_{k m}\right\}$ with $\sigma_{k j}=1$ if the $j$ th actuator fails or $\sigma_{k j}=0$ otherwise.

Actuation redundancy and grouping. The $m$ actuators are divided into $M$ groups, within which the actuators have the same physical characteristics. We apply a proportional actuation scheme for the commanded input signals $v_{i j}(t), i=1, \ldots, M$ and $j=1, \ldots, n_{i}$ within each group: $v_{i j}(t)=\alpha_{i j} v_{i 0}(t)$, where $v_{i 0}(t) \in R$ is the 
designed input signal for the corresponding group. Then, the commanded control input vector signal $v(t)$ can be expressed as

$$
v(t)=H v_{0}(t)
$$

where the designed input vector signal $v_{0}(t)$ is $v_{0}(t)=\left[v_{10}, v_{20}, \ldots, v_{M 0}\right]^{T} \in R^{M}$ and $H=\operatorname{diag}\left\{H_{1}, H_{2}, \ldots, H_{M}\right\}$ with $H_{i}=\left[\alpha_{i 1}, \alpha_{i 2}, \ldots, \alpha_{i n_{i}}\right]^{T}$ for $i=1, \ldots, M$. Applying (5.1.5) to the system (5.1.4), we have

$$
y(t)=G_{a}(s)\left[v_{0}\right](t)+\bar{y}(t)
$$

where $G_{a}(s)=C(s I-A)^{-1} B_{a}$ with $B_{a}=B\left(I_{m}-\sigma\right) H$ is the unknown transfer matrix (due to damage and failures) from the healthy actuators to the output, and $\bar{y}(t)=C(s I-A)^{-1} B \sigma[\bar{u}](t)+C(s I-A)^{-1} f_{0}\left[h_{f}\right](t)$ with $h_{f}(t)$ being a unit step signal is the uncertain output due to the actuator failures and the dynamics offset. It is worth noting that, to compensate the damage and actuator failures, we assume that there is at least one working actuator in each group and columns of $B$ matrix corresponding to the working actuators in each group are not 0 (since a column of $B$ with all entries being 0 means that the corresponding actuator is lost caused by airframe damage).

Control objective. The control objective is to design an output feedback control law $v_{0}(t)=\left[v_{10}, v_{20}, \ldots, v_{M 0}\right]^{T}$ for the system (5.1.6) to compensate the system uncertainties caused by damage and actuator failures and make all the closed-loop system signals bounded and the output signal $y(t)$ track a reference signal:

$$
y_{m}(t)=W_{m}(s)[r](t)
$$

where $W_{m}(s) \in R^{M \times M}$ is a stable transfer matrix and $r(t) \in R^{M}$ is a bounded reference input. For our multivariable MRAC design, we choose the reference system

$$
W_{m}(s)=\xi_{m}^{-1}(s)
$$


Based on the knowledge of the infinite zero structure, i.e., the interactor matrix and the high frequency gain matrix, the plant-model matching condition (2.4.3) can be established to achieve output tracking of $y_{m}(t)$ from (5.1.7).

Design assumptions. To proceed the design, for the system (5.1.6) with all possible damage and failure patterns $\left(A, B, C, f_{0}, \sigma\right)=\left(A_{k}, B_{k}, C_{k}, f_{0 k}, \sigma_{k}\right), k=1, \ldots, N$, the following assumptions are required: (A1) $G_{a}(s)$ has full rank and all zeros of $G_{a}(s)$ have negative real parts; (A2) an upper bound $\bar{\nu}_{0}$ of the observability indices of all $G_{a}(s)$ is known; (A3) for $G_{a}(s)=P_{l}^{-1}(s) Z_{l a}(s), C(s I-A)^{-1} B \sigma=P_{l}^{-1}(s) Z_{l b}(s)$, and $C(s I-A)^{-1} f_{0}=P_{l}^{-1}(s) Z_{f}(s)$, the transfer matrices $Z_{l a}^{-1}(s) Z_{l b}(s)$ and $Z_{l a}^{-1}(s) Z_{f}(s)$ are proper; (A4) there is a known modified left interactor matrix $\xi_{m}(s)$ for all $G_{a}(s)$, which is invariant for all the damage and failure patterns; (A5) all leading principal minors $\Delta_{i}, i=1,2 \ldots, M$, of the high frequency gain matrix $K_{p a}=\lim _{t \rightarrow \infty} \xi_{m}(s) G_{a}(s)$ are nonzero and the signs are known and invariant for all the damage and failure patterns.

Assumptions (A1), (A2), and (A3) ensure the existence of plant-model matching between the system and the reference model. The assumption (A4) ensures that there is a uniform reference system $W_{m}(s)=\xi_{m}^{-1}(s)$ for all uncertain adverse conditions, and (A5) ensures that the uncertainties of $K_{p a}$ can be handled for all different damage and failure conditions when developing the adaptive control scheme.

Before developing adaptive control to compensate the failures and damage, in the next section, we will investigate invariance of the interactor matrix $\xi_{m}(s)$ and signs of the leading principal minors of $K_{p a}$ of linearized aircraft models to show that the aircraft system can satisfy the design conditions. 


\subsection{System Invariance of An Aircraft Model}

The operating point for linearization of the aircraft system (5.1.1) is chosen as a wings-level flight condition $\left(x_{0}, u_{0}\right)$ with

$$
x_{0}=\left[u_{b 0}, w_{b 0}, 0, \theta_{0}, 0,0,0,0, \psi_{0}\right]^{T},
$$

which may not be an equilibrium due to damage. For the aircraft system (5.1.1), we choose the output as

$$
y(t)=C x(t)=[\theta(t), \psi(t)]^{T}
$$

and the control input as

$$
u(t)=\left[d_{e}^{T}(t), d_{r}^{T}(t)\right]^{T},
$$

where $d_{e}(t)=\left[d_{e_{l_{1}}}, d_{e_{l_{2}}}, d_{e_{r_{1}}}, d_{e_{r_{2}}}\right]^{T}$ is the elevator group with $d_{e_{l_{1}}}, d_{e_{l_{2}}}, d_{e_{r_{1}}}$ and $d_{e_{r_{2}}}$ being deflections of elevator left outboard, left inboard, right outboard and right inboard segments, and $d_{r}(t)=\left[d_{r_{u}}, d_{r_{l}}\right]^{T}$ is the rudder group with $d_{r_{u}}$ and $d_{r_{l}}$ being deflections of rudder upper and lower segments. Then, for the linearized aircraft system (5.1.3) applying the proportional actuation scheme (5.1.5): $\Delta v(t)=H \Delta v_{0}(t)$, where $H=\operatorname{diag}\left\{H_{1}, H_{2}\right\}$ with $H_{1}=[1,1,1,1]^{T}$ and $H_{2}=[1,1]^{T}$ to the elevator and rudder groups, we have

$$
\Delta y(t)=G_{a}(s)\left[\Delta v_{0}\right](t)+\Delta \bar{y}(t)
$$

where $G_{a}(s)=C(s I-A)^{-1} B_{a}$ with $A \in R^{9 \times 9}, B_{a}=\left[B_{a 1}, B_{a 2}\right]=B(I-\sigma) H \in R^{9 \times 2}$, and

$$
C=\left[\begin{array}{lllllllll}
0 & 0 & 0 & 1 & 0 & 0 & 0 & 0 & 0 \\
0 & 0 & 0 & 0 & 0 & 0 & 0 & 0 & 1
\end{array}\right]
$$

In the following, we will investigate the invariance of infinite structure of $G_{a}(s)$ under actuator failure and damage conditions based on generic structures of $A$ and $B_{a}$. 


\subsubsection{Generic Structures of the System Parameters}

From the generic nonlinear aircraft model under damage as in (2.1.1)-(2.1.9) and the possible actuator failure pattern $\sigma$, the generic structures of

$$
A=\partial f /\left.\partial x\right|_{\left(x_{0}, u_{0}\right)}, B_{a}=B(I-\sigma) H
$$

with $B=\partial f /\left.\partial u\right|_{\left(x_{0}, u_{0}\right)}$ of the linearized system (5.2.4) can be derived for the following four damage and failure cases.

$A$ and $B_{a}$ for the healthy case. When there is no damage, according to [57], generic structures of $A$ and $B$ of the linearized aircraft model (5.1.3) are

$$
A=\left[\begin{array}{cc}
A_{1}^{(4 \times 4)} & 0^{(4 \times 5)} \\
0^{(5 \times 4)} & A_{4}^{(5 \times 5)}
\end{array}\right], \quad B=\left[\begin{array}{cc}
B_{1}^{(3 \times 4)} & 0^{(3 \times 2)} \\
0^{(1 \times 4)} & 0^{(1 \times 2)} \\
0^{(3 \times 4)} & B_{4}^{(3 \times 2)} \\
0^{(2 \times 4)} & 0^{(2 \times 2)}
\end{array}\right]
$$

with $A_{1}$ and $A_{4}$ possessing the following structures:

$$
A_{1}=\left[\begin{array}{cccc}
a_{11} & a_{12} & a_{13} & a_{14} \\
a_{21} & a_{22} & a_{23} & a_{24} \\
a_{31} & a_{32} & a_{33} & a_{34} \\
0 & 0 & 1 & 0
\end{array}\right], A_{4}=\left[\begin{array}{ccccc}
a_{55} & a_{56} & a_{57} & a_{58} & 0 \\
a_{65} & a_{66} & a_{67} & a_{68} & 0 \\
a_{75} & a_{76} & a_{77} & a_{78} & 0 \\
0 & \tan \theta_{0} & 1 & 0 & 0 \\
0 & 1 / \cos \theta_{0} & 0 & 0 & 0
\end{array}\right] .
$$

Since no failures happen $(\sigma=0)$, we have $B_{a}=B H$.

$A$ and $B_{a}$ for the failure but no damage case. When there is no damage, the matrices $A$ and $B$ are given as (5.2.7). We assume that there are $q_{e}$ healthy elevators with $0<q_{e} \leq 4$ and $q_{r}$ healthy rudders with $0<q_{r} \leq 2$, that is

$$
B_{a}=B(I-\sigma) H, \sigma=\operatorname{diag}\left\{\sigma_{e_{1}}, \sigma_{e_{2}}, \sigma_{e_{3}}, \sigma_{e_{4}}, \sigma_{r_{1}}, \sigma_{r_{2}}\right\}
$$

where $\sigma_{e_{i}}=0$ with $i \in\left\{j_{1}, \ldots, j_{q_{e}}\right\} \subseteq\{1,2,3,4\}$ and $\sigma_{e_{i}}=1$ otherwise, and $\sigma_{r_{i}}=0$ with $i \in\left\{l_{1}, l_{q_{r}}\right\} \subseteq\{1,2\}$ and $\sigma_{r_{i}}=1$ otherwise. 
$A$ and $B_{a}$ for the damage but no failure case. When damage occurs, the longitudinal and lateral-directional dynamics are coupled. $A$ and $B$ become [57]

$$
A=\left[\begin{array}{ll}
A_{d 1}^{(4 \times 4)} & A_{d 2}^{(4 \times 5)} \\
A_{d 3}^{(5 \times 4)} & A_{d 4}^{(5 \times 5)}
\end{array}\right], \quad B=\left[\begin{array}{cc}
B_{d 1}^{(3 \times 4)} & B_{d 2}^{(3 \times 2)} \\
0^{(1 \times 4)} & 0^{(1 \times 2)} \\
B_{d 3}^{(3 \times 4)} & B_{d 4}^{(3 \times 2)} \\
0^{(2 \times 4)} & 0^{(2 \times 2)}
\end{array}\right],
$$

with elements and structures undergoing uncertain changes:

$$
\begin{aligned}
A_{d_{1}}= & {\left[\begin{array}{cccc}
a_{d_{11}} & a_{d_{12}} & a_{d_{13}} & a_{d_{14}} \\
a_{d_{21}} & a_{d_{22}} & a_{d_{23}} & a_{d_{24}} \\
a_{d_{31}} & a_{d_{32}} & a_{d_{33}} & a_{d_{34}} \\
0 & 0 & 1 & 0
\end{array}\right] A_{d_{2}}=\left[\begin{array}{ccccc}
a_{d_{15}} & a_{d_{16}} & a_{d_{17}} & a_{d_{18}} & 0 \\
a_{d_{25}} & a_{d_{26}} & a_{d_{27}} & a_{d_{28}} & 0 \\
a_{d_{35}} & a_{d_{36}} & a_{d_{37}} & a_{d_{38}} & 0 \\
0 & 0 & 0 & 0 & 0
\end{array}\right] } \\
A_{d_{3}}= & {\left[\begin{array}{cccc}
a_{d_{51}} & a_{d_{52}} & a_{d_{53}} & a_{d_{54}} \\
a_{d_{61}} & a_{d_{62}} & a_{d_{63}} & a_{d_{64}} \\
a_{d_{71}} & a_{d_{72}} & a_{d_{73}} & a_{d_{74}} \\
0 & 0 & 0 & 0 \\
0 & 0 & 0 & 0
\end{array}\right] A_{d_{4}}=\left[\begin{array}{ccccc}
a_{d_{55}} & a_{d_{56}} & a_{d_{57}} & a_{d_{58}} & 0 \\
a_{d_{65}} & a_{d_{66}} & a_{d_{67}} & a_{d_{68}} & 0 \\
a_{d_{75}} & a_{d_{76}} & a_{d_{77}} & a_{d_{78}} & 0 \\
0 & a_{d_{86}} & 1 & 0 & 0 \\
0 & \frac{1}{\cos \theta_{0}} & 0 & 0 & 0
\end{array}\right] . }
\end{aligned}
$$

In this case, no actuator lock-in-place failure happens, that is control input $u(t)=$ $v(t)$. However, some actuators may be partially or totally lost due to the structural damage. Such a loss can be represented by variations of entries of $B$ matrix from the nominal values to the damaged values. Particularly, if an actuator is totally lost, the corresponding column of $B$ matrix becomes 0 , that is such an actuator has no control effect on the aircraft system at all. Hence, the loss of effectiveness of actuators can be treated as a damage case. To compensate the damage, assume that not all the actuators in each group are totally lost to ensure that the column vectors of $B_{a}$ matrix are not 0 . Since no failure occurs, the index $\sigma=0$, it follows that $B_{a}=B H$.

$A$ and $B_{a}$ for the both damage and failure case. In this case, the remaining actuators after damage still suffer from the lock-in-place failures. Since damage occurs, $A$ and $B$ become the damaged values (5.2.9). To compensate the damage and failures, we assume that there are $q_{e}$ working elevators with $0<q_{e} \leq 4$ and $q_{r}$ working rudders with $0<q_{r} \leq 2$. The working actuator in this case means that it is 
not totally lost due to damage (so that the corresponding column of $B$ matrix is not 0) and does not suffer from the lock-in-place failure. With such an actuator failure and damage pattern, parameter $B_{a}$ is given as

$$
B_{a}=B(I-\sigma) H, \sigma=\operatorname{diag}\left\{\sigma_{e_{1}}, \sigma_{e_{2}}, \sigma_{e_{3}}, \sigma_{e_{4}}, \sigma_{r_{1}}, \sigma_{r_{2}}\right\}
$$

where $\sigma_{e_{i}}=0$ with $i \in\left\{j_{1}, \ldots, j_{q_{e}}\right\} \subseteq\{1,2,3,4\}$ and $\sigma_{e_{i}}=1$ otherwise, and $\sigma_{r_{i}}=0$ with $i \in\left\{l_{1}, l_{q_{r}}\right\} \subseteq\{1,2\}$ and $\sigma_{r_{i}}=1$ otherwise.

Then, we can investigate the two invariance properties for $G_{a}(s)$ based on the structures of $A$ and $B_{a}$.

\subsubsection{Invariance of Infinite Zero Structure}

From Lemma 2.3.1, to determine the infinite zero structure of $G_{a}(s)=C(s I-A)^{-1} B_{a}$, we need to study relative degrees of entries of $G_{a}(s)$, which is calculated as

$$
G_{a}(s)=\frac{1}{\alpha(s)}\left(E_{n-1} s^{n-1}+E_{n-2} s^{n-2}+\cdots+E_{1} s+E_{0}\right)
$$

where $\alpha(s)=\operatorname{det}(s I-A) \triangleq s^{n}+\alpha_{n-1} s^{n-1}+\cdots+\alpha_{1} s+\alpha_{0}, E_{n-1}=C B_{a}, E_{n-2}=$ $C A B_{a}+\alpha_{n-1} C B_{a}, \ldots, E_{0}=C A^{n-1} B_{a}+\alpha_{n-1} C A^{n-2} B_{a}+\cdots+\alpha_{1} C B_{a}$.

Infinite zero structure of the nominal case. The parameters $A$ and $B$ are given as (5.2.7) and $B_{a}=B H$. With the matrix $C$ given as (5.2.5), we have coefficients for $G_{a}(s)$ as

$$
E_{n-1}=0, E_{n-2}=\operatorname{diag}\left\{\sum_{i=1}^{4} b_{3 e i}, \frac{1}{\cos \theta_{0}}\left(b_{6 r 1}+b_{6 r 2}\right)\right\},
$$

with $b_{3 e i}, i=1, \ldots, 4$, and $b_{6 r i}, i=1,2$, being elements of the 3rd and 6th rows of the matrix $B$ corresponding to the elevators and rudders. Therefore, we can choose an interactor matrix for $G_{a}(s)$ as $\xi_{m}(s)=\operatorname{diag}\left\{(s+1)^{2},(s+1)^{2}\right\}$, so that the high frequency gain matrix can be obtained as $K_{p a}=\lim _{s \rightarrow \infty} \xi_{m}(s) G_{a}(s)=E_{n-2}$. Since 
the parameters $b_{3 e 1}, \ldots, b_{3 e 4}, b_{6 r 1}$, and $b_{6 r 2}$ are the control gains from elevators to pitch acceleration and rudders to yaw acceleration, the signs of these parameters can be obtained: $b_{3 e 1}, \ldots, b_{3 e 4}<0, b_{6 r 1}<0$, and $b_{b r 2}<0$. The operating point is chosen as $\theta_{0} \in(-\pi / 2, \pi / 2)$. That is the signs of the leading principal minors are $\operatorname{sign}\left(\Delta_{1}\right)=-1, \operatorname{sign}\left(\Delta_{2}\right)=1$.

Case I: failures occur before damage happens. When failures occur, the matrix $A$ is still the nominal value given in (5.2.7), but the matrix $B_{a}$ changes to (5.2.8) with certain failure patterns. $E_{n-1}$ and $E_{n-2}$ for $G_{a}(s)$ are calculated as

$$
E_{n-1}=0, E_{n-2}=\operatorname{diag}\left\{\sum_{i=j_{1}, \ldots, j_{q_{e}}} b_{3 e i}, \frac{1}{\cos \theta_{0}} \sum_{i=l_{1}, l_{q_{r}}} b_{6 r i}\right\} .
$$

Therefore, we can choose $\xi_{m}(s)$ as $\xi_{m}(s)=\operatorname{diag}\left\{(s+1)^{2},(s+1)^{2}\right\}$, so that $K_{p a}=$ $\lim _{s \rightarrow \infty} \xi_{m}(s) G_{a}(s)=C A B_{a}$. Since there is no damage, the parameters $b_{3 e i}<0, i \in$ $\left\{j_{1}, \ldots, j_{q_{e}}\right\}$ and $b_{6 r i}<0, i \in\left\{l_{1}, l_{q_{r}}\right\}$, it follows that $\operatorname{sign}\left(\Delta_{1}\right)=-1, \operatorname{sign}\left(\Delta_{2}\right)=1$.

After damage happens, the aircraft suffers from the simultaneous failures and damage condition. The matrices $A$ and $B_{a}$ become the damaged values (5.2.9) and (5.2.10), while the failure patterns $\left\{j_{1}, \ldots, j_{q_{e}}\right\}$ and $\left\{l_{1}, l_{q_{r}}\right\}$ for $B_{a}$ do not change. The coefficients are calculated as $E_{n-1}=0$ and

$$
E_{n-2}=\left[\begin{array}{cc}
\sum_{i=j_{1}, \ldots, j_{q_{e}}} b_{d 3 e i} & \sum_{i=l_{1}, l_{q_{r}}} b_{d 3 r i} \\
\frac{1}{\cos \theta_{0}} \sum_{i=j_{1}, \ldots, j_{q_{e}}} b_{d 6 e i} & \frac{1}{\cos \theta_{0}} \sum_{i=l_{1}, l_{q_{r}}} b_{d 6 r i}
\end{array}\right] .
$$

Therefore, the interactor matrix can still be $\xi_{m}(s)=\operatorname{diag}\left\{(s+1)^{2},(s+1)^{2}\right\}$, and $K_{p a}=E_{n-2}$. It can be seen that the signs of principal minors of $K_{p a}$ would change if the signs of $b_{d 3 e i}$ and $b_{d 6 r i}$ changed. The physical meaning of such sign changes is that the elevators and rudders generate totally opposite angular accelerations after damage. Such a situation is unlikely to occur unless the shift of center of gravity is very large. For certain damage scenarios, such as loss of wing-tip and loss of stabilizer, the shift of center of gravity is small, the signs of $b_{d 3 e i}$ and $b_{d 6 r i}$ remain the same with 
the ones before damage, and the coupled terms in $K_{p a}$ are not large enough to change the sign of the second leading principal minor. That is $\operatorname{sign}\left(\Delta_{1}\right)=-1, \operatorname{sign}\left(\Delta_{2}\right)=1$ after damage occurs.

Case II: damage occurs before failures happen. When damage occurs first, the matrices $A$ and $B$ change to the damaged values given as (5.2.9) and $B_{a}=B H$. So, we have the coefficients $E_{n-1}=0$ and

$$
E_{n-2}=C A B_{a}=\left[\begin{array}{cc}
\sum_{i=1}^{4} b_{d 3 e i} & b_{d 3 r 1}+b_{d 3 r 2} \\
\frac{1}{\cos \theta_{0}} \sum_{i=1}^{4} b_{d 6 e i} & \frac{1}{\cos \theta_{0}}\left(b_{d 6 r 1}+b_{d 6 r 2}\right)
\end{array}\right] .
$$

Then, we can choose $\xi_{m}(s)$ as $\xi_{m}(s)=\operatorname{diag}\left\{(s+1)^{2},(s+1)^{2}\right\}$, and $K_{p a}=E_{n-2}$. Similar to the analysis in case I, for certain damage scenarios, such as loss of wing-tip and loss of stabilizer, the signs are invariant: $\operatorname{sign}\left(\Delta_{1}\right)=-1, \operatorname{sign}\left(\Delta_{2}\right)=1$.

Then, after failures happen, some of the remaining actuators are locked. So the matrix $B_{a}$ with certain failure patterns $\left\{j_{1}, \ldots, j_{q_{e}}\right\}$ and $\left\{l_{1}, l_{q_{r}}\right\}$ becomes (5.2.10). The coefficients are calculated as $E_{n-1}=0$ and $E_{n-2}=C A B_{a}$ as in (5.2.13). We can conclude that the interactor matrix is $\xi_{m}(s)=\operatorname{diag}\left\{(s+1)^{2},(s+1)^{2}\right\}$, and the signs are invariant: $\operatorname{sign}\left(\Delta_{1}\right)=-1, \operatorname{sign}\left(\Delta_{2}\right)=1$.

Summary. For these two patterns, the interactor matrix $\xi_{m}(s)$ is invariant before and after hazardous conditions occur, and the signs of leading principal minors of $K_{p a}$ are invariant as well, for certain damage scenarios, such as wing-tip off and stabilizer off, which may cause small shift of the center of gravity. These invariance properties will be further verified by the GTM simulation study.

\subsection{Adaptive Control Scheme}

For the control design of the linear system (5.1.6), the invariant interactor matrix $\xi_{m}(s)$ for all possible failure and damage patterns ensures that we can choose a common reference system $W_{m}(s)=\xi_{m}^{-1}(s)$. To achieve the desired output tracking and 
closed-loop system stability before and after hazardous conditions occur, we develop the following output feedback adaptive control scheme.

The controller $v_{0}(t)$ for the system (5.1.6) is chosen as

$$
v_{0}(t)=\Theta_{1}^{T}(t) \omega_{1}(t)+\Theta_{2}^{T}(t) \omega_{2}(t)+\Theta_{20}(t) y(t)+\Theta_{3}(t) r(t)+\Theta_{4}(t)
$$

where $\omega_{1}(t)=F(s)\left[v_{0}\right](t), \omega_{2}(t)=F(s)[y](t), F(s)=\frac{A_{0}(s)}{\Lambda(s)}, A_{0}(s)=\left[I, s I, \ldots, s^{\bar{\nu}_{0}-2} I\right]^{T}$, $\Lambda(s)$ is a monic stable polynomial of degree $\bar{\nu}_{0}-1$ with the upper bound $\bar{\nu}_{0}$ on the observability indices of $G_{a}(s)$, and $\Theta_{1}(t), \Theta_{2}(t), \Theta_{20}(t), \Theta_{3}(t)$, and $\Theta_{4}(t)$ are the estimates of the nominal piecewise parameters $\Theta_{1}^{*}, \Theta_{2}^{*}, \Theta_{20}^{*}, \Theta_{3}^{*}$, and $\Theta_{4}^{*}$. In particular, the term $\Theta_{4}(t)$ is for compensation of the actuator failures and the unknown dynamics offset $f_{0}$. The existence of the nominal parameters $\Theta_{1}^{*}, \Theta_{2}^{*}, \Theta_{20}^{*}, \Theta_{3}^{*}$ and $\Theta_{4}^{*}$ are guaranteed by the following plant-model matching condition.

Lemma 5.3.1. There exist $\Theta_{1}^{*}, \Theta_{2}^{*}, \Theta_{20}^{*}, \Theta_{3}^{*}$ and $\Theta_{4}^{*}$, such that, when $\Theta_{1}(t)=\Theta_{1}^{*}$, $\Theta_{2}(t)=\Theta_{2}^{*}, \Theta_{20}(t)=\Theta_{20}^{*}, \Theta_{3}(t)=\Theta_{3}^{*}$, and $\Theta_{4}(t)=\Theta_{4}^{*}$, the controller (5.3.1) ensures signal boundedness and output tracking $\lim _{t \rightarrow \infty}\left(y-y_{m}\right)=0$ for the system (5.1.6).

Proof: Substituting the system $y(t)=G_{a}(s)\left[v_{0}^{*}\right](t)+\bar{y}(t)$ in the nominal control law:

$$
v_{0}^{*}(t)=\Theta_{1}^{* T} \omega_{1}(t)+\Theta_{2}^{* T} \omega_{2}(t)+\Theta_{20}^{*} y(t)+\Theta_{3}^{*} r(t)+\Theta_{4}^{*},
$$

we have

$$
\begin{aligned}
v_{0}^{*}(t)= & \left(I-\Theta_{1}^{* T} F(s)-\Theta_{2}^{* T} F(s) G_{a}(s)-\Theta_{20}^{*} G_{a}(s)\right)^{-1} \\
& \times\left(\Theta_{2}^{* T} F(s)[\bar{y}](t)+\Theta_{20}^{*} \bar{y}(t)+\Theta_{3}^{*} r(t)+\Theta_{4}^{*}\right) .
\end{aligned}
$$

As shown in Section 2.4.2, there exist $\Theta_{1}^{*}, \Theta_{2}^{*}, \Theta_{20}^{*}$, and $\Theta_{3}^{*}=K_{p a}^{-1}$, such that

$$
I-\Theta_{1}^{* T} F(s)-\left(\Theta_{2}^{* T} F(s)+\Theta_{20}^{*}\right) G_{a}(s)=\Theta_{3}^{*} W_{m}^{-1}(s) G_{a}(s) .
$$


Then, in view of (5.3.3), we have

$$
\begin{aligned}
y & =W_{m}(s)[r](t)+W_{m}(s) K_{p a}\left(\left(I-\Theta_{1}^{* T} F(s)\right) G_{a}^{-1}(s)[\bar{y}](t)+\Theta_{4}^{*}\right) \\
& \triangleq W_{m}(s)[r](t)+f_{p}(t) .
\end{aligned}
$$

From the reference (5.1.7), we have $e(t)=y(t)-y_{m}(t)$ as

$$
\begin{aligned}
e(t)= & W_{m}(s) K_{p a}\left[\frac{\Lambda(s) I-\Theta_{1}^{* T} A_{0}(s)}{\Lambda(s)} Z_{l a}^{-1}(s) Z_{l b}(s)[\bar{u}]\right. \\
& \left.+\frac{\Lambda(s) I-\Theta_{1}^{* T} A_{0}(s)}{\Lambda(s)} Z_{l a}^{-1}(s) Z_{f}(s)\left[h_{f}\right]+\Theta_{4}^{*}\right](t)
\end{aligned}
$$

From (A3) where $Z_{l a}^{-1} Z_{l b}(s)$ and $Z_{l a}^{-1} Z_{f}(s)$ are proper, it can be concluded that $\frac{\Lambda(s) I-\Theta_{1}^{* T} A_{0}(s)}{\Lambda(s)} Z_{l a}^{-1}(s) Z_{l b}(s)$ and $\frac{\Lambda(s) I-\Theta_{1}^{* T} A_{0}(s)}{\Lambda(s)} Z_{l a}^{-1}(s) Z_{f}(s)$ are proper. Since $\bar{u}(t)$ and $h_{f}(t)$ are step signals, $W_{m}(s)$ and $\Lambda(s)$ are stable, and $G_{a}(s)$ is minimum phase, there exists a $\Theta_{4}^{*}$ to make $\lim _{t \rightarrow \infty} e(t)=0$.

Remark 5.3.1. The parameters $\Theta_{1}^{*}, \Theta_{2}^{*}, \Theta_{20}^{*}, \Theta_{3}^{*}$ and $\Theta_{4}^{*}$ are piecewise constant $m a-$ trices for different damage and failure patterns $\left(A_{k}, B_{k}, C_{k}, f_{0 k}, \sigma_{k}\right), k=1,2, \ldots, N$. Since the interactor matrix $\xi_{m}(s)$ is invariant under different damage and failure conditions, there exists a common reference system $W_{m}(s)=\xi_{m}^{-1}(s)$ for all damage and failure conditions.

Since the parameters are unknown due to uncertain damage and failures, the adaptive control law (5.3.1) is employed.

Tracking error equation. Operating both sides of (5.3.4) on $v_{0}(t)$ and from the system transfer function (5.1.6), we obtain

$$
\begin{aligned}
v_{0}(t)= & \Theta_{1}^{* T} \omega_{1}(t)+\Theta_{2}^{* T} \omega_{2}(t)+\Theta_{20}^{*} y(t)-\Theta_{2}^{* T} F(s)[\bar{y}](t) \\
& -\Theta_{20}^{*} \bar{y}(t)+\Theta_{3}^{*} \xi_{m}(s)[y](t)-\Theta_{3}^{*} \xi_{m}(s)[\bar{y}](t)
\end{aligned}
$$


Substituting (5.3.1) in (5.3.6), we obtain the tracking error as

$$
e(t)=y(t)-y_{m}(t)=W_{m}(s) K_{p a}\left[\widetilde{\Theta}^{T} \omega\right](t)+f_{p}(t),
$$

where $\widetilde{\Theta}=\Theta-\Theta^{*}, \Theta=\left[\Theta_{1}^{T}, \Theta_{2}^{T}, \Theta_{20}, \Theta_{3}, \Theta_{4}\right]^{T}, \Theta^{*}=\left[\Theta_{1}^{* T}, \Theta_{2}^{* T}, \Theta_{20}^{*}, \Theta_{3}^{*}, \Theta_{4}^{*}\right]^{T}$, and $\omega=\left[\omega_{1}^{T}, \omega_{2}^{T}, y^{T}, r^{T}, 1\right]^{T}$.

To deal with the uncertainty of $K_{p a}$, we use its LDS decomposition (2.3.5): $K_{p a}=L_{s} D_{s} S$, where $S=S^{T}>0, L_{s}$ is a unit lower triangular matrix, and $D_{s}=\operatorname{diag}\left\{\operatorname{sign}\left[\Delta_{1}\right] \gamma_{1}, \ldots, \operatorname{sign}\left[\frac{\Delta_{M}}{\Delta_{M-1}}\right] \gamma_{M}\right\}$ with arbitrarily chosen $\gamma_{i}>0, i=1, \ldots, M$ [84]. Since the signs of the leading principal minors $\Delta_{i}, i=1,2, \ldots, M$, are invariant, we can choose a uniform $D_{s}$ for the possible damage and failure patterns as a gain matrix which will be used in the adaptive laws.

Ignoring the decaying term $f_{p}$, and substituting the LDS decompensation in (5.3.7) and operating both sides of (5.3.7) by $h(s) I_{M}$, where $h(s)=1 / f_{h}(s)$ with $f_{h}(s)$ being a stable and monic polynomial of degree equals to the degree of $\xi_{m}(s)$, we have

$$
L_{s}^{-1} \xi_{m}(s) h(s)[e](t)=D_{s} S h(s)\left[\tilde{\Theta}^{T} \omega\right](t) .
$$

To parameterize the unknown matrix $L_{s}$, we introduce $\Theta_{0}^{*}=L_{s}^{-1}-I=\left\{\theta_{i j}^{*}\right\}$, where $\theta_{i j}^{*}=0$ for $i=1,2, \ldots, M$ and $j \geq i$. Then we have

$$
\bar{e}(t)+\left[0, \theta_{2}^{* T} \eta_{2}(t), \ldots, \theta_{M}^{* T} \eta_{M}(t)\right]^{T}=D_{s} S h(s)\left[\widetilde{\Theta}^{T} \omega\right](t),
$$

where $\bar{e}(t)=\xi_{m}(s) h(s)[e](t)=\left[\bar{e}_{1}(t), \ldots, \bar{e}_{M}(t)\right]^{T}, \eta_{i}(t)=\left[\bar{e}_{1}(t), \ldots, \bar{e}_{i-1}(t)\right]^{T}, \theta_{i}^{*}=$ $\left[\theta_{i 1}^{*}, \ldots, \theta_{i i-1}^{*}\right]^{T}, i=2, \ldots, M$.

Estimation error. We introduce the estimation error

$$
\epsilon(t)=\left[0, \theta_{2}^{T}(t) \eta_{2}(t), \ldots, \theta_{M}^{T}(t) \eta_{M}(t)\right]^{T}+\Psi(t) \xi(t)+\bar{e}(t),
$$

where $\theta_{i}(t), i=2,3, \ldots, M$ are the estimates of $\theta_{i}^{*}$, and $\Psi(t)$ is the estimate of $\Psi^{*}=$ $D_{s} S$, and

$$
\xi(t)=\Theta^{T}(t) \zeta(t)-h(s)\left[\Theta^{T} \omega\right](t), \zeta(t)=h(s)[\omega](t) .
$$


From (5.3.9)-(5.3.11), we can derive that

$$
\epsilon(t)=\left[0, \tilde{\theta}_{2}^{T}(t) \eta_{2}(t), \tilde{\theta}_{3}^{T}(t) \eta_{3}(t), \ldots, \tilde{\theta}_{M}^{T}(t) \eta_{M}(t)\right]^{T}+D_{s} S \tilde{\Theta}^{T}(t) \zeta(t)+\tilde{\Psi}(t) \xi(t)
$$

where $\tilde{\theta}_{i}(t)=\theta_{i}(t)-\theta_{i}^{*}, i=2,3, \ldots, M$ and $\tilde{\Psi}(t)=\Psi(t)-\Psi^{*}$.

Adaptive laws. With the estimation error model (5.3.12), we choose

$$
\begin{aligned}
\dot{\theta}_{i}(t) & =-\frac{\Gamma_{\theta i} \epsilon_{i}(t) \eta_{i}(t)}{m^{2}(t)}, i=2,3, \ldots, M \\
\dot{\Theta}^{T}(t) & =-\frac{D_{s} \epsilon(t) \zeta^{T}(t)}{m^{2}(t)} \\
\dot{\Psi}(t) & =-\frac{\Gamma \epsilon(t) \xi^{T}(t)}{m^{2}(t)}
\end{aligned}
$$

where the signal $\epsilon(t)=\left[\epsilon_{1}(t), \epsilon_{2}(t), \ldots, \epsilon_{M}(t)\right]^{T}$ is computed from (5.3.10), $\Gamma_{\theta i}=\Gamma_{\theta i}^{T}>$ $0, i=2,3, \ldots, M$, and $\Gamma=\Gamma^{T}>0$ are adaptation gain matrices, and

$$
m(t)=\left(1+\zeta^{T}(t) \zeta(t)+\xi^{T}(t) \xi(t)+\sum_{i=2}^{M} \eta_{i}^{T}(t) \eta_{i}(t)\right)^{1 / 2} .
$$

From the adaptive laws (5.3.13)-(5.3.15), the stability properties can be derived.

Lemma 5.3.2. The adaptive laws (5.3.13)-(5.3.15) ensure that

(i) $\theta_{i}(t) \in L^{\infty}, i=2,3, \ldots, M, \Theta(t) \in L^{\infty}, \Psi(t) \in L^{\infty}$, and $\epsilon(t) / m(t) \in L^{2} \cap L^{\infty}$;

(ii) $\dot{\theta}_{i}(t) \in L^{2} \cap L^{\infty}, i=2,3, \ldots, M, \dot{\Theta}(t) \in L^{2} \cap L^{\infty}$, and $\dot{\Psi}(t) \in L^{2} \cap L^{\infty}$.

Proof: Consider a positive definite function

$$
V=\frac{1}{2}\left(\sum_{i=2}^{m} \tilde{\theta}_{i}^{T} \Gamma_{\theta i}^{-1} \tilde{\theta}_{i}+\operatorname{tr}\left[\tilde{\Psi}^{T} \Gamma^{-1} \tilde{\Psi}\right]+\operatorname{tr}\left[\tilde{\Theta} S \tilde{\Theta}^{T}\right]\right)
$$

which is continuous at each interval $\left(t_{k-1}, t_{k}\right), k=1,2, \ldots, N$, with $t_{0}=0$ and $t_{N}=$ $\infty$, and has a finite jump at $t_{k}, k=1,2, \ldots, N-1$, i.e.,

$$
V\left(t_{k}^{+}\right)-V\left(t_{k}^{-}\right)<\infty, k=1,2, \ldots, N-1
$$


From the adaptive laws (5.3.13)-(5.3.15), the time-derivative of $V$ in each $\left(t_{k-1}, t_{k}\right), k=$ $1,2, \ldots, N$ is obtained as

$$
\dot{V}=-\frac{\epsilon^{T}(t) \epsilon(t)}{m^{2}(t)} \leq 0
$$

That is $V\left(t_{k}^{-}\right) \leq V\left(t_{k-1}^{+}\right)$. From (5.3.17) and (5.3.18), $V(t)$ is bounded for $[0, \infty)$, so that $\theta_{i}(t) \in L^{\infty}, i=2,3, \ldots, M, \Theta(t) \in L^{\infty}$, and $\Psi(t) \in L^{\infty}$. Then, integration of both sides of (5.3.18) is given as

$$
\int_{0}^{\infty} \frac{\epsilon^{T}(\tau) \epsilon(\tau)}{m^{2}(\tau)} d \tau=V(0)+\sum_{k=1}^{N-1}\left[V\left(t_{k}^{+}\right)-V\left(t_{k}^{-}\right)\right]-V(\infty) .
$$

Since $V$ is bounded for $[0, \infty)$, it follows that

$$
\int_{0}^{\infty} \frac{\epsilon^{T}(\tau) \epsilon(\tau)}{m^{2}(\tau)} d \tau<\infty
$$

from which $\epsilon(t) / m(t) \in L^{2} \cap L^{\infty}$ can be concluded. Since the normalized signals $\eta_{i}(t) / m(t) \in L^{\infty}, \zeta^{T}(t) / m(t) \in L^{\infty}$ and $\xi^{T}(t) / m(t) \in L^{\infty}$, it can be concluded that $\dot{\theta}_{i}(t) \in L^{2} \cap L^{\infty}, i=2,3, \ldots, M, \dot{\Theta}(t) \in L^{2} \cap L^{\infty}$, and $\dot{\Psi}(t) \in L^{2} \cap L^{\infty}$.

Based on these properties, the desired closed-loop system properties can be proved:

Theorem 5.3.1. The multivariable MRAC scheme with the output feedback control law (5.3.1) updated by the adaptive laws (5.3.13)-(5.3.15), when applied to the plant (5.1.6), guarantees the closed-loop signal boundedness and asymptotic output tracking: $\lim _{t \rightarrow \infty}\left(y(t)-y_{m}(t)\right)=0$.

The first step of this theorem is to express a filtered version of the plant output $y(t)$ in a feedback framework which has a small gain due to the $L^{2}$ properties of $\dot{\Theta}(t), \dot{\theta}_{i}(t)$, and $\epsilon(t) / m(t)$. This step leads to the closed-loop signal boundedness. The asymptotic tracking property follows from the complete parametrization of the error equation (5.3.10), the $L^{2}$ properties, and the signal boundedness. 


\subsection{Application to the NASA GTM}

In this section, the proposed linearization-based MRAC design is applied to the NASA GTM to assess its effectiveness for the nonlinear aircraft system.

Damage and Actuator Failure Scenarios. The GTM Simulink model provides some structural damage scenarios such as rudder off, vertical tail off, left outboard flap off, left wing-tip off, left elevator off, and left stabilizer off. Two damage and failure patterns are considered in this study:

- lock-in-place failures of actuator segments occur first, then the damage with a loss of the entire left stabilizer happens;

- the damage with a loss of the entire left stabilizer happens first, then failures of actuator segments happen.

Design Conditions. Based on the analysis of Section 5.2, to ensure the invariance properties of the infinite zero structure, the operating point for the nonlinear GTM is chosen as a wings-level flight condition $\left(x_{0}, u_{0}\right)$ obtained by trimming the nominal GTM with equivalent airspeed as 90 knots, the output signal is chosen as $y(t)=[\theta(t), \psi(t)]^{T}$, and the control input signal is chosen as $u(t)=\left[d_{e}^{T}(t), d_{r}^{T}(t)\right]^{T}$. Hence, the reference system $\Delta y_{m}(t)=W_{m}(s)[r](t)$ is

$$
W_{m}(s)=\xi_{m}^{-1}(s)=\operatorname{diag}\left\{(s+2)^{-2},(s+1)^{-2}\right\}
$$

with $\Delta y_{m}(t)=y_{m}(t)-C x_{0}$, where $y_{m}(t)$ is the GTM's reference signal. The design conditions (A1), (A2), and (A3) can be verified by the numerical parameter values of the different failure and damage scenarios obtained from the GTM. 


\subsubsection{Control of the Linearized GTM}

Before assessing the performance of the developed linearization-based design on the nonlinear GTM, the adaptive control design will be verified by applying

$$
\Delta v_{0}(t)=\Theta_{1}^{T}(t) \Delta \omega_{1}(t)+\Theta_{2}^{T}(t) \Delta \omega_{2}(t)+\Theta_{20}(t) \Delta y(t)+\Theta_{3}(t) r(t)+\Theta_{4}(t),
$$

to the linearized GTM given as (5.2.4). The simulation results are present as follows.

Fig. 5.1 shows the result of the first damage and failure pattern: after 300 seconds, lock-in-place failures of the left elevator outboard segment, the right elevator inboard segment, and upper rudder segment occur, such that the corresponding actuator perturbation signals are fixed at some uncertain values: $\Delta d_{e l 1}(t)=\Delta \bar{d}_{e l 1}=3 \mathrm{deg}$, $\Delta d_{e r 2}(t)=\Delta \bar{d}_{e r 2}=-6 \mathrm{deg}$, and $\Delta d_{r u}(t)=\Delta \bar{d}_{r u}=4 \mathrm{deg} ;$ then after 600 seconds, the entire left stabilizer is lost. From Fig. 5.1, it can be seen that the linearized GTM's output $\Delta y=[\Delta \theta, \Delta \psi]^{T}$ (solid) tracks the reference $\Delta y_{m}=\left[\Delta \theta_{m}, \Delta \psi_{m}\right]^{T}($ dotted) after failures and damage occur. The second damage and failure pattern is shown in Fig. 5.2: after 300 seconds, the left stabilizer is lost; then after 600 seconds, the right inboard elevator is locked at $\Delta \bar{d}_{e r 2}=-6 \mathrm{deg}$, and after 650 seconds, the upper rudder is locked at $\Delta \bar{d}_{r u}=4 \mathrm{deg}$. From Fig. 5.2, it can be seen that the linearized GTM's output $\Delta y$ (solid) tracks the reference $\Delta y_{m}$ (dotted).

\subsubsection{Control of the Nonlinear GTM}

Since the control law $\Delta v=H \Delta v_{0}(t)$ with $\Delta v_{0}(t)$ in (5.4.2) for the linearized GTM (5.1.3) has been obtained, the control law $v(t)=\Delta v+u_{0}$ can be applied to the nonlinear GTM (5.1.1) to assess the effectiveness of this linearization-based design.

Fig. 5.3, Fig. 5.4 and Fig. 5.5 show the simulation result of the first damage and failure case: after 200 seconds, the left elevator outboard segment is locked at $\bar{d}_{e l 1}=5 \mathrm{deg}$ and after 230 seconds the upper rudder segment is locked at $\bar{d}_{r u}=-2$ deg 

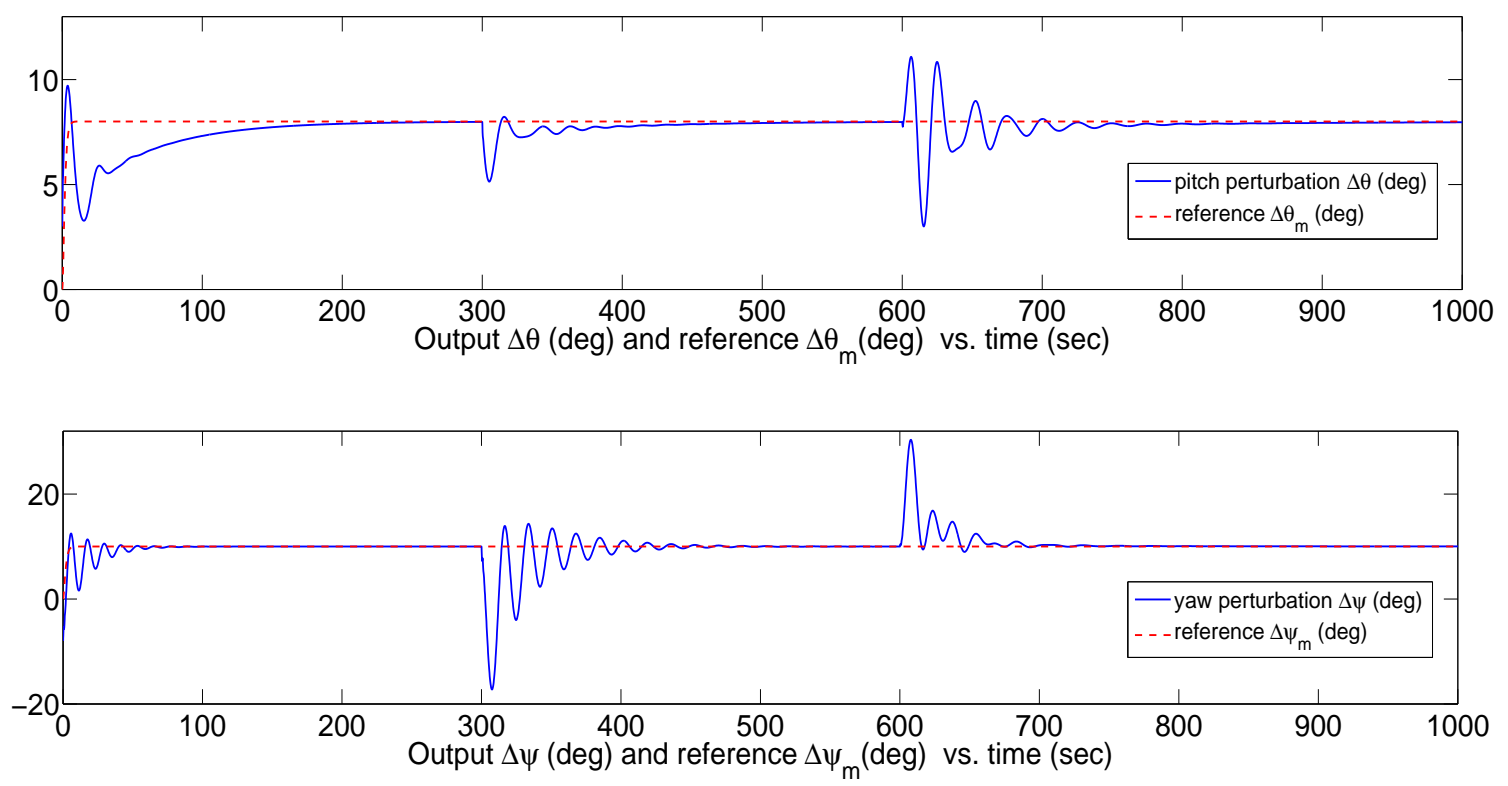

Figure 5.1: Linearized GTM outputs vs. reference signals (case I).
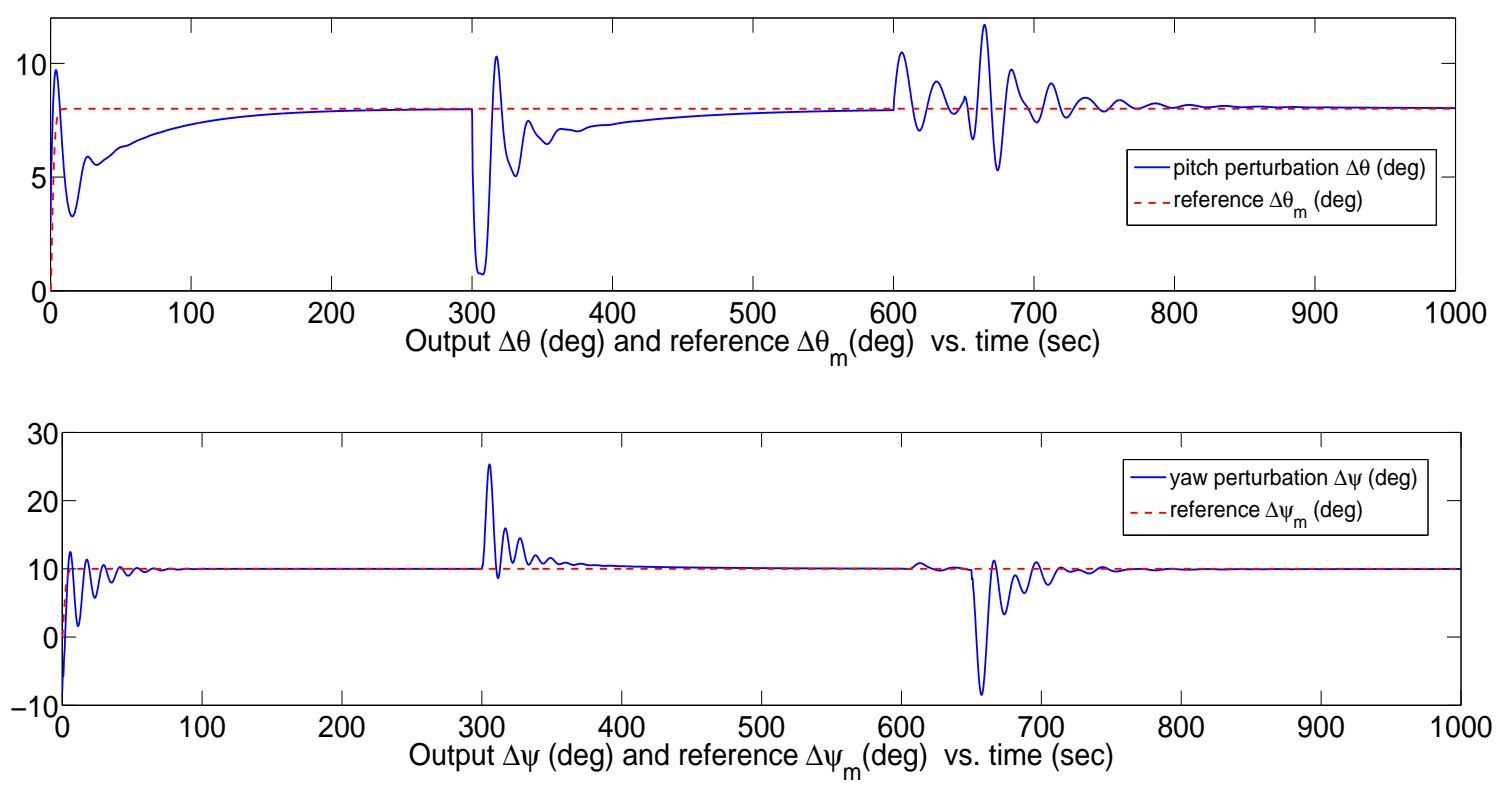

Figure 5.2: Linearized GTM outputs vs. reference signals (case II). 
(as shown in Fig. 5.4 and Fig. 5.5); then after 500 seconds, the entire left stabilizer is lost. From Fig. 5.3, it can be seen that the GTM output $y(t)$ (solid) tracks the reference output signal $y_{m}(t)$ (dotted).
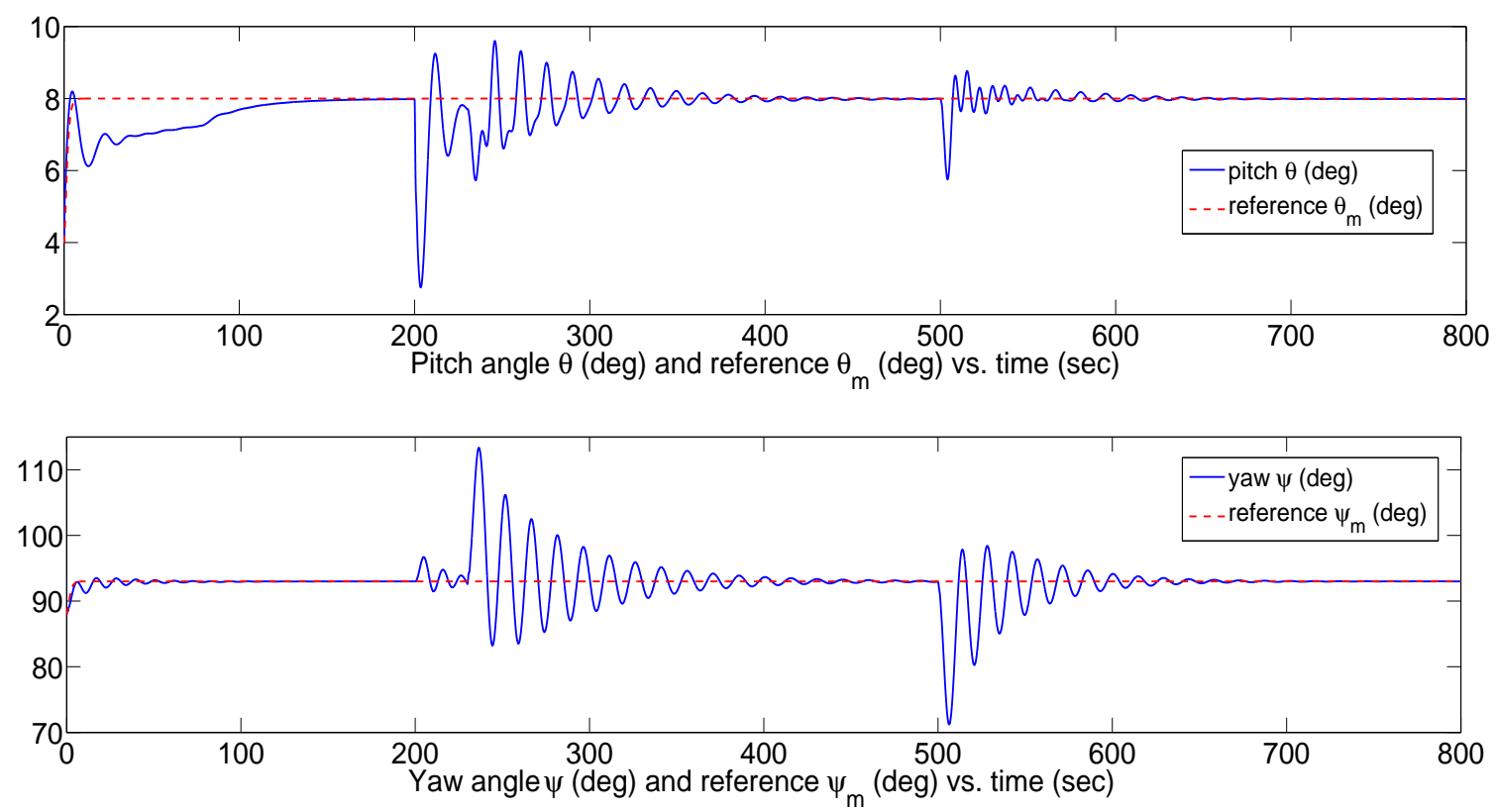

Figure 5.3: GTM outputs (solid) vs. reference outputs (dotted) (case I).

The second damage and failure case is that after 200 seconds, the left stabilizer is lost; then after 400 seconds, the right inboard elevator is locked at $\bar{d}_{e r 2}=-4 \mathrm{deg}$, and after 420 seconds, the lower rudder is locked at $\bar{d}_{r l}=2$ deg. Fig. 5.6 shows the output tracking performance and Fig. 5.7 and Fig. 5.8 illustrate the responses of the control surface segments.

Thus, it can be concluded that this linearization-based design is applicable for the nonlinear GTM with damage and actuator failures around a neighborhood of the operating point $\left(x_{0}, u_{0}\right)$.

Remark 5.4.1. During the transient period after the hazardous condition occurs, the adaptive controller automatically adjusts its parameters to accommodate the large 

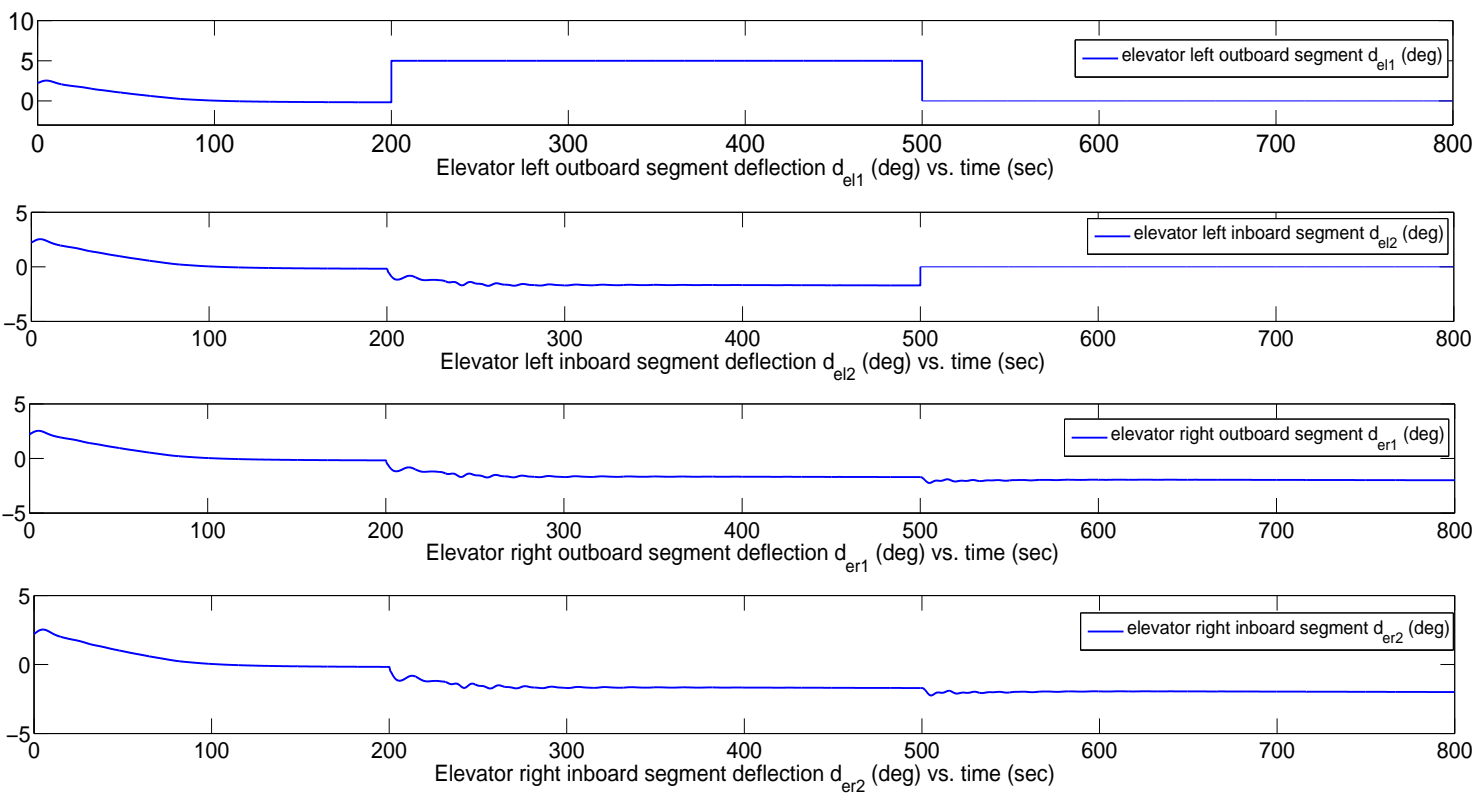

Figure 5.4: Deflections of four elevator segments (case I).
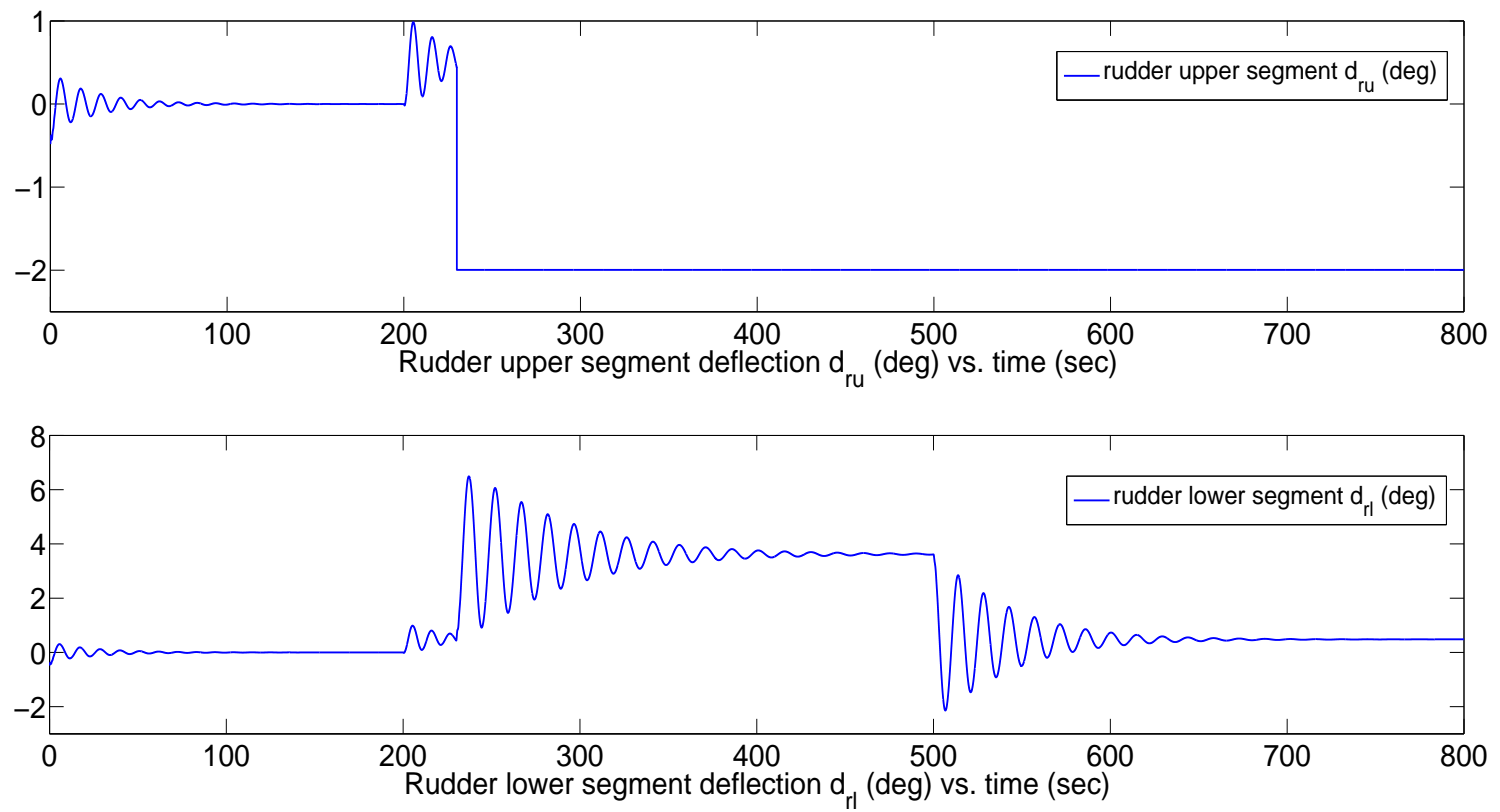

Figure 5.5: Deflections of two rudder segments (case I). 

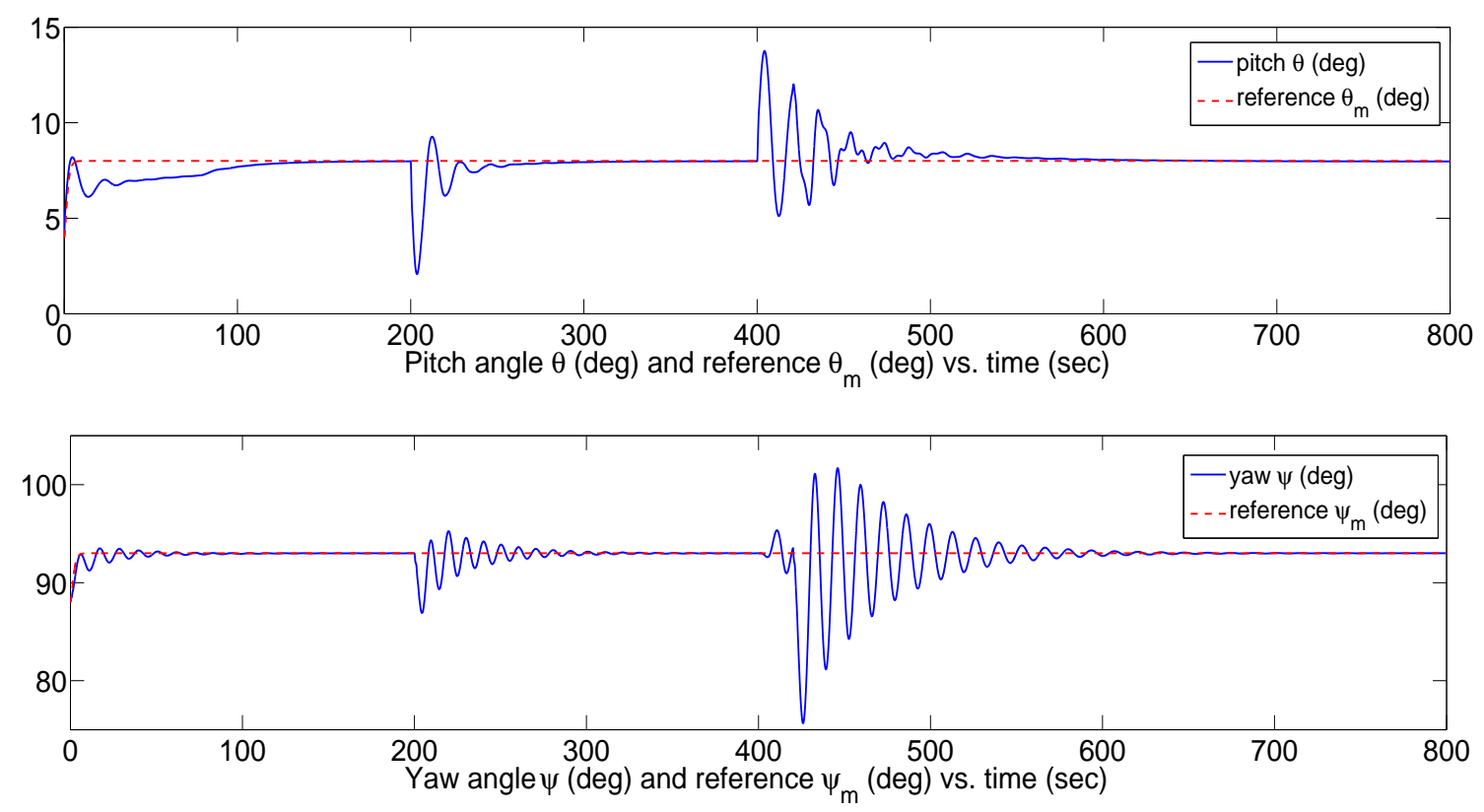

Figure 5.6: GTM outputs (solid) vs. reference outputs (dotted) (case II).
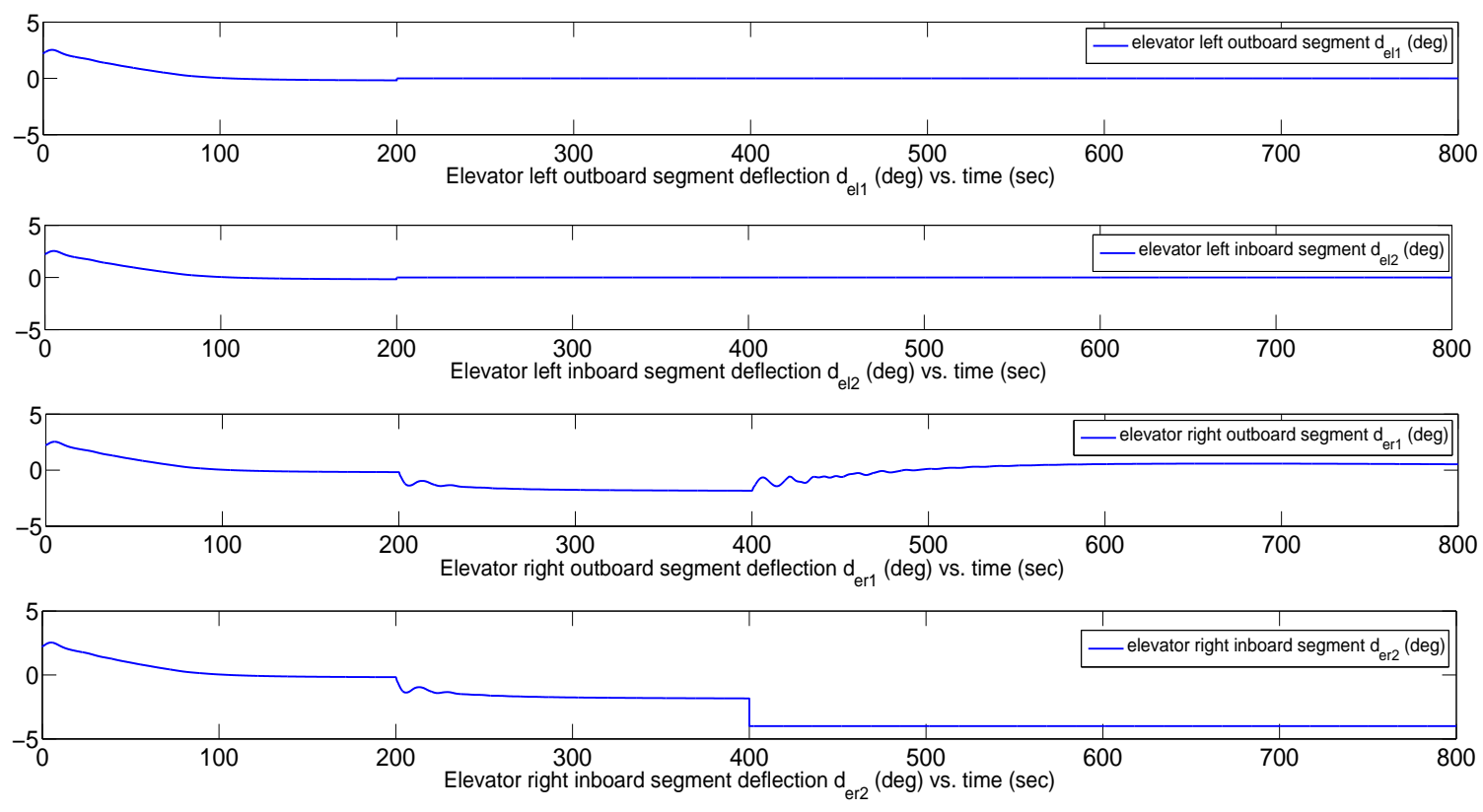

Figure 5.7: Deflections of four elevator segments (case II). 

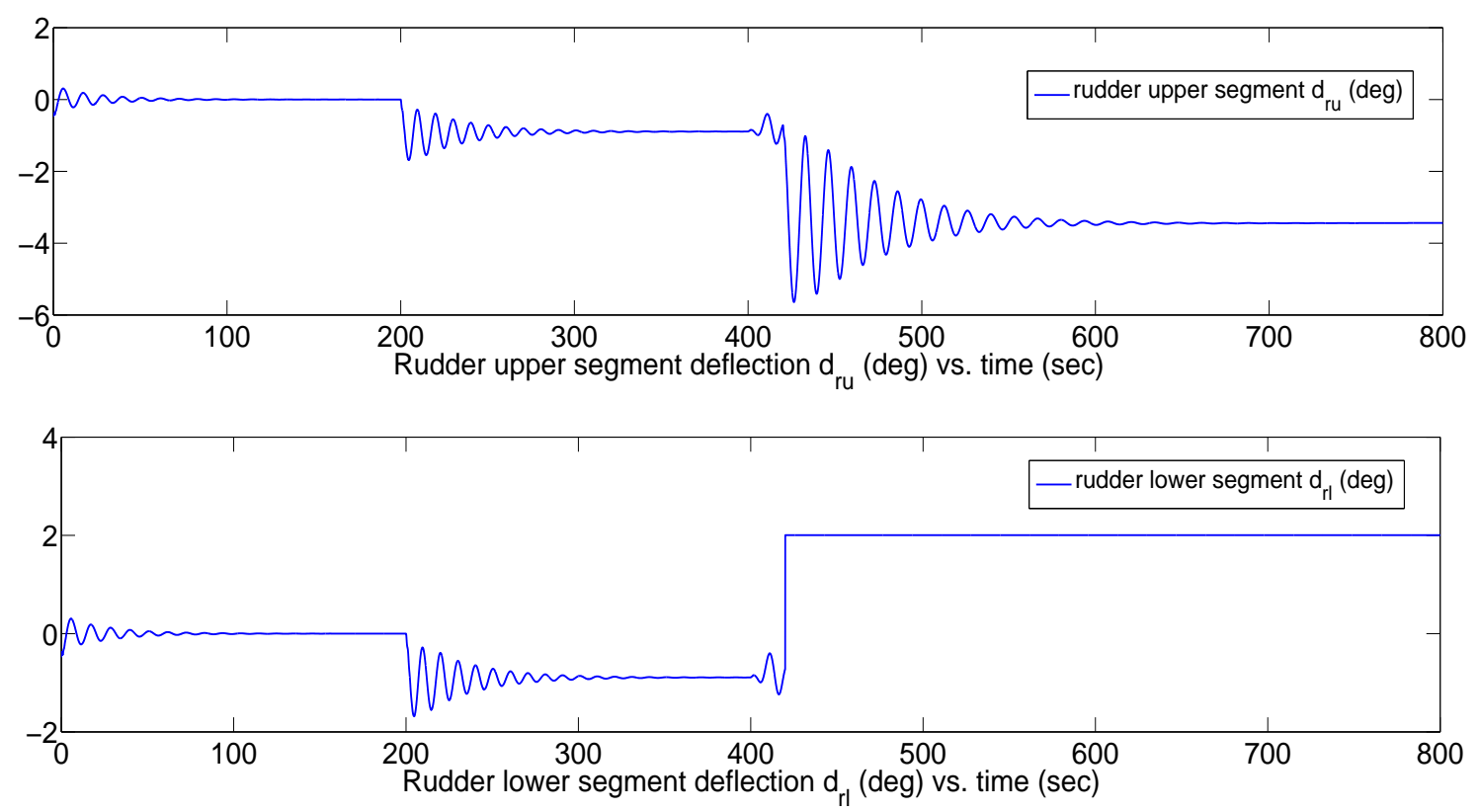

Figure 5.8: Deflections of two rudder segments (case II).

unknown system dynamics variations due to damage and to reject the uncertain disturbances due to actuator failures. Hence, before the adaptive controller can make the damaged or failed system go to steady state, i.e., the output tracking errors converge to zero, there may exist oscillations (as shown in the simulation output responses) introduced by the large system uncertainties.

\section{Summary}

In this chapter, the modeling and control of aircraft under simultaneous failure and damage conditions have been studied and evaluated. An extensive generic analysis of the linearized aircraft models under failures and damage has been conducted. It has been shown that two essential conditions for multivariable model reference adaptive control designs, namely, the interactor matrix and the signs of leading principal minors of the high frequency gain matrix, can remain invariant under realistic failure 
and damage conditions for the linearized aircraft system. A multivariable model reference adaptive control scheme has been developed for aircraft under the hazardous conditions, without the need of explicit detection of actuator failures and structural damage. The stability analysis has shown that the proposed adaptive control scheme is capable of ensuring closed-loop stability and asymptotic output tracking in the presence of uncertain failures and damage. A thorough evaluation study for the nonlinear NASA generic transport model has been conducted to show the effectiveness of the proposed adaptive compensation scheme. 


\section{Chapter 6}

\section{Adaptive Sensor Uncertainty Compensation for MIMO Systems}

This chapter develops a multivariable MRAC scheme for linear systems with parameterized sensor uncertainties. For sensor failure detection, a typical method is based on neural networks and analytical sensor redundancy, such as $[16,28,66,94]$. Specific applications have also been developed in the field of flight control, such as $[14,40,67,68]$. They all need to detect and isolate the failed sensors first, before the sensor signals can be used in feedback control. Using the feedback control based sensor uncertainty compensation approach (to be developed in this chapter), an adaptive sensor failure compensation scheme can be constructed without explicit failure detection, where

- sensor uncertainty compensators are designed to estimate actual signals;

- state feedback controller is constructed using sensor compensator signals;

- stable adaptive laws are derived to update the controller parameters to achieve system stability and output tracking performance; and

- simulation results on a linear aircraft model show the desired performance. 


\subsection{Problem Statement}

Consider a MIMO linear time-invariant system

$$
\dot{x}(t)=A x(t)+B u(t), y(t)=C x(t)
$$

with $A \in R^{n \times n}, B \in R^{n \times M}$ and $C \in R^{M \times n}$ being unknown constant parameter matrices, and $x(t) \in R^{n}, u(t) \in R^{M}$ and $y(t) \in R^{M}$ being the system state, input and output vector signals.

For feedback control, we need sensors to obtain the state signal $x(t)$ or the output signal $y(t)$ to construct the controller signal $u(t)$. Often sensors may have uncertainties so that the senors signals are not the actual measured signals, which can result in deterioration of the performance of control systems. For sensor uncertainty compensation, we also need some sensor uncertainty models.

Sensor uncertainty model. A sensor uncertainty model may be represented by

$$
z(t)=S(\varphi(t))=f_{s}(\varphi(t))+d_{s}(t)
$$

where $\varphi(t)$ is the actual signal, and $d_{s}(t)$ is a noise signal.

In this chapter, we will consider the static and parameterized sensor uncertainty characteristic described as

$$
z(t)=k_{s} \varphi(t)+\sum_{i=1}^{q} b_{i} f_{i}(t)
$$

where $k_{s}$ and $b_{i}, i=1,2, \ldots, q$, are unknown constants, and $f_{i}(t), i=1,2, \ldots, q$, are known bounded signals.

The sensor model (6.1.3) can represent a class of practical sensor uncertainties such as sensor gain variations and measurement errors, as well as sensor failures when a set of redundant sensors are used. We may use several sensors to measure 
the same signal $\varphi(t)$, and take the weighted sum of the sensors' output signals $z_{i}(t)$ as $z(t)=\sum_{i=1}^{m} \alpha_{i} z_{i}(t)$, where $\alpha_{i}>0, i=1, \ldots, m$, such that $\sum_{i=1}^{m} \alpha_{i}=1$. When there is no failure for all the sensors, the summed sensor signal $z(t)$ is the exact measured signal $\varphi(t)$. When there are some sensors failed, e.g., the $i_{1}, i_{2}, \ldots, i_{p}$ th sensors fail and generate some random signals $\bar{z}_{i}(t)$, the summed sensor signal is $z(t)=\alpha_{s} \varphi(t)+d_{s}(t)$, where $\alpha_{s}=\sum_{i \neq i_{1}, i_{2}, \ldots, i_{p}} \alpha_{i}$, and $d_{s}(t)=\sum_{i=i_{1}, i_{2}, \ldots, i_{p}} \alpha_{i} \bar{z}_{i}(t)$. Since the indexes $i_{1}, i_{2}, \ldots, i_{p}$ and the signals $\bar{z}_{i}(t)$ for $i_{1}, i_{2}, \ldots, i_{p}$ are unknown, we may express $d_{s}(t)=\sum_{i=1}^{m} \beta_{i} \bar{z}_{i}(t)$, where some of $\beta_{i}$ are zero (for the unfailed sensors) while others are $\alpha_{i}$. Thus, the sensor failures can be characterized by the sensor uncertainty model (6.1.3) using redundant sensors.

Sensor uncertainty compensation problem. In this chapter, we will use state feedback control for output tracking. There exist two sets of sensors: state sensors and output sensors. We will consider the systems with both state sensor uncertainties and output sensor uncertainties.

As described in Fig. 6.1 where sensors are used to measure the state vector $x(t)$, the state sensor vector signal is $z_{x}(t)$. Since the state sensors have uncertainties, the output signal $z_{x}(t)=\left[z_{x 1}(t), z_{x 2}(t), \ldots, z_{x n}(t)\right]^{T}$ is not equal to the actual state signal $x(t)=\left[x_{1}(t), x_{2}(t), \ldots, x_{n}(t)\right]^{T}$. With (6.1.3), the output signal $z_{x}(t)=\left[z_{x 1}(t), z_{x 2}(t), \ldots, z_{x n}(t)\right]^{T}$ of the state sensors can be described as

$$
z_{x i}(t)=k_{x i} x_{i}(t)+\sum_{j=1}^{q_{i}} b_{x i j} f_{x i j}(t), i=1,2, \ldots, n,
$$

where $k_{x i}$ and $b_{x i j}, i=1,2, \ldots, n, j=1,2, \ldots, q_{i}$, are unknown constants, and $f_{x i j}(t), i=$ $1, \ldots, n, j=1, \ldots, q_{i}$, are known bounded signals.

Since the parameters $k_{x i}$ and $b_{x i j}, i=1,2, \ldots, n, j=1,2, \ldots, q_{i}$, in (6.1.4) are unknown, we cannot retrieve the state signals $x_{i}(t), i=1,2, \ldots, n$, from (6.1.4) directly. To overcome this difficulty, we propose to use sensor compensators, which are also 
shown in Fig.6.1, to derive a compensation vector signal $\hat{x}(t)=\left[\hat{x}_{1}(t), \hat{x}_{2}(t), \ldots, \hat{x}_{n}(t)\right]^{T}$ from $z_{x}(t)$ to estimate the actual state signal $x(t)$. Then, we can use the estimate signal $\hat{x}(t)$ to construct a state feedback controller, instead of the unavailable actual state signal $x(t)$.

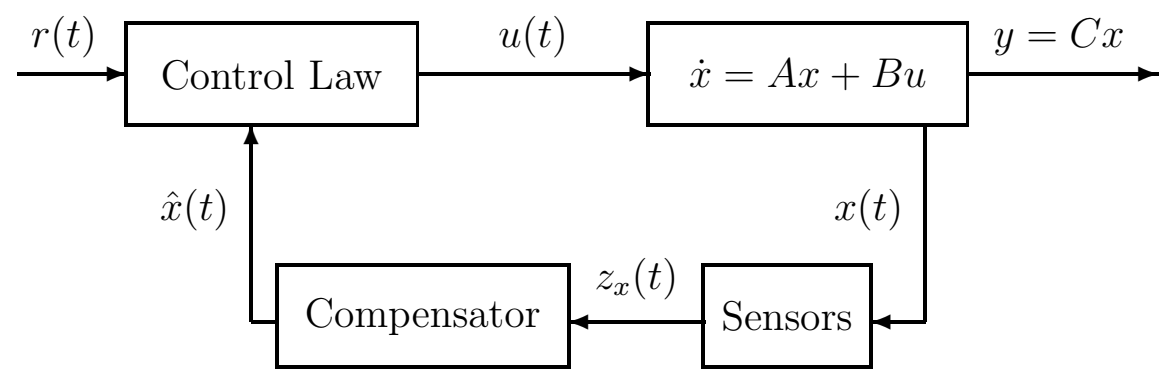

Figure 6.1: Control system with sensor uncertainties.

Control objective. The control objective is to construct a feedback control law for $u(t)$ (as shown in Figure 6.1) using the state sensor compensation signal $\hat{x}(t)$, for the plant (6.1.1) with state and output sensor uncertainties, to make all the closedloop signals bounded and the plant output $y(t)$ track a given reference vector signal $y_{m}(t) \in R^{M}$ generated from the reference model system

$$
y_{m}(t)=W_{m}(s)[r](t), W_{m}(s)=\xi_{m}^{-1}(s)
$$

where $r(t) \in R^{M}$ is a bounded reference input signal.

Assumptions. To design a multivariable state feedback MRAC scheme, we make the standard assumptions: (A1) all zeros of $G(s)$ have negative real parts; (A2) $G(s)$ is strictly proper, has full rank and its modified left interactor matrix $\xi_{m}(s)$ is known; (A3) there exists a known matrix $S_{p} \in R^{M \times M}$ such that $\Gamma_{p}=K_{p}^{T} S_{p}^{-1}=\Gamma_{p}^{T}>0$, where $K_{p}=\lim _{s \rightarrow \infty} \xi_{m}(s) G(s)$ is the high frequency gain matrix of $G(s) ;(\mathrm{A} 4)(A, B)$ is controllable and $(A, C)$ is observable. 
When the output sensors do not have uncertainties, the actual output tracking error $e(t)=y(t)-y_{m}(t)$ can be obtained. The adaptive compensation design can be simplified. However, in this chapter, we mainly focus on the case when output sensors have uncertainties, that is, the actual output signal $y(t)$ cannot be obtained. Thus, we need to generate an output compensation signal $\hat{y}(t)$ to estimate $y(t)$. Then, we will develop an adaptive control scheme based on the compensation tracking error $\hat{e}(t)=\hat{y}(t)-y_{m}(t)$.

\subsection{Adaptive Compensation Scheme}

Since the state sensors have uncertainties, we need to design the state sensor uncertainty compensators first.

State sensor compensator design. From (6.1.4), we can retrieve the state signals $x_{i}(t), i=1, \ldots, n$, by using the inverse $S I_{x i}(\cdot)$ of $S_{x i}(\cdot)$, which are given by

$$
x_{i}(t)=S I_{x i}\left(z_{x i}\right)=\theta_{x i}^{* T} \psi_{x i}(t)
$$

where $\theta_{x i}^{*}=\left[\theta_{k x i}^{*}, \theta_{b x i 1}^{*}, \ldots, \theta_{b x i q_{i}}^{*}\right]^{T}, \psi_{x i}(t)=\left[z_{x i}(t), f_{x i 1}(t), \ldots, f_{x i q_{i}}(t)\right]^{T}$, with $\theta_{k x i}^{*}=$ $1 / k_{x i}, \theta_{b x i j}^{*}=-b_{x i j} / k_{x i}, i=1,2, \ldots, n, j=1,2, \ldots, q_{i}$ being unknown constant parameters. Thus, the state vector $x(t)$ is expressed as

$$
x(t)=\left[x_{1}(t), \ldots, x_{n}(t)\right]^{T}=\Theta_{x}^{* T} \psi_{x}(t),
$$

where $\Theta_{x}^{* T}=\operatorname{diag}\left\{\theta_{x 1}^{* T}, \theta_{x 2}^{* T}, \ldots, \theta_{x n}^{* T}\right\}, \psi_{x}(t)=\left[\psi_{x 1}^{T}(t), \ldots, \psi_{x n}^{T}(t)\right]^{T}$.

Since the parameters of (6.2.1) are unknown, we can not obtain the actual state signal $x(t)$. To compensate such sensor uncertainties, we use the adaptive inverse compensator $\widehat{S I}_{x i}(\cdot)$ to generate the estimates of the unaccessible state signals $x_{i}(t), i=$ $1, \ldots, n$, which are given as

$$
\hat{x}_{i}(t)=\widehat{S I}_{x i}\left(z_{x i}\right)=\theta_{x i}^{T}(t) \psi_{x i}(t),
$$


where $\theta_{x i}(t)=\left[\theta_{k x i}(t), \theta_{b x i 1}(t), \ldots, \theta_{b x i q_{i}}(t)\right]^{T}, i=1,2, \ldots, n$, are the adaptive estimates of the unknown constant parameters $\theta_{x i}^{*}$. The estimate of the state vector signal $x(t)$ can be expressed as

$$
\hat{x}(t)=\left[\hat{x}_{1}(t), \ldots, \hat{x}_{n}(t)\right]^{T}=\Theta_{x}^{T}(t) \psi_{x}(t)
$$

where $\Theta_{x}^{T}(t)=\operatorname{diag}\left\{\theta_{x 1}^{T}(t), \theta_{x 2}^{T}(t), \ldots, \theta_{x n}^{T}(t)\right\}$.

Controller structure. Since the matrices $(A, B, C)$ of the plant (6.1.1) are unknown and the state sensors are with uncertainties, we choose $u(t)$ as

$$
u(t)=K_{1}^{T}(t) \hat{x}(t)+K_{2}(t) r(t)
$$

where $\hat{x}(t)$ is the state sensor uncertainty compensation signal, which is defined in (6.2.4), and $K_{1}(t) \in R^{n \times M}, K_{2}(t) \in R^{M \times M}$ are adaptive estimates of the unknown constant parameters $K_{1}^{*} \in R^{n \times M}$ and $K_{2}^{*} \in R^{M \times M}$, which satisfy the plant-model matching equation $(2.4 .3)$

$$
C\left(s I-A-B K_{1}^{* T}\right)^{-1} B K_{2}^{*}=W_{m}(s), K_{2}^{*-1}=K_{p}
$$

Remark 6.2.1. The parametrization of the controller signal $u(t)$ in (6.2.5) is not suitable for adaptive design, because both the parameter $\Theta_{x}^{T}(t)$ in the compensator $\hat{x}(t)=\Theta_{x}^{T}(t) \psi_{x}(t)$ and the parameter $K_{1}(t)$ in (6.2.5) need to be adaptively updated. We need to combine these two parameters together and derive a newly parameterized controller signal $u(t)$ to obtain an error equation suitable for adaptive law design.

New controller parametrization. From the state sensor compensator signal (6.2.4), we can have the new parameterized controller structure as

$$
u(t)=K_{x}^{T}(t) \psi_{x}(t)+K_{2}(t) r(t)
$$

where $K_{x}^{T}(t)=K_{1}^{T}(t) \Theta_{x}^{T}(t)$ is the estimate of $K_{x}^{* T}=K_{1}^{* T} \Theta_{x}^{* T}$, directly updated from an adaptive law. 
Remark 6.2.2. Although $\Theta_{x}^{T}(t)$ is a sparse matrix, we need to update all the components of the matrix $K_{x}^{T}(t)$, since the matrix $K_{1}^{T}(t) \Theta_{x}^{T}(t)$ is not sparse.

Substituting the newly parameterized controller signal (6.2.7) in the plant (6.1.1), and from (6.2.2), we have

$$
\begin{aligned}
\dot{x}(t) & =\left(A+B K_{1}^{* T}\right) x(t)+B K_{2}^{*} r(t)+B \widetilde{\Theta}^{T}(t) \omega(t) \\
y(t) & =C x(t),
\end{aligned}
$$

where $\widetilde{\Theta}(t)=\Theta(t)-\Theta^{*}, \Theta(t)=\left[K_{x}^{T}(t), K_{2}(t)\right]^{T}, \Theta^{*}=\left[K_{x}^{* T}, K_{2}^{*}\right]^{T}$, and $\omega(t)=$ $\left[\psi_{x}^{T}(t), r^{T}(t)\right]^{T}$

Since the output sensors have uncertainties, we can not obtain the exact output signal $y(t)$. But the adaptive laws would need the output tracking error information. Hence, we need to use the output sensor compensator to generate $\hat{y}(t)$, which is similar to the state sensor compensation signal $\hat{x}(t)$, to estimate the output signal $y(t)$ and obtain the compensation output tracking error $\hat{e}(t)=\hat{y}(t)-y_{m}(t)$ instead of the exact tracking error $e(t)=y(t)-y_{m}(t)$ for adaptive law design. Moreover, since $(A, B, C)$ is unknown, a new parametrization scheme is needed.

Output sensor compensator design. The model of the output sensors with uncertainties can be expressed as

$$
z_{y i}(t)=k_{y i} y_{i}(t)+\sum_{j=1}^{p_{i}} b_{y i j} f_{y i j}(t), i=1, \ldots, M
$$

where $k_{y i}, b_{y i j}, i=1, \ldots, M, j=1, \ldots, p_{i}$ are unknown constants, and $f_{y i j}(t), i=$ $1, \ldots, M, j=1, \ldots, p_{i}$ are known bounded signals. Then from (6.2.9), we can retrieve the output signals, which are given as

$$
y_{i}(t)=\theta_{y i}^{* T} \psi_{y i}(t)
$$


where $\theta_{y i}^{*}=\left[\theta_{\text {kyi }}^{*}, \theta_{\text {byi1 }}^{*}, \ldots, \theta_{\text {byip }}^{*}\right]^{T}$ and $\psi_{y i}(t)=\left[z_{y i}(t), f_{y i 1}(t), \ldots, f_{y i p_{i}}(t)\right]^{T}$ with $\theta_{\text {kyi }}^{*}=$ $1 / k_{y i}, \theta_{b y i j}^{*}=-b_{y i j} / k_{y i}$ being unknown constant parameters for $i=1,2, \ldots, M, j=$ $1,2, \ldots, p_{i}$. Then, the vector $y(t)$ is

$$
y(t)=\left[y_{1}(t), \ldots, y_{M}(t)\right]^{T}=\Theta_{y}^{* T} \psi_{y}(t)
$$

where $\Theta_{y}^{* T}=\operatorname{diag}\left\{\theta_{y 1}^{* T}, \theta_{y 2}^{* T}, \ldots, \theta_{y M}^{* T}\right\}$ and $\psi_{y}(t)=\left[\psi_{y 1}^{T}(t), \ldots, \psi_{y M}^{T}(t)\right]^{T}$. The output compensation signals $\hat{y}_{i}(t), i=1,2, \ldots, M$, are

$$
\hat{y}_{i}(t)=\theta_{y i}^{T}(t) \psi_{y i}(t)
$$

where $\theta_{y i}(t)$ is the estimate of $\theta_{y i}^{*}$. Then the output compensation signal $y(t)$ is

$$
\hat{y}(t)=\left[\hat{y}_{1}(t), \ldots, \hat{y}_{M}(t)\right]^{T}=\Theta_{y}^{T}(t) \psi_{y}(t)
$$

where $\Theta_{y}^{T}(t)=\operatorname{diag}\left\{\theta_{y 1}^{T}(t), \theta_{y 2}^{T}(t), \ldots, \theta_{y M}^{T}(t)\right\}$.

Parameter projection. The parameter vectors $\theta_{y i}(t)=\left[\theta_{k y i}, \theta_{\text {byi1 }}, \ldots, \theta_{\text {byip }}\right]^{T}$, $i=1,2, \ldots, M$, are to be updated from some adaptive laws. It is important to ensure that the parameters $\theta_{k y i}(t)$ do not get close to 0 , in order to guarantee closed-loop signal boundedness. This will be ensured by using parameter projection based algorithms [84], using the knowledge of $\theta_{k y i}^{0}>0$ such that $\theta_{k y i}^{0} \leq \theta_{k y i}^{*}$, for $i=1,2, \ldots, M$, to modify the adaptive laws so that $\theta_{k y i}^{0} \leq \theta_{k y i}(t)$, for $i=1,2, \ldots, M$, is ensured.

A parameter projection modification for an adaptive law: $\dot{\theta}_{k y i}(t)=g_{i}(t)$, is to add a signal $f_{g i}(t)$ (such that $f_{g i}(t)=0$ if $\theta_{k y i}^{0}<\theta_{k y i}(t)$ or if $\theta_{k y i}^{0}=\theta_{k y i}(t)$ and $g_{i}(t) \geq 0$, and $f_{g i}(t)=-g_{i}(t)$ otherwise, that is, if $\theta_{k y i}^{0}=\theta_{k y i}(t)$ and $\left.g_{i}(t)<0\right)$ to the adaptive law to form the new one: $\dot{\theta}_{k y i}(t)=g_{i}(t)+f_{g i}(t)$, with the condition on the initial estimate $\theta_{k y i}(0): \theta_{k y i}^{0} \leq \theta_{k y i}(0)$. Such a modified adaptive law has the desired properties: (i) $\theta_{k y i}^{0} \leq \theta_{k y i}(t)$ for all $t \geq 0$, and (ii) $\left(\theta_{k y i}(t)-\theta_{k y i}^{*}\right) f_{g i}(t) \leq 0$.

While it is not necessary (but may help) to use parameter projection for other components of $\theta_{y i}(t)=\left[\theta_{\text {kyi }}(t), \theta_{\text {byi1 }}(t), \ldots, \theta_{\text {byip }}(t)\right]^{T}, i=1,2, \ldots, M$, the adaptation 
gain matrix in the adaptive law for a parameter vector $\theta_{y i}(t)$ should be chosen to be a diagonal one [84].

Compensation tracking error. We introduce the compensation output tracking error signal

$$
\hat{e}(t)=\hat{y}(t)-y_{m}(t)=e(t)+(\hat{y}(t)-y(t))
$$

where $e(t)=y(t)-y_{m}(t)$ is the actual output tracking error.

In view of the reference model (6.1.5), the matching equations (6.2.6), the plant (6.2.8), the output signal (6.2.11), and the compensation output (6.2.13), we have

$$
\xi_{m}(s)[\hat{e}](t)=K_{p} \widetilde{\Theta}^{T}(t) \omega(t)+\xi_{m}(s)\left[\widetilde{\Theta}_{y}^{T} \psi_{y}\right](t),
$$

where $\widetilde{\Theta}_{y}(t)=\Theta_{y}(t)-\Theta_{y}^{*}$.

We introduce a filter $h(s)=\frac{1}{f(s)}$ with $f(s)$ being a stable and monic polynomial of degree equal to that of $\xi_{m}(s)$. Operating $h(s) I_{M}$ on both sides of $(6.2 .15)$, we have

$$
\tilde{e}(t)=K_{p} h(s)\left[\widetilde{\Theta}^{T} \omega\right](t)+\xi_{m}(s) h(s)\left[\widetilde{\Theta}_{y}^{T} \psi_{y}\right](t),
$$

where $\tilde{e}(t)=\xi_{m}(s) h(s)[\hat{e}](t)$.

Remark 6.2.3. This error equation has the output sensor uncertainty related term $\xi_{m}(s) h(s)\left[\widetilde{\Theta}_{y}^{T} \psi_{y}\right](t)$ which needs specific treatments, especially, in the presence of an uncertain gain matrix $K_{p}$ which introduces additional technical difficulties. In the absence of $\xi_{m}(s) h(s)\left[\widetilde{\Theta}_{y}^{T} \psi_{y}\right](t)$, the knowledge of $K_{p}$ can be relaxed, using $L D U, L D S$ and SDU decomposition based designs, for multivariable MRAC [84]. Such designs for sensor uncertainty compensation are still not yet available. For example, it was found that an LDS based design, directly applied to the error equation (6.2.16), may lead to an overparametrization problem (in deriving a linear error model) undesirable for system tracking. An SDU based design may be able to overcome such a problem and is currently under investigation. 
Estimation error. Based on (6.2.16), we introduce the estimation error signal

$$
\tilde{\epsilon}(t)=\tilde{e}(t)+\Psi(t) \xi(t)+\left[\xi_{y 11}(t), \sum_{i=1}^{2} \xi_{y 2 i}(t), \ldots, \sum_{i=1}^{M} \xi_{y M i}(t)\right]^{T}
$$

where $\Psi(t)$ is the estimate of $\Psi^{*}=K_{p}$, and

$$
\begin{aligned}
\xi(t) & =\Theta^{T}(t) \zeta(t)-h(s)\left[\Theta^{T} \omega\right](t), \\
\zeta(t) & =h(s)[\omega](t), \\
\xi_{y i j}(t) & =\theta_{y j}^{T}(t) \zeta_{y i j}(t)-h_{\xi i j}(s)\left[\theta_{y j}^{T} \psi_{y j}\right](t), \\
\zeta_{y i j}(t) & =h_{\xi i j}(s)\left[\psi_{y j}\right](t),
\end{aligned}
$$

with $h_{\xi i j}(s)$ being the $i j$ th component of $\xi_{m}(s) h(s)$, for $i=1, \ldots, M, j=1, \ldots, i$.

Substituting the filtered error $\tilde{e}(t)(6.2 .16)$ in (6.2.17), we have

$$
\tilde{\epsilon}(t)=\widetilde{\Psi}(t) \xi(t)+K_{p} \widetilde{\Theta}^{T}(t) \zeta(t)+\left[\begin{array}{c}
\tilde{\theta}_{y 1}^{T}(t) \zeta_{y 11}(t) \\
\sum_{i=1}^{2} \tilde{\theta}_{y i}^{T}(t) \zeta_{y 2 i}(t) \\
\vdots \\
\sum_{i=1}^{M} \tilde{\theta}_{y i}^{T}(t) \zeta_{y M i}(t)
\end{array}\right],
$$

where $\widetilde{\Psi}(t)=\Psi(t)-\Psi^{*}, \widetilde{\Theta}(t)$, and $\tilde{\theta}_{y i}(t)=\theta_{y i}(t)-\theta_{y i}^{*}, i=1,2, \ldots, M$, are the corresponding parameter errors.

Adaptive laws. With the estimation error (6.2.22), we choose the adaptive laws

$$
\begin{aligned}
\dot{\Psi}(t) & =-\frac{\Gamma \tilde{\epsilon}(t) \xi^{T}(t)}{m^{2}(t)}, \\
\dot{\Theta}^{T}(t) & =-\frac{S_{p} \tilde{\epsilon}(t) \zeta^{T}(t)}{m^{2}(t)}, \\
\dot{\theta}_{y i}(t) & =-\frac{\Gamma_{y i} \sum_{j=i}^{M} \tilde{\epsilon}_{j}(t) \zeta_{y j i}(t)}{m^{2}(t)}, i=1,2, \ldots, M,
\end{aligned}
$$

where $\tilde{\epsilon}(t)=\left[\tilde{\epsilon}_{1}(t), \tilde{\epsilon}_{2}(t), \ldots, \tilde{\epsilon}_{M}(t)\right]^{T}$ is computed from (6.2.17), in which $\tilde{e}(t)$ is computed from $\tilde{e}(t)=\xi_{m}(s) h(s)\left[\hat{y}-y_{m}\right](t)=\xi_{m}(s) h(s)\left[\Theta_{y}^{T} \psi_{y}-y_{m}\right](t), \Gamma=\Gamma^{T}>0$, and $\Gamma_{y i}=\Gamma_{y i}^{T}>0, i=1,2, \ldots, M$, are adaptation gain matrices, $S_{p}$ satisfies the 
Assumption (A3), and

$$
m^{2}(t)=1+\xi^{T}(t) \xi(t)+\zeta^{T}(t) \zeta(t)+\sum_{i=1}^{M} \sum_{j=1}^{i} \zeta_{y i j}^{T}(t) \zeta_{y i j}(t)
$$

Lemma 6.2.1. The adaptive laws (6.2.23)-(6.2.25) ensure that

(i) $\Psi(t) \in L^{\infty}, \Theta(t) \in L^{\infty}, \theta_{y i}(t) \in L^{\infty}, i=1,2, \ldots, M$, and $\frac{\tilde{\epsilon}(t)}{m(t)} \in L^{2} \cap L^{\infty}$; and

(ii) $\dot{\Psi}(t) \in L^{2} \cap L^{\infty}, \dot{\Theta}(t) \in L^{2} \cap L^{\infty}$, and $\dot{\theta}_{y i}(t) \in L^{2} \cap L^{\infty}, i=1,2, \ldots, M$.

Proof: Consider the positive definite function

$$
V=\frac{1}{2} \operatorname{tr}\left[\widetilde{\Psi}^{T} \Gamma^{-1} \widetilde{\Psi}\right]+\frac{1}{2} \operatorname{tr}\left[\widetilde{\Theta} \Gamma_{p} \widetilde{\Theta}^{T}\right]+\frac{1}{2} \sum_{i=1}^{M} \tilde{\theta}_{y i}^{T} \Gamma_{y i}^{-1} \tilde{\theta}_{y i} .
$$

From (6.2.23)-(6.2.25) and (6.2.22), we derive the derivative of $V$ as

$$
\begin{aligned}
\dot{V}= & -\frac{\xi^{T}(t) \widetilde{\Psi}^{T}(t) \tilde{\epsilon}(t)}{m^{2}(t)}-\frac{\zeta^{T}(t) \widetilde{\Theta}(t) K_{p}^{T} \tilde{\epsilon}(t)}{m^{2}(t)} \\
& -\sum_{i=1}^{M} \sum_{j=i}^{M} \frac{\tilde{\theta}_{y i}^{T}(t) \zeta_{y j i}(t) \tilde{\epsilon}_{j}(t)}{m^{2}(t)}=-\frac{\tilde{\epsilon}^{T}(t) \tilde{\epsilon}(t)}{m^{2}(t)} \leq 0 .
\end{aligned}
$$

From (6.2.28), we can conclude the properties in Lemma 1.

Based on Lemma 6.2.1, we can have the following theorem.

Theorem 6.2.1. The sensor uncertainty compensation scheme with the control law (6.2.7) updated by the adaptive laws (6.2.23)-(6.2.25), when applied to the plant (6.1.1), guarantees the closed-loop signal boundedness and asymptotic compensation output tracking: $\lim _{t \rightarrow \infty}\left(\hat{y}(t)-y_{m}(t)\right)=0$.

The convergence of the actual tracking error $e(t)=y(t)-y_{m}(t)$ to zero is still under investigation and it may need some additional conditions in the adaptive control system, as similar to an adaptive observer case where the adaptive state estimation error converges to zero under some persistent excitation conditions in the case when the system matrix $(A, B)$ are unknown. 


\subsection{Simulation Study}

In simulation study, we will consider an aircraft flight control example with system dynamics unknown and the output signals are a part of the state signals.

Aircraft model. We choose a linearized aircraft lateral model obtained by linearizing the NASA Generic Transport Model (GTM) at 70 knots trim condition, which is described as $\dot{x}(t)=A x(t)+B u(t), y(t)=C x(t)$, where $x=\left[v_{b}, p_{b}, r_{b}, \phi\right]^{T}$, $u=\left[d_{a}, d_{r}\right]^{T}$,

$$
\begin{aligned}
A= & {\left[\begin{array}{rrrr}
-0.5395 & 11.5510 & -117.4354 & 32.0436 \\
-0.5955 & -4.8871 & 2.0548 & 0 \\
0.2076 & -0.2891 & -1.3130 & 0 \\
0 & 1 & 0.0903 & \\
0
\end{array}\right] } \\
B= & {\left[\begin{array}{rr}
-0.0431 & 0.3774 \\
-0.5705 & 0.2181 \\
-0.0045 & -0.3569 \\
0 & 0
\end{array}\right], C=\left[\begin{array}{llll}
1 & 0 & 0 & 0 \\
0 & 0 & 0 & 1
\end{array}\right] . }
\end{aligned}
$$

The four state variables are the lateral velocity $v_{b}(\mathrm{ft} / \mathrm{sec})$, the roll rate $p_{b}(\mathrm{rad} / \mathrm{sec})$, the yaw rate $r_{b}(\mathrm{rad} / \mathrm{sec})$, and the roll angle $\phi(\mathrm{rad})$. The output variables are the lateral velocity $v_{b}$ and the roll angle $\phi$, which are two of the state signals. The control inputs are the aileron position $d_{a}(\mathrm{deg})$ and the rudder position $d_{r}(\mathrm{deg})$.

Sensor uncertainty models. We consider the state sensor uncertainties as

$$
\begin{aligned}
& z_{x 1}(t)=0.9 x_{1}(t)+0.2, z_{x 2}(t)=0.9 x_{2}(t), z_{x 3}(t)=x_{3}(t), \\
& z_{x 4}(t)=0.9 x_{4}(t)+0.01 \sin (0.03 t) .
\end{aligned}
$$

Since the output signals $y_{1}(t)$ and $y_{2}(t)$ are the state signals $x_{1}(t)$ and $x_{4}(t)$, the output sensor models are

$$
\begin{aligned}
& z_{y 1}(t)=z_{x 1}(t)=0.9 y_{1}(t)+0.2 \\
& z_{y 2}(t)=z_{x 4}(t)=0.9 y_{2}(t)+0.01 \sin (0.03 t)
\end{aligned}
$$


Reference model. The modified left interactor matrix $\xi_{m}(s)$ of $G(s)=C(s I-$ $A)^{-1} B$ can be chosen as $\xi_{m}(s)=\operatorname{diag}\left\{(s+1),(s+1)^{2}\right\}$. Therefore, the transfer matrix of the reference model $(6.1 .5)$ is $W_{m}(s)=\operatorname{diag}\left\{1 /(s+1), 1 /(s+1)^{2}\right\}$.

Verification of design conditions. The zero of $G(s)=C(s I-A)^{-1} B$ is $z=$ -118.33 which is stable, and $G(s)$ is strictly proper and has full rank. The high frequency gain matrix of $G(s)$ is

$$
K_{p}=\lim _{s \rightarrow \infty} \xi_{m}(s) G(s)=\left[\begin{array}{ll}
-0.0431 & 0.3774 \\
-0.5709 & 0.1859
\end{array}\right]
$$

which has full rank.

Design parameters. Since the degree of $\xi_{m}(s)$ is 2 , we choose the filter $h(s)=$ $1 /(s+1)^{2}$. For the adaptive laws $(6.2 .23)-(6.2 .25)$, we choose $\Gamma=\Gamma_{y 1}=\Gamma_{y 2}=$ $\operatorname{diag}\{1,1\}$, and

$$
S_{p}=\left[\begin{array}{cc}
-0.2155 & -2.8545 \\
3.7740 & 1.8590
\end{array}\right]
$$

which satisfies the Assumption (A3).

To prevent $\theta_{k y 1}(t)$ and $\theta_{k y 2}(t)$, which are updated in (6.2.25), getting close to 0 , we need to use parameter projection described in the "parameter projection" part after equation (6.2.13). From the output uncertainty models (6.3.3), we have $\theta_{k y 1}^{*}=$ $\theta_{k y 2}^{*}=1 / 0.9$. Then, we choose $\theta_{k y 1}^{0}=1<\theta_{k y 1}^{*}$ and $\theta_{k y 2}^{0}=1<\theta_{k y 2}^{*}$ as the lower bound of $\theta_{k y 1}(t)$ and $\theta_{k y 2}(t)$. For simulation, we choose the initial conditions as $\theta_{k y 1}(0)=1.2>\theta_{k y 1}^{0}, \quad \theta_{k y 2}(0)=1.2>\theta_{k y 2}^{0}$.

Simulation results. To make a realistic aircraft flying path, we choose the reference input $r(t)$ as in Case I the constant input $r(t)=[5,0.1]^{T}$, and Case II the varying reference input $r(t)=[5 \sin (0.01 t), 0.1 \sin (0.01 t)]^{T}$. By applying the compensation scheme using the adaptive laws (6.2.23)-(6.2.25), we can obtain the following simulation results. 
In Figure 6.2 and Figure 6.4, the dotted lines represent the reference output signal $y_{m}(t)$ and the solid lines represent the actual output signal $y(t)$ which are not available exactly because of the sensor uncertainties. From Figure 6.2 and Figure 6.4, we can see that, in Case I and Case II, the output signals are bounded and the tracking error $e(t)=y(t)-y_{m}(t)$ converges to small values.

Figure 6.3 and Figure 6.5 show that the compensator output signals $\hat{y}(t)$ (solid) asymptotically converge to the reference output signals $y_{m}(t)$ (dotted) for both Case I and Case II.
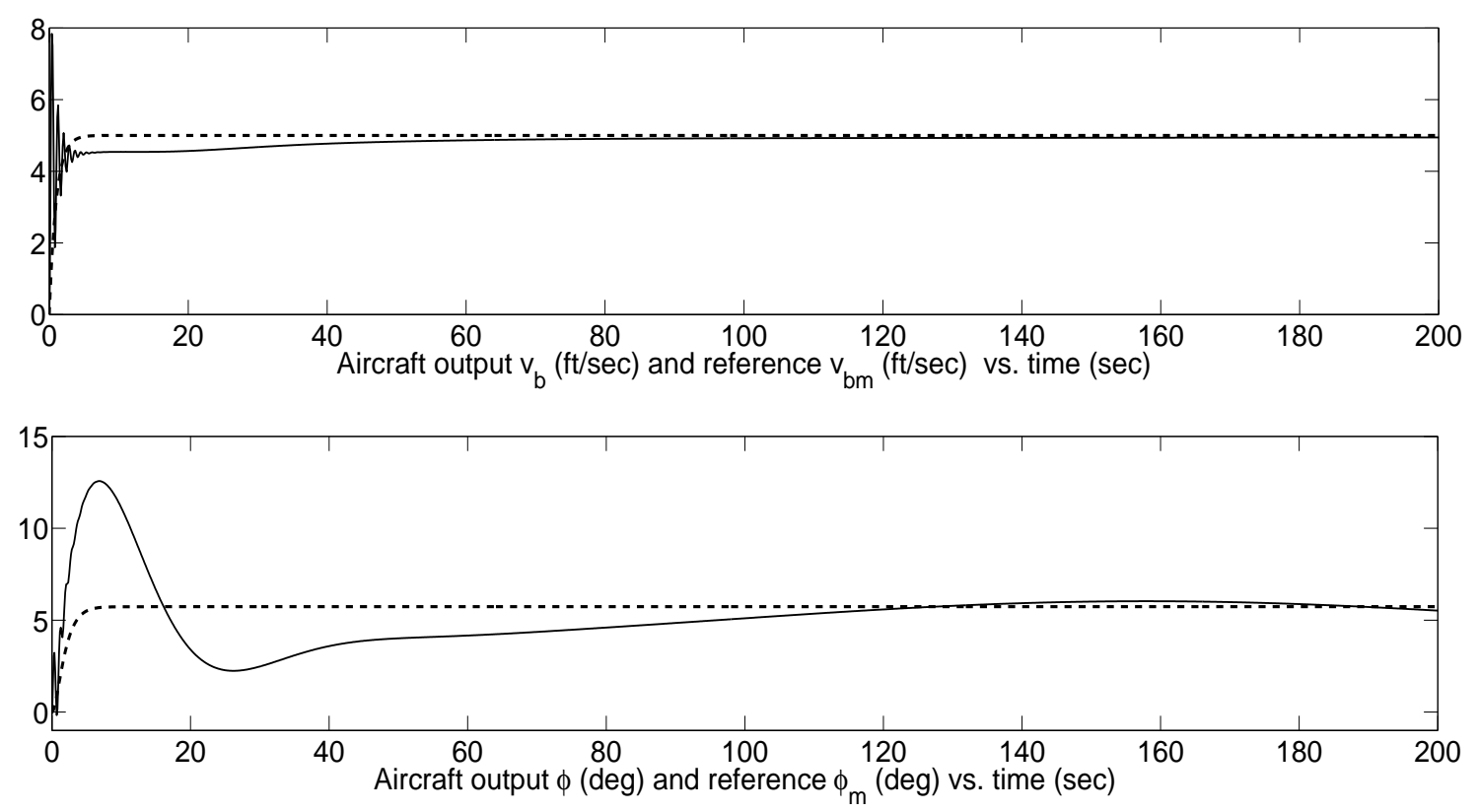

Figure 6.2: Aircraft output $y(t)$ (solid) vs. reference $y_{m}(t)$ (dotted) (Case I).

\section{Summary}

In this chapter, we have developed an adaptive sensor uncertainty compensation control scheme for the multi-input and multi-output systems with system dynamics unknown, using state feedback for output tracking. The critical step for an adap- 

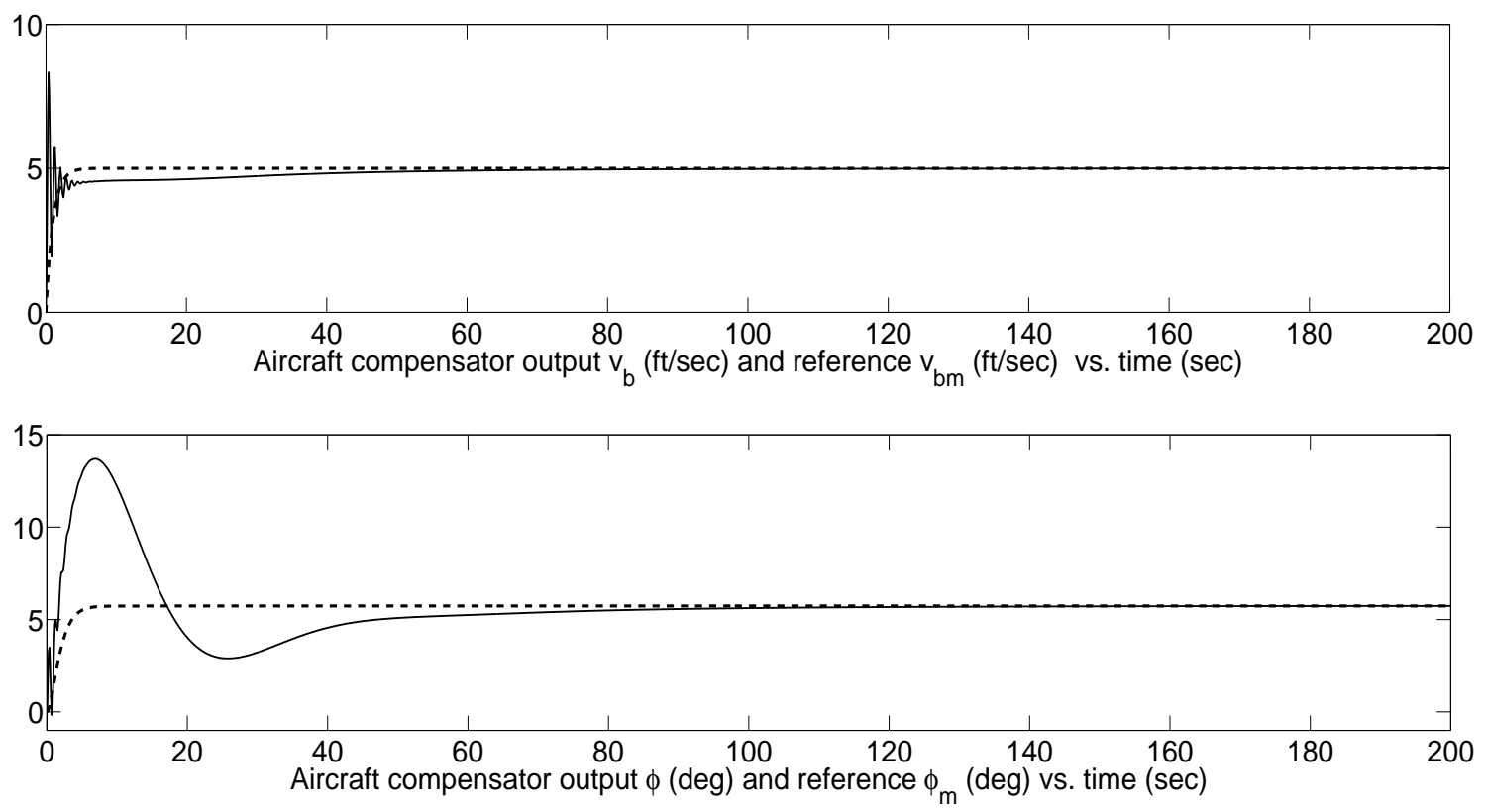

Figure 6.3: Compensator output $\hat{y}(t)$ (solid) vs. $y_{m}(t)$ (dotted) (Case I).
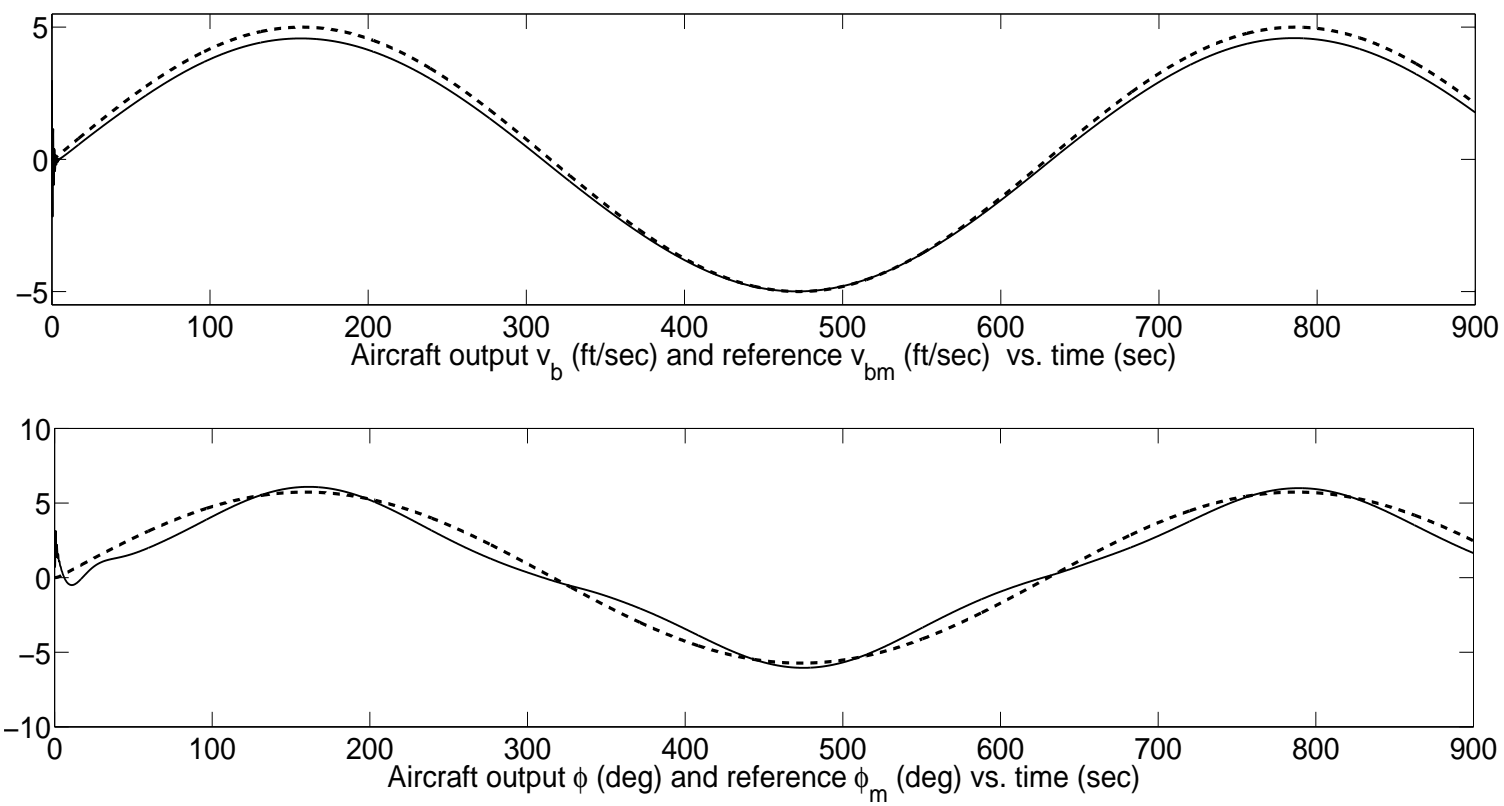

Figure 6.4: Aircraft output $y(t)$ (solid) vs. reference $y_{m}(t)$ (dotted) (Case II). 

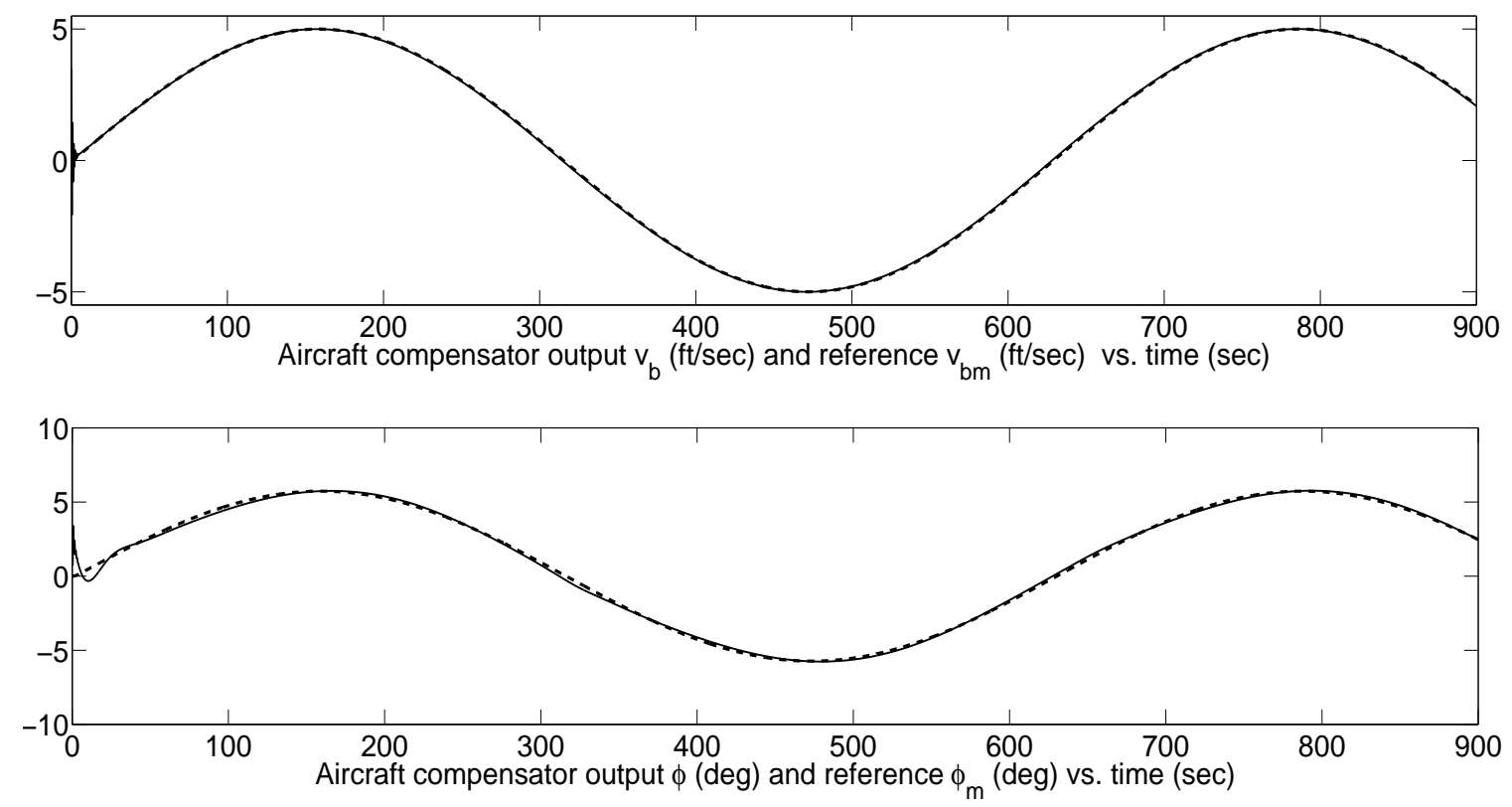

Figure 6.5: Compensator output $\hat{y}(t)$ (solid) vs. $y_{m}(t)$ (dotted) (Case II).

tive compensation design is the development of a properly parameterized error model in terms of the system and sensor parameter errors and the output tracking errors. We have developed such error model. Based on a suitable error model, stable adaptive laws have been derived for updating the parameters of the feedback controller. Simulation results have verified the desired performance. 


\section{Chapter 7}

\section{Discrete-Time Multivariable Adaptive Control of MIMO Systems with Structural Damage}

In this chapter, a discrete-time multivariable MRAC framework is developed for control of continuous-time nonlinear systems with structural damage, addressing several key technical issues. New technical contributions of this chapter in developing such digital control design techniques include

- obtaining a relationship of infinite zero structure between the continuous-time nonlinear system and its linearized discrete-time model;

- deriving invariance properties of infinite zero structure of the a linearized discretetime aircraft system model before and after damage occurs;

- developing a discrete-time multivariable MRAC scheme to compensate parametric and structural uncertainties due to damage and guarantee closed-loop system stability and asymptotic output tracking; and

- verifying effectiveness of the developed linearization-based discrete-time adaptive control framework for control of the continuous-time nonlinear system with damage by extensive simulation studies of the nonlinear GTM Simulink model. 


\subsection{Problem Statement}

Consider a continuous-time MIMO nonlinear system

$$
\dot{x}(t)=f(x(t))+g(x(t)) u(t), y(t)=C x(t),
$$

where $x(t) \in R^{n}$ and $u(t) \in R^{M}$ are state and control input vector signals, output vector signal $y(t) \in R^{M}$ is chosen as a linear combination of the state signals, and $f$ and $g_{i}, i=1,2, \ldots, M$, (with $g_{i}$ being the $i$ th column of $g$ ) are smooth (i.e., $C^{\infty}$ ) vector fields defined on $R^{n}$. When structural damage occurs, the system dynamics $f$ and $g$ in (7.1.1) may undergo uncertain parametric and structural variations. In this chapter, we will design a discrete-time adaptive control scheme to construct a digital control system framework with the addition of samplers and zero-order holds (ZOHs) for control of the nonlinear system (7.1.1) to compensate its possible structural damage.

For the digital control system with $\mathrm{ZOH}$, elements of the control input signal $u(t)=\left[u_{1}(t), u_{2}(t), \ldots, u_{M}(t)\right]^{T}$ of the nonlinear system (7.1.1) are

$$
u_{i}(t)=u_{i}(k T), \quad k T \leq t<(k+1) T
$$

for $i=1,2, \ldots, M$, i.e. within each sampling interval $T$, control input signals remain constant. We expand the state signal $x(t)$ in a Taylor series about $x(k T)$ within $t \in[k T,(k+1) T)$, it follows that $[5,50,60]$

$$
x((k+1) T)=x(k T)+\left.\sum_{i=1}^{\infty} \frac{T^{i}}{i !} \frac{\mathrm{d}^{i} x}{\mathrm{~d} t^{i}}\right|_{t=k T} .
$$

By taking successive partial derivatives of the right-hand side of (7.1.1) with control input signal as in (7.1.2), we can obtain a discrete-time nonlinear model denoted as

$$
x(k+1)=f_{d}(x(k), u(k)) .
$$

Since damage causes unknown variations for the nonlinear system (7.1.1), the discretized nonlinear model (7.1.4) also undergoes uncertain changes. To deal with the 
uncertainties and complexities of the discrete-time nonlinear model (7.1.4), in this chapter, we will employ a linearization-based discrete-time adaptive control design.

Linearization-based adaptive control. Block diagram of the closed-loop control system framework is illustrated in Fig. 7.1, where the linearization-based adaptive control design is applied. We linearize the discrete-time nonlinear model (7.1.4) at a chosen operating point $\left(x_{0}, u_{0}\right)$. The chosen operating point may not be an equilibrium point, since the equilibrium points may not be accessible due to system uncertainties. Then, a sequential discrete-time linear model with an unknown constant dynamics offset $f_{0}$ (introduced by the non-equilibrium operating point) can be used to characterize the linearized discrete-time system with damage:

$$
\Delta x(k+1)=A \Delta x(k)+B \Delta u(k)+f_{0}, \Delta y(k)=C \Delta x(k),
$$

where perturbation signals are $\Delta x(k)=x(k)-x_{0}, \Delta y(k)=y(k)-C x_{0}$, and $\Delta u(k)=$ $u(k)-u_{0}$, and system matrices and offset are $A=\left.\frac{\partial f_{d}}{\partial x}\right|_{\left(x_{0}, u_{0}\right)}, B=\left.\frac{\partial f_{d}}{\partial u}\right|_{\left(x_{0}, u_{0}\right)}$, and $f_{0}=f_{d}\left(x_{0}, u_{0}\right)-x_{0}$, which are unknown piecewise constants: $\left(A, B, f_{d 0}\right)=\left(A_{i}, B_{i}, f_{d 0_{i}}\right)$, $i=1, \ldots, N$, for $N$ different damage conditions.

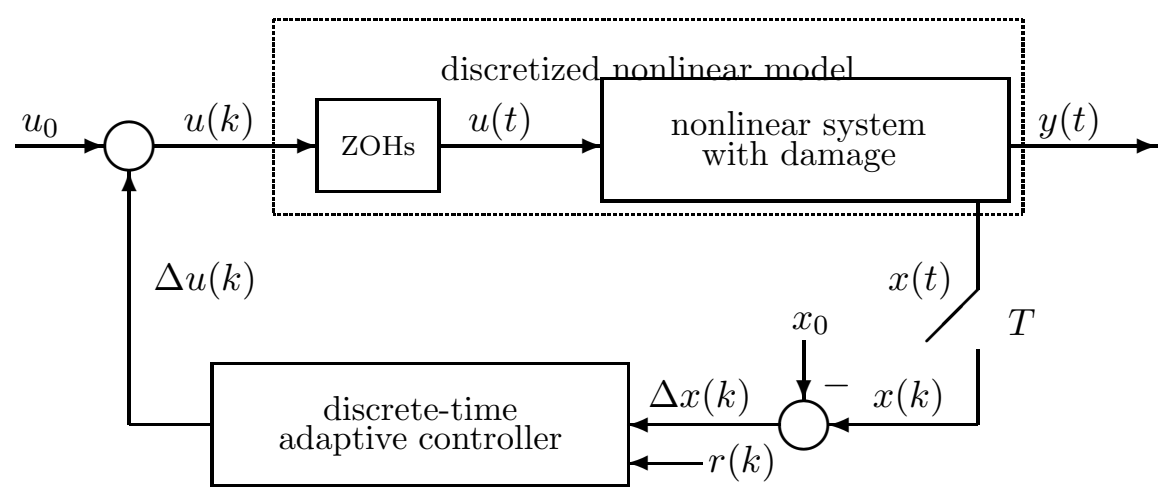

Figure 7.1: Digital control of a nonlinear system with damage.

Control objective. The objective is to develop a discrete-time adaptive control law $\Delta u(k)$ for the sequential linearized discrete-time system (7.1.5) with uncertainties 
to make all the signals of the closed-loop system bounded and the system output signal $\Delta y(k)$ asymptotically track a reference signal $\Delta y_{m}(k)$ :

$$
\Delta y_{m}(k)=W_{m}(z)[r](k), W_{m}(z)=\xi_{m}^{-1}(z)
$$

where $W_{m}(z)$ is stable and $r(k)$ is a bounded reference input signal. The symbol $z$ is used to denote the advance operator: $z[r](k)=r(k+1)$.

To proceed the multivariable MRAC design, for the sequential linearized discretetime system (7.1.5) with $\left(A, B, f_{d 0}\right)=\left(A_{i}, B_{i}, f_{d 0_{i}}\right), i=1,2, \ldots, N$, representing different damage conditions, following assumptions are required: (A1) all zeros of $G(z)=C(z I-A)^{-1} B$ lie within the unit circle in the z-plane; $(\mathrm{A} 2)(A, B)$ is controllable and $(A, C)$ is observable; (A3) $G(z)=C(z I-A)^{-1} B$ has full rank, for all damage scenarios, $G(z)$ has a common modified left interactor matrix $\xi_{m}(z)$; (A4) all leading principal minors $\Delta_{i}, i=1,2, \ldots, M$, of $K_{p}$ are nonzero, the sign of each principal minor is known and invariant for different damage scenarios.

(A1) is needed for stable zero-pole cancelations in plant-model matching (see (7.3.2)) (of making the closed-loop system be $\left.W_{m}(z)\right)$. (A2) is needed for ensuring system internal stability (based on observability of $(A, C)$, that is, a bounded output $y(k)$ implies a bounded state $x(k))$ and for plant-model matching (see (7.3.2)) (based on controllability of $(A, B))$. (A3) is for the existence of an interactor matrix $\xi_{m}(z)$ used for constructing a common reference model transfer matrix (7.1.6) for all possible damage scenarios. In next section, we will investigate the modified left interactor matrix $\xi_{m}(z)$ for the linearized discrete-time model (7.1.5) based on a detailed generic structure study. (A4) ensures that there exists a common matrix $D_{s}$ (a matrix of the LDS decomposition of $K_{p}$ defined in (7.3.12)) for all possible damage scenarios, which is used for design of the adaptive law (7.3.23). 


\subsection{System Infinite Zero Structures}

For discrete-time multivariable MRAC, knowledge of infinite zero structure is crucial for existence of plant-model matching (to be shown in Section 4), since the inverse of the interactor matrix $\xi_{m}(z)$ is chosen as the reference system (7.1.6). In this section, system infinite zero structures will be investigated to obtain a relationship between the continuous-time nonlinear system (7.1.1) and its linearized discrete-time model (7.1.5), based on which the invariance design conditions (A3) and (A4) may be obtained before and after damage (to be demonstrated by a generic study of an aircraft model in Section 7.4.1). In order to obtain a relationship of the infinite zero structures between the nonlinear system and its linearized discrete-time model, we first investigate the generic structure of the linearized discrete-time model (7.1.5).

\subsubsection{Linearized Discrete-Time System Model}

By taking successive partial derivatives of the right-hand side of (7.1.1) with the control input signals remaining constant in one sampling interval as in (7.1.2), the discretized nonlinear model (7.1.3) can be denoted as $[5,50,60]$

$$
\begin{aligned}
x(k+1)= & f_{d}(x(k), u(k)) \\
= & f_{d 0}(x(k))+\sum_{i=1}^{M} g_{d i}(x(k)) u_{i}(k) \\
& +\sum_{l=2}^{\infty}\left(\sum_{i_{1}=1}^{M} \ldots \sum_{i_{l}=1}^{M}\left(g_{d i_{1} \ldots i_{l}}(x(k)) \prod_{j=i_{1}}^{i_{l}} u_{j}(k)\right)\right) .
\end{aligned}
$$

Relative degrees of the discretized nonlinear model (7.2.1) can be defined [5,61,93], but, in this chapter, we only study the infinite zero structure of the linearized discretetime model for the discrete-time multivariable MRAC design.

We choose the operating point as $\left(x_{0}, u_{0}\right)=\left(x_{0}, 0\right)$ to linearize the discretized nonlinear system (7.2.1) to obtain the linearized discrete-time model (7.1.5). It follows 
that system matrices $A$ and $B$, and the offset $f_{0}$ of the linearized discrete-time model (7.1.5) are of the forms:

$$
\begin{aligned}
A & =\left.\frac{\partial f_{d}}{\partial x}\right|_{\left(x_{0}, 0\right)}=\left.\frac{\partial f_{d 0}}{\partial x}\right|_{x_{0}}, \\
B & =\left.\frac{\partial f_{d}}{\partial u}\right|_{\left(x_{0}, 0\right)}=\left[g_{d 1}\left(x_{0}\right), g_{d 2}\left(x_{0}\right), \ldots, g_{d M}\left(x_{0}\right)\right] \\
f_{0} & =f_{d}\left(x_{0}, 0\right)-x_{0}=f_{d 0}\left(x_{0}\right)-x_{0} .
\end{aligned}
$$

In order to obtain the expressions of $A, B$, and $f_{0}$ in terms of the continuous-time nonlinear system (7.1.1) vector fields $f$ and $g_{i}$, we further investigate $f_{d 0}$ and $g_{d i}$ in the discrete-time model (7.2.1), which can be expressed as

$$
\begin{aligned}
f_{d 0}(x(k))= & x(k)+\sum_{i=1}^{\infty} \frac{T^{i}}{i !} L_{f}^{i-1} f(x(k)) \\
g_{d i}(x(k))= & T g_{i}(x(k))+\sum_{l=2}^{\infty} \frac{T^{l}}{l !} L_{g_{i}} L_{f}^{l-2} f(x(k)) \\
& +\sum_{l=2}^{\infty} \frac{T^{l}}{l !} L_{f}^{l-1} g_{i}(x(k)) \\
& +\sum_{l=3}^{\infty} \frac{T^{l}}{l !}\left(\sum_{p=1}^{l-2} L_{f}^{p} L_{g_{i}} L_{f}^{l-p-2} f(x(k))\right),
\end{aligned}
$$

with $T$ denoting the sampling period, $g_{i}(x), i=1,2, \ldots, M$, being the $i$ th column of $g(x)$ of the nonlinear system (7.1.1), and the Lie derivatives defined as $L_{f}^{i+1} f=$ $L_{f}\left(L_{f}^{i} f\right)=\frac{\partial L_{f}^{i} f}{\partial x} f$ and $L_{f}^{0} f=f$. Substituting (7.2.5) and (7.2.6) in (7.2.2)-(7.2.4), we have

$$
\begin{aligned}
A & =\left.\frac{\partial f_{d 0}}{\partial x}\right|_{x_{0}}=I_{n}+\left.\sum_{i=1}^{\infty} \frac{T^{i}}{i !} \frac{\partial}{\partial x}\left(L_{f}^{i-1} f\right)\right|_{x_{0}}, \\
B & =\left[g_{d 1}\left(x_{0}\right), g_{d 2}\left(x_{0}\right), \ldots, g_{d M}\left(x_{0}\right)\right], \\
f_{0} & =f_{d 0}\left(x_{0}\right)-x_{0}=\sum_{i=1}^{\infty} \frac{T^{i}}{i !} L_{f}^{i-1} f\left(x_{0}\right) .
\end{aligned}
$$

Remark 7.2.1. If the operating point $u_{0}$ is non-zero, we can use a transformation $\bar{u}=u-u_{0}$ to transfer the nonlinear system (7.1.1) to an equivalent system $\dot{x}=$ 
$\bar{f}(x)+g(x) \bar{u}$, where $\bar{f}(x)=f(x)+g(x) u_{0}$, and then conduct analysis of the discretized nonlinear model and linearized discrete-time model for the system $\dot{x}=\bar{f}(x)+g(x) \bar{u}$ with the operating point $\left(x_{0}, 0\right)$.

When $\left(x_{0}, 0\right)$ is an equilibrium point of the nonlinear system (7.1.1), the system matrices and offset structures can be further simplified.

Proposition 7.2.1. If the operating point $\left(x_{0}, 0\right)$ is an equilibrium point of the continuoustime nonlinear system (7.1.1), then $\left(x_{0}, 0\right)$ is an equilibrium point of the discretized nonlinear system (7.2.1) as well, and the linearized discrete-time system matrices $A$ and $B$ as in (7.2.2) and (7.2.3), and the offset $f_{0}$ as in (7.2.4), are further reduced to

$$
A=e^{A_{c} T}, \quad B=\int_{0}^{T} e^{A_{c} \tau} B_{c} d \tau, \quad f_{0}=0,
$$

where $\left.A_{c} \triangleq \frac{\partial f}{\partial x}\right|_{x_{0}}$ and $B_{c} \triangleq g\left(x_{0}\right)$.

Proof: Substituting $\left(x_{0}, 0\right)$ in the right-hand side of the discretized nonlinear model (7.2.1) and in view of (7.2.5), we obtain that

$$
\begin{aligned}
f_{d}\left(x_{0}, 0\right) & =f_{d 0}\left(x_{0}\right)=x_{0}+\sum_{i=1}^{\infty} \frac{T^{i}}{i !} L_{f}^{i-1} f\left(x_{0}\right) \\
& =x_{0}+T f\left(x_{0}\right)+\left.\sum_{i=2}^{\infty} \frac{T^{i}}{i !} \frac{\partial}{\partial x}\left(L_{f}^{i-2} f\right)\right|_{x_{0}} f\left(x_{0}\right) .
\end{aligned}
$$

Since $\left(x_{0}, 0\right)$ is the equilibrium point of the continuous-time nonlinear system $\dot{x}=$ $f(x)+g(x) u$, we have that $f\left(x_{0}\right)=0$. From (7.2.11) and $f\left(x_{0}\right)=0$, it follows that

$$
f_{d}\left(x_{0}, 0\right)=x_{0}
$$

so that the operating point $\left(x_{0}, 0\right)$ is the equilibrium point for the discretized nonlinear model (7.2.1). Then the offset $f_{0}$ as in (7.2.4) becomes

$$
f_{0}=f_{d}\left(x_{0}, 0\right)-x_{0}=0
$$


With the condition $f\left(x_{0}\right)=0$, we have

$$
\begin{aligned}
\left.\frac{\partial}{\partial x}\left(L_{f}^{i-1} f\right)\right|_{x_{0}}= & \left.\frac{\partial}{\partial x}\left(\frac{\partial}{\partial x}\left(L_{f}^{i-2} f\right) \cdot f\right)\right|_{x_{0}} \\
= & \left.\left.\frac{\partial}{\partial x}\left(L_{f}^{i-2} f\right)\right|_{x_{0}} \frac{\partial f}{\partial x}\right|_{x_{0}} \\
& +\left[\left.f^{T}\left(x_{0}\right) \frac{\partial}{\partial x}\left(\left[\frac{\partial}{\partial x}\left(L_{f}^{i-2} f\right)\right]_{1}^{T}\right)\right|_{x_{0}}\right] \\
\vdots & \\
= & \left.\left.\frac{\partial}{\partial x}\left(f_{f}^{i-2} f\right)\right|_{x_{0}} \frac{\partial f}{\partial x}\right|_{x_{0}} \\
= & \cdots \\
= & \left(\left.\frac{\partial f}{\partial x}\right|_{x_{0}}\right)^{i}, i=2,3, \ldots
\end{aligned}
$$

where $\left[\frac{\partial}{\partial x}\left(L_{f}^{i-2} f\right)\right]_{j}, j=1,2, \ldots, n$, denotes the $j$ th row of the matrix $\frac{\partial}{\partial x}\left(L_{f}^{i-2} f\right)$. Hence, from (7.2.14), the matrix $A$ in (7.2.7) can be further calculated as

$$
\begin{aligned}
A & =I_{n}+\left.T \frac{\partial f}{\partial x}\right|_{x_{0}}+\left.\sum_{i=2}^{\infty} \frac{T^{i}}{i !} \frac{\partial}{\partial x}\left(L_{f}^{i-1} f\right)\right|_{x_{0}} \\
& =I_{n}+\sum_{i=1}^{\infty} \frac{T^{i}}{i !}\left(\left.\frac{\partial f}{\partial x}\right|_{x_{0}}\right)^{i} .
\end{aligned}
$$

In view of $(7.2 .6),(7.2 .3),(7.2 .14)$, and the condition $f\left(x_{0}\right)=0$, we have the $i$ th column of matrix $B$ as

$$
\begin{aligned}
b_{i}= & g_{d i}\left(x_{0}\right) \\
= & T g_{i}\left(x_{0}\right)+\left.\sum_{l=2}^{\infty} \frac{T^{l}}{l !} \frac{\partial}{\partial x}\left(L_{f}^{l-2} f\right)\right|_{x_{0}} g_{i}\left(x_{0}\right) \\
& +\left.\sum_{l=2}^{\infty} \frac{T^{l}}{l !} \frac{\partial}{\partial x}\left(L_{f}^{l-2} g_{i}\right)\right|_{x_{0}} f\left(x_{0}\right) \\
& +\sum_{l=3}^{\infty} \frac{T^{l}}{l !}\left(\left.\sum_{p=1}^{l-2} \frac{\partial}{\partial x}\left(L_{f}^{p-1} L_{g_{i}} L_{f}^{l-p-2} f\right)\right|_{x_{0}} f\left(x_{0}\right)\right) \\
= & T g_{i}\left(x_{0}\right)+\sum_{l=2}^{\infty} \frac{T^{l}}{l !}\left(\left.\frac{\partial f}{\partial x}\right|_{x_{0}}\right)^{l-1} g_{i}\left(x_{0}\right),
\end{aligned}
$$


with $b_{i}, i=1,2, \ldots, M$, denoting the $i$ th column of $B$. The matrices $A$ as in (7.2.15) and $B$ with the $i$ th column $b_{i}$ as in (7.2.16) can be expressed as more compact forms:

$$
A=e^{A_{c} T}, \quad B=\int_{0}^{T} e^{A_{c} \tau} B_{c} d \tau
$$

where we define $A_{c}=\left.\frac{\partial f}{\partial x}\right|_{x_{0}}$ and $B_{c}=g\left(x_{0}\right)=\left[g_{1}\left(x_{0}\right), g_{2}\left(x_{0}\right), \ldots, g_{M}\left(x_{0}\right)\right]$.

Remark 7.2.2. There are two methods to obtain a linear discrete-time model as in (7.1.5) from the continuous-time nonlinear system (7.1.1): one is discretizing the nonlinear system (7.1.1) first, and then linearizing the discretized model, which is used in this chapter; the other one is linearizing the nonlinear system (7.1.1) first, and then discretizing the linearized model. It is worth noting that $A$ and $B$ in (7.2.10) are actually system matrices of the discrete-time linear model obtained from the second method with the operating point as $\left(x_{0}, 0\right)$. That is only if $\left(x_{0}, 0\right)$ is an equilibrium point, system matrices $A$ and $B$ obtained from these two methods are the same. When $\left(x_{0}, 0\right)$ is an arbitrary operating point, $A$ and $B$ from the first method as in (7.2.7) and (7.2.8) contain more information than the second method as in (7.2.10). Therefore, in this chapter, we use the linearized discrete-time model from the first method to do the linearization-based design.

\subsubsection{Interactor Matrix of Linearized Discrete-Time Model}

We use the following calculation of $G(z)=C\left(z I_{n}-A\right)^{-1} B$ to determine the infinite zero structure of the linearized discrete-time system (7.1.5):

$$
G(z)=\frac{1}{\operatorname{det}\left(z I_{n}-A\right)}\left(E_{n-1} z^{n-1}+\cdots+E_{1} z+E_{0}\right)
$$

with $\operatorname{det}\left(z I_{n}-A\right) \triangleq z^{n}+\alpha_{n-1} z^{n-1}+\cdots+\alpha_{0}$ and coefficients being $E_{n-1}=C B, E_{n-2}=$ $C A B+\alpha_{n-1} C B, \ldots, E_{0}=C A^{n-1} B+\alpha_{n-1} C A^{n-2} B+\cdots+\alpha_{1} C B$. 
From expressions of matrices $A$ and $B$ in (7.2.7) and (7.2.8), which are related to the continuous-time nonlinear system (7.1.1) at the operating point $x_{0}$, we obtain following properties about the infinite zero structures.

Theorem 7.2.1. For the nonlinear system (7.1.1):

$$
\dot{x}=f(x)+\sum_{i=1}^{M} g_{i}(x) u_{i}, y_{i}=h_{i}(x)=C_{i} x, i=1, \ldots, M
$$

with the relative degree $\left\{\rho_{1}, \rho_{2}, \ldots, \rho_{M}\right\}$ at the point $x_{0}$, there exist small constants $T_{0}>0$ and $f_{n}>0$, such that, if $T<T_{0}$, i.e., the sampling period is sufficiently small, and $\left\|f\left(x_{0}\right)\right\|<f_{n}$, i.e., the operating point $\left(x_{0}, 0\right)$ is sufficiently close to the equilibrium point, the linearized discrete-time system (7.1.5) at the $\left(x_{0}, 0\right)$ :

$$
\Delta x(k+1)=A \Delta x(k)+B \Delta u(k)+f_{0}, \Delta y(k)=C \Delta x(k),
$$

with $A, B$, and $f_{0}$, as in (7.2.7)-(7.2.9), has a diagonal modified left interactor matrix:

$$
\xi_{m}(z)=\operatorname{diag}\left\{z+a_{1}, z+a_{2}, \ldots, z+a_{M}\right\}
$$

with $\left|a_{i}\right|<1$, for $i=1,2, \ldots, M$.

Proof: For the nonlinear system (7.2.19) with the relative degree $\left\{\rho_{1}, \rho_{2}, \ldots, \rho_{M}\right\}$ at the point $x_{0}$, we have $L_{g_{j}} L_{f}^{k} h_{i}(x)=0, j=1, \ldots, M, k=0, \ldots, \rho_{i}-2, i=1, \ldots, M$, in a neighborhood of $x_{0}$, and the matrix

$$
\alpha\left(x_{0}\right)=\left[\begin{array}{ccc}
L_{g_{1}} L_{f}^{\rho_{1}-1} h_{1}\left(x_{0}\right) & \cdots & L_{g_{M}} L_{f}^{\rho_{1}-1} h_{1}\left(x_{0}\right) \\
L_{g_{1}} L_{f}^{\rho_{2}-1} h_{2}\left(x_{0}\right) & \cdots & L_{g_{M}} L_{f}^{\rho_{2}-1} h_{2}\left(x_{0}\right) \\
\vdots & \vdots & \vdots \\
L_{g_{1}} L_{f}^{\rho_{M}-1} h_{M}\left(x_{0}\right) & \cdots & L_{g_{M}} L_{f}^{\rho_{M}-1} h_{M}\left(x_{0}\right)
\end{array}\right]
$$

is non-singular, with the Lie derivatives defined as $L_{f}^{k} h_{i}=L_{f}\left(L_{f}^{k-1} h_{i}\right)=\frac{\partial L_{f}^{k-1} h_{i}}{\partial x} f$, $L_{f}^{0} h_{i}=h_{i}$, and $L_{g_{j}} L_{f}^{k} h_{i}=\frac{\partial}{\partial x}\left(L_{f}^{k} h_{i}\right) g_{j}[43,84]$. For the Lie derivative $L_{g_{j}} L_{f}^{k} h_{i}(x)$, 
$k \geq 1$, we can express it as

$$
\begin{aligned}
L_{g_{j}} L_{f}^{k} h_{i}= & \frac{\partial}{\partial x}\left(L_{f}^{k} h_{i}\right) g_{j} \\
= & \frac{\partial h_{i}}{\partial x} \frac{\partial}{\partial x}\left(L_{f}^{k-1} f\right) g_{j}+\left(L_{f}^{k-1} f\right)^{T} \frac{\partial}{\partial x}\left(\left(\frac{\partial h_{i}}{\partial x}\right)^{T}\right) g_{j} \\
& +\sum_{l=0}^{k-2} \frac{\partial}{\partial x}\left(L_{f}^{l}\left(\left(L_{f}^{k-2-l} f\right)^{T} \cdot \frac{\partial}{\partial x}\left(\left(\frac{\partial h_{i}}{\partial x}\right)^{T}\right) \cdot f\right)\right) g_{j}
\end{aligned}
$$

Since the output is a linear combination of the states, i.e. $h_{i}(x)=C_{i} x$, we can simplify $L_{g_{j}} L_{f}^{k} h_{i}(x)$ as

$$
\begin{aligned}
L_{g_{j}} h_{i}(x) & =\frac{\partial h_{i}}{\partial x} g_{j}(x)=C_{i} g_{j}(x), \\
L_{g_{j}} L_{f}^{k} h_{i}(x) & =\frac{\partial h_{i}}{\partial x} \frac{\partial}{\partial x}\left(L_{f}^{k-1} f\right) g_{j}(x) \\
& =C_{i} \frac{\partial}{\partial x}\left(L_{f}^{k-1} f\right) g_{j}(x) \\
& =C_{i} L_{g_{j}} L_{f}^{k-1} f(x), \quad k \geq 1 .
\end{aligned}
$$

Then, we have that $\forall x$ in a neighborhood of $x_{0}$

$$
C_{i} g_{j}(x)=0, \quad C_{i} L_{g_{j}} L_{f}^{k-1} f(x)=0,1 \leq k<\rho_{i}-1
$$

and the non-singular $\alpha\left(x_{0}\right)$ is reduced to

$$
\alpha\left(x_{0}\right)=\left[\begin{array}{ccc}
C_{1} L_{g_{1}} L_{f}^{\rho_{1}-2} f & \cdots & C_{1} L_{g_{M}} L_{f}^{\rho_{1}-2} f \\
C_{2} L_{g_{1}} L_{f}^{\rho_{2}-2} f & \cdots & C_{2} L_{g_{M}} L_{f}^{\rho_{2}-2} f \\
\vdots & \vdots & \vdots \\
C_{M} L_{g_{1}} L_{f}^{\rho_{M}-2} f & \cdots & C_{M} L_{g_{M}} L_{f}^{\rho_{M}-2} f
\end{array}\right] .
$$

To derive the interactor matrix for $G(z)$, we investigate the first coefficient $E_{n-1}=C B$ of the equation (7.2.18). For the $i$ th row $E_{(n-1), i}=\left[C_{i} B_{1}, C_{i} B_{2}, \ldots, C_{i} B_{M}\right]$, from 
(7.2.3) and (7.2.26), we obtain each element $C_{i} B_{j}, j=1,2, \ldots, M$ as

$$
\begin{aligned}
C_{i} B_{j}= & \frac{T^{\rho_{i}}}{\rho_{i} !} C_{i} L_{g_{j}} L_{f}^{\rho_{i}-2} f\left(x_{0}\right)+\sum_{l=\rho_{i}+1}^{\infty} \frac{T^{l}}{l !} C_{i} L_{g_{i}} L_{f}^{l-2} f\left(x_{0}\right) \\
& +\sum_{l=3}^{\infty} \frac{T^{l}}{l !} C_{i}\left(L_{f}^{l-1} g_{i}\left(x_{0}\right)+\sum_{p=1}^{l-2} L_{f}^{p} L_{g_{i}} L_{f}^{l-p-2} f\left(x_{0}\right)\right) \\
= & \frac{T^{\rho_{i}}}{\rho_{i} !} C_{i} L_{g_{j}} L_{f}^{\rho_{i}-2} f\left(x_{0}\right)+\sum_{l=\rho_{i}+1}^{\infty} \frac{T^{l}}{l !} C_{i} L_{g_{i}} L_{f}^{l-2} f\left(x_{0}\right) \\
& +\sum_{l=3}^{\infty} \frac{T^{l}}{l !} C_{i}\left(\left.\frac{\partial}{\partial x}\left(L_{f}^{l-2} g_{i}\right)\right|_{x_{0}} f\left(x_{0}\right)\right) \\
& +\sum_{l=3}^{\infty} \frac{T^{l}}{l !} C_{i}\left(\sum_{p=1}^{l-2}\left(\left.\frac{\partial}{\partial x}\left(L_{f}^{p-1} L_{g_{i}} L_{f}^{l-p-2} f\right)\right|_{x_{0}} \cdot f\left(x_{0}\right)\right)\right) .
\end{aligned}
$$

From (7.2.28), there exist small $T_{0}>0$ and $f_{n}>0$, such that, if $T<T_{0}$ and $\left\|f\left(x_{0}\right)\right\|<f_{n}, C_{i} B_{j}$ can be approximated as

$$
C_{i} B_{j} \approx \frac{T^{\rho_{i}}}{\rho_{i} !} C_{i} L_{g_{j}} L_{f}^{\rho_{i}-2} f\left(x_{0}\right) .
$$

From (7.2.27) and (7.2.29), it follows that

$$
E_{n-1}=C B \approx P \alpha\left(x_{0}\right)
$$

where $P=\operatorname{diag}\left\{\frac{T^{\rho_{1}}}{\rho_{1} !}, \frac{T^{\rho_{2}}}{\rho_{2} !}, \ldots, \frac{T^{\rho_{M}}}{\rho_{M} !}\right\}$, which is non-singular. Since the matrix $\alpha\left(x_{0}\right)$ is non-singular, we can conclude that the coefficient $E_{n-1}=C B$ is non-singular, when the sampling period $T<T_{0}$ and $\left\|f\left(x_{0}\right)\right\|<f_{n}$, i.e., the operating point $\left(x_{0}, 0\right)$ is close to the equilibrium point. Hence, we choose the interactor matrix $\xi_{m}(z)$ as

$$
\xi_{m}(z)=\operatorname{diag}\left\{z+a_{1}, z+a_{2}, \ldots, z+a_{M}\right\}
$$

with $\left|a_{i}\right|<1, i=1, \ldots, M$ to make $\lim _{z \rightarrow \infty} \xi_{m}(z) G(z)=E_{n-1}$ non-singular.

From the proof of Theorem 7.2.1, we can see that if the relative degree $\rho_{i} \leq 2$, the condition on the operating point $\left(x_{0}, 0\right)$, i.e., $\left\|f\left(x_{0}\right)\right\|<f_{n}$, can be relaxed. This property is summarized as the following corollary. 
Corollary 7.2.1. For the continuous-time nonlinear system (7.2.19) with the relative degree $\left\{\rho_{1}, \rho_{2}, \ldots, \rho_{M}\right\}$ at the point $x_{0}$, where $\rho_{i} \leq 2, i=1,2, \ldots, M$, there exists a small $T_{0}>0$, such that, if the sampling period $T<T_{0}$, the linearized discrete-time system (7.2.20) at the operating point $\left(x_{0}, 0\right)$ with $A, B$, and $f_{0}$, given as (7.2.7)(7.2.9), has a diagonal left modified interactor matrix: $\xi_{m}(z)=\operatorname{diag}\left\{z+a_{1}, z+\right.$ $\left.a_{2}, \ldots, z+a_{M}\right\}$, with $\left|a_{i}\right|<1$, for $i=1,2, \ldots, M$.

Proof: For $\rho_{i}=1$, we have that the $i$ th row of $\alpha\left(x_{0}\right)$ is

$$
\alpha_{i}\left(x_{0}\right)=\left[L_{g_{1}} h_{i}\left(x_{0}\right), \ldots, L_{g_{M}} h_{i}\left(x_{0}\right)\right]=C_{i} g\left(x_{0}\right) \neq 0 .
$$

For $\rho_{i}=2$, we obtain that, $\forall x$ in a neighborhood of $x_{0}, L_{g_{j}} h_{i}(x)=C_{i} g_{j}(x)=0$, $j=1,2, \ldots, M$, and the $i$ th row of $\alpha\left(x_{0}\right)$ is

$$
\begin{aligned}
\alpha_{i}\left(x_{0}\right) & =\left[L_{g_{1}} L_{f} h_{i}\left(x_{0}\right), \ldots, L_{g_{M}} L_{f} h_{i}\left(x_{0}\right)\right] \\
& =\left[C_{i} L_{g_{1}} f\left(x_{0}\right), \ldots, C_{i} L_{g_{M}} f\left(x_{0}\right)\right] \neq 0 .
\end{aligned}
$$

From (7.2.3), we have that the $i$ th row of the coefficient $E_{n-1}=C B$ of (7.2.18) is

$$
\begin{aligned}
C_{i} B_{j}= & T C_{i} g_{j}\left(x_{0}\right)+\frac{T^{2}}{2} C_{i} L_{g_{j}} f\left(x_{0}\right)+\sum_{l=3}^{\infty} \frac{T^{l}}{l !} C_{i} L_{g_{i}} L_{f}^{l-2} f\left(x_{0}\right) \\
& +\sum_{l=3}^{\infty} \frac{T^{l}}{l !} C_{i}\left(L_{f}^{l-1} g_{i}\left(x_{0}\right)+\sum_{p=1}^{l-2} L_{f}^{p} L_{g_{i}} L_{f}^{l-p-2} f\left(x_{0}\right)\right) .
\end{aligned}
$$

There exists a small $T_{0}>0$, such that, for $T<T_{0}$, the coefficient is approximated as

$$
C_{i} B_{j} \approx T C_{i} g_{j}\left(x_{0}\right)+\frac{T^{2}}{2} C_{i} L_{g_{j}} f\left(x_{0}\right) .
$$

It follows that

$$
E_{n-1}=C B \approx P \alpha\left(x_{0}\right)
$$

where $P$ is a diagonal matrix with the diagonal elements are $T$ or $\frac{T^{2}}{2}$. Hence, the first coefficient $E_{n-1}$ is non-singular. So we choose the interactor matrix as

$$
\xi_{m}(z)=\operatorname{diag}\left\{z+a_{1}, z+a_{2}, \ldots, z+a_{M}\right\}
$$


with $\left|a_{i}\right|<1$, for $i=1,2, \ldots, M$, such that $K_{p}=\lim _{z \rightarrow \infty} \xi_{m}(z) G(z)=C B$, which is non-singular.

Theorem 7.2.1 and Corollary 7.2.1 indicate that, when structural damage occurs, even if the relative degree of the nonlinear system is altered, infinite zero structure of the linearized discrete-time model can be invariant, so that the key invariance conditions (A3) can hold for adaptive damage compensation design.

\subsection{A Multivariable MRAC Scheme}

In this section, we will develop a new multivariable state feedback MRAC scheme for the sequential linearized discrete-time model (7.1.5) with parametric uncertainties due to damage.

We consider a state feedback controller, which has a simple structure suitable for aircraft control and some other applications:

$$
\Delta u(k)=K_{1}^{T}(k) \Delta x(k)+K_{2}(k) r(k)+K_{3}(k),
$$

where $K_{3}(k)$ is the adaptive estimate of the unknown compensation term $K_{3}^{*}$ for canceling the effect of the piecewise constant offset $f_{0}$, and $K_{1}(k)$ and $K_{2}(k)$ are the estimates of the nominal $K_{1}^{*}$ and $K_{2}^{*}$, which satisfy the plant-model matching condition (2.4.3):

$$
C\left(z I-A-B K_{1}^{* T}\right)^{-1} B K_{2}^{*}=W_{m}(z), K_{2}^{*-1}=K_{p}
$$

where the reference system transfer matrix $W_{m}(z)$ is the inverse of the modified left interactor matrix: $W_{m}(z)=\xi_{m}^{-1}(z)$. In particular, when the interactor matrix $\xi_{m}(z)$ is of a diagonal form, the existence of $K_{1}^{*}$ and $K_{2}^{*}$ of the matching condition (7.3.2) can be treated as the solution of the dynamic decoupling problem for multivariable systems, which is stated and solved in [22]; when the interactor matrix $\xi_{m}(z)$ is of 
a lower triangular form, [62] gives a method to solve $K_{1}^{*}$ and $K_{2}^{*}$ for the matching condition (7.3.2). To derive $K_{3}^{*}$, we apply the nominal controller

$$
\Delta u(k)=K_{1}^{* T} \Delta x(k)+K_{2}^{*} r(k)+K_{3}^{*}
$$

to the system (7.1.5). Considering a set of $A, B$, and $f_{0}$, we have the closed-loop system in the $z$-domain as

$$
\Delta y(z)=C\left(z I-A-B K_{1}^{* T}\right)^{-1} B K_{2}^{*} r(z)+\Delta(z)
$$

with $\Delta(z)=C\left(z I-A-B K_{1}^{* T}\right)^{-1}\left(B \frac{K_{3}^{*} z}{z-1}+\frac{f_{0} z}{z-1}\right)$. In view of the reference system (7.1.6):

$$
\Delta y_{m}(k)=W_{m}(z)[r](k), W_{m}(z)=\xi_{m}^{-1}(z),
$$

and the matching condition (7.3.2), we have the output tracking error as

$$
\Delta e(z)=\Delta y(z)-\Delta y_{m}(z)=\Delta(z)
$$

Applying the $z$-domain final value theorem, we obtain

$$
\lim _{k \rightarrow \infty} \Delta e(k)=\lim _{z \rightarrow 1}(z-1) \Delta(z)=W_{m}(1) K_{p} K_{3}^{*}+d
$$

with $d=C\left(I-A-B K_{1}^{* T}\right)^{-1} f_{0}$. Then, we set

$$
K_{3}^{*}=-K_{p}^{-1} \xi_{m}(1) d
$$

which follows that $\lim _{k \rightarrow \infty}\left(\Delta y(k)-\Delta y_{m}(k)\right)=0$. Therefore, there exists a nominal controller (7.3.3) with $K_{1}^{*}, K_{2}^{*}$, and $K_{3}^{*}$, satisfying (7.3.2) and (7.3.8) to make the output signal $\Delta y(k)$ of the linearized discrete-time model (7.1.5) track a reference signal $\Delta y_{m}(k): \lim _{k \rightarrow \infty}\left(\Delta y(k)-\Delta y_{m}(k)\right)=0$.

Remark 7.3.1. The multivariable discrete-time MRAC control scheme developed in this chapter is applicable for linear discrete-time systems (7.1.5) with general interactor matrix $\xi_{m}(z)$. In this chapter, for the linearization-based discrete-time adaptive control design, the interactor matrix $\xi_{m}(z)$ of the linearized discrete-time system 
(7.1.5) possesses a diagonal form with relative degree 1 (as shown in (7.2.21)) and is invariant before and after damage occurs, so that there exists a common stable reference system $W_{m}(z)=\xi_{m}^{-1}(z)=\operatorname{diag}\left\{\left(z+a_{1}\right)^{-1},\left(z+a_{2}\right)^{-1}, \ldots,\left(z+a_{M}\right)^{-1}\right\}$ with $\left|a_{i}\right|<1, i=1,2, \ldots, M$, for all possible damage patterns $\left(A_{i}, B_{i}\right), i=1,2, \ldots, N$.

Tracking error equation. Substituting the adaptive control law (7.3.1) in the plant (7.1.5), we have

$$
\begin{aligned}
\Delta x(k+1)= & \left(A+B K_{1}^{* T}\right) \Delta x(k)+B K_{2}^{*} r(k)+B K_{3}^{*}+f_{0} \\
& +B\left(\widetilde{K}_{1}^{T}(k) \Delta x(k)+\widetilde{K}_{2}(k) r(k)+\widetilde{K}_{3}(k)\right) \\
\Delta y(k)= & C \Delta x(k),
\end{aligned}
$$

where $\widetilde{K}_{1}(k)=K_{1}(k)-K_{1}^{*}, \widetilde{K}_{2}(k)=K_{2}(k)-K_{2}^{*}$, and $\widetilde{K}_{3}(k)=K_{3}(k)-K_{3}^{*}$. In view of the reference model (7.1.6), matching conditions (7.3.2) and (7.3.8), and the closed-loop system (7.3.9), and ignoring the exponentially decaying terms, we obtain the output tracking error:

$$
\Delta e(k)=W_{m}(z) K_{p}\left[\widetilde{\Theta}^{T} \omega\right](k)
$$

where $\omega(k)=\left[\Delta x^{T}(k), r^{T}(k), 1\right]^{T}, \widetilde{\Theta}(k)=\Theta(k)-\Theta^{*}, \Theta(k)=\left[K_{1}^{T}(k), K_{2}(k), K_{3}(k)\right]^{T}$, and $\Theta^{*}=\left[K_{1}^{* T}, K_{2}^{*}, K_{3}^{*}\right]^{T}$. To deal with the uncertainty of $K_{p}$, we use its LDS decomposition (2.3.5):

$$
K_{p}=L_{s} D_{s} S
$$

where $S$ is symmetric positive definite, $L_{s}$ is unit lower triangular, and

$$
D_{s}=\operatorname{diag}\left\{\operatorname{sign}\left[\Delta_{1}\right] \gamma_{1}, \operatorname{sign}\left[\frac{\Delta_{2}}{\Delta_{1}}\right] \gamma_{2}, \ldots, \operatorname{sign}\left[\frac{\Delta_{M}}{\Delta_{M-1}}\right] \gamma_{M\}}\right.
$$

with $\gamma_{i}>0, i=1, \ldots, M$, arbitrarily chosen. It is worth noting that, from the design condition (A3), there exists an invariant $D_{s}$ for the piecewise constant $K_{p}$. 
Substituting the LDS decompensation of $K_{p}$ (with a uniform $D_{s}$ ) in (7.3.10), we have

$$
L_{s}^{-1} \xi_{m}(z)[\Delta e](k)=D_{s} S \widetilde{\Theta}^{T}(k) \omega(k)
$$

Operating both sides of $(7.3 .13)$ by $h(z)=1 / f_{h}(z)$, where $f_{h}(z)$ is a stable and monic polynomial of degree equals to the degree of $\xi_{m}(z)$, it leads to

$$
L_{s}^{-1} \xi_{m}(z) h(z)[e](k)=D_{s} S h(z)\left[\tilde{\Theta}^{T} \omega\right](k)
$$

Introducing $\Theta_{0}^{*}=L_{s}^{-1}-I=\theta_{i j}^{*}$ with $\theta_{i j}^{*}=0$ for $i=1,2, \ldots, M$ and $j \geq i,(7.3 .14)$ is parameterized as

$$
\bar{e}(k)+\left[0, \theta_{2}^{* T} \eta_{2}, \ldots, \theta_{M}^{* T} \eta_{M}\right]^{T}=D_{s} S h(z)\left[\tilde{\Theta}^{T} \omega\right](k),
$$

where

$$
\begin{aligned}
\bar{e}(k) & =\xi_{m}(z) h(z)[e](k)=\left[\bar{e}_{1}(k), \ldots, \bar{e}_{M}(k)\right]^{T}, \\
\eta_{i}(k) & =\left[\bar{e}_{1}(k), \ldots, \bar{e}_{i-1}(k)\right]^{T}, i=2, \ldots, M \\
\theta_{i}^{*} & =\left[\theta_{i 1}^{*}, \ldots, \theta_{i i-1}^{*}\right]^{T}, i=2, \ldots, M
\end{aligned}
$$

Estimation error. Introduce the estimation error

$$
\epsilon(k)=\left[0, \theta_{2}^{T}(k) \eta_{2}, \ldots, \theta_{M}^{T}(k) \eta_{M}\right]^{T}+\Psi(k) \xi(k)+\bar{e}(k),
$$

where $\theta_{i}(k), i=2, \ldots, M$ are the estimates of $\theta_{i}^{*}, \Psi$ is the estimate of $\Psi^{*}=D_{s} S$, and

$$
\xi(k)=\Theta^{T}(k) \zeta(k)-h(z)\left[\Theta^{T} \omega\right](k), \zeta(k)=h(z)[\omega](k) .
$$

From (7.3.15)-(7.3.20), we derive that

$$
\begin{aligned}
\epsilon(k)= & {\left[0, \tilde{\theta}_{2}^{T}(k) \eta_{2}(k), \tilde{\theta}_{3}^{T}(k) \eta_{3}(k), \ldots, \tilde{\theta}_{M}^{T}(k) \eta_{M}(k)\right]^{T} } \\
& +D_{s} S \tilde{\Theta}^{T}(k) \zeta(k)+\tilde{\Psi}(k) \xi(k)
\end{aligned}
$$


where $\tilde{\theta}_{i}(k)=\theta_{i}(k)-\theta_{i}^{*}, i=2,3, \ldots, M$, and $\tilde{\Psi}(k)=\Psi(k)-\Psi^{*}$ are the related parameter errors.

Adaptive laws. With the estimation error model (7.3.21), we choose the adaptive laws as

$$
\begin{aligned}
\theta_{i}(k+1) & =\theta_{i}(k)-\frac{\Gamma_{\theta i} \epsilon_{i}(k) \eta_{i}(k)}{m^{2}(k)}, i=2,3, \ldots, M, \\
\Theta^{T}(k+1) & =\Theta^{T}(k)-\frac{D_{s} \epsilon(k) \zeta^{T}(k)}{m^{2}(k)}, \\
\Psi(k+1) & =\Psi(k)-\frac{\Gamma \epsilon(k) \xi^{T}(k)}{m^{2}(k)},
\end{aligned}
$$

where the signal $\epsilon=\left[\epsilon_{1}, \epsilon_{2}, \ldots, \epsilon_{M}\right]^{T}$ is computed from (7.3.19), $0<\Gamma_{\theta i}=\Gamma_{\theta i}^{T}<2 I_{i-1}$, $i=2, \ldots, M, 0<\Gamma=\Gamma^{T}<2 I_{M}, D_{s}$ is chosen to satisfy $0<D_{s} S D_{s}<2 I_{M}$, and

$$
m(k)=\left(1+\zeta^{T}(k) \zeta(k)+\xi^{T}(k) \xi(k)+\sum_{i=2}^{M} \eta_{i}^{T}(k) \eta_{i}(k)\right)^{1 / 2} .
$$

Lemma 7.3.1. The adaptive laws (7.3.22)-(7.3.24) ensure that

(i) $\theta_{i}(k) \in l_{\infty}, i=2,3, \ldots, M, \Theta(k) \in l_{\infty}, \Psi(k) \in l_{\infty}$, and $\frac{\epsilon(k)}{m(k)} \in l_{2} \cap l_{\infty}$;

(ii) $\theta_{i}(k+1)-\theta_{i}(k) \in l_{2} \cap l_{\infty}, i=2,3, \ldots, M, \Theta(k+1)-\Theta(k) \in l_{2} \cap l_{\infty}$, and $\Psi(k+1)-\Psi(k) \in l_{2} \cap l_{\infty}$.

$\underline{\text { Proof: }}$ Consider a positive definite function

$$
V=\sum_{i=2}^{M} \tilde{\theta}_{i}^{T} \Gamma_{\theta i}^{-1} \tilde{\theta}_{i}+\operatorname{tr}\left[\tilde{\Psi}^{T} \Gamma^{-1} \tilde{\Psi}\right]+\operatorname{tr}\left[\tilde{\Theta} S \tilde{\Theta}^{T}\right],
$$

which has a finite jump at $k_{i}, i=1,2, \ldots, N-1$ when damage occurs. Within each interval $\left(k_{i-1}, k_{i}\right), i=1,2, \ldots, N$ with $k_{0}=0$ and $k_{N}=\infty$, the time-increment of $V$

$$
\begin{aligned}
& V\left(\tilde{\theta}_{i}(k+1), \tilde{\Psi}(k+1), \tilde{\Theta}(k+1)\right)-V\left(\tilde{\theta}_{i}(k), \tilde{\Psi}(k), \tilde{\Theta}(k)\right) \\
\leq & -\alpha_{1} \frac{\epsilon^{T}(k) \epsilon(k)}{m^{2}(k)}
\end{aligned}
$$


for some constant $\alpha_{1}>0$. That is $V\left(k_{i}\right) \leq V\left(k_{i-1}\right)$. Since $V(k)$ has a finite jump at $k_{i}, i=1,2, \ldots, N-1$, we can conclude that $V(k)$ is bounded. Therefore, we have $\theta_{i}(k) \in l_{\infty}, \Theta(k) \in l_{\infty}, \Psi(k) \in l_{\infty}$, and $\frac{\epsilon(k)}{m(k)} \in l_{\infty}$. From (7.3.26), we have $\frac{\epsilon(k)}{m(k)} \in l_{2}$. Then, from the adaptive laws (7.3.22)-(7.3.24), we obtain $\theta_{i}(k+1)-\theta_{i}(k) \in l_{2} \cap l_{\infty}$, $\Theta(k+1)-\Theta(k) \in l_{2} \cap l_{\infty}$, and $\Psi(k+1)-\Psi(k) \in l_{2} \cap l_{\infty}$.

These properties allow us to prove the following theorem.

Theorem 7.3.1. The multivariable MRAC scheme with the state feedback control law (7.3.1) updated by the adaptive laws (7.3.22)-(7.3.24), when applied to the plant (7.1.5), guarantees the closed-loop signal boundedness and asymptotic output tracking: $\lim _{k \rightarrow \infty}\left(\Delta y(k)-\Delta y_{m}(k)\right)=0$, for any initial conditions.

Proof: The main proof ideas are that (i) a well-defined feedback system structure is ensured by the boundednesss of the controller parameters, (ii) a small feedbackloop gain is ensured by the $l_{2}$ properties of $\Theta(k+1)-\Theta(k), \theta_{i}(k+1)-\theta_{i}(k)$ and $\frac{\epsilon(k)}{m(k)}$, and (iii) a smooth tracking error $\Delta e(k)=\Delta y(k)-\Delta y_{m}(k)$ is ensured by a bounded $\Delta e(k+1)-\Delta e(k)$. The properties (i) and (ii) guarantee the closed-loop signal boundedness and an $l_{2}$ tracking error $\Delta e(k)$, and the last property (iii) leads to a convergent tracking error $\Delta e(k): \lim _{k \rightarrow \infty}\left(\Delta y(k)-\Delta y_{m}(k)\right)=0$. The detailed proof is given as follows.

For clarity of presentation of the proof, we omit the symbol " $\Delta$ " in the linearized system model (7.1.5) and consider the simple version:

$$
x(k+1)=A x(k)+B u(k)+f_{0}, y(k)=C x(x) .
$$

Then, we have the input-output expression as

$$
y(k)=G_{0}(z)[u](k)+C(z I-A)^{-1} f_{0}\left[u_{s}\right](k),
$$


where $G_{0}(z)=C(z I-A)^{-1} B$ and $u_{s}(k)$ is a unit step response. To prove the theorem, we need to express the closed-loop system in a feedback structure in terms of the system input and output signals $u(k)$ and $y(k)$. For $x(k) \in R^{n}$ and $y(k) \in R^{M}$, based on state observation theory, under the condition that $(A, C)$ is observable (it can be extended to the case when $(A, C)$ is detectable), we can express the system (7.3.27) in a full-order state observer form:

$$
x(k+1)=(A-L C) x(k)+B u(k)+L y(k)+f_{0},
$$

where $L \in R^{n \times M}$ is chosen to make the eigenvalues of $A-L C$ stable (inside the unit circle). Note that a reduced-order state estimator can also be used. Hence, it can be shown that

$$
\begin{aligned}
x(k)= & (z I-A+L C)^{-1} B[u](k)+(z I-A+L C)^{-1} L[y](k) \\
& +(z I-A+L C)^{-1} f_{0}\left[u_{s}\right](k)+\epsilon_{x}(k),
\end{aligned}
$$

where $\epsilon_{x}(k) \in R^{n}$ is an exponentially decaying vector signal. In view of the state feedback controller (7.3.1):

$$
u(k)=K_{1}^{T}(k) x(k)+K_{2}(k) r(k)+K_{3}(k),
$$

and ignoring the exponentially decaying term $\epsilon_{x}(k)$, we have the feedback controller (7.3.31) in terms of $u(k)$ and $y(k)$ as

$$
\begin{aligned}
u(k)= & \Psi_{1}^{T}(k) \omega_{1}(k)+\Psi_{2}^{T}(k) \omega_{2}(k)+K_{2}(k) r(k) \\
& +\Psi_{u_{s}}^{T}(k) \omega_{u_{s}}(k)+K_{3}(k),
\end{aligned}
$$

where $\Psi_{1}(k) \in R^{n M \times M}, \Psi_{2}(k) \in R^{n M \times M}$, and $\Psi_{u_{s}}(k) \in R^{n \times M}$, are some parameter estimates, and

$$
\begin{aligned}
\omega_{1}(k) & =F(z)[u](k), \omega_{2}(k)=F(z)[y](k), \\
\omega_{u_{s}}(k) & =F_{u_{s}}(z)\left[u_{s}\right](k)
\end{aligned}
$$


with $F(z)=\frac{A_{d}(z)}{\Lambda(z)}, F_{u_{s}}(z)=\frac{A_{u_{s}}(z)}{\Lambda(z)}, A_{d}(z)=\left[I_{M}, z I_{M}, \ldots, z^{n-1} I_{M}\right]^{T}, A_{u_{s}}(z)=$ $\left[1, z, \ldots, z^{n-1}\right]^{T}$, and $\Lambda(z)$ being the monic characteristic polynomial of the stable matrix $A-L C$.

Letting $d_{m}$ be the maximum degree of the modified interactor matrix $\xi_{m}(z)$ of $G(z)=C(z I-A)^{-1} B$, we introduce fictitious filters $H_{1}(z), K_{1}(z)$ defined from

$$
(z-1) H_{1}(z)=1-K_{1}(z), K_{1}(z)=\frac{\left(1-\frac{1}{a_{1}}\right)^{d_{m}} z^{d_{m}}}{\left(z-\frac{1}{a_{1}}\right)^{d_{m}}}
$$

where $a_{1}>0$ is chosen to be sufficiently large but finite. Denoting $h_{1}(k)$ as the impulse response functions of the transfer functions $H_{1}(z)$, we have the $l_{1}$ operator norm

$$
\left\|h_{1}(\cdot)\right\|_{1}=\frac{d_{m}}{a_{1}-1}, a_{1}>1
$$

To show (7.3.35), we express $H_{1}(z)$ as

$$
\begin{aligned}
& \frac{1}{a_{1}} \frac{1}{z-\frac{1}{a_{1}}}\left(1+\frac{\left(1-\frac{1}{a_{1}}\right) z}{z-\frac{1}{a_{1}}}+\cdots+\frac{\left(1-\frac{1}{a_{1}}\right)^{d_{m}-1} z^{d_{m}-1}}{\left(z-\frac{1}{a_{1}}\right)^{d_{m}-1}}\right) \\
= & \frac{1}{a_{1}} \frac{1}{z-\frac{1}{a_{1}}} \frac{1-\frac{\left(1-\frac{1}{a_{1}}\right)^{d_{m}} z^{d_{m}}}{\left(z-\frac{1}{a_{1}}\right)^{d_{m}}}}{1-\frac{\left(1-\frac{1}{a_{1}}\right) z}{z-\frac{1}{a_{1}}}} \\
= & \frac{1-\frac{\left(1-\frac{1}{a_{1}}\right)^{d_{m}} z^{d_{m}}}{\left(z-\frac{1}{a_{1}}\right)^{d_{m}}}}{z-1}=H_{1}(z) .
\end{aligned}
$$

We consider $f_{j}(k)=Z^{-1}\left[\frac{1}{z-\frac{1}{a_{1}}} \frac{\left(1-\frac{1}{a_{1}}\right)^{j} z^{j}}{\left(z-\frac{1}{a_{1}}\right)^{j}}\right], j=0,1, \ldots, d_{m}-1$, and its sum $g_{j}(k)=$ $\sum_{\tau=0}^{k} f_{j}(\tau)$. We see that $f_{j}(k) \geq 0, \forall k \geq 0$, as $f_{j}(k)$ is the convolution of $f_{j-1}(k)$ and $Z^{-1}\left[\frac{\left(1-\frac{1}{a_{1}}\right) z}{z-\frac{1}{a_{1}}}\right]$, which are nonnegative, $j=1, \ldots, d_{m}-1$. We also see that $\lim _{k \rightarrow \infty} g_{j}(k)$ exists and is finite, as $a_{1}>1$. Hence, with $G_{j}(z)=Z\left[g_{j}(k)\right]=\frac{z}{z-1} F_{j}(z)\left(\right.$ as $g_{j}(k)$ is the convolution of $f_{j}(k)$ and the unit step function $u_{s}(k)$ whose $z$-transform is $\left.\frac{z}{z-1}\right)$ 
and $F_{j}(z)=Z\left[f_{j}(k)\right]=\frac{1}{z-\frac{1}{a_{1}}} \frac{\left(1-\frac{1}{a_{1}}\right)^{j} z^{j}}{\left(z-\frac{1}{a_{1}}\right)^{j}}$, we have

$$
\begin{aligned}
\sum_{\tau=0}^{\infty} f_{j}(\tau) & =\lim _{k \rightarrow \infty} g_{j}(k)=\lim _{z \rightarrow 1}\left(1-\frac{1}{z}\right) G_{j}(z) \\
& =\lim _{z \rightarrow 1} F_{j}(z)=\frac{1}{1-\frac{1}{a_{1}}}=\frac{a_{1}}{a_{1}-1} .
\end{aligned}
$$

Then, we have that $\left\|h_{1}(\cdot)\right\|_{1}=\sum_{j=1}^{d_{m}} \frac{1}{a_{1}} \sum_{\tau=0}^{\infty} f_{j}(\tau)=\frac{d_{m}}{a_{1}-1}$ for $a_{1}>1$.

From the plant model: $y(k)=G_{0}(z)[u](k), \omega_{1}(k)$ in (7.3.33), $H_{1}(z)$ and $K_{1}(z)$ in (7.3.34), we obtain

$$
\begin{array}{cc}
F(z) G_{0}^{-1}(z) & {[y] \quad(k)=K_{1}^{-1}(z)\left[\omega_{1}-H_{1}(z)(z-1)\left[\omega_{1}\right]\right](k)} \\
+\quad F(z) G_{0}^{-1}(z) C(z I-A)^{-1} f_{0}\left[u_{s}\right](k) .
\end{array}
$$

Let $\omega_{1}(k)=F(z)[u](k)$ (where $\left.F(z)=\frac{A_{d}(z)}{\Lambda(z)}\right)$ have a controllable realization $\left(A_{1}, B_{1}\right)$, that is,

$$
z\left[\omega_{1}\right](k)=\left(A_{1}+I\right) \omega_{1}(k)+B_{1} u(k),
$$

where $A_{1}+I$ is a stable matrix. From (7.3.32), (7.3.37), and (7.3.38), we have

$$
\begin{aligned}
\omega_{1}(k)= & K_{1}(z) F(z) G_{0}^{-1}(z)[y](k)+H_{1}(z)\left[A_{1} \omega_{1}\right](k) \\
& +H_{1}(z) B_{1}\left[\Psi_{1}^{T} \omega_{1}+\Psi_{2}^{T}(\cdot) F(z)[y]+K_{2} r\right. \\
& \left.+\Psi_{u_{s}}^{T} \omega_{u_{s}}+K_{3}\right](k) \\
& -K_{1}(z) F(z) G_{0}^{-1}(z) C(z I-A)^{-1} f_{0}\left[u_{s}\right](k) .
\end{aligned}
$$

Since the filter $H_{1}(z)$ satisfies (7.3.35) and $\Theta_{1}(k)$ is bounded, there exists a constant $a_{1}^{0}>0$ such that

$$
T_{1}(z, k)=\left(I-H_{1}(z)\left(A_{1}+B_{1} \Psi_{1}^{T}(k)\right)\right)^{-1}
$$

is a stable and proper operator with a finite gain for any fixed $a_{1}>a_{1}^{0}$. For a finite and fixed $a_{1}>a_{1}^{0}$ and a new signal $\hat{y}(k) \triangleq \frac{\xi_{m}(z)}{z+a_{0}}[y](k)$ where $0<a_{0}<1$ is arbitrary, 
it follows from (7.3.39) that

$$
\omega_{1}(k)=G_{1}(z, \cdot)[\hat{y}](k)+b_{3}(k)
$$

where

$$
\begin{aligned}
G_{1}(z, k)= & T_{1}(z, k)\left(K_{1}(z) F(z)\left(z+a_{0}\right) G_{0}^{-1}(z) \xi_{m}^{-1}(z)\right. \\
& \left.+H_{1}(z) B_{1} \Psi_{2}^{T}(k)\left(z+a_{0}\right) F(z) \xi_{m}^{-1}(z)\right)
\end{aligned}
$$

is stable and proper with a finite gain and

$$
\begin{aligned}
b_{3}(k)= & T_{1}(z, \cdot)\left[H_{1}(z) B_{1}\left[K_{2} r+\Psi_{u_{s}}^{T} \omega_{u_{s}}+K_{3}\right]\right. \\
& \left.-K_{1}(z) F(z) G_{0}^{-1}(z) C(z I-A)^{-1} f_{0}\left[u_{s}\right]\right](k)
\end{aligned}
$$

is bounded. Consider the estimation error $\epsilon(k)$ given in (7.3.19):

$$
\epsilon(k)=\left[0, \theta_{2}^{T} \eta_{2}, \ldots, \theta_{M}^{T} \eta_{M}\right]^{T}+\Psi(k) \xi(k)+\bar{e}(k)
$$

From the definition of $\left[0, \theta_{2}^{T}(k) \eta_{2}, \ldots, \theta_{M}^{T}(k) \eta_{M}\right]^{T}$, we write

$$
\left[0, \theta_{2}^{T}(k) \eta_{2}, \ldots, \theta_{M}^{T}(k) \eta_{M}\right]^{T}=\Theta_{0}(k) \bar{e}(k)
$$

where $\Theta_{0}(k)$ is the estimate of $\Theta_{0}^{*}$, a lower triangular matrix with zero diagonal elements. Then, from (7.3.44), we have

$$
\bar{e}(k)=\left(I+\Theta_{0}(k)\right)^{-1}(\epsilon(k)-\Psi(k) \xi(k)) .
$$

From this equation, for $\hat{y}(k)=\frac{\xi_{m}(z)}{z+a_{0}}[y](k)$ defined above, we have

$$
\hat{y}(k)=\frac{1}{z+a_{0}}[r]+\frac{1}{h(z)\left(z+a_{0}\right)}\left[\left(I+\Theta_{0}\right)^{-1}(\epsilon-\Psi \xi)\right](k) .
$$

From (7.3.20), we denote $\xi(k)=\left[\xi_{1}(k), \ldots, \xi_{M}(k)\right]^{T}, \Theta(k)=\left[\theta_{1}(k), \ldots, \theta_{M}(k)\right]$ with $f_{h}(z)=z^{d_{m}}+a_{d_{m}-1} z^{d_{m}-1}+\cdots+a_{1} z+a_{0}$. Then, $\xi_{i}(k)=\theta_{i}^{T}(k) \zeta(k)-\frac{1}{f_{h}(z)}\left[\theta_{i}^{T} \omega\right](k)$, 
$i=1, \ldots, M$, which, from the discrete-time swapping lemma [84], is expressed as

$$
\begin{aligned}
\xi_{i}(k)= & \frac{z^{d_{m}-1}+\cdots+a_{2} z+a_{1}}{f(z)}\left[(z-1)\left[\theta_{i}^{T}\right] \frac{z}{f(z)}[\omega]\right](k) \\
& +\frac{z^{d_{m}-2}+\cdots+a_{2}}{f(z)}\left[(z-1)\left[\theta_{i}^{T}\right] \frac{z^{2}}{f(z)}[\omega]\right](k)+\cdots \\
& +\frac{z+a_{d_{m}-1}}{f(z)}\left[(z-1)\left[\theta_{i}^{T}\right] \frac{z^{d_{m}-1}}{f(z)}[\omega]\right](k) \\
& +\frac{1}{f(z)}\left[(z-1)\left[\theta_{i}^{T}\right] \frac{z^{d_{m}}}{f(z)}[\omega]\right](k),
\end{aligned}
$$

where $(z-1)\left[\theta_{i}^{T}\right](k)=\theta_{i}^{T}(k+1)-\theta_{i}^{T}(k), i=1, \ldots, M$. Finally, from (7.3.30), (7.3.41), (7.3.47), and (7.3.48) and the boundedness of parameter estimates, we have

$$
\begin{aligned}
\|\hat{y}(k)\| & \leq \beta_{1} \sum_{\tau=0}^{k-1} e^{-\alpha_{1}(k-\tau-1)} \bar{x}(\tau)\left(\sum_{w=0}^{\tau-1} e^{-\alpha_{2}(\tau-w-1)}\|\hat{y}(w)\|\right) \\
& +\bar{x}_{0}(k)
\end{aligned}
$$

where $\bar{x}(k)=\|\Theta(k+1)-\Theta(k)\|+\left\|\frac{\epsilon(k)}{m(k)}\right\|$, for some $\beta_{1}, \alpha_{1}, \alpha_{2}>0, \bar{x}_{0}(k)$ is bounded. Here we used the fact that $\frac{\|\zeta(k)\|}{m(k)}, \frac{\|\xi(k)\|}{m(k)}$ and $\frac{\|\eta(k)\|}{m(k)}$ are bounded. Using the discrete-time version of Lemma 2.3 in [84] and from Lemma 2, we can show that $\hat{y}(k)$ is bounded, so are $u(k), y(k)$, and all signals in the closed-loop system. From the estimation error $\epsilon(k)$ in $(7.3 .44),(7.3 .48)$, and Lemma 2, we have $\bar{e}(k) \in l_{2}$, which implies that $\lim _{k \rightarrow \infty} \bar{e}(k)=0$, where $\bar{e}(k)=h(z) \xi_{m}(z)[e](k)$ Since $\xi_{m}(z)$ has a stable inverse, it follows that $\lim _{k \rightarrow \infty} e(k)=0$, where $e(k)=y(k)-y_{m}(k)$.

Linearization approximation errors. The adaptive control design is developed based on the linearized system without considering linearization approximation errors. The approximation errors could be compensated when they are small and the approximate output tracking could be achieved in an average sense. However, the exact asymptotic output tracking might not take place, even if the perturbation signals are small, since the perturbations could lead to some residual errors, or even 
parameter drift or instability. To avoid parameter drift, robust modifications of the adaptive laws, such as parameter projection, switch- $\sigma$ modification, can be used.

\subsection{Application to the NASA GTM}

In this section, the developed discrete-time adaptive control scheme will be applied to the NASA generic transport model (GTM) to assess its effectiveness for control of the nonlinear continuous-time aircraft flight system with structural damage. Before applying the developed adaptive damage compensation scheme, we first investigate the invariance conditions (A3) and (A4) based on the generic aircraft system models before and after damage occurs, and then the assumptions (A1) and (A2) can be verified based on the numerical values from the GTM.

An aircraft model with structural damage. Consider the aircraft model (2.1.1)-(2.1.9) with $\delta f_{i}, i=1,2, \ldots, 6$, characterizing the structural variations under damage, for which the state vector signal is $x=\left[u_{b}, w_{b}, q_{b}, \theta, v_{b}, r_{b}, p_{b}, \phi, \psi\right]^{T}$. In this simulation study, we choose $\theta$ and $\psi$ as two output signals, such that the output vector signal is $y=C x=[\theta, \psi]^{T}$ with

$$
C=\left[\begin{array}{l}
C_{1} \\
C_{2}
\end{array}\right]=\left[\begin{array}{lllllllll}
0 & 0 & 0 & 1 & 0 & 0 & 0 & 0 & 0 \\
0 & 0 & 0 & 0 & 0 & 0 & 0 & 0 & 1
\end{array}\right]
$$

and only manipulate the elevator $d_{e}$ and the rudder $d_{r}$, while set the other system input signals as the constant operating point values, such that the control input vector signal is $u=\left[u_{1}, u_{2}\right]^{T}=\left[d_{e}, d_{r}\right]^{T}$. Then the aircraft model used in this simulation study can be expressed as

$$
\dot{x}=f(x)+\sum_{i=1}^{2} g_{i}(x) u_{i}, y=C x=\left[h_{1}(x), h_{2}(x)\right]^{T} .
$$




\subsubsection{Invariance of Infinite Zero Structure of the Aircraft System under Structural Damage}

For the aircraft model (7.4.2) before and after damage occurs, from the aircraft system equations (2.1.1)-(2.1.9) and the chosen output matrix $C$ in (7.4.1), we obtain that

$$
L_{g_{j}} h_{i}(x)=C_{i} g_{j}(x)=0
$$

for $i=1,2$, and $j=1,2$, and

$$
\alpha\left(x_{0}\right)=\left[\begin{array}{ll}
\left.C_{1} \frac{\partial f}{\partial x}\right|_{x_{0}} g_{1}\left(x_{0}\right) & \left.C_{1} \frac{\partial f}{\partial x}\right|_{x_{0}} g_{2}\left(x_{0}\right) \\
\left.C_{2} \frac{\partial f}{\partial x}\right|_{x_{0}} g_{1}\left(x_{0}\right) & \left.C_{2} \frac{\partial f}{\partial x}\right|_{x_{0}} g_{2}\left(x_{0}\right)
\end{array}\right]
$$

is non-singular. That is the relative degree of (7.4.2) is $\left\{\rho_{1}, \rho_{2}\right\}=\{2,2\}$ before and after damage occurs.

Invariance of interactor matrix. Since the relative degree of the nonlinear aircraft system is $\left\{\rho_{1}, \rho_{2}\right\}=\{2,2\}$, from Corollary 1 , for a small sampling interval $T$, the linearized discrete-time aircraft model (7.1.5) has the same interactor matrix: $\xi_{m}(z)=\operatorname{diag}\left\{z+a_{1}, z+a_{2}\right\}$, with $\left|a_{1}\right|<1$ and $\left|a_{2}\right|<1$, before and after damage.

Invariance of high frequency gain matrix. From the proof of Corollary 1 in the Appendix, we obtain the high frequency gain matrix (7.2.35) before and after damage occurs as

$$
K_{p} \approx\left[\begin{array}{cc}
\frac{T^{2}}{2} & 0 \\
0 & \frac{T^{2}}{2}
\end{array}\right]\left[\begin{array}{ll}
C_{1} A_{c} B_{c 1} & C_{1} A_{c} B_{c 2} \\
C_{2} A_{c} B_{c 1} & C_{2} A_{c} B_{c 2}
\end{array}\right],
$$

where $\left.A_{c} \triangleq \frac{\partial f}{\partial x}\right|_{x_{0}}$ and $\left[B_{c 1}, B_{c 2}\right]=B_{c} \triangleq\left[g_{1}\left(x_{0}\right), g_{2}\left(x_{0}\right)\right]$ are matrices of the linearized continuous-time system by linearizing the aircraft system (7.4.2) at the operating point $\left(x_{0}, 0\right)$. For the linearized continuous-time aircraft system $\left(A_{c}, B_{c}, C\right)$, it has been shown in [57] that, when operating at a wings-level flight condition, signs of leading principal minors of the high frequency gain matrix:

$$
K_{p c}=\left[\begin{array}{ll}
C_{1} A_{c} B_{c 1} & C_{1} A_{c} B_{c 2} \\
C_{2} A_{c} B_{c 1} & C_{2} A_{c} B_{c 2}
\end{array}\right]
$$


are invariant before and after damage occurs. Hence, from (7.4.5), we conclude that for the linearized discrete-time aircraft model (7.1.5), signs of leading principal minors of $K_{p}$ are invariant before and after damage occurs, when operating at the wings-level flight condition.

\subsubsection{Simulation Study for the NASA GTM}

The GTM is a $5.5 \%$ dynamically scaled twin-turbine powered test aircraft used to test flight research control laws in adverse flight conditions such as upsets, damage, and failures [64]. We use the high-fidelity Matlab Simulink model of the GTM developed by the NASA, which contains actuator dynamics, sensor dynamics, aerodynamics, etc., to test the developed control design. The nonlinear GTM simulation will offer a realistic representation of the aircraft and simulation results can provide a credible assessment of the developed design.

Damage scenarios. The GTM simulation model contains several damage scenarios. In this study, we consider two damage conditions:

(i) loss of outboard left wing tip (approximately 25\% semi-span of the left wing);

(ii) loss of entire left stabilizer.

Design conditions. The invariance assumptions (A3) and (A4) for the infinite zero structure have been verified by the generic structure study in Section 7.4.1. The assumptions (A1) and (A2) can be verified by the numerical values of the system parameters obtained from the GTM Simulink model.

Digital control of GTM. The block diagram of the digital control system framework is shown in Fig. 7.1. The operating point $\left(x_{0}, u_{0}\right)$ is chosen as a wings-level flight condition obtained by trimming the nominal GTM with the equivalent airspeed as 90 knots. The output signals are chosen as the pitch angle $\theta$ and the yaw angle $\psi$ : 
$y(k T)=[\theta(k T), \psi(k T)]^{T}$, and the control inputs are chosen as elevator $d_{e}$ and rudder $d_{r}: u(k T)=\left[d_{e}(k T), d_{r}(k T)\right]^{T}$. From the analysis in Section 7.4.1, we have the interactor matrix is invariant before and after damage occurs with a small sampling interval $T$, which is $\xi_{m}(z)=\operatorname{diag}\left\{z+a_{1}, z+a_{2}\right\}$ with $\left|a_{1}\right|<1$ and $\left|a_{2}\right|<1$. Hence, we choose the reference system as

$$
W_{m}(z)=\xi_{m}^{-1}(z)=\operatorname{diag}\{1 / z, 1 / z\}
$$

for the simulation study.

By applying the discrete-time control law $u(k)=\Delta u(k)+u_{0}$, where $\Delta u(k)$ is the adaptive controller (7.3.1) with the adaptive laws (7.3.22)-(7.3.24), to the continuoustime GTM via the ZOHs (illustrated in Fig. 7.1), we can obtain the desired system performance of the nonlinear GTM around the chosen operating point $\left(x_{0}, u_{0}\right)$, before and after damage occurs.

Simulation results. In addition to show the output signal $y(k T)=\Delta y(k T)+$ $C x_{0}=\left[\theta(k T)+\theta_{0}, \psi(k T)+\psi_{0}\right]^{T}$, another state signal-roll angle $\phi(k T)=\Delta \phi(k T)+\phi_{0}$, and the control surfaces $d_{e}(k T)=\Delta d_{e}(k T)+d_{e 0}$ and $d_{r}(k T)=\Delta d_{r}(k T)+d_{r 0}$, will be illustrated to verify that the aircraft can execute the maneuvers around the chosen operating point $\left(x_{0}, u_{0}\right)$. We consider two damage cases: the loss of the outboard left wing-tip and the loss of the entire left stabilizer.

Case I. We choose the sampling interval $T=0.02$ seconds and the reference input as $r(k T)=[4 \pi / 180 \sin (0.1 k T),-8 \pi / 180 \sin (0.15 k T)]^{T}$. The wing-tip damage occurs at 30 seconds. From Fig. 7.2, it can be seen that the output signals (solid)-pitch angle $\theta(k T)$ and yaw angle $\psi(k T)$ track the reference output signals (dotted) $\theta_{m}(k T)$ and $\psi_{m}(k T)$, and the GTM state signal-roll angle $\phi(k T)$ is bounded before and after damage occurs. Moreover, the controller positions $-d_{e}(k T)$ and $d_{r}(k T)$ are within the GTM limits as in Fig. 7.3. 

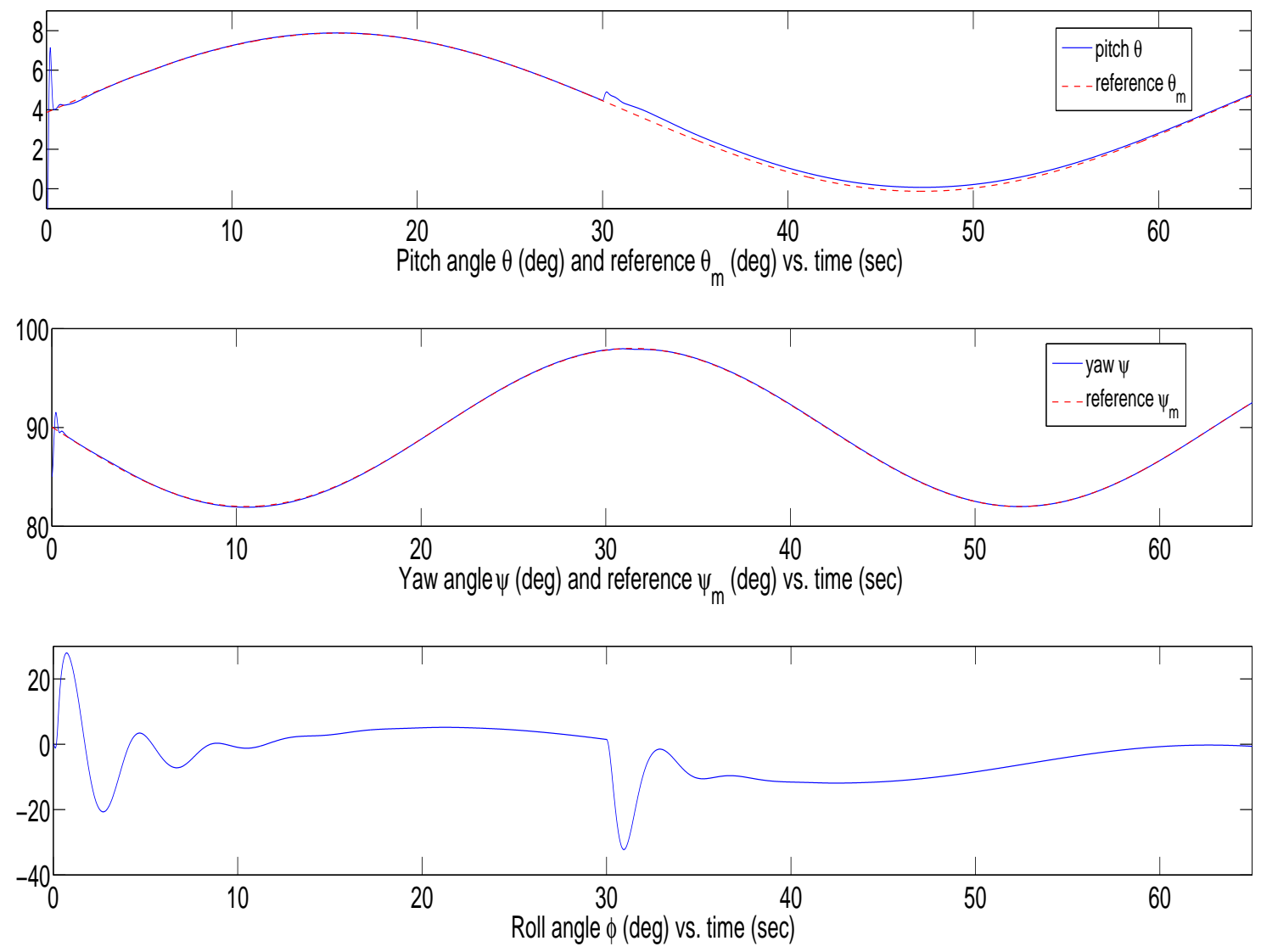

Figure 7.2: pitch $\theta(k T)$, yaw $\psi(k T)$, and roll $\phi(k T)$ (Case I). 

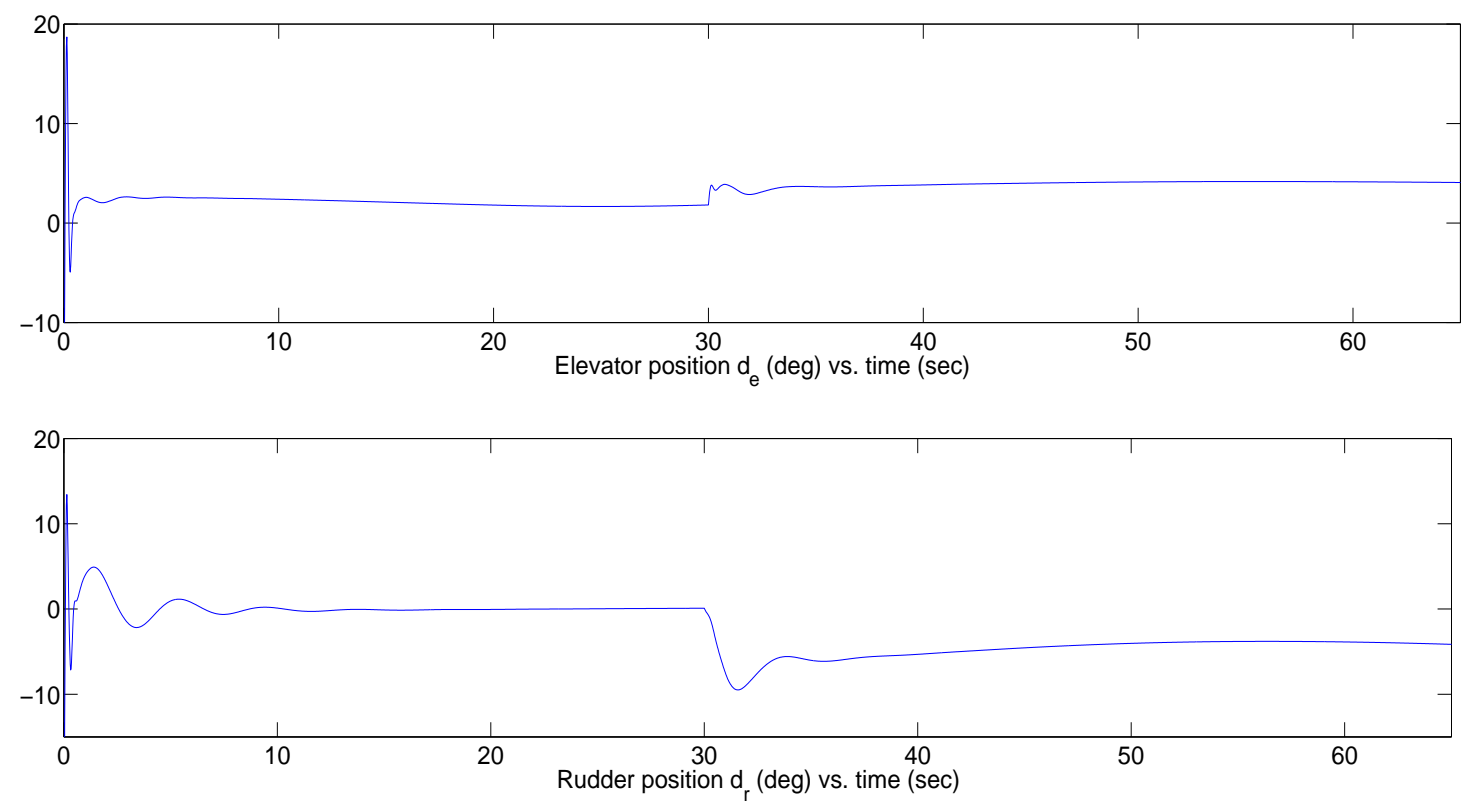

Figure 7.3: elevator $d_{e}(k T)$ and rudder $d_{r}(k T)$ (Case I).

Case II. To further demonstrate the effectiveness of the developed discrete-time control scheme, damage is chosen as the loss of the entire left stabilizer, which occurs at 20 seconds. In the simulation, we choose the sampling interval $T=0.05$ seconds and the reference input as $r(k T)=[4 \pi / 180,6 \pi / 180]^{T}$. Fig. 7.4 shows the GTM output signals $\theta(k T)$ and $\psi(k T)$, which track the desired reference signals, and the GTM state signal $\phi(k T)$, which is bounded within a reasonable boundary. The control surface positions $d_{e}(k T)$ and $d_{r}(k T)$ are shown in Fig. 7.5.

Remark 7.4.1. During the transient period after damage occurs, the adaptive controller automatically adjusts its parameters to accommodate the unknown system dynamics variations due to damage. So the transient responses may highly depend on variations of system model dynamics caused by structural damage. For the simulation studies of Case I and Case II, the sampling time $T$ is small ( $T=0.02 \mathrm{sec}$ for Case I and $T=0.05 \mathrm{sec}$ for Case II), which leads to small numerical values for parameters in 

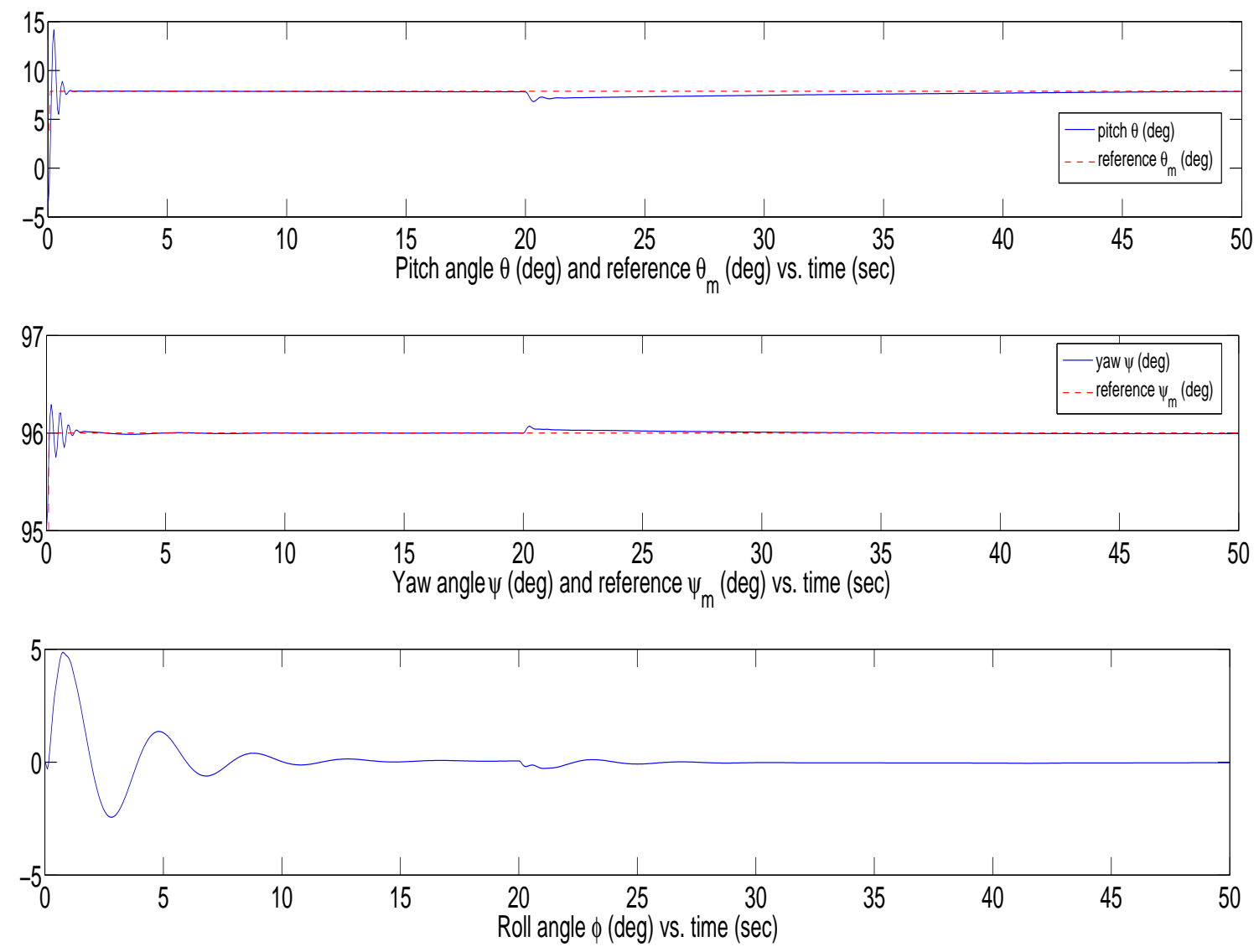

Figure 7.4: pitch $\theta(k T)$, yaw $\psi(k T)$, and roll $\phi(k T)$ (Case II). 

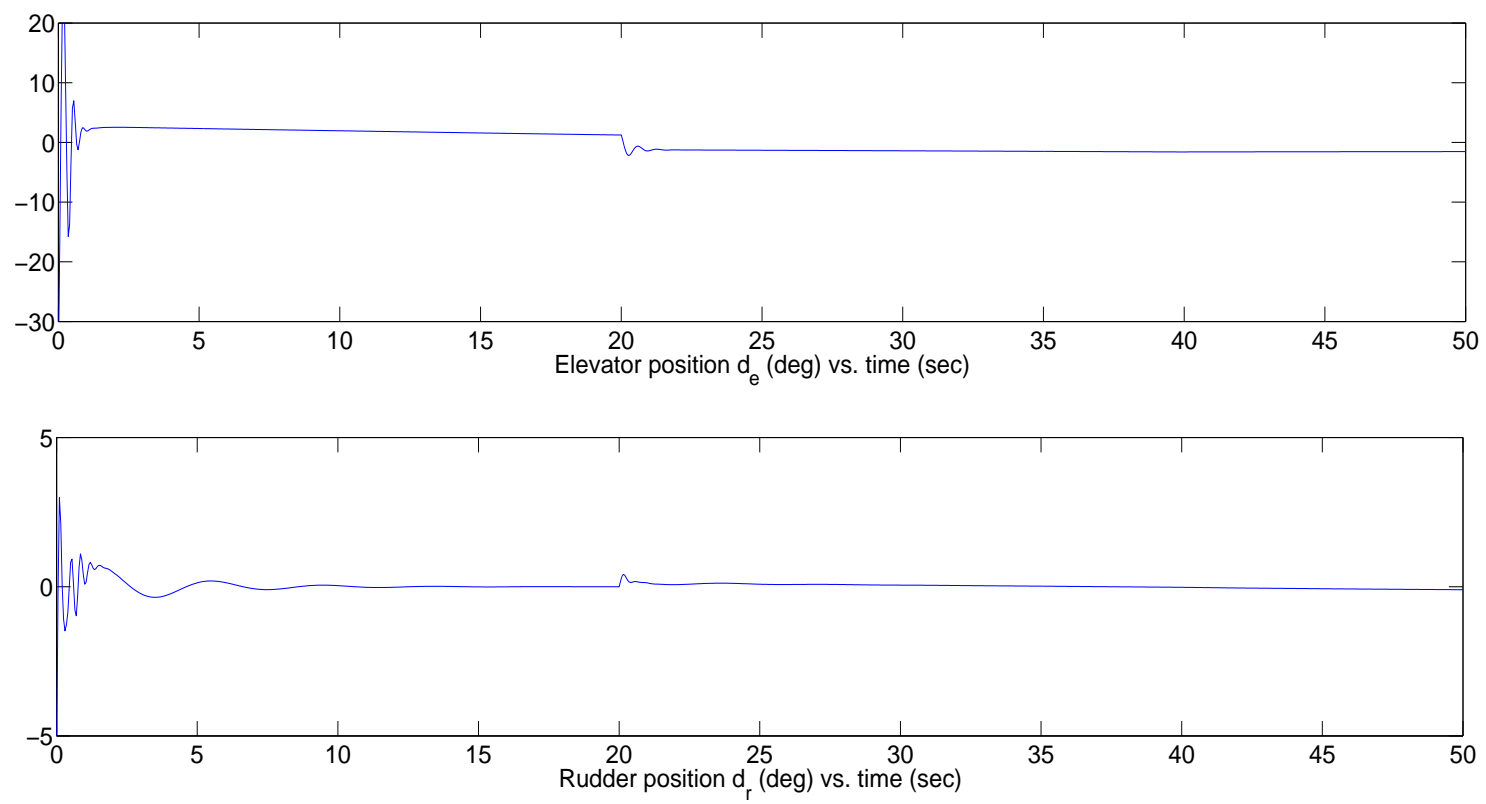

Figure 7.5: elevator $d_{e}(k T)$ and rudder $d_{r}(k T)$ (Case II).

$A$ and $B$ of the linearized discretized aircraft model. It follows that parametric variations of the linearized discretized aircraft model before and after damage occurs are small in the sense of numerical values, so that the transient responses after damage are small in Case I and Case II.

\section{Summary}

In this chapter, a digital control system framework for control of continuous-time nonlinear systems with possible structural damage was constructed, with an aircraft flight control application to the NASA GTM. In this framework, a linearization-based discrete-time multivariable model reference adaptive control (MRAC) scheme is applied to compensate the parameter and damage uncertainties. For control design, we obtained the discretized nonlinear system by using the Taylor series expansion, and then linearized the discrete-time nonlinear model at a given operating point to 
obtain a linearized discrete-time system. The infinite zero structure of the linearized discrete-time system model of the aircraft system is shown to be invariant, which ensures that the plant-model matching condition holds before and after damage occurs. With the achievable plant-model matching condition, the developed discrete-time MRAC design makes the signals of the closed-loop system bounded and the output signals track reference signals before and after damage occurs. Simulation results of the proposed digital control design framework for the nonlinear NASA generic transport model (GTM) have shown the desired system performance, which demonstrates that the linearization-based discrete-time adaptive control scheme is effective for the continuous-time nonlinear aircraft system around an operating point. 


\section{Chapter 8}

\section{Adaptive Output Feedback Actuator Nonlinearity Compensation for MIMO Systems}

This chapter develops a framework of adaptive compensation of actuator nonlinearities with unknown parameters, for output feedback control of unknown MIMO linear time-invariant dynamic systems. A new controller parametrization is derived to deal with bilinear parameters from the actuator nonlinearity parameters and the dynamic system parameters, which, based on the LDU decomposition of the system high frequency gain matrix, is capable of overcoming the difficulty caused by a nondiagonal high frequency gain matrix and a special actuator nonlinearity parameter structure. The adaptive actuator nonlinearity compensation control scheme is a model reference adaptive control based design, employing an adaptive output feedback control law combined with an adaptive actuator nonlinearity inverse, to deal with parameter uncertainties in the system dynamics and actuator nonlinearities. Simulation results show the desired adaptive control system performance. 


\subsection{Problem Statement}

Consider a multi-input multi-output control system

$$
\dot{x}(t)=A x(t)+B u(t), u(t)=N(v(t)), y(t)=C x(t),
$$

with $A \in R^{n \times n}, B \in R^{n \times M}$ and $C \in R^{M \times n}$ being unknown and constant parameter matrices, and $x(t) \in R^{n}, v(t) \in R^{M}$ and $y(t) \in R^{M}$ being the system state, control and output vector signals, where $N(\cdot)$ is the actuator nonlinearity and the input vector signal $u(t) \in R^{M}$ is not accessible for control and measurement. Such a system can be expressed in the input-output form:

$$
y(t)=G(s)[u](t), \quad u(t)=N(v(t))
$$

where $G(s)$ is an $M \times M$ strictly proper rational matrix $G(s)=C(s I-A)^{-1} B$ and $u(t)=\left[u_{1}(t), \ldots, u_{M}(t)\right]^{T}$ and $v(t)=\left[v_{1}(t), \ldots, v_{M}(t)\right]^{T}$ are the output and input of the multivariable actuator nonlinearity $N(\cdot)=\left[N_{1}(\cdot), \ldots, N_{M}(\cdot)\right]^{T}$, that is,

$$
u_{i}(t)=N_{i}\left(v_{i}(t)\right), i=1,2, \ldots, M
$$

for some nonlinear functions $N_{i}(\cdot)$ such as deadzone, backlash or hysteresis.

Control objective. The control objective is to generate the control signals $v_{i}(t)$ to cancel the effects of the nonlinearities $N_{i}(\cdot)$, using adaptive inverses $v_{i}=\widehat{N I}_{i}\left(u_{d i}\right)$ of the nonlinear characteristics $N_{i}(\cdot), i=1, \ldots, M$, to be combined with a commonly used multivariable control scheme which generates the feedback control signals $u_{d i}(t)$ designed for unknown $G(s)$, to ensure all signals in the closed-loop system are bounded and the system output $y(t)$ tracks the reference signal $y_{m}(t)$ :

$$
y_{m}(t)=W_{m}(s)[r](t), W_{m}(s)=\xi_{m}^{-1}(s)
$$

where $r(t)$ is a chosen reference input signal. 
Assumptions. To proceed the adaptive control design, we make the following assumptions for the unknown transfer matrix $G(s)=C(s I-A)^{-1} B$ : $(\mathrm{A} 1)(A, B)$ is controllable and $(A, C)$ is observable, (A2) $G(s)$ has full rank and a known modified interactor matrix $\xi_{m}(s)$, (A3) all zeros of $G(s)$ are stable, (A4) the observability index $\nu$ (or its upper bound) of $G(s)$ is known, and (A5) all leading principal minors of the high frequency gain matrix $K_{p}$ are nonzero and their signs are known.

Actuator nonlinearity model and its inverse model. Consider the cases when $N_{i}(\cdot)$ can be parameterized as

$$
u_{i}(t)=N_{i}\left(v_{i}(t)\right)=-\theta_{N i}^{* T} \omega_{N i}^{*}(t)+a_{i}^{*}(t)
$$

for some unknown parameter vectors $\theta_{N i}^{*} \in R^{n_{i}}, n_{i} \geq 1, i=1, \ldots, M$, and some unknown regressor vector signals $\omega_{N i}^{*}(t) \in R^{n_{i}}$ and scalar signals $a_{i}^{*}(t)$. Such a parametrization has been established for $N_{i}(\cdot)$ being a dead-zone, backlash, hysteresis, or other characteristics [83], [84]. To cancel the effects of such actuator nonlinearities, we use a multivariable nonlinearity inverse

$$
v(t)=\widehat{N I}\left(u_{d}(t)\right)
$$

where $u_{d}(t)=\left[u_{d 1}(t), \ldots, u_{d M}(t)\right]^{T}$ is a design vector signal from a feedback control law, that is,

$$
v_{i}(t)=\widehat{N I}_{i}\left(u_{d i}(t)\right), i=1, \ldots, M
$$

Then, each $\widehat{N I}_{i}(\cdot)$ can be parametrized as

$$
u_{d i}(t)=-\theta_{N i}^{T}(t) \omega_{N i}(t)+a_{i}(t), i=1, \ldots, M
$$

where $\theta_{N i} \in R^{n_{i}}$ is an estimate of $\theta_{N i}^{*}$ and $\omega_{N i}(t) \in R^{n_{i}}$ and $a_{i}(t)$ are some known signals, as in the case of an inverse for a dead-zone, backlash, or hysteresis [83], [84]. 
The uncertainties in $N_{i}(\cdot)$ cause a control error

$$
u_{i}(t)-u_{d i}(t)=\tilde{\theta}_{N i}^{T}(t) \omega_{N i}(t)+d_{N i}(t), i=1, \ldots, M
$$

where $\tilde{\theta}_{N i}=\theta_{N i}-\theta_{N i}^{*}$ and the unparameterized error is

$$
d_{N i}(t)=\theta_{N i}^{* T}\left(\omega_{N i}(t)-\omega_{N i}^{*}(t)\right)+a_{i}^{*}(t)-a_{i}(t)
$$

which should satisfy that conditions that $d_{N i}(t)$ is bounded, $t \geq 0$, and $d_{N i}(t)=0$, $t \geq t_{0}$, if $\theta_{N i}(t)=\theta_{N i}^{*}, t \geq t_{0}$, and $\widehat{N I}_{i}(\cdot)$ is correctly initialized: $d_{N i}\left(t_{0}\right)=0$. In the vector form, the control error is

$$
u(t)-u_{d}(t)=\tilde{\Theta}_{N}^{T}(t) \omega_{N}(t)+d_{N}(t)
$$

where $\omega_{N}(t)=\left[\omega_{N 1}^{T}(t), \ldots, \omega_{N M}^{T}(t)\right]^{T}$ and

$$
\tilde{\Theta}_{N}^{T}(t)=\operatorname{diag}\left\{\tilde{\theta}_{N 1}^{T}(t), \tilde{\theta}_{N 2}^{T}(t), \ldots, \tilde{\theta}_{N M}^{T}(t)\right\}
$$

Synthetic jet actuator model and its inverse. A synthetic jet actuator is a zero-net mass flux device that produces non-zero fluid momentum across an orifice. At certain operation condition, the synthetic jet actuator can be modeled by a nonlinear function [18]:

$$
u(t)=N(v(t))=\theta_{2}^{*}-\frac{\theta_{1}^{*}}{v(t)}=-\theta_{N}^{* T} \omega_{N}(t),
$$

where $u(t)$ is the equivalent virtual deflection on the airfoil, $v(t)=A_{p p}^{2}(t)$ with $A_{p p}(t)$ being the input peak-to-peak voltage amplitude of the synthetic jet, $\theta_{1}^{*}$ and $\theta_{2}^{*}$ are some unknown constant parameters, and $\theta_{N}^{*}=\left[\theta_{1}^{*}, \theta_{2}^{*}\right]^{T}$ and $\omega_{N}(t)=\left[\frac{1}{v(t)},-1\right]^{T}$. In practice, there are opposite panels to produce negative commanded control input signal $v(t)$. From (8.1.13), to cancel the effects of the unknown nonlinearity, we use the adaptively updated local inverse:

$$
v(t)=\widehat{N I}\left(u_{d}(t)\right)=\frac{\theta_{1}(t)}{\theta_{2}(t)-u_{d}(t)},
$$


where $\theta_{1}(t)$ and $\theta_{2}(t)$ are the estimates of $\theta_{1}^{*}$ and $\theta_{2}^{*}$ and $u_{d}(t)$ is the design signal from the feedback control law, such that $\theta_{2}-u_{d}>0$. Then, the synthetic jet actuator nonlinearity inverse (8.1.14) can be parameterized as $u_{d}(t)=\theta_{2}(t)-\frac{\theta_{1}(t)}{v(t)}=-\theta_{N}^{T}(t) \omega_{N}(t)$ with $\theta_{N}(t)=\left[\theta_{1}(t), \theta_{2}(t)\right]^{T}$ and $\omega_{N}(t)=\left[-\frac{1}{v(t)}, 1\right]^{T}$.

Since the parameters $\theta_{N i}^{*}$ of the actuator nonlinearities $N_{i}(\cdot)$ are unknown in our adaptive control problems, we need to design adaptive schemes to update their estimates $\theta_{N i}$ for implementing the adaptive inverses $\widehat{N I}_{i}(\cdot)$, to be combined with feedback control laws for the case when $G(s)$ is unknown.

Key technical issues. A key technical issue in adaptive control of multivariable systems with uncertain actuator nonlinearities is parametrization of the bilinear parameters in the system high frequency gain matrix $K_{p}$ and the actuator nonlinearity parameter vectors $\theta_{N i}^{*}, i=1,2, \ldots, M$. The technical difficulty was illustrated in [88] for the simple case when $y(t)=G(s)[u](t)$ with $G(s)=W_{m}(s) K_{p}$, where $W_{m}(s)$ is known and stable and $K_{p}$ is nonsingular and unknown. Without special treatments, adaptive laws may not be able to generate $\Theta_{N}(t)$ to have the required special form (8.1.12) in which all of the off block-diagonal elements of $\Theta_{N}$ need to be zero and should not be updated, for the implementation of individual adaptive inverses $\widehat{N I}(\cdot)$, $i=1,2, \ldots, M$. In this chapter, we propose to use the LDU decomposition of $K_{p}$ as in (2.3.3) to solve this issue.

\subsection{Adaptive Actuator Nonlinearity Compensation}

In this section we develop a general framework for the design of adaptive actuator nonlinearity compensation control schemes which employ an adaptive inverse $\widehat{N I}(\cdot)$ to handle the actuator nonlinearity $N(\cdot)$ and an adaptive feedback control law $u_{d}$ to handle $G(s)$. We will first use a plant-model matching condition to derive an error 
signal identity, based on which the output feedback adaptive controller structure will be developed.

Plant-model matching condition. For the system transfer matrix $G(s)=$ $C(D I-A)^{-1} B$, there exist constant parameters $\Theta_{1}^{*}, \Theta_{2}^{*}, \Theta_{20}^{*}$, and $\Theta_{3}^{*}$, such that $[84]$

$$
\begin{aligned}
& I_{M}-\Theta_{1}^{* T} F(s)-\left(\Theta_{2}^{* T} F(s)+\Theta_{20}^{*}\right) G(s) \\
= & \Theta_{3}^{*} \xi_{m}(s) G(s), \quad \Theta_{3}^{*}=K_{p}^{-1},
\end{aligned}
$$

with $F(s)=\frac{A_{0}(s)}{\Lambda(s)}$, where $\Lambda(s)$ is a monic and stable polynomial of degree $\nu-1$ and $A_{0}(s)=\left[I_{M}, \ldots, s^{\nu-2} I_{M}\right]^{T}$ with $\nu$ being the observability index.

Tracking error identity. Operating both sides of (8.2.1) on $u(t)$ and from $y(t)=G(s)[u](t)$, we obtain

$$
u(t)-\Theta_{1}^{* T} \omega_{1}-\Theta_{2}^{* T} \omega_{2}-\Theta_{20}^{*} y(t)=K_{p}^{-1} \xi_{m}(s)[y](t),
$$

where $\omega_{1}(t)=F(s)[u](t)$ and $\omega_{2}=F(s)[y](t)$. Hence, from the reference system (8.1.4) and the equation (8.2.2), we have the tracking error $e(t)=y(t)-y_{m}(t)$ as

$$
e(t)=W_{m}(s) K_{p}\left[u-\Theta_{1}^{* T} \omega_{1}-\Theta_{2}^{* T} \omega_{2}-\Theta_{20}^{*} y-\Theta_{3}^{*} r\right](t) .
$$

\subsubsection{Adaptive Controller Parametrization}

For the system (8.1.2) with actuator nonlinearities $u(t)=N(v(t))$, we will employ the nonlinearity inverse $v(t)=\widehat{N I}\left(u_{d}(t)\right)$ with $\widehat{N I}(\cdot)$ parameterized as (8.1.8):

$$
u_{d}(t)=\Theta_{N}^{T}(t) \omega_{N}(t)+a_{N}(t)
$$

to cancel the effect of the actuator nonlinearities, where the design commanded signal $u_{d}(t)$ will be developed based on the output feedback adaptive control design framework. Next, we will present a new parametrization of the controller $u_{d}(t)$ based on the tracking error equation (8.2.3) with the LDU decomposition of $K_{p}$. 
Design based on LDU decomposition. Substituting the LDU decomposition (2.3.3) of $K_{p}$ in (8.2.3), we obtain

$$
e=W_{m}(s) L D^{*}\left[u-\Phi_{0}^{*} u-\Phi_{1}^{* T} \omega_{1}-\Phi_{2}^{* T} \omega_{2}-\Phi_{20}^{*} y-\Phi_{3}^{*} r\right](t)
$$

where $\Phi_{0}^{*}=I-U, \Phi_{i}^{*}=U \Theta_{i}^{*}$, for $i=1,2,20,3$. In view of (8.1.11), we have

$$
u(t)=u_{d}(t)+\widetilde{\Theta}_{N}^{T}(t) \omega_{N}(t)+d_{N}(t)
$$

and then from (8.2.4), $u(t)$ can also be expressed as

$$
u(t)=-\Theta_{N}^{* T} \omega_{N}(t)+a_{N}(t)+d_{N}(t)
$$

Replacing the first $u(t)$ in the right-hand side of (8.2.5) by (8.2.6) and the second and third $u(t)$ (in $\left.\omega_{1}(t)\right)$ with $(8.2 .7)$, we obtain

$$
\begin{aligned}
& e=W_{m}(s) L D^{*}\left[u_{d}+\widetilde{\Theta}_{N}^{T} \omega_{N}+d_{N}-\Phi_{0}^{*}\left(-\Theta_{N}^{* T} \omega_{N}+a_{N}+d_{N}\right)\right. \\
& \left.-\Phi_{1}^{* T} F(s)\left[-\Theta_{N}^{* T} \omega_{N}+a_{N}+d_{N}\right]-\Phi_{2}^{* T} \omega_{2}-\Phi_{20}^{*} y-\Phi_{3}^{*} r\right](t) .
\end{aligned}
$$

With $\Phi_{0}^{*}$ in the special structure: $\Phi_{0}^{*}=\left\{\phi_{i j}^{*}\right\}$ where $\phi_{i j}^{*}=0$ for $i=1,2, \ldots, M$, and $j \leq i$, we have

$$
\Phi_{0}^{*}\left(-\Theta_{N}^{* T} \omega(t)+a_{N}\right)=\left[\phi_{\theta 1}^{* T} \omega_{\theta 1}, \ldots, \phi_{\theta M-1}^{* T} \omega_{\theta M-1}, 0\right]^{T}
$$

where the new parameter vectors are $\phi_{\theta 1}^{*}=\left[-\phi_{12}^{*} \theta_{N 2}^{* T}, \ldots,-\phi_{1 M}^{*} \theta_{N M}^{* T}, \phi_{12}^{*}, \ldots, \phi_{1 M}^{*}\right]^{T}$, $\phi_{\theta 2}^{*}=\left[-\phi_{23}^{*} \theta_{N 3}^{* T}, \ldots,-\phi_{2 M}^{*} \theta_{N M}^{* T}, \phi_{23}^{*}, \ldots, \phi_{2 M}^{*}\right]^{T}, \ldots, \phi_{\theta M-1}^{*}=\left[-\phi_{M-2 M}^{*} \theta_{N M}^{* T}, \phi_{M-2 M}^{*}\right]^{T}$, and the new regressor signals are $\omega_{\theta 1}(t)=\left[\omega_{N 2}^{T}(t), \ldots, \omega_{N M}^{T}(t), a_{2}(t), \ldots, a_{M}(t)\right]^{T}$, $\omega_{\theta 2}(t)=\left[\omega_{N 3}^{T}(t), \ldots, \omega_{N M}^{T}(t), a_{3}(t), \ldots, a_{M}(t)\right]^{T}, \ldots, \omega_{\theta M-1}(t)=\left[\omega_{N M}^{T}(t), a_{M}(t)\right]^{T}$. Similarly but in a new framework, we have

$$
\Phi_{1}^{* T} F(s)\left[-\Theta_{N}^{* T} \omega_{N}+a_{N}\right](t)=\Phi_{4}^{* T} \omega_{4}(t)+\Phi_{40}^{* T} \omega_{40}(t)
$$


where $\omega_{40}(t)=F(s)\left[a_{N}\right](t), \Phi_{40}^{*}=\Phi_{1}^{*}$, and, for $\Phi_{1}^{* T}=\left[\Phi_{11}^{* T}, \Phi_{12}^{* T}, \ldots, \Phi_{1 \nu-1}^{* T}\right]$ with $\Phi_{1 i}^{*} \in R^{M \times M}$, the new parameter matrix $\Phi_{4}^{* T}=\Phi_{1}^{* T} \otimes \Theta_{N}^{*}$ (with $\otimes$ denoting the Kronecker product) and the new regressor vector $\omega_{4}(t)$ are $\Phi_{4}^{* T}=-\left[\Phi_{11}^{* T} \Theta_{N}^{* T}, \ldots, \Phi_{1 \nu-1}^{* T} \Theta_{N}^{* T}\right]$ and $\omega_{4}(t)=\frac{A_{\theta}(s)}{\Lambda(s)}\left[\omega_{N}\right](t)$ with $A_{\theta}=\left[I_{n_{\theta}}, s I_{n_{\theta}}, \ldots, s^{\nu-2} I_{n_{\theta}}\right]^{T}$ and $I_{n_{\theta}}$ being the $n_{\theta} \times n_{\theta}$ identity matrix for $n_{\theta}=\sum_{i=1}^{M} n_{i}$. Then, from (8.2.9) and (8.2.10), the error equation (8.2.8) can be parameterized as

$$
\begin{aligned}
e(t) & =W_{m}(s) L D^{*}\left[u_{d}+\tilde{\Theta}_{N}^{T} \omega_{N}-\left[\phi_{\theta 1}^{* T} \omega_{\theta 1}, \ldots, \phi_{\theta M-1}^{* T} \omega_{\theta M-1}, 0\right]^{T}\right. \\
& \left.-\Phi_{4}^{* T} \omega_{4}-\Phi_{40}^{*} \omega_{40}-\Phi_{2}^{* T} \omega_{2}-\Phi_{20}^{*} y-\Phi_{3}^{*} r+d_{N f}\right](t),
\end{aligned}
$$

where $d_{N f}(t) \triangleq\left(I_{M}-\Phi_{0}^{*}-\Phi_{1}^{* T} F(s)\right)\left[d_{N}\right](t)$.

Adaptive controller structure of $u_{d}(t)$. Based on the parameterized error equation (8.2.11), we propose to use the adaptive controller

$$
\begin{aligned}
u_{d} & =\left[\phi_{\theta 1}^{T}(t) \omega_{\theta 1}(t), \ldots, \phi_{\theta M-1}^{T}(t) \omega_{\theta M-1}(t), 0\right]^{T}+\Phi_{4}^{T}(t) \omega_{4}(t) \\
& +\Phi_{40}(t) \omega_{40}(t)+\Phi_{2}^{T}(t) \omega_{2}(t)+\Phi_{20}(t) y(t)+\Phi_{3}(t) r(t),
\end{aligned}
$$

where $\phi_{\theta i}(t), i=1, \ldots, M-1, \Phi_{j}(t), j=2,20,3,4,40$, are the estimates of $\phi_{\theta i}^{*}$, $i=1, \ldots, M-1, \Phi_{j}^{*}, j=2,20,3,4,40$, to be updated from adaptive laws.

\subsubsection{Parameter Adaption Scheme}

Applying the controller $u_{d}(t)$ as in (8.2.12) to (8.2.11), we obtain

$$
\begin{aligned}
e(t)= & W_{m}(s) L D^{*}\left[\tilde{\Theta}_{N}^{T} \omega_{N}+\left[\tilde{\phi}_{\theta 1}^{T} \omega_{\theta 1}, \ldots, \tilde{\phi}_{\theta M-1}^{T} \omega_{\theta M-1}, 0\right]^{T}\right. \\
& \left.+\tilde{\Phi}_{4}^{T} \omega_{4}+\tilde{\Phi}_{40} \omega_{40}+\tilde{\Phi}_{2}^{T} \omega_{2}+\tilde{\Phi}_{20} y+\tilde{\Phi}_{3} r+d_{N f}\right](t),
\end{aligned}
$$

in which $d_{N f}(t)$ represents the error caused by the unparameterizable uncertainties of the actuator nonlinearity $N(\cdot)$, and all other parts are in terms of their corresponding 
parameter errors, in a desired linear parametrization form. Introducing $\Theta_{0}^{*}=L^{-1}-$ $I=\left\{\theta_{i j}^{*}\right\}$ with $\theta_{i j}^{*}=0$ for $i=1, \ldots, M$ and $j \geq i$, we express (8.2.13) as

$$
\begin{aligned}
& \xi_{m}(s)[e](t)+\Theta_{0}^{*} \xi_{m}(s)[e](t) \\
= & D^{*}\left[\begin{array}{c}
\left(\phi_{1}(t)-\phi_{1}^{*}\right)^{T} \chi_{1}(t) \\
\vdots \\
\left(\phi_{M}(t)-\phi_{M}^{*}\right)^{T} \chi_{M}(t)
\end{array}\right]+D^{*} d_{N f}(t),
\end{aligned}
$$

where, for $\omega(t)=\left[\omega_{4}^{T}(t), \omega_{40}^{T}(t), \omega_{2}^{T}(t), y^{T}(t), r^{T}(t)\right]^{T}, \chi_{1}(t)=\left[\omega_{N 1}^{T}(t), \omega_{\theta 1}^{T}(t), \omega^{T}(t)\right]^{T}$, $\chi_{2}(t)=\left[\omega_{N 2}^{T}(t), \omega_{\theta 2}^{T}(t), \omega^{T}(t)\right]^{T}, \ldots, \chi_{M}(t)=\left[\omega_{N M}^{T}(t), \omega^{T}(t)\right]^{T}$, and $\left(\phi_{i}(t)-\phi_{i}^{*}\right)^{T}$, $i=1,2 \ldots, M$, are the (row) parameter error vectors corresponding to $\chi_{i}(t)$, that is,

$$
\begin{aligned}
\phi_{1}(t) & =\left[\theta_{N 1}^{T}, \phi_{\theta 1}^{T},\left[\Phi_{4}^{T}, \Phi_{40}, \Phi_{2}^{T}, \Phi_{20}, \Phi_{3}\right]_{1}\right]^{T}, \\
\phi_{2}(t) & =\left[\theta_{N 2}^{T}, \phi_{\theta 2}^{T},\left[\Phi_{4}^{T}, \Phi_{40}, \Phi_{2}^{T}, \Phi_{20}, \Phi_{3}\right]_{2}\right]^{T}, \\
& \vdots \\
\phi_{M}(t) & =\left[\theta_{N M}^{T},\left[\Phi_{4}^{T}, \Phi_{2}^{T}, \Phi_{20}, \Phi_{3}\right]_{M}\right]^{T},
\end{aligned}
$$

where $\left[\Phi_{4}^{T}(t), \Phi_{40}(t), \Phi_{2}^{T}(t), \Phi_{20}(t), \Phi_{3}(t)\right]_{i}$ is the $i$ th row of $\left[\Phi_{4}^{T}, \Phi_{40}(t), \Phi_{2}^{T}, \Phi_{20}, \Phi_{3}\right]$.

Consider the modified interactor matrix $\xi_{m}(s)$ given in (2.3.1). For $i=1,2, \ldots, M$, choose $f_{i}(s)$ (with $f_{1}(s)=d_{1}(s)$ ) as a stable polynomial whose degree is equal to the maximum of the degrees of the polynomials $d_{j}(s)$ and $h_{k l}^{m}(s), j=1,2, \ldots, i$, $k=2, \ldots, i, l=1, \ldots, k-1$, and contains $d_{i}(s)$ as a factor, introduce the filters $h_{i}(s)=\frac{1}{f_{i}(s)}, i=1,2, \ldots, M$, and $H(s)=\operatorname{diag}\left\{h_{1}(s), \ldots, h_{M}(s)\right\}$, and define

$$
\bar{e}(t)=H(s) \xi_{m}(s)\left[y-y_{m}\right](t)=\left[\bar{e}_{1}(t), \ldots, \bar{e}_{M}(t)\right]^{T}
$$

for $y(t)=\left[y_{1}(t), \ldots, y_{M}(t)\right]^{T}$ and $y_{m}(t)=\left[y_{m 1}(t), \ldots, y_{m M}(t)\right]^{T}$. For a discrete-time design, the polynomials $f_{i}(s)$ can be simply chosen as $f_{i}(s)=D^{n_{i}}$ for a specified degree $n_{i}, i=1,2, \ldots, M$. 
Defining the auxiliary error signals

$$
\begin{aligned}
e_{\xi}(t) & =\xi_{m}(s)\left[y-y_{m}\right](t)=\left[e_{\xi 1}(t), \ldots, e_{\xi M}(t)\right]^{T} \\
e_{\theta i}(t) & =\left[e_{\xi 1}(t), \ldots, e_{\xi i-1}(t)\right]^{T}, i=2,3, \ldots, M \\
\eta_{i}(t) & =h_{i}(s)\left[e_{\theta i}\right](t) \in R^{i-1}, i=2,3, \ldots, M,
\end{aligned}
$$

operating both sides of (8.2.14) by $H(s)$, with $\bar{d}_{N}=H(s)\left[D^{*} d_{N f}\right](t)=\left[\bar{d}_{N 1}, \ldots, \bar{d}_{N M}\right]^{T}$, we have

$$
\begin{aligned}
\bar{e}_{1} & =d_{1}^{*} h_{1}(s)\left[\left(\phi_{1}-\phi_{1}^{*}\right)^{T} \chi_{1}\right](t)+\bar{d}_{N 1}, \\
\bar{e}_{2}+\theta_{2}^{*} \eta_{2}= & d_{2}^{*} h_{2}(s)\left[\left(\phi_{2}-\phi_{2}^{*}\right)^{T} \chi_{2}\right](t)+\bar{d}_{N 2}, \\
& \vdots \\
\bar{e}_{M}+\theta_{M}^{* T} \eta_{M} & =d_{M}^{*} h_{M}(s)\left[\left(\phi_{M}-\phi_{M}^{*}\right)^{T} \chi_{M}\right](t)+\bar{d}_{N M},
\end{aligned}
$$

where $\theta_{i}^{*}=\left[\theta_{i 1}^{*}, \ldots, \theta_{i i-1}^{*}\right]^{T}$, for $i=2, \ldots, M$. Introduce the auxiliary signals $\zeta_{i}(t)=$ $h_{i}(s)\left[\chi_{i}\right](t)$ and $\xi_{i}(t)=\phi_{i}^{T}(t) \zeta_{i}(t)-h_{i}(s)\left[\phi_{i}^{T} \chi_{i}\right](t)$, let $d_{i}(t)$ be the estimate of $d_{i}^{*}, i=$ $1, \ldots, M, \theta_{i}(t)$ be the estimate of $\theta_{i}^{*}, i=2, \ldots, M$, and define the estimation errors

$$
\begin{aligned}
\epsilon_{1}(t)= & \bar{e}_{1}(t)+d_{1}(t) \xi_{1}(t) \\
\epsilon_{2}(t)= & \bar{e}_{2}(t)+\theta_{2}(t) \eta_{2}(t)+d_{2}(t) \xi_{2}(t) \\
& \vdots \\
\epsilon_{M}(t)= & \bar{e}_{M}(t)+\theta_{M}^{T}(t) \eta_{M}(t)+d_{M}(t) \xi_{M}(t)
\end{aligned}
$$

Then, from (8.2.20)-(8.2.21), we have the error model

$$
\begin{aligned}
\epsilon_{1} & =d_{1}^{*}\left(\phi_{1}-\phi_{1}^{*}\right)^{T} \zeta_{1}+\left(d_{1}-d_{1}^{*}\right) \xi_{1}+\bar{d}_{N 1}, \\
\epsilon_{2} & =\left(\theta_{2}-\theta_{2}^{*}\right)^{T} \eta_{2}+d_{2}^{*}\left(\phi_{2}-\phi_{2}^{*}\right)^{T} \zeta_{2}+\left(d_{2}-d_{2}^{*}\right) \xi_{2}+\bar{d}_{N 2}, \\
& \vdots \\
\epsilon_{M} & =\left(\theta_{M}-\theta_{M}^{*}\right)^{T} \eta_{M}+d_{M}^{*}\left(\phi_{M}-\phi_{M}^{*}\right)^{T} \zeta_{M}+\left(d_{M}-d_{M}^{*}\right) \xi_{M}+\bar{d}_{N M} .
\end{aligned}
$$


In this model, the parameters $d_{i}(t), i=1,2, \ldots, M$, are the estimates (to be updated from some adaptive laws) of $d_{i}^{*}, i=1,2, \ldots, M$, which are the diagonal elements of the matrix $D^{*}$ in the LDU decomposition (2.3.3) of the high frequency gain matrix $K_{p}$ of the system transfer matrix $G(s)$. The signals $\bar{d}_{N i}(t), i=1,2, \ldots, M$, are bounded disturbances due to the unparametrizable uncertainties of the actuator nonlinearity.

Adaptive laws. Based on the error model (8.2.22), we choose the adaptive laws

$$
\begin{aligned}
& \dot{\theta}_{i}(t)=\frac{\Gamma_{\theta i} \epsilon_{i}(t) \eta_{i}(t)}{m^{2}(t)}+f_{\theta i}, i=2, \ldots, M, \\
& \dot{\phi}_{i}(t)=\frac{\operatorname{sign}\left[d_{i}^{*}\right] \Gamma_{\phi i} \epsilon_{i} \zeta_{i}}{m^{2}(t)}+f_{\phi i}, i=1, \ldots, M, \\
& \dot{d}_{i}(t)=\frac{\gamma_{i} \epsilon_{i}(t) \xi_{i}(t)}{m^{2}(t)}+f_{d i}, i=1, \ldots, M,
\end{aligned}
$$

where $m(t)=\left(1+\sum_{i=1}^{M} \zeta_{i}^{T} \zeta_{i}+\sum_{i=1}^{M} \xi_{i}^{2}+\sum_{i=2}^{M} \eta_{i}^{T} \eta_{i}\right)^{1 / 2}$, and $\Gamma_{\theta i}=\Gamma_{\theta i}^{T}>0, i=$ $2,3, \ldots, M, \Gamma_{\phi i}=\Gamma_{\phi i}^{T}>0, i=1,2, \ldots, M$, and $\gamma_{i}>0, i=1,2, \ldots, M$. The functions $f_{\theta i}(t), f_{\phi i}(t)$ and $f_{d i}(t), i=1,2, \ldots, M$, are robustifying design signals [44], for robustness of the adaptive laws with respect to the bounded disturbances $\bar{d}_{N i}(t)$, $i=1,2, \ldots, M$.

It is crucial for the parameter estimates $\theta_{N i}(t)$ of the actuator nonlinearity parameters $\theta_{N i}^{*}$ to stay in their required intervals for the implementation of their desired (nonsingular, that is, no division by zero) adaptive inverses whose parameters have certain bounds to reflect some physical meaning.

The combined parameter projection and switching- $\sigma$ modification. A switching- $\sigma$ modification [42] $f(t)$ for the estimate $\psi(t)$ of a parameter vector $\psi^{*}$ has the desired properties: $\tilde{\psi}^{T}(t) \Gamma^{-1} f(t) \leq 0$ and and $\lim _{\|\psi(t)\|_{2} \rightarrow \infty} \tilde{\psi}^{T}(t) \Gamma^{-1} f(t)=\infty$, which are crucial for stability of the adaptive laws. A parameter projection $f(t)[84]$ satisfies: $\tilde{\psi}^{T}(t) \Gamma^{-1} f(t) \leq 0$ too, and also ensure the components of $\psi(t)$ stay in the bounds of $\psi^{*}$. 
A parameter projection design $f(t)$ is applied to the portion $f_{N i}(t)$ of $f_{\phi i}(t)=$ $\left[f_{N i}^{T}(t), \bar{f}_{\phi i}^{T}(t)\right]^{T}$ in (8.2.24), corresponding to $\theta_{N i}(t)$ in $\phi_{i}(t)=\triangleq\left[\theta_{N i}^{T}(t), \bar{\phi}_{i}(t)\right]^{T}$ in (8.2.15), to ensure that the components of $\theta_{N i}(t)$ stay in their desired intervals.

To be combined with parameter projection, a switching- $\sigma$ modification $f(t)$ is used to $f_{\theta i}(t)$ for $\theta_{i}$ in (8.2.23), to $\bar{f}_{\phi i}(t)$ in $f_{\phi i}(t)=\left[f_{N i}^{T}(t), \bar{f}_{\phi i}^{T}(t)\right]^{T}$ for $\bar{\phi}_{i}(t)$ of $\phi_{i}(t)=$ $\left[\theta_{N i}^{T}(t), \bar{\phi}^{T}(t)\right]^{T}$ in (8.2.24), and to $f_{d i}(t)$ for $d_{i}(t)$ in (8.2.25).

\subsubsection{Stability}

Consider the positive definite function

$$
V\left(\tilde{\theta}_{i}, \tilde{\phi}_{i}, \tilde{d}_{i}\right)=\sum_{i=2}^{M} \tilde{\theta}_{i}^{T} \Gamma_{\theta i}^{-1} \tilde{\theta}_{i}+\sum_{i=1}^{M}\left|d_{i}^{*}\right| \tilde{\phi}_{i}^{T} \Gamma_{\phi i}^{-1} \tilde{\phi}_{i}+\sum_{i=1}^{M} \gamma_{i}^{-1} \tilde{d}_{i}^{2}
$$

where $\tilde{\theta}_{i}(t), \tilde{\phi}_{i}(t)$ and $\tilde{d}_{i}(t)$ are parameter errors, and its time-derivative of $V$

$$
\dot{V} \leq-\sum_{i=1}^{M} \frac{\epsilon_{i}^{2}(t)}{m^{2}(t)}-\sum_{i=1}^{M} \frac{\left(\bar{d}_{N i}(t)-\epsilon_{i}(t)\right)^{2}}{m^{2}(t)}+\sum_{i=1}^{M} \frac{\bar{d}_{N i}^{2}(t)}{m^{2}(t)} .
$$

It follows that all parameter estimates are bounded (either from parameter projection bounding or from the switching- $\sigma$ property: $\left.\lim _{\|\psi(t)\|_{2} \rightarrow \infty} \tilde{\psi}^{T}(t) \Gamma^{-1} f(t)=\infty\right)$. From the last inequality, it follows that

$$
\int_{t_{1}}^{t_{2}} \sum_{i=1}^{M} \frac{\epsilon_{i}^{2}(t)}{m^{2}(t)} d t \leq a_{1}+b_{1} \int_{t_{1}}^{t_{2}} \sum_{i=1}^{M} \frac{\bar{d}_{N i}^{2}(t)}{m^{2}(t)} d t
$$

for some constants $a_{1}>0, b_{1}>0$, and any $t_{2}>t_{1}>0$. From (8.2.26) and with $f_{\theta i}, f_{\phi i}$ and $f_{d i}$ in (8.2.23)-(8.2.25), we can establish:

Lemma 8.2.1. The adaptive laws (8.2.23)-(8.2.25) ensure that all parameter estimates are bounded, all components of $\theta_{N i}(t)$ are within desired regions, and $\frac{\epsilon_{i}^{2}(t)}{m^{2}(t)}$, $\left\|\dot{\theta}_{i}(t)\right\|_{2}^{2},\left\|\dot{\phi}_{i}(t)\right\|_{2}^{2}$, and $\dot{d}_{i}^{2}(t)$, are bounded by $\frac{\vec{d}_{N i}^{2}(t)}{m^{2}(t)}$ in the mean sense (8.2.26).

With these desired adaptive law properties, the closed-loop system is well-defined (based on the boundedness of adaptive parameter estimates), a feedback structure 
can be derived whose loop gain is proportional to $\frac{\epsilon_{i}(t)}{m(t)}, \dot{\theta}_{i}(t), \dot{\phi}_{i}(t)$ and $\dot{d}_{i}(t)$, and a small-gain analysis can be carried out to establish the desired closed-loop control system properties:

Theorem 8.2.1. The adaptive control scheme consisting of an adaptive actuator nonlinearity inverse (8.1.6) and an adaptive output feedback control law (8.2.12) updated from the adaptive laws (8.2.23)-(8.2.25), when applied to the system (8.1.1) with some specific actuator nonlinearities $u(t)=N(v(t))$, ensures that all closed-loop system signals are bounded and the tracking error $e(t)=y(t)-y_{m}(t)$ also satisfies (8.2.26) similarly.

Both switching- $\sigma$ and parameter projection modifications have the desired feature that they preserve the asymptotic output tracking property when the system disturbances and unmodeled dynamics disappear from the controlled system. Therefore, we also have

Corollary 8.2.1. If, in addition, the actuator nonlinearity parametrization error $d_{N}(t)$ disappears after $t \geq T_{0}$ for some finite $T_{0}>0$, or $d_{N}(t) \in L^{2}$, then the tracking error $e(t)=y(t)-y_{m}(t)$ has the desired properties: $e(t) \in L^{2}$ and $\lim _{t \rightarrow \infty} e(t)=0$.

In next section, we will study a MIMO System application: adaptive control of aircraft flight control systems with synthetic jet actuators whose nonlinear characteristics are to be compensated by adaptive inverses, and present simulation results to show the desired performance of adaptive actuator nonlinearity compensation. 


\subsection{Aircraft Flight Control Simulation Study}

The system we apply our adaptive control design to is a lateral-directional of the Innovative Control Effector (ICE) aircraft [75] with synthetic jet actuators:

$$
\dot{x}(t)=A x(t)+B u(t), \quad y(t)=C x(t),
$$

where the state vector signal is $x(t)=\left[v_{b}(t), p_{b}(t), r_{b}(t), \phi(t)\right]^{T}$ with $v_{b}(t)$ being the body-axis lateral-directional velocity whose unit is $\mathrm{ft} / \mathrm{sec}, p_{b}(t)$ and $r_{b}(t)$ being the roll rate and yaw rate whose units are $\mathrm{rad} / \mathrm{sec}$, and $\phi(t)$ being the roll angle whose unit is rad, the control input signal is $u(t)=\left[u_{1}(t), u_{2}(t)\right]^{T}$ with $u_{1}(t)$ and $u_{2}(t)$ being the virtual deflections generated by upper leading-edge and wing-tip synthetic jet actuators whose units are degree, and the output vector signal is chosen as $y(t)=$ $\left[v_{b}(t), \phi(t)\right]^{T}$. As described in Section 2.2, the nonlinearity of the synthetic jet actuator $u_{i}(t)=N\left(v_{i}(t)\right)$ can be characterized as $u_{i}(t)=N_{i}\left(\theta_{i}^{*} ; v_{i}(t)\right)=\theta_{i 2}^{*}-\frac{\theta_{i 1}^{*}}{v_{i}(t)}$, for $i=1,2$.

From the structure knowledge of the parameters $A, B$, and $C$, we can obtain the interactor matrix as $\xi_{m}(s)=\operatorname{diag}\left\{(s+1)^{2},(s+1)^{2}\right\}$ and the signs of leading principal minors of $K_{p}$ as $\operatorname{sign}\left(\Delta_{1}\right)=1, \operatorname{sign}\left(\Delta_{2}\right)=-1$. Hence, the reference system is chosen as $W_{m}(s)=\xi_{m}^{-1}(s)=\operatorname{diag}\left\{1 /(s+1)^{2}, 1 /(s+1)^{2}\right\}$. We then apply the adaptive output feedback inverse compensation control law $u_{d}(t)$ as in (8.2.12) to generate the control input $v(t)=\widehat{N I}\left(u_{d}(t)\right)$ as in (8.1.14), to make the plant output $y(t)$ track the reference output $y_{m}(t)=W_{m}(s)[r](t)$.

Simulation Results For the simulation (i): constant reference input $r(t)=$ $\left[6(\mathrm{ft} / \mathrm{s}), \frac{8 \pi}{180}(\mathrm{rad})\right]^{T}$, the response of output signal $y(t)$, commanded input signal $v(t)$, plant input signal $u(t)$, and feedback control signal $u_{d}(t)$ is shown in Fig. 8.1Fig. 8.3. From Fig. 8.1, we can see that the output signal $y(t)$ tracks the reference signal $y_{m}(t)$. Since the parameters of the adaptive inverse $\widehat{N I}\left(u_{d}(t)\right)$ may 
not converge to the nominal values, the plant input signal $u(t)$ may not follow the feedback control signal $u_{d}(t)$, as illustrated in Fig. 8.3. The effectiveness of the developed adaptive actuator nonlinearity compensation scheme is further verified by the simulation (ii) as shown in Fig. 8.4-Fig. 8.6, where the reference input is $r(t)=\left[10+4 \sin \left(\frac{\pi}{50} t\right)(\mathrm{ft} / \mathrm{s}), \frac{8 \pi}{180} \sin \left(\frac{\pi}{55} t\right)(\mathrm{rad})\right]^{T}$.
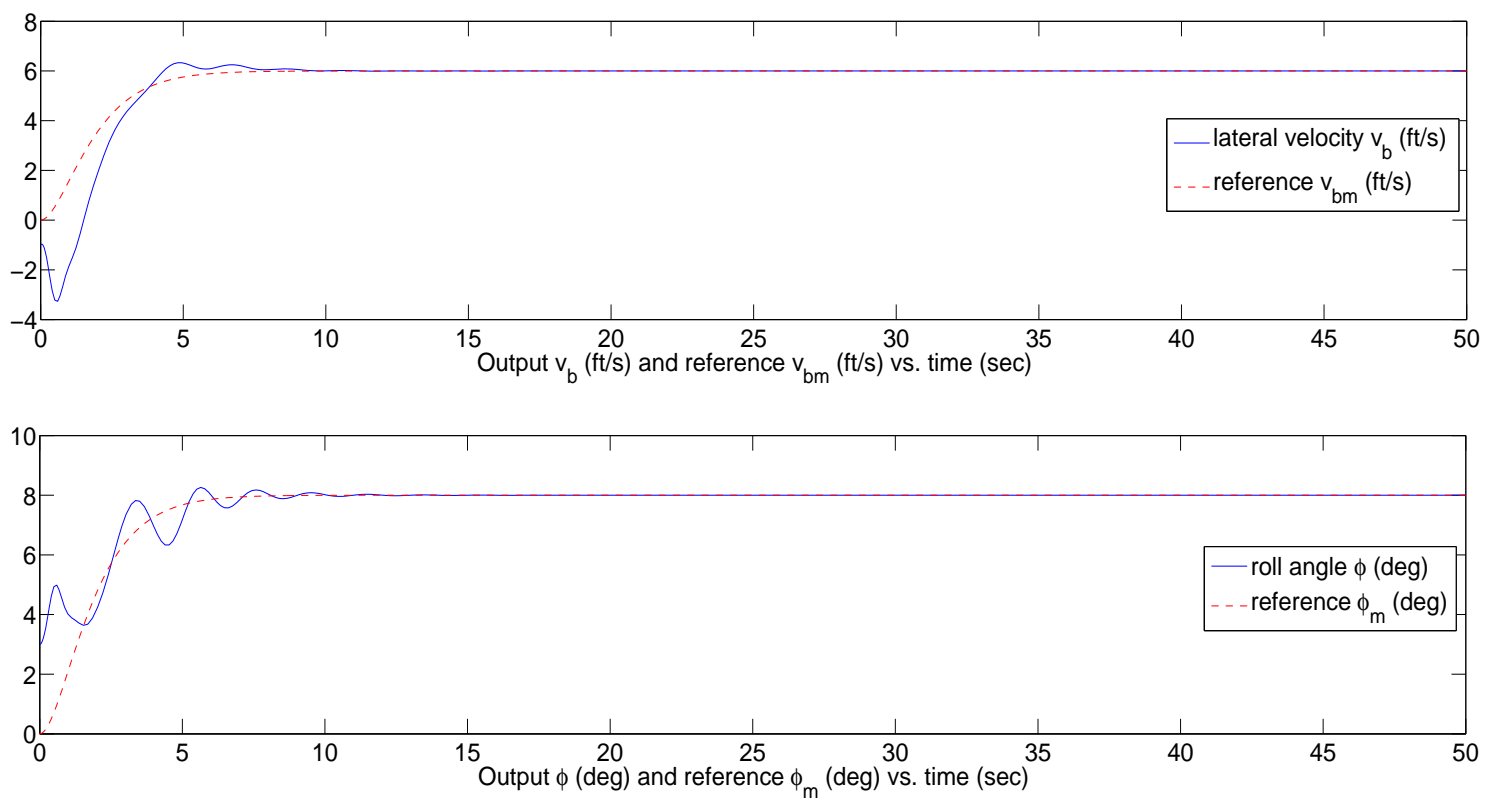

Figure 8.1: Aircraft outputs (solid) vs. reference outputs (dotted) (case i)

\section{Summary}

Adaptive compensation of uncertain actuator nonlinearities in multi-input multioutput systems has certain unique technical issues caused by bilinear parameters resulted from the actuator nonlinearities and system dynamics. In this chapter we have solved some such key issues with output feedback design, using a new controller parametrization to deal with the uncertain parameters of the system high frequency gain matrix and the special structure of the actuator nonlinearity parametrization. 

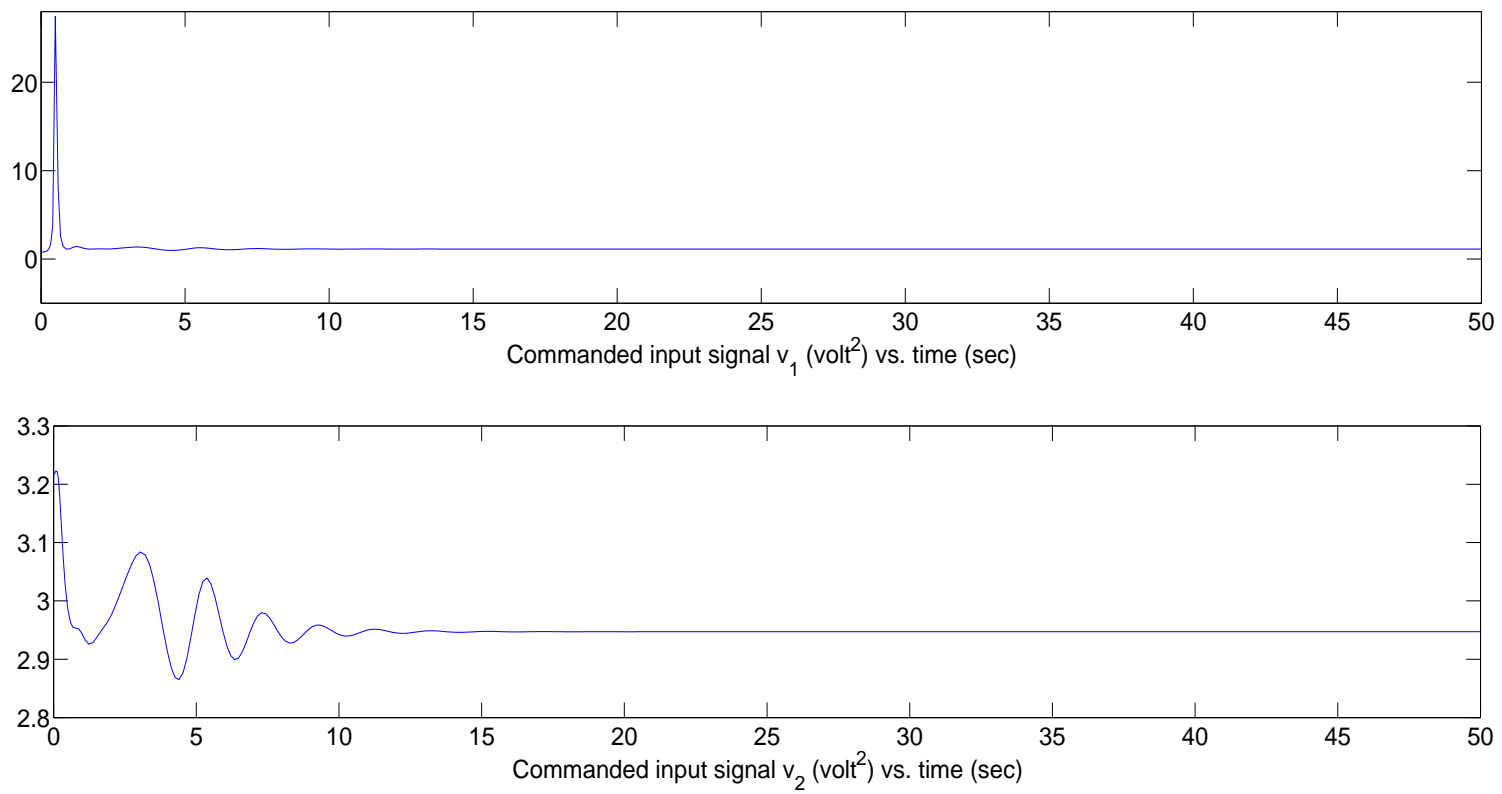

Figure 8.2: Commanded control input signal $v(t)$ (case i)
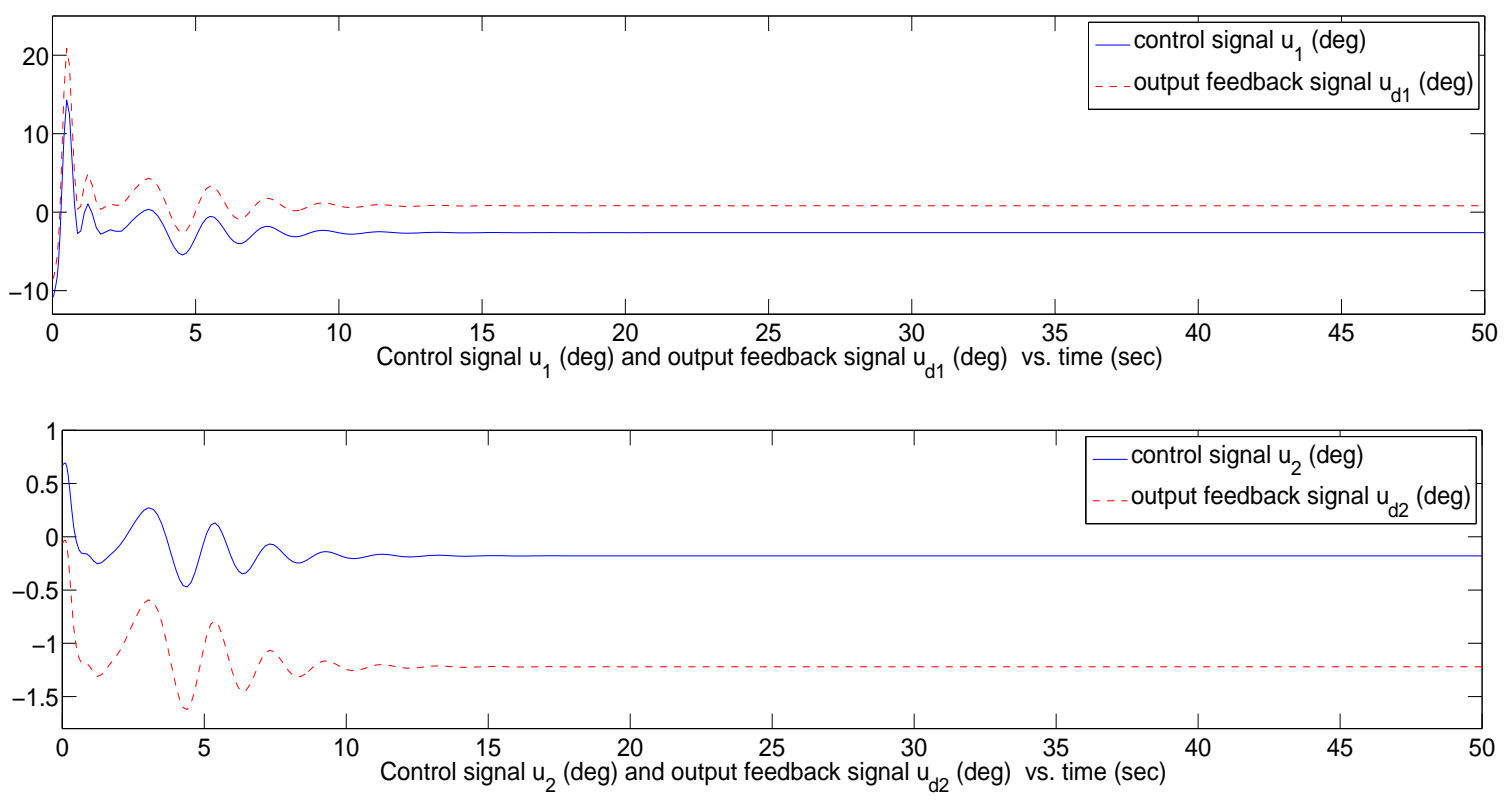

Figure 8.3: Plant input signal $u(t)$ and feedback control signal $u_{d}(t)$ (case i) 

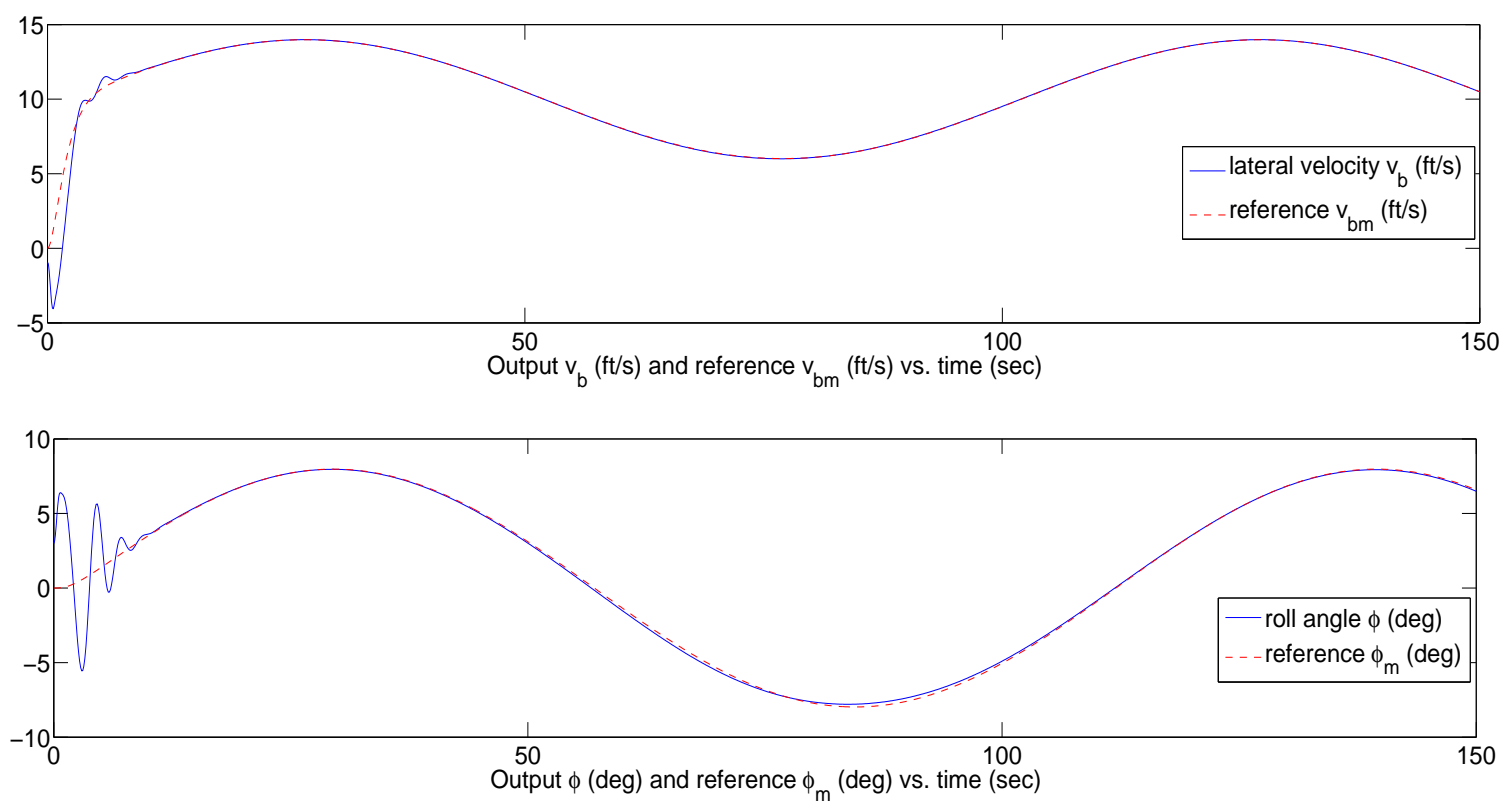

Figure 8.4: Aircraft outputs (solid) vs. reference outputs (dotted) (case ii)
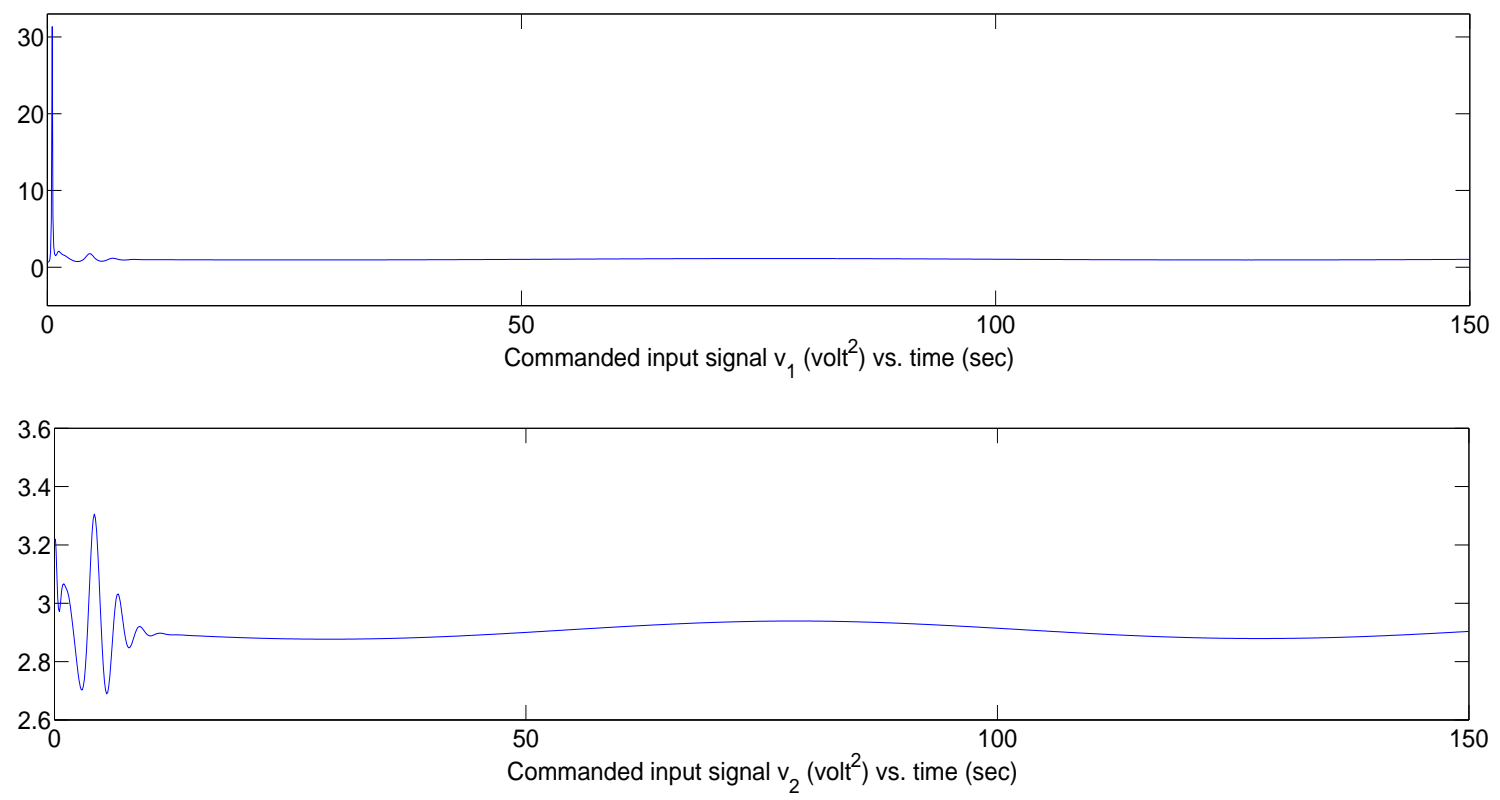

Figure 8.5: Commanded control input signal $v(t)$ (case ii) 

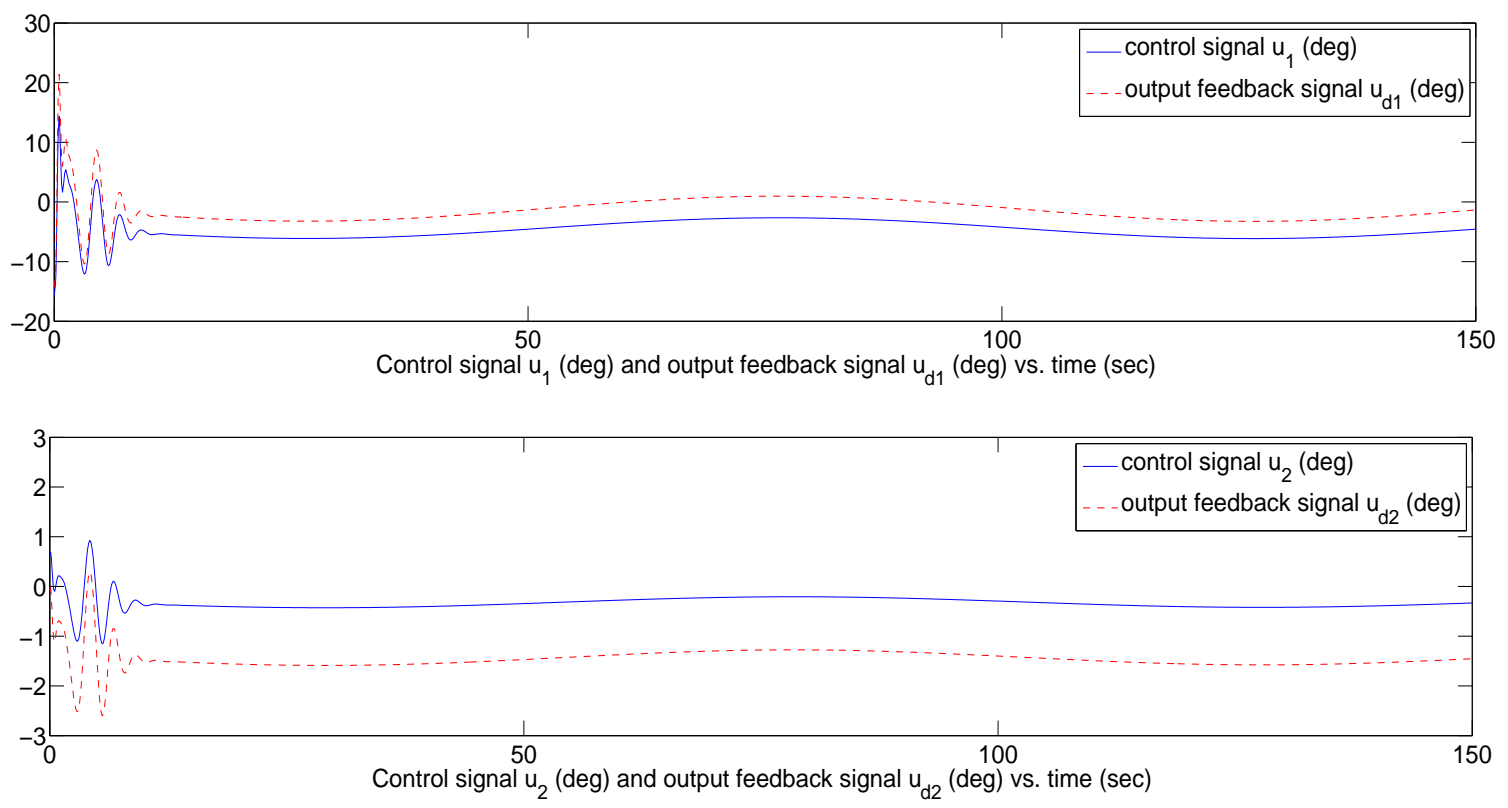

Figure 8.6: Plant input signal $u(t)$ and feedback control signal $u_{d}(t)$ (case ii)

Such a new controller structure is based on the LDU decomposition of the high frequency gain matrix, leading to a linear parametrization of the error system. An expanded estimation error signal is introduced for stable adaptive laws updating the controller and inverse parameters. A combined switching- $\sigma$ and parameter projection modification is employed for parameter adaptation for both the robustness with respect to actuator nonlinearity parametrization errors and the nonsingularity of the adaptive inverse design. Such an adaptive compensation scheme has desired system stability and tracking properties as stated in the chapter and verified by simulation results from an aircraft flight control application example in which synthetic jet actuator nonlinearities are compensated by their adaptive inverses. 


\section{Chapter 9}

\section{Adaptive State Feedback Disturbance Rejection for MIMO Linear Time-Invariant Systems}

In this chapter, we will study the adaptive disturbance rejection problem for MIMO linear time-invariant (LTI) systems by using state feedback control. A nominal state feedback control law with a parameterizable signal for disturbance cancelation will be derived. Then, the state feedback adaptive control scheme will be developed based on the nominal state feedback controller for LTI systems with unknown system parameters and uncertain disturbances.

\subsection{Problem Statement}

Consider a MIMO LTI system with uncertain disturbances:

$$
\begin{aligned}
\dot{x}(t) & =A x(t)+B u(t)+B_{d} d(t), \\
y(t) & =C x(t),
\end{aligned}
$$

where $x(t) \in R^{n}, y(t) \in R^{M}$, and $u(t) \in R^{M}$ are state, output, and control input vector signals, $d(t) \in R^{q}$ represents the uncertain actuation disturbances, and $A \in$ $R^{n \times n}, B \in R^{n \times M}, C \in R^{M \times n}$, and $B_{d} \in R^{n \times q}$ are some unknown constant matrices. 
For the study of adaptive disturbance rejection, elements of the disturbance signal $d(t)=\left[d_{1}(t), d_{2}(t), \ldots, d_{q}(t)\right]^{T}$ are characterized as

$$
d_{j}(t)=\bar{d}_{j 0}+\sum_{k=1}^{p_{j}} \bar{d}_{j k} \omega_{j k}(t),
$$

with unknown constants $\bar{d}_{j 0}, \bar{d}_{j k}$ and known signals $\omega_{j k}(t), j=1,2, \ldots, q, k=$ $1,2, \ldots, p_{j}$

The objective is to design a state feedback control law $u(t)$ to cancel the effect of the disturbance $d(t)$ on the output signal $y(t)$ and make the output signal $y(t)$ track a given reference signal $y_{m}(t)$ :

$$
y_{m}(t)=W_{m}(s)[r](t), \quad W_{m}(s)=\xi_{m}^{-1}(s),
$$

where $r(t)$ is a bounded reference input signal.

To proceed the adaptive control design, we make the following assumptions: (A1) the system input-output transfer matrix $G_{0}(s)=C(s I-A)^{-1} B$ has full rank, all zeros of $G_{0}(s)$ have negative real parts; (A2) the modified left interactor matrix $\xi_{m}(s)$ of $G_{0}(s)$ is known; (A3) all leading principal minors $\Delta_{i}, i=1,2, \ldots, M$ of the high frequency gain matrix $K_{p}$ of $G_{0}(s)$ are nonzero and their signs are known; (A4) $(A, B)$ is controllable and $(A, C)$ is observable; (A5) the transfer matrix $Z_{l}^{-1}(s) Z_{d}(s)$ is proper, for the fractional descriptions of the input-output transfer matrix $G_{0}(s)=$ $C(s I-A)^{-1} B$ and the disturbance-output transfer matrix $G_{d}(s)=C(s I-A)^{-1} B_{d}$ :

$$
G_{0}(s)=P_{l}^{-1}(s) Z_{l}(s), \quad G_{d}(s)=P_{l}^{-1}(s) Z_{d}(s)
$$

where $P_{l}(s), Z_{l}(s)$, and $Z_{d}(s)$ are polynomial matrices, and $Z_{l}(s)$ and $P_{l}(s)$ are left coprime with row degrees of $P_{l}(s)$ satisfying $\operatorname{deg}_{r_{i}}\left[P_{l}(s)\right] \leq \nu, i=1,2, \ldots, M$, where $\nu$ is the observability index of $(A, C)$. 


\subsection{Nominal State Feedback Control Design}

Before developing the adaptive state feedback control scheme for the system (9.1.1) with unknown parameters and uncertain disturbances, we need to design a nominal state feedback controller with a parameterizable form to cancel the disturbances and achieve exact plant-model matching by assuming that the system parameters and disturbance parameters are known.

We choose the nominal state feedback controller as

$$
u(t)=K_{1}^{* T} x(t)+K_{2}^{*} r(t)+k_{3}^{*}(t)
$$

where $k_{3}^{*}(t) \in R^{M}$ is used to cancel the effect of the disturbance $B_{d} d(t)$ on tracking error $e(t)=y(t)-y_{m}(t)$, and constant parameters $K_{1}^{*} \in R^{n \times M}$ and $K_{2}^{*} \in{ }^{M \times M}$ satisfy the matching equation (2.4.3):

$$
C\left(s I-A-B K_{1}^{* T}\right)^{-1} B K_{2}^{*}=W_{m}(s), K_{2}^{*-1}=K_{p}
$$

The existence of $K_{1}^{*}$ and $K_{2}^{*}$ of the matching equation (9.2.2) has been shown in Section 2.4.1. Applying the controller (9.2.1) to the system (9.1.1), we obtain the closed-loop system as

$$
\begin{aligned}
y(t)= & C\left(s I-A-B K_{1}^{* T}\right)^{-1} B K_{2}^{*}[r](t)+C\left(s I-A-B K_{1}^{* T}\right)^{-1} B\left[k_{3}^{*}\right](t) \\
& +C\left(s I-A-B K_{1}^{* T}\right)^{-1} B_{d}[d](t)+C e^{\left(A+B K_{1}^{* T}\right) t} x(0) .
\end{aligned}
$$

From the reference system (9.1.3) and the matching equation (9.2.2), we have the output tracking error $e(t)=y(t)-y_{m}(t)$ as

$$
e(t)=C\left(s I-A-B K_{1}^{* T}\right)^{-1} B\left[k_{3}^{*}\right]+C\left(s I-A-B K_{1}^{* T}\right)^{-1} B_{d}[d]+C e^{\left(A+B K_{1}^{* T}\right) t} x(0) .
$$

Remark 9.2.1. From the error equation (9.2.4), we may choose to set $k_{3}^{*}(t)$ as

$$
k_{3}^{*}(t)=K_{2}^{*} \xi_{m}(s) C\left(s I-A-B K_{1}^{* T}\right)^{-1} B_{d}[d](t)
$$


to make $e(t)=C e^{\left(A+B K_{1}^{* T}\right) t} x(0)$, i.e., $\lim _{t \rightarrow \infty} e(t)=0$. However, such a choice of $k_{3}^{*}(t)$ needs to be parameterized as $k_{3}^{*}(t)=\frac{P_{a}(s)}{\Lambda(s)}$ with a known stable polynomial $\Lambda(s)$ and an unknown polynomial matrix $P_{a}(s)$, such that $\frac{P_{a}(s)}{\Lambda(s)}$ is proper (such a parametrization is crucial for the adaptive control design). Therefore, the direct choice of $k_{3}^{*}(t)$ as (9.2.5) may not be suitable for the adaptive control design. In the following, we will derive a parameterizable $k_{3}^{*}(t)$, which can cancel the effect of the disturbances:

$$
C\left(s I-A-B K_{1}^{* T}\right)^{-1} B\left[k_{3}^{*}\right](t)=-C\left(s I-A-B K_{1}^{* T}\right)^{-1} B_{d}[d](t),
$$

so that $\lim _{t \rightarrow \infty} e(t)=0$.

In order to obtain a parameterizable $k_{3}^{*}(t)$ to cancel the disturbances, we first introduce a matching equation as shown in the following lemma.

Lemma 9.2.1. Given $K_{1}^{*}$ and $K_{2}^{*}$ satisfying the matching equation (9.2.2), the following plant-model matching equation holds:

$Z_{l}^{-1}(s) P_{l}(s)-K_{1}^{* T}(s I-A+L C)^{-1} B Z_{l}^{-1}(s) P_{l}(s)-K_{1}^{* T}(s I-A+L C)^{-1} L=K_{2}^{*} \xi_{m}(s)$,

where $L$ is such that $\operatorname{det}(s I-A+L C)=\Lambda(s)$ being a chosen stable polynomial.

Proof: Consider the system (9.1.1) without the disturbances by using the state observer representation:

$$
\begin{aligned}
\dot{x}(t) & =(A-L C) x(t)+B u(t)+L y(t), \\
y(t) & =C x(t) .
\end{aligned}
$$

Since $(A, C)$ is observable, there exists a matrix $L \in R^{n \times M}$, such that $\operatorname{det}(s I-A+$ $L C)=\Lambda(s)$ for a chosen stable polynomial. Then, the state feedback controller can be parameterized as

$$
\begin{aligned}
u(t) & =K_{1}^{* T} x(t)+K_{2}^{*} r(t) \\
& =K_{1}^{* T}(s I-A+L C)^{-1} B[u]+K_{1}^{* T}(s I-A+L C)^{-1} L[y]+K_{2}^{*} r
\end{aligned}
$$


For the open-loop system, we have

$$
u(t)=Z_{l}^{-1}(s) P_{l}(s)[y](t)
$$

with $Z_{l}(s)$ and $P_{l}(s)$ being left coprime and $\operatorname{deg}_{r_{i}}\left[P_{l}(s)\right] \leq \nu, i=1,2, \ldots, M$, as defined in (9.1.4). Substituting (9.2.10) in (9.2.9), we have

$$
\left(Z_{l}^{-1}(s) P_{l}(s)-K_{1}^{* T}(s I-A+L C)^{-1} B Z_{l}^{-1}(s) P_{l}(s)-K_{1}^{* T}(s I-A+L C)^{-1} L\right)[y]=K_{2}^{*} r .
$$

Substituting $u(t)=K_{1}^{* T} x(t)+K_{2}^{*} r(t)$ in the system (9.2.8), we obtain another closedloop system expression:

$$
y(t)=C\left(s I-A-B K_{1}^{* T}\right)^{-1} B K_{2}^{*}[r](t) .
$$

From the matching equation $(9.2 .2)$, there exist constant parameters $K_{1}^{*}$ and $K_{2}^{*}$ to make the closed-loop system (9.2.12) as

$$
y(t)=W_{m}(s)[r](t)
$$

Since the closed-loop systems (9.2.11) and (9.2.13) are equivalent, we obtain

$$
Z_{l}^{-1}(s) P_{l}(s)-K_{1}^{* T}(s I-A+L C)^{-1} B Z_{l}^{-1}(s) P_{l}(s)-K_{1}^{* T}(s I-A+L C)^{-1} L=K_{2}^{*} \xi_{m}(s) .
$$

Therefore, the constant parameters $K_{1}^{*}$ and $K_{2}^{*}$, which satisfy the matching equation (9.2.2), can also make the matching equation (9.2.7) hold.

In the following Lemma 9.2.2 and Lemma 9.2.3, we will show that there exists a parameterizable $k_{3}^{*}(t)$ such that the nominal controller (9.2.1) can reject the disturbance $d(t)$ and make the output signal $y(t)$ track the reference signal $y_{m}(t)$. 
Lemma 9.2.2. The state feedback controller (9.2.1) with $K_{1}^{*}$ and $K_{2}^{*}$ satisfying the matching equation (9.2.2) and

$$
\begin{aligned}
k_{3}^{*}(t)= & -\left(Z_{l}^{-1}(s) Z_{d}(s)-K_{1}^{* T}(s I-A+L C)^{-1} B Z_{l}^{-1}(s) Z_{d}(s)\right. \\
& \left.+K_{1}^{* T}(s I-A+L C)^{-1} B_{d}\right)[d](t),
\end{aligned}
$$

for $\operatorname{det}(s I-A+L C)$ being a chosen stable polynomial, ensures $\lim _{t \rightarrow \infty}\left(y(t)-y_{m}(s)\right)=$ 0 exponentially.

Proof: From Lemma 9.2.1, we can parameterize $k_{3}^{*}(t)$ as

$$
\begin{aligned}
k_{3}^{*}(t)= & \left(Z_{l}^{-1}(s) Z_{d}(s)-K_{1}^{* T}(s I-A+L C)^{-1} B Z_{l}^{-1} Z_{d}(s)\right. \\
& \left.+K_{1}^{* T}(s I-A+L C)^{-1} B_{d}\right)[d](t) \\
= & -\left(\left(Z_{l}^{-1}(s) P_{l}(s)-K_{1}^{* T}(s I-A+L C)^{-1} B Z_{l}^{-1}(s) P_{l}(s)-K_{1}^{* T}(s I-A+L C)^{-1} L\right.\right. \\
& \left.\left.+K_{1}^{* T}(s I-A+L C)^{-1} L\right) P_{l}^{-1}(s) Z_{d}(s)+K_{1}^{* T}(s I-A+L C)^{-1} B_{d}\right)[d](t) \\
= & -\left(K_{2}^{*} \xi_{m}(s) P_{l}^{-1}(s) Z_{d}(s)+K_{1}^{* T}(s I-A+L C)^{-1} L P_{l}^{-1}(s) Z_{d}(s)\right. \\
& \left.+K_{1}^{* T}(s I-A+L C)^{-1} B_{d}\right)[d](t) .
\end{aligned}
$$

In view of $(9.2 .16),(9.1 .4)$ (i.e., $\left.P_{l}^{-1}(s) Z_{d}(s)=C(s I-A)^{-1} B_{d}\right)$, and the matching 
equation (9.2.2), we have

$$
\begin{aligned}
& C\left(s I-A-B K_{1}^{* T}\right)^{-1} B\left[k_{3}^{*}\right](t) \\
= & -\left(C(s I-A)^{-1} B_{d}+C\left(s I-A-B K_{1}^{* T}\right)^{-1} B K_{1}^{* T}(s I-A+L C)^{-1} L C(s I-A)^{-1} B_{d}\right. \\
& \left.+C\left(s I-A-B K_{1}^{* T}\right)^{-1} B K_{1}^{* T}(s I-A+L C)^{-1} B_{d}\right)[d](t) \\
= & -C\left(s I-A-B K_{1}^{* T}\right)^{-1}\left(\left(s I-A-B K_{1}^{* T}\right)(s I-A)^{-1}\right. \\
& \left.+B K_{1}^{* T}(s I-A+L C)^{-1} L C(s I-A)^{-1}+B K_{1}^{* T}(s I-A+L C)^{-1}\right) B_{d}[d](t) \\
= & -C\left(s I-A-B K_{1}^{* T}\right)^{-1}\left(I-B K_{1}^{* T}(s I-A)^{-1}\right. \\
& \left.+B K_{1}^{* T}(s I-A+L C)^{-1} L C(s I-A)^{-1}+B K_{1}^{* T}(s I-A+L C)^{-1}\right) B_{d}[d](t) \\
= & -C\left(s I-A-B K_{1}^{* T}\right)^{-1}\left(I-B K_{1}^{* T}(s I-A+L C)^{-1}\left((s I-A+L C)(s I-A)^{-1}\right.\right. \\
& \left.\left.-L C(s I-A)^{-1}-I\right)\right) B_{d}[d](t) .
\end{aligned}
$$

Substituting

$$
\begin{aligned}
& (s I-A+L C)(s I-A)^{-1}-L C(s I-A)^{-1}-I \\
= & I+L C(s I-A)^{-1}-L C(s I-A)^{-1}-I \\
= & 0
\end{aligned}
$$

in $(9.2 .17)$, we have

$$
C\left(s I-A-B K_{1}^{* T}\right)^{-1} B\left[k_{3}^{*}\right](t)=-C\left(s I-A-B K_{1}^{* T}\right)^{-1} B_{d}[d](t) .
$$

Then, from (9.2.4) and (9.2.19), we obtain that the output tracking error converges to zero exponentially: $\lim _{t \rightarrow \infty} e(t)=\lim _{t \rightarrow \infty} C e^{\left(A+B K_{1}^{* T}\right) t} x(0)=0$.

Next, we will show that $k_{3}^{*}(t)$ defined in $(9.2 .15)$ is parameterizable.

Lemma 9.2.3. The disturbance rejection signal $k_{3}^{*}(t)$ defined in (9.2.15) for the state feedback controller (9.2.1) can be parameterized as

$$
k_{3}^{*}(t)=-\frac{Q_{l}(s) Z_{d}(s)+\Theta_{d}^{* T} A_{d}(s)}{\Lambda(s)}[d](t),
$$


where $\Lambda(s)$ is a chosen stable polynomial of degree $n, Q_{l}(s)$ is a polynomial matrix, such that $\frac{Q_{l}(s) Z_{d}(s)}{\Lambda(s)}$ is proper, $\Theta_{d}^{*} \in R^{n q \times M}$ is a constant matrix, $A_{d}(s)=$ $\left[I_{q}, \ldots, s^{n-1} I_{q}\right]^{T}$ with $I_{q}$ being the $q \times q$ identity matrix.

Proof: In order to parameterized $k_{3}^{*}(t)$, we first show that the rational matrix $Z_{l}^{-1}(s)-$ $K_{1}^{* T}(s I-A+L C) B Z_{l}^{-1}(s)$ of $(9.2 .15)$ can be parameterized as $\frac{Q_{l}(s)}{\Lambda(s)}$, for some polynomial matrix $Q_{l}(s)$.

By parameterizing $K_{1}^{* T}(s I-A+L C)^{-1} B$ and $K_{1}^{* T}(s I-A+L C)^{-1} L$ as

$$
\begin{aligned}
& K_{1}^{* T}(s I-A+L C)^{-1} B=\Theta_{1}^{* T} \frac{A_{0}(s)}{\Lambda(s)} \\
& K_{1}^{* T}(s I-A+L C)^{-1} L=\Theta_{2}^{* T} \frac{A_{0}(s)}{\Lambda(s)}
\end{aligned}
$$

where $\operatorname{det}(s I-A+L C)=\Lambda(s)$ being a chosen stable polynomial can be ensured by some $L \in R^{n \times M}$, due to observability of $(A, C), A_{0}(s)=\left[I_{M}, s I_{M}, \ldots, s^{n-1} I_{M}\right]^{T}$ with $I_{M}$ being the $M \times M$ identity matrix, and $\Theta_{1}^{*} \in R^{n M \times M}$ and $\Theta_{2}^{*} \in R^{n M \times M}$ are some constant matrices, we have the matching equation (9.2.7) as

$$
\left(\Lambda(s) I_{M}-\Theta_{1}^{* T} A_{0}(s)\right) Z_{l}^{-1}(s) P_{l}(s)=\Lambda(s) K_{2}^{*} \xi_{m}(s)+\Theta_{2}^{* T} A_{0}(s)
$$

Dividing $\Lambda(s) K_{2}^{*} \xi_{m}(s)+\Theta_{2}^{* T} A_{0}(s)$ on the right by $P_{l}(s)$, we have

$$
\Lambda(s) K_{2}^{*} \xi_{m}(s)+\Theta_{2}^{* T} A_{0}(s)=Q_{l}(s) P_{l}(s)+R_{l}(s)
$$

for some polynomial matrices $Q_{l}(s)$ and $R_{l}(s)$, such that $R_{l}(s) P_{l}^{-1}(s)$ is strictly proper [3]. Substituting (9.2.24) in (9.2.23), we have

$$
\Lambda(s) I_{M}-\Theta_{1}^{* T} A_{0}(s)=Q_{l}(s) Z_{l}(s)+R_{l}(s) P_{l}^{-1}(s) Z_{l}(s) .
$$

Since the left-hand side of the equation (9.2.25) is a polynomial matrix, the righthand side of $(9.2 .25)$ must be a polynomial matrix as well. Moreover, $R_{l}(s) P_{l}^{-1}(s)$ is 
strictly proper, and $P_{l}(s)$ and $Z_{l}(s)$ are coprime as defined in the assumption (A5), we can conclude that $R_{l}(s)$ must be zero to ensure that the right-hand side of the equation (9.2.25) is a polynomial matrix. Therefore, from (9.2.25) with $R_{l}(s)=0$, we have

$$
\left(\Lambda(s) I_{M}-\Theta_{1}^{* T} A_{0}(s)\right) Z_{l}^{-1}(s)=Q_{l}(s) .
$$

From (9.2.21) and (9.2.26), it follows that the rational matrix $Z_{l}^{-1}(s)-K_{1}^{* T}(s I-A+$ $L C) B Z_{l}^{-1}(s)$ of (9.2.15) can be parameterized as

$$
\begin{aligned}
& Z_{l}^{-1}(s)-K_{1}^{* T}(s I-A+L C)^{-1} B Z_{l}^{-1}(s) \\
= & \frac{\left(\Lambda(s) I_{M}-\Theta_{l}^{* T} A_{0}(s)\right) Z_{l}^{-1}(s)}{\Lambda(s)}=\frac{Q_{l}(s)}{\Lambda(s)},
\end{aligned}
$$

where $Q_{l}(s)$ is a polynomial matrix. Then, together with

$$
K_{1}^{* T}(s I-A+L C)^{-1} B_{d}=\Theta_{d}^{* T} \frac{A_{d}(s)}{\Lambda(s)},
$$

for some parameter matrix $\Theta_{d}^{*} \in R^{n q \times M}$ and $A_{d}(s)=\left[I_{q}, s I_{q}, \ldots, s^{n-1} I_{q}\right]^{T}$ with $I_{q}$ being the $q \times q$ identity matrix, we have that $k_{3}^{*}(t)$ in (9.2.15) can be parameterized as

$$
k_{3}^{*}(t)=-\frac{Q_{l}(s) Z_{d}(s)+\Theta_{d}^{* T} A_{d}(s)}{\Lambda(s)}[d](t) .
$$

Since $Z_{l}^{-1}(s) Z_{d}(s)$ is proper from the assumption (A5) and

$$
\frac{Q_{l}(s) Z_{d}(s)}{\Lambda(s)}=\frac{\left(\Lambda(s) I_{M}-\Theta_{1}^{* T} A_{0}(s)\right)}{\Lambda(s)} Z_{l}^{-1}(s) Z_{d}(s)
$$

as from (9.2.27), we have that $\frac{Q_{l}(s) Z_{d}(s)}{\Lambda(s)}$ is proper.

New parametrization of the state feedback controller. Since $\frac{Q_{l}(s) Z_{d}(s)}{\Lambda(s)}$ is proper and $\frac{\Theta_{d}^{* T} A_{d}(s)}{\Lambda(s)}$ is strictly proper as from Lemma 9.2.3, we can further parameterize $k_{3}^{*}(t)$ as

$$
k_{3}^{*}(t)=-\frac{Q_{l}(s) Z_{d}(s)+\Theta_{d}^{* T} A_{d}(s)}{\Lambda(s)}[d](t)=\Theta_{3}^{* T} \frac{A_{d}(s)}{\Lambda(s)}[d](t)+\Theta_{30}^{* T} d(t),
$$


where $\Theta_{3}^{*}=\left[\Theta_{31}^{* T}, \Theta_{32}^{* T}, \ldots, \Theta_{3 n}^{* T}\right]^{T} \in R^{n q \times M}$ with $\Theta_{3 i}^{*} \in R^{q \times M}, i=1,2, \ldots, n$, and $\Theta_{30}^{*} \in R^{q \times M}$ are some constant matrices that depend on $\Theta_{d}^{*}$ and coefficients of the polynomial matrices $Q_{l}(s)$ and $Z_{d}(s)$.

We express the disturbance signal $d(t)=\left[d_{1}(t), d_{2}(t), \ldots, d_{q}(t)\right]^{T}$ as

$$
d_{j}(t)=\bar{d}_{j 0}+\sum_{k=1}^{p_{j}} \bar{d}_{j k} \omega_{j k}(t)=\phi_{j}^{* T} \psi_{j}(t), \quad j=1,2, \ldots, q,
$$

where $\phi_{j}^{*}=\left[\bar{d}_{j 0}, \bar{d}_{j 1}, \ldots, \bar{d}_{j p_{j}}\right]^{T} \in R^{p_{j}+1}$ and $\psi_{j}(t)=\left[1, \omega_{j 1}(t), \ldots, \omega_{j p_{j}}(t)\right]^{T}$. Then we have

$$
d(t)=\Phi^{* T} \Psi(t),
$$

where $\Phi^{*}=\operatorname{diag}\left\{\phi_{1}^{*}, \phi_{2}^{*}, \ldots, \phi_{q}^{*}\right\}$ and $\Psi(t)=\left[\psi_{1}^{T}(t), \psi_{2}^{T}(t), \ldots, \psi_{q}^{T}(t)\right]^{T}$. Substituting (9.2.33) in (9.2.31), we can parameterize $k_{3}^{*}(t)$ as

$$
k_{3}^{*}(t)=K_{3}^{* T} \omega_{3}(t)
$$

where

$$
K_{3}^{*}=\left[\Theta_{31}^{* T} \Phi^{* T}, \ldots, \Theta_{3 n}^{* T} \Phi^{* T}, \Theta_{30}^{* T} \Phi^{* T}\right]^{T}, \omega_{3}(t)=\left[\left(\frac{A_{\psi}(s)}{\Lambda(s)}[\Psi](t)\right)^{T}, \Psi^{T}(t)\right]^{T},
$$

for $A_{\psi}(s)=\left[I_{n_{\psi}}, s I_{n_{\psi}}, \ldots, s^{n-1} I_{n_{\psi}}\right]^{T}$, where $I_{n_{\psi}}$ is the $n_{\psi} \times n_{\psi}$ identity matrix with $n_{\psi}=\sum_{j=1}^{q} p_{j}+q$.

With the new parametrization of $k_{3}^{*}(t)$ in (9.2.34), we obtain the nominal state feedback controller (9.2.1) as

$$
u(t)=K_{1}^{* T} x(t)+K_{2}^{*} r(t)+k_{3}^{*}(t)=K_{1}^{* T} x(t)+K_{2}^{*} r(t)+K_{3}^{* T} \omega_{3}(t),
$$

where $K_{1}^{*}, K_{2}^{*}$, and $K_{3}^{*}$ are unknown due to system and disturbance uncertainties, and the signals $x(t), r(t)$, and $\omega_{3}(t)$ are accessible. 
Remark 9.2.2. If $\frac{Q_{l}(s) Z_{d}(s)}{\Lambda(s)}$ is not proper, we can parameterize the disturbance rejection signal $k_{3}^{*}(t)$ as

$$
\begin{aligned}
k_{3}^{*}(t) & =-\frac{Q_{l}(s) Z_{d}(s)+\Theta_{d}^{* T} A_{d}(s)}{\Lambda(s)}[d](t) \\
& =\Theta_{3}^{* T} \frac{A_{d}(s)}{\Lambda(s)}[d](t)+\Theta_{30}^{* T} d(t)+\Theta_{q}^{* T} A_{q}(s)[d](t),
\end{aligned}
$$

where the proper part of $-\frac{Q_{l}(s) Z_{d}(s)+\Theta_{d}^{* T} A_{d}(s)}{\Lambda(s)}$ is $\Theta_{3}^{* T} \frac{A_{d}(s)}{\Lambda(s)}+\Theta_{30}^{* T}$ and its non-proper part is $\Theta_{q}^{* T} A_{q}(s)$ with $\Theta_{q}^{*}=\left[\Theta_{q 1}^{* T}, \Theta_{q 2}^{* T}, \ldots, \Theta_{q n_{q}}^{* T}\right]^{T} \in R^{n_{q} q \times M}$ and $A_{q}(s)=\left[s I_{q}, \ldots, s^{n_{q}} I_{q}\right]^{T}$ for some order $n_{q}$. With the parametrization of $d(t)$ in (9.2.33), we have

$$
k_{3}^{*}(t)=K_{30}^{* T} \omega_{30}(t)+K_{31}^{* T} \omega_{31}(t),
$$

where

$$
\begin{aligned}
K_{30}^{*} & =\left[\Theta_{31}^{* T} \Phi^{* T}, \Theta_{32}^{* T} \Phi^{* T}, \ldots, \Theta_{3 n}^{* T} \Phi^{* T}, \Theta_{30}^{* T} \Phi^{* T}\right]^{T}, \\
\omega_{30}(t) & =\left[\left(\frac{A_{\psi}(s)}{\Lambda(s)}[\Psi](t)\right)^{T}, \Psi^{T}(t)\right]^{T}, \\
K_{31}^{*} & =\left[\Theta_{q 1}^{* T} \Phi^{* T}, \Theta_{q 2}^{* T} \Phi^{* T}, \ldots, \Theta_{q n_{q}}^{* T} \Phi^{* T}\right]^{T}, \\
\omega_{31}(t) & =\left[s\left[\Psi^{T}\right](t), s^{2}\left[\Psi^{T}\right](t), \ldots, s^{n_{q}}\left[\Psi^{T}\right](t)\right]^{T} .
\end{aligned}
$$

For this linear parametrization of $k_{3}^{*}(t)$, the signal $\omega_{30}(t)$ is accessible, while the accessibility of the signal $\omega_{31}(t)$ needs assumptions that the order $n_{q}$ is known and the $i$ th order derivative $\left(i=1,2, \ldots, n_{q}\right)$ of $\Psi(t)$ can be obtained. If the $i$ th order derivative of $\Psi(t)$ cannot be obtained, we may use

$$
\frac{s^{i}}{(\tau s+1)^{i}}[\Psi](t)
$$

with a small $\tau>0$ to approximate the $i$ th order derivative of $\Psi(t)$. Therefore, when $\frac{Q_{l}(s) Z_{d}(s)}{\Lambda(s)}$ is not proper, the disturbance rejection signal $k_{3}^{*}(t)$ may still be parameterized as the linear combinations of some unknown parameters and known signals. Hence, the nominal controller (9.2.1) can also be parameterized as (9.2.36). 
Based on this new parametrization of the nominal controller (9.2.36), we can proceed the adaptive control design for the system (9.1.1) with unknown parameters and uncertain disturbances.

\subsection{Adaptive State Feedback Control Design}

From the newly parameterized nominal controller (9.2.36), we choose the adaptive controller as

$$
u(t)=K_{1}^{T}(t) x(t)+K_{2}(t) r(t)+K_{3}^{T}(t) \omega_{3}(t),
$$

where $K_{1}(t), K_{2}(t)$, and $K_{3}(t)$ are estimates of $K_{1}^{*}, K_{2}^{*}$, and $K_{3}^{*}$.

Tracking error equation. Substituting the control law (9.3.1) in the system (9.1.1) and from (9.2.34), we have

$$
\begin{aligned}
\dot{x}(t)= & \left(A+B K_{1}^{* T}\right) x(t)+B\left(K_{2}^{*} r(t)+k_{3}^{*}(t)\right)+B_{d} d(t) \\
& +B\left(\widetilde{K}_{1}^{T}(t) x(t)+\widetilde{K}_{2}(t) r(t)+\widetilde{K}_{3}^{T}(t) \omega_{3}(t)\right), \\
y(t)= & C x(t),
\end{aligned}
$$

where $\widetilde{K}_{1}(t)=K_{1}(t)-K_{1}^{*}, \widetilde{K}_{2}(t)=K_{2}(t)-K_{2}^{*}$, and $\widetilde{K}_{3}(t)=K_{3}(t)-K_{3}^{*}$. It follows that

$$
\begin{aligned}
y(t)= & C\left(s I-A-B K_{1}^{* T}\right)^{-1} B K_{2}^{*}[r](t)+C e^{\left(A+B K_{1}^{* T}\right) t} x(0) \\
& +C\left(s I-A-B K_{1}^{* T}\right)^{-1} B\left[k_{3}^{*}\right](t)+C\left(s I-A-B K_{1}^{* T}\right)^{-1} B_{d}[d](t) \\
& +C\left(s I-A-B K_{1}^{* T}\right)^{-1} B\left[\widetilde{\Theta}^{T} \omega\right](t),
\end{aligned}
$$

where $\widetilde{\Theta}(t)=\Theta(t)-\Theta^{*}, \Theta(t)=\left[K_{1}^{T}, K_{2}, K_{3}^{T}\right]^{T}, \Theta^{*}=\left[K_{1}^{* T}, K_{2}^{*}, K_{3}^{* T}\right]^{T}$, and $\omega(t)=$ $\left[x^{T}, r^{T}, \omega_{3}^{T}\right]^{T}$. In view of (9.3.3), from the matching conditions (9.2.2) and (9.2.19), we have the tracking error $e(t)=y(t)-y_{m}(t)$ as

$$
e(t)=W_{m}(s) K_{p}\left[\widetilde{\Theta}^{T} \omega\right](t)+C e^{\left(A+B K_{1}^{* T}\right) t} x(0) .
$$


From the tracking error (9.3.4), ignoring $C e^{\left(A+B K_{1}^{* T}\right) t} x(0)$, we obtain

$$
\xi_{m}(s)[e](t)=K_{p} \widetilde{\Theta}^{T}(t) \omega(t) .
$$

To deal with the uncertainty of the high frequency gain matrix $K_{p}$, we use its LDS decomposition:

$$
K_{p}=L_{s} D_{s} S
$$

where $S \in R^{M \times M}$ with $S=S^{T}>0, L_{s}$ is an $M \times M$ unit lower triangular matrix, and

$$
D_{s}=\operatorname{diag}\left\{\operatorname{sign}\left[\Delta_{1}\right] \gamma_{1}, \ldots, \operatorname{sign}\left[\frac{\Delta_{M}}{\Delta_{M-1}}\right] \gamma_{M}\right\}
$$

with $\Delta_{i}, i=1,2, \ldots, M$, being the $i$ th leading principal minor of $K_{p}$, such that $\gamma_{i}>0$ are arbitrary [84]. Substituting (9.3.6) in (9.3.5), we obtain

$$
L_{s}^{-1} \xi_{m}(s)[e](t)=D_{s} S \widetilde{\Theta}^{T}(t) \omega(t) .
$$

To parameterize the unknown matrix $L_{s}$, we introduce

$$
\Theta_{0}^{*}=L_{s}^{-1}-I=\left\{\theta_{i j}^{*}\right\},
$$

where $\theta_{i j}^{*}=0$ for $i=1,2, \ldots, M$ and $j \geq i$. From (9.3.8) and (9.3.9), we have

$$
\xi_{m}(s)[e](t)+\Theta_{0}^{*} \xi_{m}(s)[e](t)=D_{s} S \widetilde{\Theta}^{T}(t) \omega(t) .
$$

We introduce a filter $h(s)=1 / f(s)$, where $f(s)$ is a stable and monic polynomial whose degree equals to the degree of $\xi_{m}(s)$. Operating both sides of (9.3.10) by $h(s) I_{M}$ leads to

$$
\bar{e}(t)+\left[0, \theta_{2}^{* T} \eta_{2}(t), \theta_{3}^{* T} \eta_{3}(t), \ldots, \theta_{M}^{* T} \eta_{M}(t)\right]^{T}=D_{s} S h(s)\left[\widetilde{\Theta}^{T} \omega\right](t),
$$

where

$$
\begin{aligned}
\bar{e}(t) & =\xi_{m}(s) h(s)[e](t)=\left[\bar{e}_{1}(t), \ldots, \bar{e}_{M}(t)\right]^{T}, \\
\eta_{i}(t) & =\left[\bar{e}_{1}(t), \ldots, \bar{e}_{i-1}(t)\right]^{T}, i=2, \ldots, M, \\
\theta_{i}^{*} & =\left[\theta_{i 1}^{*}, \ldots, \theta_{i i-1}^{*}\right]^{T}, i=2, \ldots, M .
\end{aligned}
$$


Estimation error. Based on this parameterized error, we introduce the estimation error signal

$$
\epsilon(t)=\left[0, \theta_{2}^{T}(t) \eta_{2}(t), \theta_{3}^{T}(t) \eta_{3}(t), \ldots, \theta_{M}^{T}(t) \eta_{M}(t)\right]^{T}+\Psi(t) \xi(t)+\bar{e}(t)
$$

where $\Psi(t)$ is the estimate of $\Psi^{*}=D_{s} S$, and

$$
\xi(t)=\Theta^{T}(t) \zeta(t)-h(s)\left[\Theta^{T} \omega\right](t), \quad \zeta(t)=h(s)[\omega](t)
$$

From (9.3.11)-(9.3.16), we have

$$
\epsilon(t)=\left[0, \tilde{\theta}_{2}^{T}(t) \eta_{2}(t), \tilde{\theta}_{3}^{T}(t) \eta_{3}(t), \ldots, \tilde{\theta}_{M}^{T}(t) \eta_{M}(t)\right]^{T}+D_{s} S \tilde{\Theta}(t)^{T} \zeta(t)+\tilde{\Psi}(t) \xi(t)
$$

where $\tilde{\theta}_{i}(t)=\theta_{i}(t)-\theta_{i}^{*}, i=2,3, \ldots, M$, and $\widetilde{\Psi}(t)=\Psi(t)-\Psi^{*}$ are the related parameter errors.

Adaptive laws. With the estimation error model (9.3.17), we choose

$$
\begin{aligned}
\dot{\theta}_{i}(t) & =-\frac{\Gamma_{\theta i} \epsilon_{i}(t) \eta_{i}(t)}{m^{2}(t)}, i=2,3, \ldots, M, \\
\dot{\Theta}^{T}(t) & =-\frac{D_{s} \epsilon(t) \zeta^{T}(t)}{m^{2}(t)}, \\
\dot{\Psi}(t) & =-\frac{\Gamma \epsilon(t) \xi^{T}(t)}{m^{2}(t)}
\end{aligned}
$$

where the signal $\epsilon(t)=\left[\epsilon_{1}(t), \epsilon_{2}(t), \ldots, \epsilon_{M}(t)\right]^{T}$ is computed from (9.3.15), $\Gamma_{\theta i}=\Gamma_{\theta i}^{T}>$ $0, i=2,3, \ldots, M$, and $\Gamma=\Gamma^{T}>0$ are adaptation gain matrices, and

$$
m(t)=\left(1+\zeta^{T}(t) \zeta(t)+\xi^{T}(t) \xi(t)+\sum_{i=2}^{M} \eta_{i}^{T}(t) \eta_{i}(t)\right)^{1 / 2} .
$$

For the adaptive laws (9.3.18)-(9.3.20), we can have the following properties.

Lemma 9.3.1. The adaptive laws (9.3.18)-(9.3.20) ensure that

(i) $\theta_{i}(t) \in L^{\infty}, i=2,3, \ldots, M, \Theta(t) \in L^{\infty}, \Psi(t) \in L^{\infty}$, and $\frac{\epsilon(t)}{m(t)} \in L^{2} \cap L^{\infty}$;

(ii) $\dot{\theta}_{i}(t) \in L^{2} \cap L^{\infty}, i=2,3, \ldots, M, \dot{\Theta}(t) \in L^{2} \cap L^{\infty}$, and $\dot{\Psi}(t) \in L^{2} \cap L^{\infty}$. 
Proof: Consider the positive definite function

$$
V=\frac{1}{2}\left(\sum_{i=2}^{M} \tilde{\theta}_{i}^{T} \Gamma_{\theta i}^{-1} \tilde{\theta}_{i}+\operatorname{tr}\left[\widetilde{\Psi}^{T} \Gamma^{-1} \widetilde{\Psi}\right]+\operatorname{tr}\left[\widetilde{\Theta} S \widetilde{\Theta}^{T}\right]\right)
$$

From (9.3.18)-(9.3.20), we obtain that the time-derivative of $V$ satisfies

$$
\dot{V}=-\sum_{i=2}^{M} \frac{\tilde{\theta}_{i}^{T} \epsilon_{i}(t) \eta_{i}(t)}{m^{2}(t)}-\frac{\xi^{T}(t) \widetilde{\Psi}^{T} \epsilon(t)}{m^{2}(t)}-\frac{\zeta^{T}(t) \widetilde{\Theta} S D_{s} \epsilon(t)}{m^{2}(t)}=-\frac{\epsilon^{T}(t) \epsilon(t)}{m^{2}(t)} \leq 0 .
$$

From (9.3.22), we have $\theta_{i}(t) \in L^{\infty}, i=2,3, \ldots, M, \Theta(t) \in L^{\infty}, \Psi(t) \in L^{\infty}, \frac{\epsilon(t)}{m(t)} \in$ $L^{2} \cap L^{\infty}, \dot{\theta}_{i}(t) \in L^{2} \cap L^{\infty}, i=2,3, \ldots, M, \dot{\Theta}(t) \in L^{2} \cap L^{\infty}$, and $\dot{\Psi}(t) \in L^{2} \cap L^{\infty} . \nabla$

Based on Lemma 9.3.1, we can prove the following stability properties.

Theorem 9.3.1. The multivariable MRAC scheme with the state feedback controller (9.3.1) updated by the adaptive laws (9.3.18)-(9.3.20), when applied to the system (9.1.1) with unknown parameters and uncertain disturbances, guarantees the closedloop signal boundedness and asymptotic output tracking: $\lim _{t \rightarrow \infty}\left(y(t)-y_{m}(t)\right)=0$, for any initial conditions.

The proof of Theorem 9.3.1 can be carried out by using a similar way as described in [84] for multivariable MRAC using output feedback. We first express the state feedback control signal $u(t)$ in terms of the output signal $y(t)$ by using the state observer representation of the system as given in equations (9.2.8) and (9.2.9). Then, a filtered version of the output $y(t)$ in a feedback framework which has a small gain due to the $L^{2}$ properties of $\dot{\Theta}(t), \dot{\theta}_{i}(t)$ and $\frac{\epsilon(t)}{m(t)}$ can be derived. Hence, the analysis procedure in [84] can be used to conclude the closed-loop signal boundedness and asymptotic output tracking: $\lim _{t \rightarrow \infty}\left(y(t)-y_{m}(t)\right)=0$ for the state feedback case. 


\subsection{Rejection of Output Disturbance and Constant Dynamics Offset}

In this subsection, we will present an adaptive disturbance rejection scheme for MIMO LTI systems with a constant dynamics offset and output disturbances in addition to input disturbances.

Consider the MIMO LTI system (9.1.1) with input disturbances, output disturbances, and a constant dynamics offset:

$$
\begin{aligned}
& \dot{x}(t)=A x(t)+B u(t)+f_{0}+B_{d} d_{u}(t), \\
& y(t)=C x(t)+C_{d} d_{y}(t),
\end{aligned}
$$

where $f_{0}$ is the unknown constant dynamics offset, $d_{u}(t) \in R^{q_{u}}$ and $d_{y}(t) \in R^{q_{y}}$ represent the input and output disturbance signals, whose elements can be characterized as (9.1.2), and $A \in R^{n \times n}, B \in R^{n \times M}, C \in R^{M \times n}, B_{d} \in R^{n \times q_{u}}$, and $C_{d} \in R^{M \times q_{y}}$ are some unknown constant matrices.

In order to design the adaptive control law to cancel the effect of the unknown disturbances and offset on the output signal $y(t)$ and make the output signal $y(t)$ track the reference signal $y_{m}(t)$ generated from (9.1.3), we will first derive the nominal control law by assuming the system parameters are known.

\subsubsection{Nominal State Feedback Control Design}

We choose the nominal state feedback controller as

$$
u(t)=K_{1}^{* T} x(t)+K_{2}^{*} r(t)+k_{30}^{*}+k_{31}^{*}(t)+k_{32}^{*}(t)
$$

where the constant $k_{30}^{*}$ is used to cancel the effect of the offset $f_{0}, k_{31}^{*}(t)$ is to cancel the effect of $d_{u}(t), k_{32}^{*}(t)$ is to cancel the effect of $d_{y}(t)$, and the constant parameters 
$K_{1}^{*}$ and $K_{2}^{*}$ satisfy the matching equation (9.2.2). Substituting (9.4.2) in the system (9.4.1), we obtain the closed-loop system as

$$
\begin{aligned}
y(t)= & C\left(s I-A-B K_{1}^{* T}\right)^{-1} B K_{2}^{*}[r](t)+C e^{\left(A+B K_{1}^{* T}\right) t} x(0) \\
& +C\left(s I-A-B K_{1}^{* T}\right)^{-1} f_{0}\left[u_{s}\right](t)+C\left(s I-A-B K_{1}^{* T}\right)^{-1} B k_{30}^{*}\left[u_{s}\right](t) \\
& +C\left(s I-A-B K_{1}^{* T}\right)^{-1} B_{d}\left[d_{u}\right](t)+C\left(s I-A-B K_{1}^{* T}\right)^{-1} B\left[k_{31}^{*}\right](t) \\
& +C_{d} d_{y}(t)+C\left(s I-A-B K_{1}^{* T}\right)^{-1} B\left[k_{32}^{*}\right](t),
\end{aligned}
$$

where $u_{s}(t)$ is the unit step response. From Lemma 9.2.2, we choose $k_{31}^{*}(t)$ as

$$
\begin{aligned}
k_{31}^{*}(t)= & -\left(Z_{l}^{-1}(s) Z_{d}(s)-K_{1}^{* T}(s I-A+L C)^{-1} B Z_{l}^{-1}(s) Z_{d}(s)\right. \\
& \left.+K_{1}^{* T}(s I-A+L C)^{-1} B_{d}\right)\left[d_{u}\right](t)
\end{aligned}
$$

which can be parameterized as (9.2.34) if $Z_{l}^{-1}(s) Z_{d}(s)$ is proper:

$$
k_{31}^{*}(t)=K_{31}^{* T} \omega_{31}(t)
$$

with the unknown constant $K_{31}^{*}$ and the signal $\omega_{31}(t)=\left[\left(\frac{A_{\Psi}(s)}{\Lambda(s)}[\Psi](t)\right)^{T}, \Psi^{T}(t)\right]^{T}$; or (9.2.38) if $Z_{l}^{-1}(s) Z_{d}(s)$ is not proper:

$$
k_{31}^{*}(t)=K_{311}^{* T} \omega_{311}(t)+K_{312}^{* T} \omega_{312}(t)=K_{31}^{* T} \omega_{31}(t)
$$

with some unknown constants $K_{311}^{*}$ and $K_{312}^{*}$ and the signals

$$
\omega_{311}(t)=\left[\left(\frac{A_{\Psi}(s)}{\Lambda(s)}[\Psi](t)\right)^{T}, \Psi^{T}(t)\right]^{T}, \omega_{312}(t)=\left[s\left[\Psi^{T}\right](t), s^{2}\left[\Psi^{T}\right](t), \ldots, s^{n_{q}}\left[\Psi^{T}\right](t)\right]^{T}
$$

to cancel the effect of $d_{u}(t)$, i.e.,

$$
C\left(s I-A-B K_{1}^{* T}\right)^{-1} B\left[k_{31}^{*}\right](t)+C\left(s I-A-B K_{1}^{* T}\right)^{-1} B_{d}\left[d_{u}\right](t)=0,
$$


From the matching equations (9.2.2) and (9.4.7), we have the closed-loop system $(9.4 .3)$ as

$$
\begin{aligned}
y(t)= & W_{m}(s)[r](t)+C e^{\left(A+B K_{1}^{* T}\right) t} x(0) \\
& +C\left(s I-A-B K_{1}^{* T}\right)^{-1} f_{0}\left[u_{s}\right](t)+W_{m}(s) K_{p} k_{30}^{*}\left[u_{s}\right](t) \\
& +C_{d} d_{y}(t)+W_{m}(s) K_{p}\left[k_{32}^{*}\right](t) .
\end{aligned}
$$

To cancel the effect of $d_{y}(t)$, we choose $k_{32}^{*}(t)$ as

$$
k_{32}^{*}(t)=-K_{2}^{*} \xi_{m}(s) C_{d}\left[d_{y}\right](t)=K_{32}^{* T} \omega_{32}(t),
$$

where $K_{32}^{*} \in R^{(l+1) q_{y} \times M}$ is an unknown constant matrix and

$$
\omega_{32}(t)=\left[d_{y}^{T}(t), s\left[d_{y}^{T}\right](t), \ldots, s^{l}\left[d_{y}^{T}\right](t)\right]^{T}
$$

with $l$ being the degree of $\xi_{m}(s)$. It is worth noting that, for the adaptive control design, the signal $\omega_{32}(t)$ should be available for measurement. If the $i$ th order derivative $(i=1,2, \ldots, l)$ of $d_{y}(t)$ can be obtained, we can access $\omega_{32}(t)$ for the adaptive control design. If the $i$ th order derivative $(i=1,2, \ldots, l)$ of $d_{y}(t)$ cannot be obtained, we may use $\frac{s^{i}}{(\tau s+1)^{i}}\left[d_{y}\right](t)$ with a small $\tau>0$ to approximate the $i$ th order derivative of $d_{y}(t)$ to obtain an approximation of the signal $\omega_{32}(t)$.

From the reference system (9.1.3), the closed-loop system (9.4.8), and the signal $k_{32}^{*}(t)$ as in (9.4.9), we have the output tracking error $e(t)=y(t)-y_{m}(t)$ as

$$
e(t)=C\left(s I-A-B K_{1}^{* T}\right)^{-1} f_{0}\left[u_{s}\right](t)+W_{m}(s) K_{p} k_{30}^{*}\left[u_{s}\right](t)+C e^{\left(A+B K_{1}^{* T}\right) t} x(0) .
$$

Next, we will show that there exists a constant $k_{30}^{*}$ to make $\lim _{t \rightarrow \infty} e(t)=0$. Ignoring the exponentially decaying term $C e^{\left(A+B K_{1}^{* T}\right) t} x(0)$, we have the error equation (9.4.11) in $s$ domain as

$$
e(s)=C\left(s I-A-B K_{1}^{* T}\right)^{-1} \frac{f_{0}}{s}+\frac{1}{s} W_{m}(s) K_{p} k_{30}^{*} .
$$


Since $\operatorname{det}\left(s I-A-B K_{1}^{* T}\right)$ is a stable polynomial, which implies that $\operatorname{det}\left(A+B K_{1}^{* T}\right) \neq$ 0 , i.e., $A+B K_{1}^{* T}$ has full rank, we can apply the Laplace final value theorem to obtain

$$
\lim _{t \rightarrow \infty} e(t)=\lim _{s \rightarrow 0} s e(s)=-C\left(A+B K_{1}^{* T}\right)^{-1} f_{0}+W_{m}(0) K_{p} k_{30}^{*} .
$$

Then, to cancel the effect of $f_{0}$, we choose

$$
k_{30}^{*}=K_{p}^{-1} \xi_{m}(0) C\left(A+B K^{* T}\right)^{-1} f_{0},
$$

which leads to

$$
\lim _{t \rightarrow \infty} e(t)=0 .
$$

In conclusion, when applying the nominal controller (9.4.2) with $K_{1}^{*}$ and $K_{2}^{*}$ satisfying the matching equation $(9.2 .2), k_{30}^{*}$ in $(9.4 .14), k_{31}^{*}(t)$ parameterized as (9.4.5) or (9.4.6), and $k_{32}^{*}(t)$ parameterized as (9.4.9):

$$
u(t)=K_{1}^{* T} x(t)+K_{2}^{*} r(t)+k_{30}^{*}+K_{31}^{* T} \omega_{31}(t)+K_{32}^{* T} \omega_{32}(t)
$$

to the system (9.4.1), the effect of the input and output disturbances and the constant dynamics offset on the output tracking error can be canceled and $\lim _{t \rightarrow \infty}(y(t)-$ $\left.y_{m}(t)\right)=0$.

\subsubsection{Adaptive State Feedback Control Design}

Based on the parameterized nominal controller (9.4.16), we choose the adaptive controller as

$$
u(t)=K_{1}^{T}(t) x(t)+K_{2}(t) r(t)+k_{30}(t)+K_{31}^{T}(t) \omega_{31}(t)+K_{32}^{T}(t) \omega_{32}(t),
$$

where $K_{1}(t), K_{2}(t), k_{30}(t), K_{31}(t)$, and $K_{32}(t)$ are estimates of $K_{1}^{*}, K_{2}^{*}, k_{30}^{*}, K_{31}^{*}$, and $K_{32}^{*}$ for the nominal controller (9.4.16). Similar with the adaptive control design process in Section 9.3, we first obtain the tracking error equation:

$$
e(t)=W_{m}(s) K_{p}\left[\widetilde{\Theta}^{T} \omega\right](t),
$$


where $\widetilde{\Theta}(t)=\Theta(t)-\Theta^{*}, \Theta=\left[K_{1}^{T}, K_{2}, k_{30}, K_{31}^{T}, K_{32}^{T}\right]^{T}, \Theta^{*}=\left[K_{1}^{* T}, K_{2}^{*}, k_{30}^{*}, K_{31}^{* T}, K_{32}^{* T}\right]^{T}$, and $\omega(t)=\left[x^{T}(t), r^{T}(t), 1, \omega_{31}^{T}(t), \omega_{32}^{T}(t)\right]^{T}$, then introduce the estimation error signal as in (9.3.15):

$$
\epsilon(t)=\left[0, \theta_{2}^{T}(t) \eta_{2}(t), \theta_{3}^{T}(t) \eta_{3}(t), \ldots, \theta_{M}^{T}(t) \eta_{M}(t)\right]^{T}+\Psi(t) \xi(t)+\bar{e}(t)
$$

Hence, we choose the adaptive laws as

$$
\begin{aligned}
\dot{\theta}_{i}(t) & =-\frac{\Gamma_{\theta i} \epsilon_{i}(t) \eta_{i}(t)}{m^{2}(t)}, i=2,3, \ldots, M, \\
\dot{\Theta}^{T}(t) & =-\frac{D_{s} \epsilon(t) \zeta^{T}(t)}{m^{2}(t)} \\
\dot{\Psi}(t) & =-\frac{\Gamma \epsilon(t) \xi^{T}(t)}{m^{2}(t)} .
\end{aligned}
$$

It can be proved that the state feedback controller (9.4.17) updated by the adaptive laws (9.4.20)-(9.4.22), when applied to the system (9.4.1), guarantees the closed-loop signal boundedness and asymptotic output tracking: $\lim _{t \rightarrow \infty}\left(y(t)-y_{m}(t)\right)=0$, for any initial conditions. 


\section{Chapter 10}

\section{Adaptive Output Feedback Disturbance Rejection for MIMO Piecewise Linear Systems}

In this chapter, we will present an adaptive output feedback control design framework for multivariable piecewise linear system disturbance rejection problems.

\subsection{Problem Statement}

Consider a MIMO piecewise linear system model:

$$
\begin{aligned}
\dot{x}(t) & =A(t) x(t)+B(t) u(t)+B_{d}(t) d_{u}(t), \\
y(t) & =C(t) x(t),
\end{aligned}
$$

where $x(t) \in R^{n}, y(t) \in R^{M}$, and $u(t) \in R^{M}$ are the state, output, and control input vector signals, $d_{u}(t) \in R^{q_{u}}$ represents the uncertain actuation disturbance signal, whose elements can be characterized as

$$
d_{j}(t)=\bar{d}_{j 0}+\sum_{k=1}^{p_{j}} \bar{d}_{j k} \omega_{j k}(t),
$$


with unknown constants $\bar{d}_{j 0}, \bar{d}_{j k}$, and known signals $\omega_{j k}(t)$, for $j=1,2, \ldots, q, k=$ $1,2, \ldots, p_{j}$. With the signal indicator functions defined as

$$
\chi_{j}(t)=\left\{\begin{array}{cc}
1 & \text { if } x(t) \in \Omega_{j} \\
0 & \text { otherwise }
\end{array}\right.
$$

where $\Omega_{j}, j=1,2, \ldots, q$, are the $j$ th subspace of the operational region $\Omega$ such that $\Omega_{i}^{0} \cap \Omega_{k}^{0}=\{\emptyset\}$ for all $i \neq k$ and $\cup_{j=1}^{p} \Omega_{j}=\Omega$, the system piecewise constant matrices $A(t), B(t), C(t)$, and $B_{d}(t)$ can be expressed as

$$
A(t)=\sum_{i=1}^{p} \chi_{i}(t) A_{i}, B(t)=\sum_{i=1}^{p} \chi_{i}(t) B_{i}, C(t)=\sum_{i=1}^{p} \chi_{i}(t) C_{i}, B_{d}(t)=\sum_{i=1}^{p} \chi_{i}(t) B_{d i}
$$

with some unknown constant matrices $A_{i}, B_{i}, C_{i}$, and $B_{d i}$.

Control objective. The objective is to develop an output feedback control law $u(t)$ for the piecewise linear system (10.1.1) to make all the signals in the closed-loop system bounded and the output $y(t)$ track a reference signal $y_{m}(t)$ as close as possible, with $y_{m}(t)$ generated from a reference system

$$
y_{m}(t)=W_{m}(s)[r](t), W_{m}(s)=\xi_{m}^{-1}(s),
$$

where $W_{m}(s)$ is stable and $r(t)$ is bounded.

Assumptions. To proceed the adaptive control design, we make the following assumptions: (A1) for each system mode $i \in P=\{1,2, \ldots, p\},\left(C_{i}, A_{i}, B_{i}\right)$ is controllable and observable; (A2) $G_{0 i}(s)=C_{i}\left(s I-A_{i}\right)^{-1} B_{i}$ has full rank, all zeros of $G_{0 i}(s)$ are stable with their real parts less than $-\delta$ for some known $\delta>0$; (A3) the observability index $v_{i}$ (or its upper bound) of $G_{0 i}$ is known; (A4) there exists a common modified left interactor matrix $\xi_{m}(s)$ of all $G_{0 i}(s)$, which is known, and the reference transfer matrix is chosen as $W_{m}(s)=\xi_{m}^{-1}(s)$; (A5) all leading principal minors $\Delta_{i j}$, $j=1,2, \ldots, M$, of the high frequency gain matrix $K_{p i}$ of $G_{0 i}(s)$ are nonzero and their 
signs are known; (A6) the transfer matrix $Z_{l i}^{-1}(s) Z_{d i}(s)$ is proper, for the fractional descriptions of the input-output transfer matrix $G_{0 i}(s)=C_{i}\left(s I-A_{i}\right)^{-1} B_{i}$ and the disturbance-output transfer matrix $G_{d i}(s)=C_{i}\left(s I-A_{i}\right)^{-1} B_{d i}$ :

$$
G_{0 i}(s)=P_{l i}^{-1}(s) Z_{l i}(s), \quad G_{d i}(s)=P_{l i}^{-1}(s) Z_{d i}(s)
$$

where $P_{l i}(s), Z_{l i}(s)$, and $Z_{d i}(s)$ are polynomial matrices, and $Z_{l i}(s)$ and $P_{l i}(s)$ are left coprime with row degrees of $P_{l i}(s)$ satisfying $\operatorname{deg}_{r_{j}}\left[P_{l i}(s)\right] \leq \nu_{i}, j=1,2, \ldots, M$, where $\nu_{i}$ is the observability index of $\left(A_{i}, C_{i}\right)$.

\subsection{Output Tracking Error Identity}

When operating within the $i$ th subspace $\Omega_{i}$, for the system transfer matrix $G_{0 i}(s)=$ $C_{i}\left(s I-A_{i}\right)^{-1} B_{i}$, there exist constant parameters $\Theta_{1 i}^{*}, \Theta_{2 i}^{*}, \Theta_{20 i}^{*}$, and $\Theta_{3 i}^{*}$, such that [84]

$$
I_{M}-\Theta_{1 i}^{* T} F_{i}(s)-\left(\Theta_{2 i}^{* T} F_{i}(s)+\Theta_{20 i}^{*}\right) G_{0 i}(s)=\Theta_{3 i}^{*} \xi_{m}(s) G_{0 i}(s), \quad \Theta_{3 i}^{*}=K_{p i}^{-1},
$$

where $F_{i}(s)=\frac{A_{0 i}(s)}{\Lambda_{i}(s)}, \Lambda_{i}(s)$ is a monic and stable polynomial of degree $\nu_{i}-1$ and $A_{0 i}(s)=\left[I_{M}, \ldots, s^{\nu_{i}-2} I_{M}\right]^{T}$ with $\nu_{i}$ being the observability index.

When the system is within the $i$ th subspace $\Omega_{i}$, we have

$$
y(t)=G_{0 i}(s)[u](t)+G_{d i}(s)\left[d_{u}\right](t)+\eta_{3 i}(t)
$$

where the signal $\eta_{3 i}(t)$ is due to system mode switches. Operating both sides of (10.2.1) on $u(t)$ and from (10.2.2), we have

$$
\chi_{i}(t) K_{p i}\left(u(t)-\Theta_{1 i}^{* T} \omega_{1 i}-\Theta_{2 i}^{* T} \omega_{2 i}-\Theta_{20 i}^{*} y-\Theta_{4 i}^{*}(t)+\bar{\eta}_{3 i}(t)\right)=\chi_{i}(t) \xi_{m}(s)[y](t),
$$

where $\omega_{1 i}(t)=F_{i}(s)[u](t), \omega_{2 i}=F_{i}(s)[y](t), \bar{\eta}_{3 i}(t)=\left(\Theta_{2 i}^{* T} F_{i}(s)+\Theta_{20 i}^{*}+\Theta_{3 i}^{*} \xi_{m}(s)\right)\left[\eta_{3 i}\right](t)$, and $\Theta_{4 i}^{*}(t)=-\left(I_{M}-\Theta_{1 i}^{* T} F_{i}(s)\right) Z_{l i}^{-1}(s) Z_{d i}(s)\left[d_{u}\right](t)$, which can be parameterized as

$$
\Theta_{4 i}^{*}(t)=\Theta_{5 i}^{* T} \omega_{5 i}(t)
$$


under the assumption (A6) and a parametrization of the disturbance signal $d_{u}(t)=$ $\Phi^{* T} \Psi(t)$ with $\Theta_{5 i}^{* T}$ and $\omega_{5 i}(t)$ as

$$
\begin{aligned}
\Theta_{5}^{*} & =\left[\Theta_{p 1}^{*} \Phi^{* T}, \Theta_{p 2}^{*} \Phi^{* T}, \ldots, \Theta_{p\left(\bar{\nu}_{0}-1\right)}^{*} \Phi^{* T}, \Theta_{0}^{*} \Phi^{* T}\right]^{T} \\
\omega_{5}(t) & =\left[\left(\frac{A_{\psi}(s)}{\Lambda(s)}[\Psi](t)\right)^{T}, \Psi^{T}(t)\right]^{T}, A_{\psi}(s)=\left[I_{n_{\psi}}, s I_{n_{\psi}}, \ldots, s^{\bar{\nu}_{0}-2} I_{n_{\psi}}\right]^{T} .
\end{aligned}
$$

Summing up both sides of (10.2.3) from $i=1$ to $p$ and from (10.1.5):

$$
\xi_{m}(s)\left[y_{m}\right](t)=\sum_{i=1}^{p} \chi_{i}(t) K_{p i} K_{p i}^{-1} r(t)=\sum_{i=1}^{p} \chi_{i}(t) K_{p i} \Theta_{3 i}^{*} r(t),
$$

we have the tracking error equation as

$$
\begin{aligned}
e(t)= & y(t)-y_{m}(t) \\
= & W_{m}(s)\left[\sum_{i=1}^{p} \chi_{i} K_{p i}\left(u-\Theta_{1 i}^{* T} \omega_{1 i}-\Theta_{2 i}^{* T} \omega_{2 i}-\Theta_{20 i}^{* T} y-\Theta_{3 i}^{*} r-\Theta_{5 i}^{* T} \omega_{5 i}\right)\right](t) \\
& +\eta_{4}(t),
\end{aligned}
$$

where

$$
\eta_{4}(t)=W_{m}(s)\left[\sum_{i=1}^{p} \chi_{i} K_{p i} \bar{\eta}_{3 i}\right](t) .
$$

Remark 10.2.1. Whenever a system mode switch occurs, the part of internal system state $x(t)$, contributed by input $u(t)$ over the past switching time intervals, are not matched by $y_{m}(t)$ at the system output $y(t)$. Such a mismatch of the output signal $y(t)$ and the reference signal $y_{m}(t)$ due to system mode switches is characterized as the term $\eta_{4}(t)$ given by (10.2.9), which is small in the mean square sense under a slow switching condition [81] when applying the nominal controller $u(t)=\sum_{i=1}^{p} \chi_{i}(t) \Theta_{1 i}^{* T} \omega_{1 i}(t)+$ $\sum_{i=1}^{p} \chi_{i}(t) \Theta_{2 i}^{* T} \omega_{2 i}(t)+\sum_{i=1}^{p} \chi_{i}(t) \Theta_{20 i}^{* T} y(t)+\sum_{i=1}^{p} \chi_{i}(t) \Theta_{3 i}^{*} r(t)+\sum_{i=1}^{p} \chi_{i}(t) \Theta_{5 i}^{* T} \omega_{5 i}(t)$. 


\subsection{Adaptive Output Feedback Control Design}

Based on the tracking error equation (10.2.8), we choose the adaptive output feedback controller as

$$
\begin{aligned}
u(t)= & \sum_{i=1}^{p} \chi_{i}(t) \Theta_{1 i}^{T}(t) \omega_{1 i}(t)+\sum_{i=1}^{p} \chi_{i}(t) \Theta_{2 i}^{T}(t) \omega_{2 i}(t)+\sum_{i=1}^{p} \chi_{i}(t) \Theta_{20 i}^{T}(t) y(t) \\
& +\sum_{i=1}^{p} \chi_{i}(t) \Theta_{3 i}^{T}(t) r(t)+\sum_{i=1}^{p} \chi_{i}(t) \Theta_{5 i}^{T}(t) \omega_{5 i}(t)
\end{aligned}
$$

where $\Theta_{1 i}(t), \Theta_{2 i}(t), \Theta_{20 i}(t), \Theta_{3 i}(t)$, and $\Theta_{5 i}(t)$, are the estimates of the nominal parameters $\Theta_{1 i}^{*}, \Theta_{2 i}^{*}, \Theta_{20 i}^{*}, \Theta_{3 i}^{*}$, and $\Theta_{5 i}^{*}$.

Error models. Applying the adaptive controller (10.3.1) to the system (10.1.1), then from the tracking error identity (10.2.8), we have

$$
e(t)=W_{m}(s)\left[\sum_{i=1}^{p} \chi_{i} K_{p i} \tilde{\Theta}_{(i)}^{T} \omega_{(i)}\right](t)+\eta_{4}(t) .
$$

where

$$
\begin{aligned}
\tilde{\Theta}_{(i)}(t) & =\left[\Theta_{1 i}^{T}-\Theta_{1 i}^{* T}, \Theta_{2 i}^{T}-\Theta_{2 i}^{* T}, \Theta_{20 i}^{T}-\Theta_{20 i}^{* T}, \Theta_{3 i}^{T}-\Theta_{3 i}^{* T}, \Theta_{5 i}^{T}-\Theta_{5 i}^{* T}\right]^{T}, \\
\omega_{(i)}(t) & =\left[\omega_{1 i}^{T}(t), \omega_{2 i}^{T}(t), y^{T}(t), r^{T}(t), \omega_{5 i}^{T}(t)\right]^{T}
\end{aligned}
$$

It is worth noting that, when applying the adaptive controller, the effect of the term $\eta_{4}(t)$ on the tracking error $e(t)$ is different from that when applying the nominal controller, for which the tracking error $e(t)$ is proportional to $\eta_{4}(t)$, while for adaptive control in the presence of parameter uncertainties, the tracking error $e(t)$ is proportional to $\eta_{4}(t)$ only in a mean square sense. However, we can show that a normalized version of $\eta_{4}(t)$ is still small in the mean square sense, when the system mode switching frequency is low, with the controller parameters adaptively updated by robustifying adaptive laws, which is critical to closed-loop system stability analysis. 
Substituting the LDS decomposition of $K_{p i}: K_{p i}=L_{s i} D_{s i} S_{i}$ in the error equation (10.3.2), we have the error model parameterized as

$$
\begin{aligned}
& \sum_{i=1}^{p}\left(\left[0, \theta_{(i) 2}^{* T} \eta_{(i) 2}(t), \theta_{(i) 3}^{* T} \eta_{(i) 3}(t), \ldots, \theta_{(i) M}^{* T} \eta_{(i) M}(t)\right]^{T}+\bar{e}_{(i)}(t)\right) \\
= & \sum_{i=1}^{p} D_{s i} S_{i} h(s)\left[\tilde{\Theta}_{(i)}^{T} \chi_{i} \omega_{(i)}\right](t)+d(t) .
\end{aligned}
$$

where $\theta_{(i) j}^{*} \in R^{j-1}, j=2, \ldots, M$, denotes the $j$ th row elements (non-zero part) of $\Theta_{(i) 0}^{*}=L_{s i}^{-1}-I$, and

$$
\begin{aligned}
\bar{e}_{(i)}(t) & =\xi_{m}(s) h(s)\left[\chi_{i} e\right](t)=\left[\bar{e}_{(i) 1}, \bar{e}_{(i) 2}, \ldots, \bar{e}_{(i) M}\right]^{T} \\
\eta_{(i) j}(t) & =\left[\bar{e}_{(i) 1}, \bar{e}_{(i) 2}, \ldots, \bar{e}_{(i) j-1}\right]^{T} \in R^{j-1} \\
d(t) & =\sum_{i=1}^{p} L_{s i}^{-1} h(s)\left[\chi_{i} \xi_{m}(s)\left[\eta_{4}\right]\right](t)-\eta_{1}(t) \\
\eta_{1}(t) & =\sum_{i=1}^{l} L_{s i}^{-1} h(s)\left[\chi_{i} \xi_{m}(s)[e]\right](t)-\sum_{i=1}^{l} L_{s i}^{-1} h(s)\left[\xi_{m}(s)\left[\chi_{i} e\right]\right](t) .
\end{aligned}
$$

Define the estimation error signal

$$
\begin{aligned}
\epsilon(t)= & \sum_{i=1}^{p}\left(\left[0, \theta_{(i) 2}^{T}(t) \eta_{(i) 2}(t), \theta_{(i) 3}^{T}(t) \eta_{(i) 3}(t), \ldots, \theta_{(i) M}^{T}(t) \eta_{(i) M}(t)\right]^{T}\right. \\
& \left.+\bar{e}_{(i)}(t)\right)+\sum_{i=1}^{p} \Psi_{(i)}(t) \xi_{i}(t)
\end{aligned}
$$

where $\theta_{(i) j}(t), j=2,3, \ldots, M$, is the estimate of $\theta_{(i) j}^{*}$, and $\Psi_{(i)}(t)$ is the estimate of $\Psi_{(i)}^{*}=D_{s i} S_{i}$, and

$$
\begin{aligned}
\xi_{i}(t) & =\Theta_{(i)}^{T}(t) \zeta_{i}(t)-h(s)\left[\Theta_{i}^{T} \chi_{i} \omega_{(i)}\right](t), \\
\zeta_{i}(t) & =h(s)\left[\chi_{i} \omega_{(i)}\right](t)
\end{aligned}
$$

Based on (10.3.5) and (10.3.10), we can obtain the following error model:

$$
\begin{aligned}
\epsilon(t)= & \sum_{i=1}^{p}\left(\left[0, \tilde{\theta}_{(i) 2}^{T}(t) \eta_{(i) 2}(t), \tilde{\theta}_{(i) 3}^{T}(t) \eta_{(i) 3}(t), \ldots, \tilde{\theta}_{(i) M}^{T}(t) \eta_{(i) M}(t)\right]^{T}\right) \\
& +\sum_{i=1}^{p}\left(\tilde{\Psi}_{(i)}(t) \xi_{i}(t)+D_{s i} S_{i} \tilde{\Theta}_{(i)}^{T}(t) \zeta_{i}(t)\right)+d(t),
\end{aligned}
$$


where $\tilde{\theta}_{(i) j}(t)=\theta_{(i) j}(t)-\theta_{(i) j}^{*}, \tilde{\Psi}_{(i)}(t)=\Psi_{(i)}(t)-\Psi_{(i)}^{*}(t)$ are the parameter errors. Similarly, the error term $d(t)$ is the unparameterized part of $\epsilon(t)$, due to system mode switches and initial conditions.

Adaptive laws. Then we choose the following adaptive laws to update $\theta_{(i) j}(t)$, $\Theta_{i}(t)$, and $\Psi_{i}(t), i \in\{1,2, \ldots, p\}, j=2,3, \ldots, M$ :

$$
\begin{aligned}
\dot{\theta}_{(i) j}(t) & =-\frac{\Gamma_{\theta_{(i) j}} \eta_{(i) j}(t) \epsilon_{j}(t)}{m^{2}(t)}+f_{(i) j}(t) \\
\dot{\Theta}_{i}^{T}(t) & =-\frac{D_{s i} \epsilon(t) \zeta_{i}^{T}(t)}{m^{2}(t)}+F_{i}(t) \\
\dot{\Psi}_{i}(t) & =-\frac{\Gamma_{i} \epsilon(t) \xi_{i}^{T}(t)}{m^{2}(t)}+H_{i}(t),
\end{aligned}
$$

where $\epsilon(t)=\left[\epsilon_{1}(t), \epsilon_{2}(t), \ldots, \epsilon_{M}(t)\right]^{T}, f_{j}(t), F_{i}(t)$, and $H_{i}(t)$ are the parameter projection or switching- $\sigma$ modification terms, and the adaptation gain matrices $\Gamma_{\theta_{(i) j}}$ and $\Gamma_{i}$ are positive definite and diagonal, and the normalizing signal is $m^{2}(t)=1+m_{s}(t)$ with $m_{s}(t)$ generated from

$$
\dot{m}_{s}(t)=-2 \delta_{0} m_{s}(t)+\|u(t)\|^{2}+\|y(t)\|^{2}, m_{s}(0)=0, \delta_{0}<\delta
$$

where $\delta_{0}<\delta$ for $\delta$ in the assumption (A2).

Stability analysis. First, the parameter estimates from the adaptive laws (10.3.14)(10.3.16) have the desired properties that they are bounded and within their respective parameter bounds: by considering the positive definite function

$$
V=\frac{1}{2} \sum_{j=2}^{M} \tilde{\theta}_{j}^{T} \Gamma_{\theta_{j}}^{-1} \tilde{\theta}_{j}+\sum_{i=1}^{l}\left(\operatorname{tr}\left[\tilde{\Psi}_{i}^{T} \Gamma_{i}^{-1} \tilde{\Psi}_{i}\right]+\operatorname{tr}\left[\tilde{\Theta}_{i} S_{i} \tilde{\Theta}_{i}^{T}\right]\right),
$$

and its time derivative along (10.3.14)-(10.3.16), together with the fact that $\tilde{\theta}_{j}^{T} \Gamma_{\theta_{j}}^{-1} f_{j} \leq$ $0, \operatorname{tr}\left[\tilde{\Psi}_{i}^{T} \Gamma_{i}^{-1} H_{i}\right] \leq 0$, and $\operatorname{tr}\left[\tilde{\Theta}_{i} S_{i} F_{i}\right] \leq 0$, we can obtain that for $z(t)=\frac{\epsilon(t)}{m(t)}, \dot{\theta}_{j}(t), \dot{\Theta}_{i}(t), \dot{\Psi}_{i}(t)$, and some $c, k>0$

$$
\int_{t}^{t+T}\|z(\tau)\|^{2} d \tau \leq c+k \int_{t}^{t+T} \frac{\|d(\tau)\|^{2}}{m^{2}(\tau)} d \tau, \quad \forall t \geq t_{0}, \quad \forall T \geq 0 .
$$


Then, closed-loop signal boundedness can be proved by deriving a feedback structure in terms of some instrumental signals, for the control system whose loop gain is inversely proportional to the minimum switching interval of the piecewise linear system. Under sufficiently slow system mode switches $\left(T_{0} \geq T_{0}^{*}\right.$ for some $\left.T_{0}^{*}>0\right)$, the boundedness of those instrumental signals can be concluded, which in turn implies the closed-loop signal boundedness.

The mean square tracking performance can be obtained by first dividing the integration time interval $[t, t+T]$ into corresponding switching time intervals. The integral of $\|e(t)\|^{2} / m^{2}(t)$ over each interval is upper bounded by a sum of those of $\|\epsilon(t)\|^{2} / m^{2}(t),\left\|\dot{\theta}_{j}(t)\right\|^{2},\left\|\dot{\Theta}_{i}(t)\right\|^{2}$, and $\left\|\dot{\Psi}_{i}(t)\right\|^{2}$. With their respective mean square properties, it can be shown that

$$
\int_{t}^{t+T}\|e(\tau)\|^{2} d \tau \leq c_{2}+c_{3} \int_{t}^{t+T}\|d(\tau)\|^{2} d \tau
$$

for some $c_{2}, c_{3}>0$, from which the tracking performance

$$
\int_{t}^{t+T}\|e(\tau)\|^{2} d \tau \leq C_{1}+K_{1} n_{T}, \quad \forall t \geq t_{0}, \quad \forall T \geq 0
$$

can be established, where $n_{T}$ is the number of system mode switches over $[t, t+T]$. 


\section{Chapter 11}

\section{Feedback-Based Adaptive Damage Detection for MIMO Systems}

This chapter addresses the design, analysis and evaluation of an adaptive feedbackbased stable damage detection scheme applied to aircraft flight systems with structure damage and parameter uncertainties. An aircraft flight system model is presented to capture decoupling and coupling features of the longitudinal and lateral-directional dynamics before and after damage occurs. Two detectors are used to estimate decoupled healthy system parameters and coupled damaged system parameters. Unlike most fault detection schemes which operate under the assumption that all system signals remain bounded under damage conditions, the adaptive damage detection scheme proposed in this chapter is equipped with a stable adaptive feedback controller for damage detection operation to ensure the desired signal boundedness condition and smooth flight. By comparing estimation error residuals between state signals of the detectors and the aircraft system, system damage is detected. Desired adaptive damage detection performance is demonstrated by extensive GTM simulation studies. 


\subsection{Problem Statement}

We denote the nonlinear aircraft system model (2.1.1)-(2.1.9) as

$$
\dot{x}(t)=f(x(t), u(t)), y(t)=C x(t)=[\theta, \psi]^{T},
$$

with the state vector signal being $x(t)=\left[u_{b}, w_{b}, q_{b}, \theta, v_{b}, r_{b}, p_{b}, \phi, \psi\right]^{T}$, where $\left[u_{b}, w_{b}, q_{b}, \theta\right]^{T}$

represents the longitudinal motion and $\left[v_{b}, r_{b}, p_{b}, \phi, \psi\right]^{T}$ represents the lateral-directional motion, and the control input vector signal being $u(t)=\left[d_{e}, d_{t}, d_{r}, d_{a}\right]^{T}$, where $d_{e}, d_{r}$ and $d_{a}$ are elevator, rudder and aileron deflections whose units are degree, and $d_{t}$ is the engine throttle. To investigate the aircraft dynamic characteristics before and after damage occurs, we linearize the nonlinear aircraft system (11.1.1) at a chosen operating point $\left(x_{0}, u_{0}\right)$. Then, the damage detection scheme will be developed based on the linearized aircraft flight system model.

Detector models. To detect the damage, we will first investigate the aircraft flight system before and after damage occurs to show that the longitudinal and lateral-directional dynamics are decoupled for the healthy system and coupled for the damaged system. Based on such a cross-coupling feature, detector models will be constructed to estimate the unknown decoupled parameters for the healthy system and the unknown coupled parameters for the damaged system, by using the state and control input signals of the linearized aircraft system which are required to be bounded before and after damage. With the bounded and sufficiently rich signals, the damage can be detected by observing the response of residuals between the detector signals and the aircraft system signals.

Multivariable adaptive control. To ensure self-stabilization of the aircraft flight system before and after damage occurs, we will apply a multivariable MRAC design to make all the closed-loop signals bounded and the output signal $y(t)$ track 
a desired reference signal $y_{m}(t)$ generated from a reference system:

$$
y_{m}(t)=W_{m}(s)[r](t), W_{m}(s)=\xi_{m}^{-1}(s),
$$

where $r(t)$ is a bounded reference input signal. It is worth noting that, to make the aircraft system before and after damage track a same reference system $W_{m}(s)=$ $\xi_{m}^{-1}(s)$, the interactor matrix $\xi_{m}(s)$ of the aircraft system should be invariant and known before and after damage. Moreover, to design the adaptive control scheme, the signs of leading principal minors of the high frequency gain matrix $K_{p}$ should be known and invariant before and after damage. In the next section, we will show that $\xi_{m}(s)$ and sign information are known and invariant for the generic linearized aircraft system model before and after damage occurs.

\subsection{Modeling of Aircraft Systems with Damage}

Before designing the damage detection scheme, some characteristics of the general aircraft flight system as well as the GTM before and after damage, which are important for the proposed damage detection design, will be investigated based on linearization of the aircraft system.

We choose a wings-level flight condition as the operating point $\left(x_{0}, u_{0}\right)$ for linearizing the aircraft system (11.1.1), where $x_{0}=\left[u_{b 0}, w_{b 0}, 0, \theta_{0}, 0,0,0,0, \psi_{0}\right]^{T}$ and $u_{0}=\left[d_{e 0}, d_{t 0}, d_{r 0}, d_{a 0}\right]^{T}$. The chosen operating point may not be an equilibrium for the aircraft system, since damage causes uncertain structure changes which makes the equilibrium point unknown. Hence, an unknown dynamics offset $f_{0}=f\left(x_{0}, u_{0}\right)$ will be introduced in the linearized aircraft system. In this chapter, we only manipulate the control surfaces elevator and rudder: $\left[d_{e}(t), d_{r}(t)\right]^{T}$ around the operating point, while setting the other control inputs as operating point values: $\left[d_{t}(t), d_{a}(t)\right]^{T}=\left[d_{t 0}, d_{a 0}\right]^{T}$, for the nonlinear aircraft system (11.1.1). Therefore, the linearized aircraft system is 
given as

$$
\Delta \dot{x}(t)=A \Delta x(t)+B \Delta u(t)+f_{0}, \Delta y(t)=C \Delta x(t)
$$

where $\Delta x=x-x_{0}, \Delta u=\left[d_{e}-d_{e 0}, d_{r}-d_{r 0}\right]^{T}$ and $\Delta y=y-C x_{0}$ are state, control input and output signals of the linearized system, $A=\left.\frac{\partial f}{\partial x}\right|_{\left(x_{0}, u_{0}\right)} \in R^{9 \times 9}, B=\left.\frac{\partial f}{\partial u}\right|_{\left(x_{0}, u_{0}\right)} \in$ $R^{9 \times 2}$, and

$$
C=\left[\begin{array}{lllllllll}
0 & 0 & 0 & 1 & 0 & 0 & 0 & 0 & 0 \\
0 & 0 & 0 & 0 & 0 & 0 & 0 & 0 & 1
\end{array}\right] .
$$

Since there exists uncertain damage, the system parameters $\left(A, B, f_{0}\right)$ are unknown and different before and after damage occurs. Assuming the uncertain damage occurs at $t=T_{d}$ with unknown $T_{d}$, we have

$$
\left(A, B, f_{0}\right)=\left\{\begin{array}{cc}
\left(A_{n}, B_{n}, f_{0 n}\right), & t \leq T_{d} \\
\left(A_{d}, B_{d}, f_{0 d}\right), & t>T_{d}
\end{array},\right.
$$

where $\left(A_{n}, B_{n}, f_{0 n}\right)$ denote the unknown healthy system parameters and $\left(A_{d}, B_{d}, f_{0 d}\right)$ denote the unknown damaged system parameters. Without loss of generality, the symbol " $\Delta$ " in Eq. (11.2.1) will be omitted in the following discussions for the linearization-based design, i.e. the linearized aircraft system is expressed as

$$
\dot{x}(t)=A x(t)+B u(t)+f_{0}, \quad y(t)=C x(t) .
$$

Cross-coupling. From the aircraft dynamic equations (2.1.1)-(2.1.9), generic structures of system parameters $(A, B)$ can be obtained.

When there is no damage, the longitudinal and lateral-directional dynamics are decoupled, where the parameters $A$ and $B$ are given as

$$
A=\left[\begin{array}{cc}
A_{n 1}^{(4 \times 4)} & 0^{(4 \times 5)} \\
0^{(5 \times 4)} & A_{n 4}^{(5 \times 5)}
\end{array}\right], B=\left[\begin{array}{cc}
B_{n 1}^{(4 \times 1)} & 0^{(4 \times 1)} \\
0^{(5 \times 1)} & B_{n 4}^{(5 \times 1)}
\end{array}\right],
$$

with

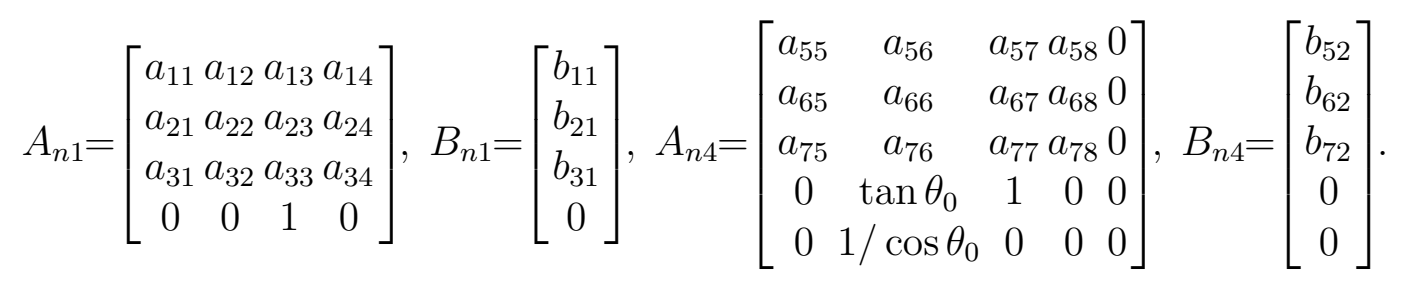


After damage occurs, the longitudinal and lateral-directional dynamics are coupled, where the parameters $A$ and $B$ become to be

$$
A=\left[\begin{array}{ll}
A_{d 1}^{(4 \times 4)} & A_{d 2}^{(4 \times 5)} \\
A_{d 3}^{(5 \times 4)} & A_{d 4}^{(5 \times 5)}
\end{array}\right], B=\left[\begin{array}{ll}
B_{d 1}^{(4 \times 1)} & B_{d 2}^{(4 \times 1)} \\
B_{d 3}^{(5 \times 1)} & B_{d 4}^{(5 \times 1)}
\end{array}\right],
$$

with

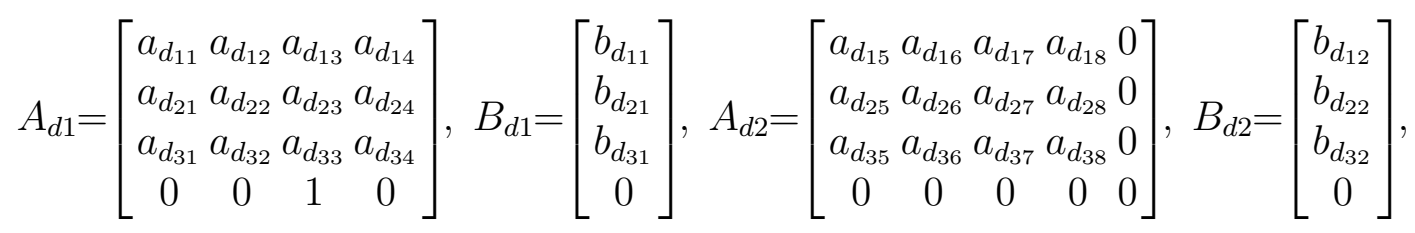

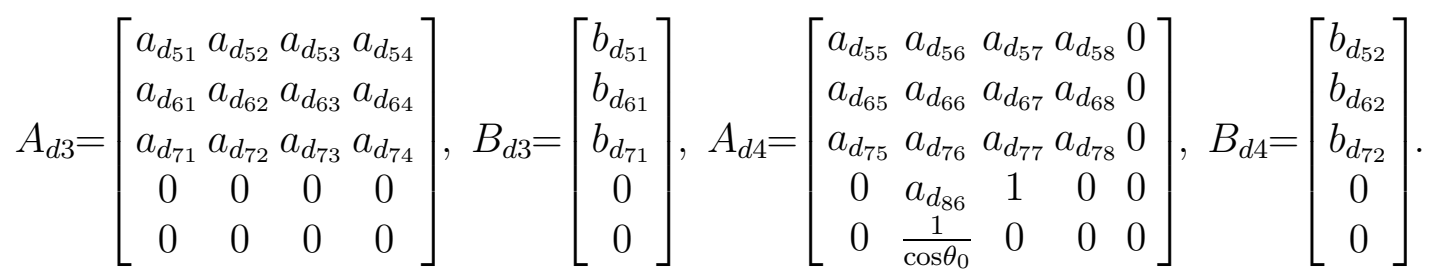

Based on such a cross-coupling characteristic before and after damage, two damage detectors will be established to estimate the block-diagonally decoupled (healthy) and coupled (damaged) system parameters $(A, B)$ to detect the damage.

Invariance properties. Invariance of the infinity zero structure of the aircraft system before and after damage is crucial for the multivariable model reference adaptive control design, since the invariant interactor matrix $\xi_{m}(s)$ ensures that the aircraft system can track a reference system $W_{m}(s)=\xi_{m}^{-1}(s)$ before and after damage occurs and invariant signs of leading principal minors of $K_{p}$ are required for the adaptive control design. To investigate the infinity zero structure for the linearized aircraft system (11.2.4) before and after damage, we first study the relative degrees of entries of the system transfer matrix $G(s)=C(s I-A)^{-1} B$, which can be calculated as

$$
G(s)=\frac{1}{\operatorname{det}(s I-A)}\left(E_{n-1} s^{n-1}+E_{n-2} s^{n-2}+\cdots+E_{0}\right),
$$

where $n$ is the dimension of $A$, $\operatorname{det}(s I-A)=s^{n}+\alpha_{n-1} s^{n-1}+\cdots+\alpha_{1} s+\alpha_{0}, E_{n-1}=$ $C B, E_{n-2}=C A B+\alpha_{n-1} C B, \ldots$, and $E_{0}=C A^{n-1} B+\alpha_{n-1} C A^{n-2} B+\cdots+\alpha_{1} C B$. 
For the healthy aircraft system, where the parameters $A$ and $B$ are given in Eq. (11.2.5) and $C$ is given in Eq. (11.2.2), the coefficients $E_{n-1}$ and $E_{n-2}$ for $G(s)$ are calculated as

$$
E_{n-1}=C B=0, \quad E_{n-2}=C A B=\operatorname{diag}\left\{b_{31}, \frac{b_{61}}{\cos \theta_{0}}\right\}
$$

Based on Lemma 1, the interactor matrix for $G(s)$ can be chosen as

$$
\xi_{m}(s)=\operatorname{diag}\left\{(s+1)^{2},(s+1)^{2}\right\}
$$

it follows that the high frequency gain matrix is given as

$$
K_{p}=\lim _{s \rightarrow \infty} \xi_{m}(s) G(s)=C A B=\operatorname{diag}\left\{b_{31}, \frac{b_{61}}{\cos \theta_{0}}\right\}
$$

Since the parameters $b_{31}$ and $b_{61}$ are the control gains from elevator to pitch acceleration and rudder to yaw acceleration, the signs of these parameters can be obtained: $b_{31}<0$ and $b_{61}<0$. Hence, signs of leading principal minors are given as

$$
\operatorname{sign}\left(\Delta_{1}\right)=\operatorname{sign}\left(b_{31}\right)=-1, \operatorname{sign}\left(\Delta_{2}\right)=\operatorname{sign}\left(\frac{b_{31} b_{61}}{\cos \theta_{0}}\right)=1
$$

After damage occurs, the matrices $A$ and $B$ change to the damaged values given in Eq. (11.2.6). Hence, the coefficients $E_{n-1}$ and $E_{n-2}$ are calculated as

$$
E_{n-1}=0, \quad E_{n-2}=C A B=\left[\begin{array}{cc}
b_{d_{31}} & b_{d_{32}} \\
\frac{1}{\cos \theta_{0}} b_{d_{61}} & \frac{1}{\cos \theta_{0}} b_{d_{62}}
\end{array}\right] .
$$

The interactor matrix of $G(s)$ can be chosen as

$$
\xi_{m}(s)=\operatorname{diag}\left\{(s+1)^{2},(s+1)^{2}\right\},
$$

and the high frequency gain matrix is

$$
K_{p}=\lim _{s \rightarrow \infty} \xi_{m}(s) G(s)=E_{n-2}=\left[\begin{array}{cc}
b_{d_{31}} & b_{d_{32}} \\
\frac{1}{\cos \theta_{0}} b_{d_{61}} & \frac{1}{\cos \theta_{0}} b_{d_{62}}
\end{array}\right] .
$$


If the shift of center of mass is small, the signs of $b_{d_{31}}$ and $b_{d_{62}}$ may still be negative, and the coupling terms may be very small. Therefore, signs of leading principal minors are still

$$
\operatorname{sign}\left(\Delta_{1}\right)=-1, \operatorname{sign}\left(\Delta_{2}\right)=1
$$

From the above generic structure analysis, it follows that the infinity zero structure is invariant before and after damage, which is $\xi_{m}(s)=\operatorname{diag}\left\{(s+1)^{2},(s+1)^{2}\right\}$, and the signs of the leading principal minors of high frequency matrix are invariant when the shift of center of mass is small, which are $\operatorname{sign}\left(\Delta_{1}\right)=-1$ and $\operatorname{sign}\left(\Delta_{2}\right)=1$.

Summary. Since the above analysis is based on the generic aircraft model (11.2.4) linearized at an arbitrarily given wings-level flight operating point, the coupling feature and the invariance properties hold for any general aircraft systems linearized at a wings-level flight operating condition. In the GTM simulation study, we will assess the developed detection scheme around several different operating conditions.

\subsection{Feedback-Based Damage Detection Scheme}

In this section, we will present the detailed adaptive feedback-based damage detection design based on the cross-coupling and invariance properties of the linearized aircraft system model (11.2.4). To build the detector models, the aircraft state signal and control input signal are required to be bounded. Therefore, a self-stabilization feedback control will be developed to ensure the signal boundedness requirement before and after damage occurs.

\subsubsection{Self-Stabilization Feedback Control}

To achieve the closed-loop signal boundedness and output tracking objectives, we apply the multivariable model reference adaptive control scheme to the aircraft system 
(11.2.4) with damage.

State feedback controller structure. To compensate the uncertainties of the system parameters $A$ and $B$, and the unknown constant offset term $f_{0}$ in (11.2.4) before and after damage, we choose the state feedback controller structure as

$$
u(t)=K_{1}^{T}(t) x(t)+K_{2}(t) r(t)+k_{3}(t)
$$

Plant-model matching. The parameters $K_{1}(t), K_{2}(t)$, and $k_{3}(t)$ in (11.3.1) are the adaptively updated estimates of the nominal parameters $K_{1}^{*}, K_{2}^{*}$, and $k_{3}^{*}$ satisfying matching conditions [37]

$$
C\left(s I-A-B K_{1}^{* T}\right)^{-1} B K_{2}^{*}=W_{m}(s), \quad K_{2}^{*-1}=K_{p}, \quad k_{3}^{*}=-D^{-1} d,
$$

with $K_{p}$ being the piecewise constant high frequency gain matrix $K_{p}=\lim _{s \rightarrow \infty} \xi_{m}(s) G(s)$, and $D=-C\left(A+B K_{1}^{*}\right)^{-1} B$ and $d=-C\left(A+B K_{1}^{*}\right)^{-1} f_{0}$. Since the parameters $\left(A, B, f_{0}\right)$ of the system (11.2.4) are piecewise constants due to damage, the nominal parameters $K_{1}^{*}, K_{2}^{*}$, and $k_{3}^{*}$ are piecewise constants before and after damage occurs. From the matching conditions (11.3.2), applying the nominal controller

$$
u(t)=K_{1}^{* T} x(t)+K_{2}^{*} r(t)+k_{3}^{*}
$$

to the system (11.2.4), we have that the closed-loop signals are bounded and the output signal $y(t)$ tracks the reference signal $y_{m}(t)=W_{m}(s)[r](t): \lim _{t \rightarrow \infty}\left(y(t)-y_{m}(t)\right)=$ 0 . However, since the parameters $K_{1}^{*}, K_{2}^{*}$, and $k_{3}^{*}$ in (11.3.2) are unknown due to the uncertainties of the aircraft system, we will apply the adaptively updated control law (11.3.1) to the aircraft system (11.2.4) with damage. To derive the adaptive laws, we first parameterize the tracking error equation.

Tracking error equation. From the reference model (11.1.2), the aircraft system (11.2.4) with the control law (11.3.1) and the matching conditions (11.3.2), we have 
that the output tracking error $e(t)=y(t)-y_{m}(t)$ can be parameterized as

$$
e(t)=W_{m}(s) K_{p}\left[\widetilde{\Theta}^{T} \omega\right](t)
$$

where $\widetilde{\Theta}(t)=\Theta(t)-\Theta^{*}, \Theta(t)=\left[K_{1}^{T}(t), K_{2}(t), k_{3}(t)\right]^{T}, \Theta^{*}=\left[K_{1}^{* T}, K_{2}^{*}, k_{3}^{*}\right]^{T}$, and $\omega(t)=$ $\left[x^{T}(t), r^{T}(t), 1\right]^{T}$. To deal with the uncertainty of $K_{p}$, we use its LDS decomposition $K_{p}=L_{s} D_{s} S$, where $S=S^{T}>0, L_{s}$ is a unit lower triangular matrix, and $D_{s}=$ $\operatorname{diag}\left\{\operatorname{sign}\left[\Delta_{1}\right] \gamma_{1}, \ldots, \operatorname{sign}\left[\frac{\Delta_{M}}{\Delta_{M-1}}\right] \gamma_{M}\right\}$ with arbitrarily chosen $\gamma_{i}>0, i=1, \ldots, M[84]$, where $M$ is the dimension of the input signal $u(t)$. Since the signs of the leading principal minors $\Delta_{i}, i=1,2, \ldots, M$, are invariant before and after damage occurs, we can choose a uniform $D_{s}$ before and after damage as a gain matrix which will be used in the adaptive laws. Substituting the LDS decompensation in (11.3.4) and operating both sides of (11.3.4) by $h(s) I_{M}$, where $h(s)=1 / f_{h}(s)$ with $f_{h}(s)$ being a stable and monic polynomial of degree equals to the degree of $\xi_{m}(s)$, we have

$$
L_{s}^{-1} \xi_{m}(s) h(s)[e](t)=D_{s} S h(s)\left[\tilde{\Theta}^{T} \omega\right](t)
$$

To parameterize the unknown matrix $L_{s}$, we introduce $\Theta_{0}^{*}=L_{s}^{-1}-I=\left\{\theta_{i j}^{*}\right\}$, where $\theta_{i j}^{*}=0$ for $i=1,2, \ldots, M$ and $j \geq i$. Then we have

$$
\bar{e}(t)+\left[0, \theta_{2}^{* T} \eta_{2}(t), \ldots, \theta_{M}^{* T} \eta_{M}(t)\right]^{T}=D_{s} S h(s)\left[\tilde{\Theta}^{T} \omega\right](t),
$$

where $\bar{e}\left(t=\xi_{m}(s) h(s)[e](t)=\left[\bar{e}_{1}(t), \ldots, \bar{e}_{M}(t)\right]^{T}, \eta_{i}(t)=\left[\bar{e}_{1}(t), \ldots, \bar{e}_{i-1}(t)\right]^{T}\right.$, and $\theta_{i}^{*}=\left[\theta_{i 1}^{*}, \ldots, \theta_{i i-1}^{*}\right]^{T}$.

Estimation error. We introduce an estimation error signal

$$
\epsilon(t)=\left[0, \theta_{2}^{T}(t) \eta_{2}(t), \ldots, \theta_{M}^{T}(t) \eta_{M}(t)\right]^{T}+\Psi(t) \xi(t)+\bar{e}(t)
$$

where $\theta_{i}(t), i=2,3, \ldots, M$ are the estimates of $\theta_{i}^{*}$, and $\Psi(t)$ is the estimate of $\Psi^{*}=$ $D_{s} S$, and

$$
\xi(t)=\Theta^{T}(t) \zeta(t)-h(s)\left[\Theta^{T} \omega\right](t), \zeta(t)=h(s)[\omega](t) .
$$


From (11.3.6)-(11.3.8), we can derive that

$$
\epsilon(t)=\left[0, \tilde{\theta}_{2}^{T}(t) \eta_{2}(t), \tilde{\theta}_{3}^{T}(t) \eta_{3}(t), \ldots, \tilde{\theta}_{M}^{T}(t) \eta_{M}(t)\right]^{T}+D_{s} S \tilde{\Theta}^{T}(t) \zeta(t)+\tilde{\Psi}(t) \xi(t),
$$

where $\tilde{\theta}_{i}(t)=\theta_{i}(t)-\theta_{i}^{*}$ and $\tilde{\Psi}(t)=\Psi(t)-\Psi^{*}$.

Adaptive laws. With the estimation error model (11.3.9), we choose

$$
\begin{aligned}
\dot{\theta}_{i}(t) & =-\frac{\Gamma_{\theta i} \epsilon_{i}(t) \eta_{i}(t)}{m^{2}(t)}, i=2,3, \ldots, M \\
\dot{\Theta}^{T}(t) & =-\frac{D_{s} \epsilon(t) \zeta^{T}(t)}{m^{2}(t)} \\
\dot{\Psi}(t) & =-\frac{\Gamma \epsilon(t) \xi^{T}(t)}{m^{2}(t)}
\end{aligned}
$$

where the signal $\epsilon(t)=\left[\epsilon_{1}(t), \epsilon_{2}(t), \ldots, \epsilon_{M}(t)\right]^{T}$ is computed from (11.3.7), $\Gamma_{\theta i}=\Gamma_{\theta i}^{T}>$ $0, i=2,3, \ldots, M$, and $\Gamma=\Gamma^{T}>0$ are adaptation gain matrices, and

$$
m(t)=\left(1+\zeta^{T}(t) \zeta(t)+\xi^{T}(t) \xi(t)+\sum_{i=2}^{M} \eta_{i}^{T}(t) \eta_{i}(t)\right)^{1 / 2}
$$

is a standard normalization signal.

Stability analysis. The multivariable MRAC scheme with the state feedback control law (11.3.1) updated by the adaptive laws (11.3.10)-(11.3.12), when applied to (11.2.4), guarantees the closed-loop signal boundedness and asymptotic output tracking: $\lim _{t \rightarrow \infty}\left(y(t)-y_{m}(t)\right)=0$, for any initial conditions.

\subsubsection{Adaptive Damage Detection}

In this section, we will present two detector models constructed by using the bounded control input signal $u(t)$ and state signal $x(t)$. The key feature of aircraft system before and after damage is that the system parameters $A$ and $B$ are block-diagonally decoupled before damage and coupled after damage. To detect the damaged status, the parameters $A$ and $B$ will be adaptively estimated by the proposed detector models. 
With some sufficiently rich input signals, the damage can be detected by observing the residuals between the state signals of the detectors and the state signals of the aircraft system.

\subsubsection{Detector for healthy aircraft system}

Based on the healthy aircraft model (11.2.4) with decoupled system parameters $(A, B)$ given in (11.2.5), we choose the detector model as

$$
\dot{x}_{m}^{(1)}(t)=A_{m} x_{m}^{(1)}(t)+\left(\widehat{A}^{(1)}(t)-A_{m}\right) x(t)+\widehat{B}^{(1)}(t) u(t)+\hat{f}_{0}^{(1)}(t)
$$

where $x(t)$ and $u(t)$ are the state and input signals of the aircraft system (11.2.4) which are bounded, $A_{m}=\operatorname{diag}\left\{A_{m 1}^{(4 \times 4)}, A_{m 2}^{(5 \times 5)}\right\}$ is a chosen stable matrix, and

$$
\widehat{A}^{(1)}=\left[\begin{array}{cc}
\widehat{A}_{11}^{(1)} & 0 \\
0 & \widehat{A}_{22}^{(1)}
\end{array}\right], \widehat{B}^{(1)}=\left[\begin{array}{cc}
\widehat{B}_{11}^{(1)} & 0 \\
0 & \widehat{B}_{22}^{(1)}
\end{array}\right], \hat{f}_{0}^{(1)}=\left[\begin{array}{c}
\hat{f}_{01}^{(1)} \\
\hat{f}_{02}^{(1)}
\end{array}\right]
$$

are the estimates of healthy aircraft system parameters.

Adaptive laws. The residual between the detector state signal $x_{m}^{(1)}(t)$ and the linearized aircraft state signal $x(t)$ is defined as $e_{m}^{(1)}(t)=x_{m}^{(1)}(t)-x(t)$. For designed adaptive laws, we partition $u(t), x(t)$ and $e_{m}^{(1)}(t)$ into

$$
\begin{aligned}
u(t) & =\left[u_{1}(t), u_{2}(t)\right]^{T}=\left[d_{e}(t), d_{r}(t)\right]^{T}, \\
x(t) & =\left[x_{1}^{T}(t), x_{2}^{T}(t)\right]^{T}, \\
e_{m}^{(1)}(t) & =\left[e_{m 1}^{(1) T}(t), e_{m 2}^{(1) T}(t)\right]^{T},
\end{aligned}
$$

where $x_{1}(t) \in R^{4}, x_{2}(t) \in R^{5}, e_{m 1}^{(1)}(t) \in R^{4}$, and $e_{m 2}^{(1)}(t) \in R^{5}$. Then, we choose adaptive laws for $\widehat{A}_{11}^{(1)}(t), \widehat{A}_{22}^{(1)}(t), \widehat{B}_{11}^{(1)}(t), \widehat{B}_{22}^{(1)}(t), \hat{f}_{01}^{(1)}(t)$, and $\hat{f}_{02}^{(1)}(t)$ as

$$
\begin{aligned}
& \dot{\hat{A}}_{11}^{(1)}(t)=-\Gamma_{1} P_{1} e_{m 1}^{(1)}(t) x_{1}^{T}(t), \dot{\hat{A}}_{22}^{(1)}(t)=-\Gamma_{2} P_{2} e_{m 2}^{(1)}(t) x_{2}^{T}(t), \\
& \dot{\widehat{B}}_{11}^{(1)}(t)=-\Gamma_{3} P_{1} e_{m 1}^{(1)}(t) u_{1}(t), \dot{\widehat{B}}_{22}^{(1)}(t)=-\Gamma_{4} P_{2} e_{m 2}^{(1)}(t) u_{2}(t), \\
& \dot{\hat{f}}_{01}^{(1)}(t)=-\Gamma_{5} P_{1} e_{m 1}^{(1)}(t), \quad \dot{\hat{f}}_{02}^{(1)}(t)=-\Gamma_{6} P_{2} e_{m 2}^{(1)}(t),
\end{aligned}
$$


where $\Gamma_{i}, i=1,2, \ldots, 6$ are symmetric positive definite gain matrices, $P_{1}=P_{1}^{T}>0$ and $P_{2}=P_{2}^{T}>0$, such that $P_{1} A_{m 1}+A_{m 1}^{T} P_{1}=-Q_{1}$ and $P_{2} A_{m 2}+A_{m 2}^{T} P_{2}=-Q_{2}$ with $Q_{1}=Q_{1}^{T}>0$ and $Q_{2}=Q_{2}^{T}>0$.

With the adaptive laws (11.3.17)-(11.3.19) and the bounded state signal $x(t)$ and control input signal $u(t)$ from the closed-loop linearized aircraft system, the residual $e_{m}^{(1)}(t)=x_{m}^{(1)}(t)-x(t)$ has the following properties before and after damage occurs.

Proposition 11.3.1. When there is no damage, the residual $e_{m}^{(1)}(t)=x_{m}^{(1)}(t)-x(t)$ between the detector model (11.3.13) updated by (11.3.17)-(11.3.19) and the linearized aircraft system (11.2.4) with the adaptive controller (11.3.1) updated by (11.3.10)(11.3.12) satisfies that $\lim _{t \rightarrow \infty} e_{m}^{(1)}(t)=0$.

Proof: When there is no damage, the longitudinal and lateral-directional dynamics are decoupled:

$$
\dot{x}(t)=A x(t)+B u(t)+f_{0},
$$

where $A=\operatorname{diag}\left\{A_{n 1}, A_{n 4}\right\}$ and $B=\operatorname{diag}\left\{B_{n 1}, B_{n 4}\right\}$ given in Eq. (11.2.5). Consider a positive definite function:

$$
\begin{aligned}
V= & e_{m 1}^{(1) T} P_{1} e_{m 1}^{(1)}+e_{m 2}^{(1) T} P_{2} e_{m 2}^{(1)}+\operatorname{tr}\left[\widetilde{A}_{11}^{(1) T} \Gamma_{1}^{-1} \widetilde{A}_{11}^{(1)}\right] \\
& +\operatorname{tr}\left[\widetilde{A}_{22}^{(1) T} \Gamma_{2}^{-1} \widetilde{A}_{22}^{(1)}\right]+\widetilde{B}_{11}^{(1) T} \Gamma_{3}^{-1} \widetilde{B}_{11}^{(1)}+\widetilde{B}_{22}^{(1) T} \Gamma_{4}^{-1} \widetilde{B}_{22}^{(1)} \\
& +\tilde{f}_{01}^{(1) T} \Gamma_{5}^{-1} \tilde{f}_{01}^{(1)}+\tilde{f}_{02}^{(1) T} \Gamma_{6}^{-1} \tilde{f}_{02}^{(1)},
\end{aligned}
$$

with $\widetilde{A}_{11}^{(1)}=\widehat{A}_{11}^{(1)}-A_{n 1}, \widetilde{A}_{22}^{(1)}=\widehat{A}_{22}^{(1)}-A_{n 4}, \widetilde{B}_{11}^{(1)}=\widehat{B}_{11}^{(1)}-B_{n 1}, \widetilde{B}_{22}^{(1)}=\widehat{B}_{22}^{(1)}-B_{n 4}$, and $\tilde{f}_{01}^{(1)}=\hat{f}_{01}^{(1)}-f_{01}$ and $\tilde{f}_{01}^{(1)}=\hat{f}_{01}^{(1)}-f_{01}$, where $\left[f_{01}^{T}, f_{02}^{T}\right]^{T}=f_{0}$. From the linearized aircraft system (11.3.20) without damage and the detector model (11.3.13), we have the residual dynamics as

$$
\left[\begin{array}{c}
\dot{e}_{m 1}^{(1)} \\
\dot{e}_{m 2}^{(1)}
\end{array}\right]=\left[\begin{array}{c}
A_{m 1} e_{m 1}^{(1)} \\
A_{m 2} e_{m 2}^{(1)}
\end{array}\right]+\left[\begin{array}{c}
\widetilde{A}_{11}^{(1)} x_{1} \\
\widetilde{A}_{22}^{(1)} x_{2}
\end{array}\right]+\left[\begin{array}{c}
\widetilde{B}_{11}^{(1)} u_{1} \\
\widetilde{B}_{22}^{(1)} u_{2}
\end{array}\right]+\left[\begin{array}{c}
\tilde{f}_{01}^{(1)} \\
\widetilde{f}_{02}^{(1)}
\end{array}\right] .
$$


From the adaptive laws (11.3.17)-(11.3.18), we obtain

$$
\dot{V}=-e_{m 1}^{(1) T}(t) Q_{1} e_{m 1}^{(1)}(t)-e_{m 2}^{(1) T}(t) Q_{2} e_{m 2}^{(1)}(t) \leq 0
$$

Then, we conclude that $e_{m}^{(1)}(t)=\left[e_{m 1}^{(1) T}(t), e_{m 2}^{(1) T}(t)\right]^{T}, \widetilde{A}_{11}^{(1)}(t), \widetilde{A}_{22}^{(1)}(t), \widetilde{B}_{11}^{(1)}(t), \widetilde{B}_{22}^{(1)}(t)$, $\tilde{f}_{01}^{(1)}(t)$, and $\tilde{f}_{02}^{(1)}(t)$ are bounded. Since the state signal $x(t)=\left[x_{1}^{T}(t), x_{2}^{T}(t)\right]^{T}$ and the control input signal $u(t)=\left[u_{1}(t), u_{2}(t)\right]^{T}$ are bounded, we further have $\dot{e}_{m}^{(1)}(t)=$ $\left[\dot{e}_{m 1}^{(1) T}(t), \dot{e}_{m 2}^{(1) T}(t)\right]^{T} \in L^{\infty}$. Eq. (11.3.23) implies that $e_{m}^{(1)}(t)=\left[e_{m 1}^{(1) T}(t), e_{m 2}^{(1) T}(t)\right]^{T} \in L^{2}$. With $e_{m}^{(1)}(t) \in L^{2} \cap L^{\infty}$ and $\dot{e}_{m}^{(1)}(t) \in L^{\infty}$, applying Barbalat lemma, we have that $\lim _{t \rightarrow \infty} e_{m}^{(1)}(t)=0$.

When damage occurs, the parameters $(A, B)$ of linearized aircraft system (11.2.4) are coupled as given in Eq. (11.2.6). Then, we obtain the residual dynamics as

$$
\left[\begin{array}{c}
\dot{e}_{m 1}^{(1)} \\
\dot{e}_{m 2}^{(1)}
\end{array}\right]=\left[\begin{array}{c}
A_{m 1} e_{m 1}^{(1)} \\
A_{m 2} e_{m 2}^{(1)}
\end{array}\right]+\left[\begin{array}{c}
\widetilde{A}_{11}^{(1)} x_{1} \\
\widetilde{A}_{22}^{(1)} x_{2}
\end{array}\right]+\left[\begin{array}{c}
\widetilde{B}_{11}^{(1)} u_{1} \\
\widetilde{B}_{22}^{(1)} u_{2}
\end{array}\right]+\left[\begin{array}{c}
\tilde{f}_{01}^{(1)} \\
\tilde{f}_{02}^{(1)}
\end{array}\right]-\left[\begin{array}{c}
A_{d 2} x_{2} \\
A_{d 3} x_{1}
\end{array}\right]-\left[\begin{array}{c}
B_{d 2} u_{2} \\
B_{d 3} u_{1}
\end{array}\right]
$$

with $\widetilde{A}_{11}^{(1)}=\widehat{A}_{11}^{(1)}-A_{d 1}, \widetilde{A}_{22}^{(1)}=\widehat{A}_{22}^{(1)}-A_{d 4}, \widetilde{B}_{11}^{(1)}=\widehat{B}_{11}^{(1)}-B_{d 1}$, and $\widetilde{B}_{22}^{(1)}=\widehat{B}_{22}^{(1)}-B_{d 4}$. Hence, based on the adaptive laws (11.3.17)-(11.3.18), we have

$$
\begin{aligned}
\dot{V} & =-e_{m 1}^{(1) T} Q_{1} e_{m 1}^{(1)}-e_{m 2}^{(1) T} Q_{2} e_{m 2}^{(1)}-2 e_{m 1}^{(1) T} P_{1} A_{d 2} x_{2} \\
& -2 e_{m 1}^{(1) T} P_{1} B_{d 2} u_{2}-2 e_{m 2}^{(1) T} P_{2} A_{d 3} x_{1}-2 e_{m 2}^{(1) T} P_{2} B_{d 3} u_{1}
\end{aligned}
$$

where the coupling terms may prevent $\dot{V} \leq 0$, that is the residual is not guaranteed to converge to zero in the presence of damage. To further investigate the performance of residual after damage occurs, we have the following proposition.

Proposition 11.3.2. When damage occurs, if each element $u_{i}, i=1,2$ of control input $u$ is sufficiently rich of order $n+1$ and uncorrelated, i.e., each $u_{i}$ contains different frequencies, where $n$ is the dimension of the aircraft system state signal, then the residual $e_{m}^{(1)}(t)=x_{m}^{(1)}(t)-x(t)$, between the detector model (11.3.13) updated 
by (11.3.17)-(11.3.19) and the aircraft system (11.2.4) with the adaptive controller (11.3.1) updated by (11.3.10)-(11.3.12), does not converge to 0.

Proof: When damage occurs, we have the residual dynamics as (11.3.24). Defining

$$
\begin{aligned}
& \tilde{\theta}_{1}^{(1)}=\left[\tilde{a}_{11}^{(1) T}, \tilde{a}_{12}^{(1) T}, \ldots, \tilde{a}_{14}^{(1) T}, \widetilde{B}_{11}^{(1) T}\right]^{T}, \\
& \tilde{\theta}_{2}^{(1)}=\left[\tilde{a}_{21}^{(1) T}, \tilde{a}_{22}^{(1) T}, \ldots, \tilde{a}_{25}^{(1) T}, \widetilde{B}_{22}^{(1) T}\right]^{T},
\end{aligned}
$$

where $\tilde{a}_{1 i}^{(1)}$ is the $i$ th column of $\widetilde{A}_{11}^{(1)}$ and $\tilde{a}_{2 i}^{(1)}$ is the $i$ th column of $\widetilde{A}_{22}^{(1)}$, we can write

$$
\begin{aligned}
& \widetilde{A}_{11}^{(1)} x_{1}+\widetilde{B}_{11}^{(1)} u_{1}=F_{1}^{T} \tilde{\theta}_{1}^{(1)}, \\
& \widetilde{A}_{22}^{(1)} x_{2}+\widetilde{B}_{22}^{(1)} u_{2}=F_{2}^{T} \tilde{\theta}_{2}^{(1)},
\end{aligned}
$$

where $F_{1}^{T}(t)=\left[x_{11} I_{4}, \ldots, x_{14} I_{4}, u_{1} I_{4}\right]$ with $x_{1 i}$ being the $i$ th element of $x_{1}$ and $F_{2}^{T}(t)=$ $\left[x_{21} I_{5}, \ldots, x_{25} I_{5}, u_{2} I_{5}\right]$ with $x_{2 i}$ being the $i$ th element of $x_{2}$. For simplicity, we set $\Gamma_{1}=\Gamma_{3}$ and $\Gamma_{2}=\Gamma_{4}$ for the adaptive laws (11.3.17) and (11.3.18). Then, the adaptive laws (11.3.17) and (11.3.18) can be expressed as

$$
\dot{\tilde{\theta}}_{1}^{(1)}=-\Gamma_{1} P_{1} F_{1}(t) e_{m 1}^{(1)}, \quad \dot{\tilde{\theta}}_{2}^{(1)}=-\Gamma_{2} P_{2} F_{2}(t) e_{m 2}^{(1)}
$$

From (11.3.24), (11.3.25), (11.3.26) and (11.3.27), we have

$$
\begin{aligned}
& {\left[\begin{array}{c}
\dot{e}_{m 1}^{(1)} \\
\dot{\tilde{\theta}}_{1}^{(1)}
\end{array}\right]=A_{01}\left[\begin{array}{c}
e_{m 1}^{(1)} \\
\tilde{\theta}_{1}^{(1)}
\end{array}\right]+\left[\begin{array}{c}
\tilde{f}_{01}^{(1)}-A_{d 2} x_{2}-B_{d 2} u_{2} \\
0
\end{array}\right],} \\
& {\left[\begin{array}{c}
\dot{e}_{m 2}^{(1)} \\
\dot{\tilde{\theta}}_{2}^{(1)}
\end{array}\right]=A_{02}\left[\begin{array}{c}
e_{m 2}^{(1)} \\
\tilde{\theta}_{2}^{(1)}
\end{array}\right]+\left[\begin{array}{c}
\tilde{f}_{02}-A_{d 3} x_{1}-B_{d 3} u_{1} \\
0
\end{array}\right] .}
\end{aligned}
$$

where

$$
A_{01}=\left[\begin{array}{cc}
A_{m 1} & F_{1}^{T} \\
-\Gamma_{1} P_{1} F_{1} & 0
\end{array}\right], \quad A_{02}=\left[\begin{array}{cc}
A_{m 2} & F_{2}^{T} \\
-\Gamma_{2} P_{2} F_{2} & 0
\end{array}\right] .
$$

Consider a positive definite function

$$
V_{1}=e_{m 1}^{(1) T} P_{1} e_{m 1}^{(1)}+\sum_{i=1}^{4} \tilde{a}_{1 i}^{(1) T} \Gamma_{1}^{-1} \tilde{a}_{1 i}^{(1)}+\widetilde{B}_{11}^{(1) T} \Gamma_{1}^{-1} \widetilde{B}_{11}^{(1)}=z_{1}^{T} P_{01} z_{1},
$$


where $z_{1}^{T}=\left[e_{m 1}^{(1) T}, \tilde{\theta}_{1}^{(1) T}\right]$ and $P_{0}=\operatorname{diag}\left\{P_{1}, \Gamma_{1}^{-1}, \ldots, \Gamma_{1}^{-1}\right\}$. From the time derivative of $V_{1}$ along with the adaptive laws in (11.3.17) and (11.3.18), we can have

$$
A_{01}^{T} P_{01}+P_{01} A_{01}+\nu_{1} C_{01} C_{01}^{T} \leq 0
$$

where $\nu_{1}=\lambda_{\min }\left[Q_{1}\right]$ and $C_{0}^{T}=\left[I_{4}, 0\right]$. Since $u_{i}(t), i=1,2$ is sufficiently rich of order $n+1$ and $(A, B)$ is controllable, it follows that $\varphi(t)=\left[x^{T}, u^{T}\right]^{T}$ is $\mathrm{PE}[44]$. Then, we have $\varphi_{1}(t)=\left[x_{1}^{T}, u_{1}\right]^{T}$ is PE [44, Lemma 4.8.3], which together with (11.3.31) results in closed-loop exponential stability [44, Lemma 5.6.3] of the system $\dot{z}_{1}=A_{01} z_{1}$. Since the system (11.3.28) has input signals, we have that $e_{m 1}^{(1)}(t)$ does not converge to 0 . Similarly, we obtain that $e_{m 2}^{(1)}(t)$ does not converge to 0 .

From the above proposition, the residual $e_{m}^{(1)}$ does not converge to zero after damage occurs. However, there may exist other possible situations to prevent $e_{m}^{(1)}$ converging to zero such as some disturbance to the aircraft system. Hence, the condition that $e_{m}^{(1)}$ does not converge to zero may not be enough to detect damage. We will build another detector to work with detector for healthy aircraft system to identify the damaged system.

\subsubsection{Detector for damaged aircraft system}

Here, we build a detector to estimate the coupled system parameters $(A, B)$ after damage occurs:

$$
\dot{x}_{m}^{(2)}(t)=A_{m} x_{m}^{(2)}(t)+\left(\widehat{A}^{(2)}(t)-A_{m}\right) x(t)+\widehat{B}^{(2)}(t) u(t)+\hat{f}_{0}^{(2)}(t)
$$

where $x(t)$ and $u(t)$ are the state and input signals of the aircraft system (11.2.4) which are bounded, $A_{m}$ is a chosen stable matrix, and $\widehat{A}^{(2)}(t), \widehat{B}^{(2)}(t)$ and $\hat{f}_{0}^{(2)}(t)$ are the estimates of damaged aircraft system parameters $A, B$ and $f_{0}$.

Adaptive laws. The residual is defined as $e_{m}^{(2)}(t)=x_{m}^{(2)}-x(t)$. Then, adaptive 
laws for $\widehat{A}^{(2)}(t), \widehat{B}^{(2)}(t)$, and $\hat{f}_{0}^{(2)}(t)$ are chosen as

$$
\begin{aligned}
& \dot{\widehat{A}}^{(2)}(t)=-\Gamma_{d 1} P e_{m}^{(2)}(t) x^{T}(t), \\
& \dot{\widehat{B}}^{(2)}(t)=-\Gamma_{d 2} P e_{m}^{(2)}(t) u^{T}(t), \\
& \dot{\hat{f}}_{0}^{(2)}(t)=-\Gamma_{d f} P e_{m}^{(2)}(t),
\end{aligned}
$$

where $\Gamma_{d 1}, \Gamma_{d 2}$, and $\Gamma_{d f}$ are the symmetric positive definite matrices, and $P=P^{T}>0$, such that $P A_{m}+A_{m}^{T} P=-Q$ with $Q=Q^{T}>0$.

With the adaptive laws (11.3.33)-(11.3.35) and the bounded signals $x(t)$ and $u(t)$ guaranteed by the adaptive control design, the residual $e_{m}^{(2)}(t)$ possess the following property for the healthy or damaged aircraft systems.

Proposition 11.3.3. The residual $e_{m}^{(2)}(t)=x_{m}^{(2)}(t)-x(t)$ between the detector model (11.3.32) updated by (11.3.33)-(11.3.35) and the linearized aircraft system (11.2.4) with the adaptive controller (11.3.1) updated by (11.3.10)-(11.3.12) satisfies that $\lim _{t \rightarrow \infty} e_{m}^{(2)}(t)=0$ for both the healthy and damaged aircraft systems. Moreover, if each element $u_{i}, i=1,2$ of control input $u$ is sufficiently rich of order $n+1$ and uncorrelated, then $\widehat{A}^{(2)}(t)$ and $\widehat{B}^{(2)}(t)$ converge to the system parameters $A$ and $B$ exponentially fast for both the healthy and damaged systems.

Proof: Consider a positive definite function given as

$$
V=e^{(2) T} P e^{(2)}+\operatorname{tr}\left[\widetilde{A}^{(2) T} \Gamma_{d 1}^{-1} \widetilde{A}^{(2)}\right]+\operatorname{tr}\left[\widetilde{B}^{(2) T} \Gamma_{d 2}^{-1} \widetilde{B}^{(2)}\right]+\tilde{f}_{0}^{(2) T} \Gamma_{n f}^{-1} \tilde{f}_{0}^{(2)}
$$

where $\widetilde{A}^{(2)}=\widehat{A}^{(2)}-A_{d}, \widetilde{B}^{(2)}=\widehat{B}^{(2)}-B_{d}$, and $\tilde{f}_{0}^{(2)}=\hat{f}_{0}^{(2)}-f_{0 d}$ for the damaged system, or $\widetilde{A}^{(2)}=\widehat{A}^{(2)}-A_{n}, \widetilde{B}^{(2)}=\widehat{B}^{(2)}-B_{n}, \tilde{f}_{0}^{(2)}=\hat{f}_{0}^{(2)}-f_{0 n}$, for the healthy system. For both the healthy and damaged systems, the residual dynamic equation can be expressed as

$$
\dot{e}^{(2)}(t)=A_{m} e^{(2)}(t)+\widetilde{A}^{(2)}(t) x(t)+\widetilde{B}^{(2)}(t) u(t)+\tilde{f}_{0}^{(2)}(t) .
$$


From (11.3.37), (11.3.36), and the adaptive laws (11.3.33)-(11.3.35), we obtain the time-derivative of $V$ as

$$
\dot{V}=-e^{(2) T}(t) Q e^{(2)}(t) \leq 0
$$

It follows that $e^{(2)}(t) \in L^{2} \cap L^{\infty}$ and $\dot{e}^{(2)} \in L^{\infty}$, along with the bounded $x(t)$ and $u(t)$. Then, we have $\lim _{t \rightarrow \infty} e^{(2)}(t)=0$ for both the healthy and damaged systems.

If each element $u_{i}, i=1,2$ of control input $u$ is sufficiently rich of order $n+1$ and uncorrelated, following the proof of Theorem 5.2.3 in [44], we can conclude that $\widehat{A}^{(2)}(t)$ and $\widehat{B}^{(2)}(t)$ converge to the parameters $A$ and $B$ exponentially fast for the healthy and damaged systems.

From Proposition 11.3.3, we can see that the residual $e_{m}^{(2)}$ from the damaged detector (11.3.32) converge to zero for both the healthy and damaged cases, if there is no other disturbance in the system. Hence, when the residual $e_{m}^{(2)}$ does not converge to zero, it may indicate that there is disturbance. Since the disturbance may also prevent the residual $e_{m}^{(1)}$ from converging to zero, only using $e_{m}^{(1)}$ not converging to zero as a detection criterion may introduce false alarms. Therefore, we need to check the response of $e_{m}^{(2)}$ to prevent false alarms. Moreover, if the control input signal $u(t)$ is rich enough, the damaged detector (11.3.32) can give a good estimate of the damaged aircraft system parameters, which can be used for fault-tolerant feedback control designs.

\subsubsection{Determination of damage status}

From the steady state response of residuals $e_{m}^{(1)}$ and $e_{m}^{(2)}$ as concluded in the above propositions, we obtain the following detection criteria:

- if both $e_{m}^{(1)}$ and $e_{m}^{(2)}$ converge to zero, then there is no damage;

- if $e_{m}^{(1)}$ does not converge to zero, but $e_{m}^{(2)}$ converges to zero, then damage occurs. 
Since disturbance in the aircraft system may prevent the residuals $e_{m}^{(1)}$ and $e_{m}^{(2)}$ converging to zero, in the damage detection criteria, we need to check the response of $e_{m}^{(2)}$ to avoid false alarms.

\subsection{Application to the GTM}

In this section, we will use the GTM to assess the performance of the developed feedback-based damage detection scheme.

Damage scenarios. The GTM Simulink model provides some structural damage scenarios such as rudder off, left outboard flap off, left wing-tip off, left elevator off, and left stabilizer off. In this study, we choose the damage case as the loss of outboard left wing-tip, which is approximate $25 \%$ semi-span of the left wing.

\subsubsection{Simulation Study for the Linearized GTM}

We linearize the healthy and damaged GTMs (11.1.1) at an operating condition (wings-level flight at 100 knots) $\left(x_{0}, u_{0}\right)$. Then, the linearized GTM is given as (11.2.1): $\Delta \dot{x}=A \Delta x+B \Delta u+f_{0}, \Delta y=C \Delta x$. We obtain the numerical values for the system parameters before and after the loss of wing-tip damage occurs.

Verification of design conditions. The numerical values are only used for building the simulation model, but not used for the detection scheme design. From the numerical values, we can see that the longitudinal and lateral-directional dynamics are decoupled before damage occurs and coupled after damage occurs. The invariant properties of infinity zero structure and signs of leading principal minors have been shown by the generic linearized models. Here, we further verify the design conditions by using the numerical values. We can calculate the interactor matrix as $\xi_{m}(s)=$ $\operatorname{diag}\left\{(s+1)^{2},(s+1)^{2}\right\}$ for both healthy and damaged GTMs. Then, we have the high 
frequency gain matrix for the healthy GTM is

$$
K_{p}=\lim _{s \rightarrow \infty} \xi_{m}(s) G(s)=\left[\begin{array}{cc}
-1.348 & 0 \\
0 & -0.737
\end{array}\right]
$$

and the high frequency gain matrix for the damaged GTM is

$$
K_{p}=\lim _{s \rightarrow \infty} \xi_{m}(s) G(s)=\left[\begin{array}{cc}
-1.327 & -0.0027 \\
0.0069 & -0.7576
\end{array}\right] .
$$

Therefore, the leading principal minors are invariant before and after damage, where

$$
\operatorname{sign}\left(\Delta_{1}\right)=-1, \quad \operatorname{sign}\left(\Delta_{2}\right)=1
$$

Adaptive feedback control. The reference model is chosen as $\Delta y_{m}(t)=$ $W_{m}(s)[r](t)$, where $W_{m}(s)=\xi_{m}^{-1}(s)=\operatorname{diag}\left\{1 /(s+1)^{2}, 1 /(s+1)^{2}\right\}$. We apply $\Delta u(t)=K_{1}^{T}(t) \Delta x(t)+K_{2}(t) r(t)+k_{3}(t)$ with the adaptive laws (11.3.10)-(11.3.12) to the linearized model (11.2.1) to ensure the signal boundedness and output tracking.

Detector models. We run the detector (11.3.13):

$$
\Delta \dot{x}_{m}^{(1)}(t)=A_{m} x_{m}^{(1)}(t)+\left(\widehat{A}^{(1)}(t)-A_{m}\right) \Delta x(t)+\widehat{B}^{(1)}(t) \Delta u(t)+\hat{f}_{0}^{(1)}(t)
$$

with the adaptive laws (11.3.17)-(11.3.19) and the model (11.3.32):

$$
\Delta \dot{x}_{m}^{(2)}(t)=A_{m} x_{m}^{(2)}(t)+\left(\widehat{A}^{(2)}(t)-A_{m}\right) \Delta x(t)+\widehat{B}^{(2)}(t) \Delta u(t)+\hat{f}_{0}^{(2)}(t)
$$

with the adaptive laws (11.3.33)-(11.3.35) in parallel to obtain the detector state signals $\Delta x_{m}^{(1)}(t)$ and $\Delta x_{m}^{(2)}(t)$ for the linearized simulation. Then, we can have the residuals as $\Delta e_{m}^{(1)}=\Delta x_{m}^{(1)}-\Delta x$ and $\Delta e_{m}^{(2)}=\Delta x_{m}^{(2)}-\Delta x$. It is worth noting that the signals $\Delta x$ and $\Delta u$ are the state and control signals of the linearized aircraft system.

Simulation results. The output response and residual response are shown in Fig. 11.1 and Fig. 11.2, where the reference input signal $r(t)$ is rich of frequencies. The damage (loss of wing-tip) occurs at 100 seconds. From Fig. 11.1, we can see that 
the output signal tracks the reference signal before and after damage occurs. The response of the 8 th elements of $\Delta e_{m}^{(1)}$ and $\Delta e_{m}^{(2)}$ (i.e. errors between roll angle $\Delta \phi$ whose unit is degree and its estimates) are illustrated in Fig. 11.2. From Fig. 11.2, we have that both residual $\Delta e_{m}^{(1)}$ and $\Delta e_{m}^{(2)}$ converge to zero before damage occurs. Then, after some transient response starting at 100 seconds, $\Delta e_{m}^{(1)}$ fails to converge to zero, while $\Delta e_{m}^{(2)}$ converges to zero. Therefore, we conclude that damage occurs at 100 seconds.
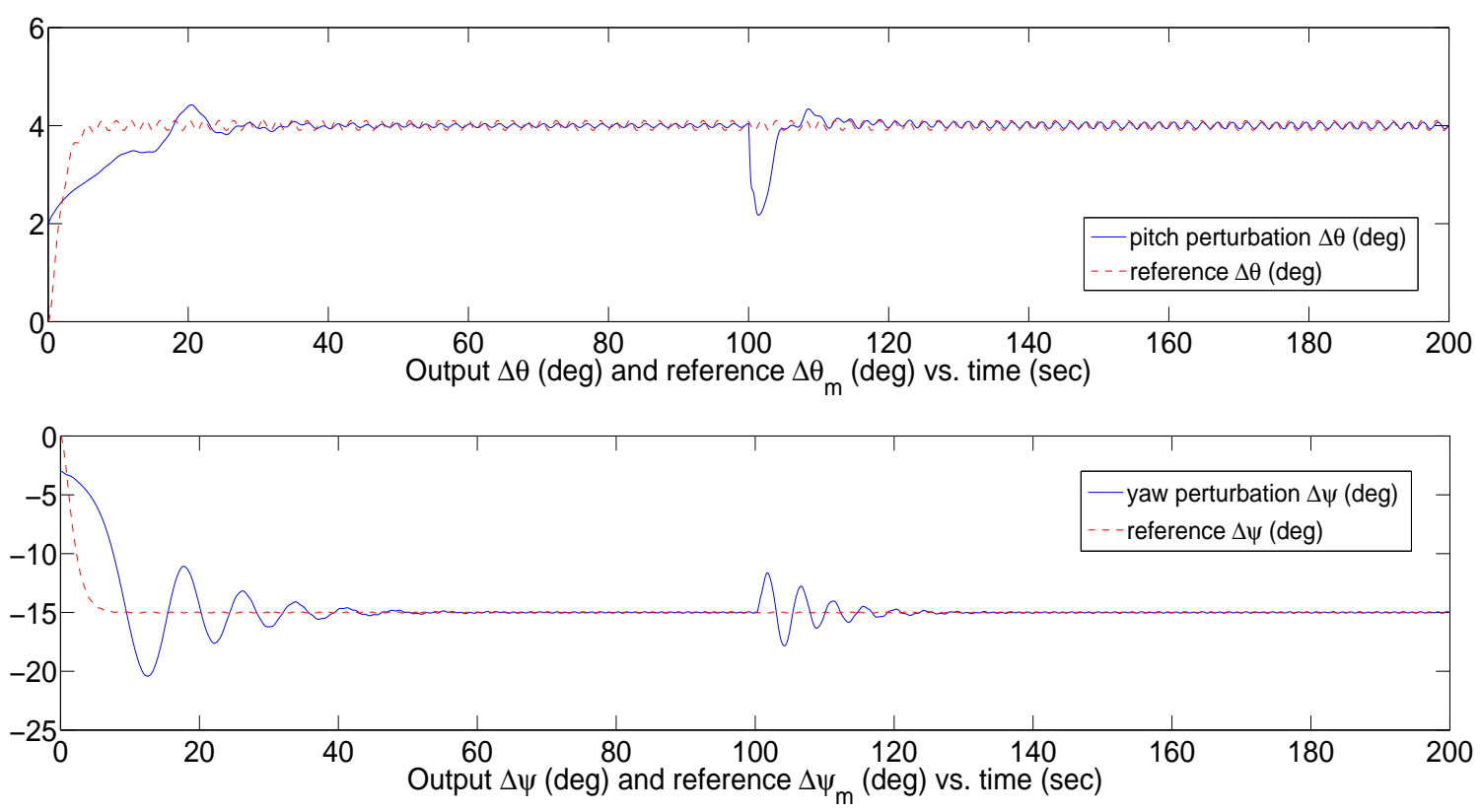

Figure 11.1: Linearized GTM outputs (solid) vs. reference outputs (dotted).

\subsubsection{Simulation Study for the Nonlinear GTM}

Since the simulation study for the linearized GTM has verified the proposed design, we will apply it to the original nonlinear GTM to assess the effectiveness of this linearization-based design. For the nonlinear GTM simulation study, we will investigate three cases: Case I is that GTM is operated around an operating point $\left(x_{0}, u_{0}\right)$ 

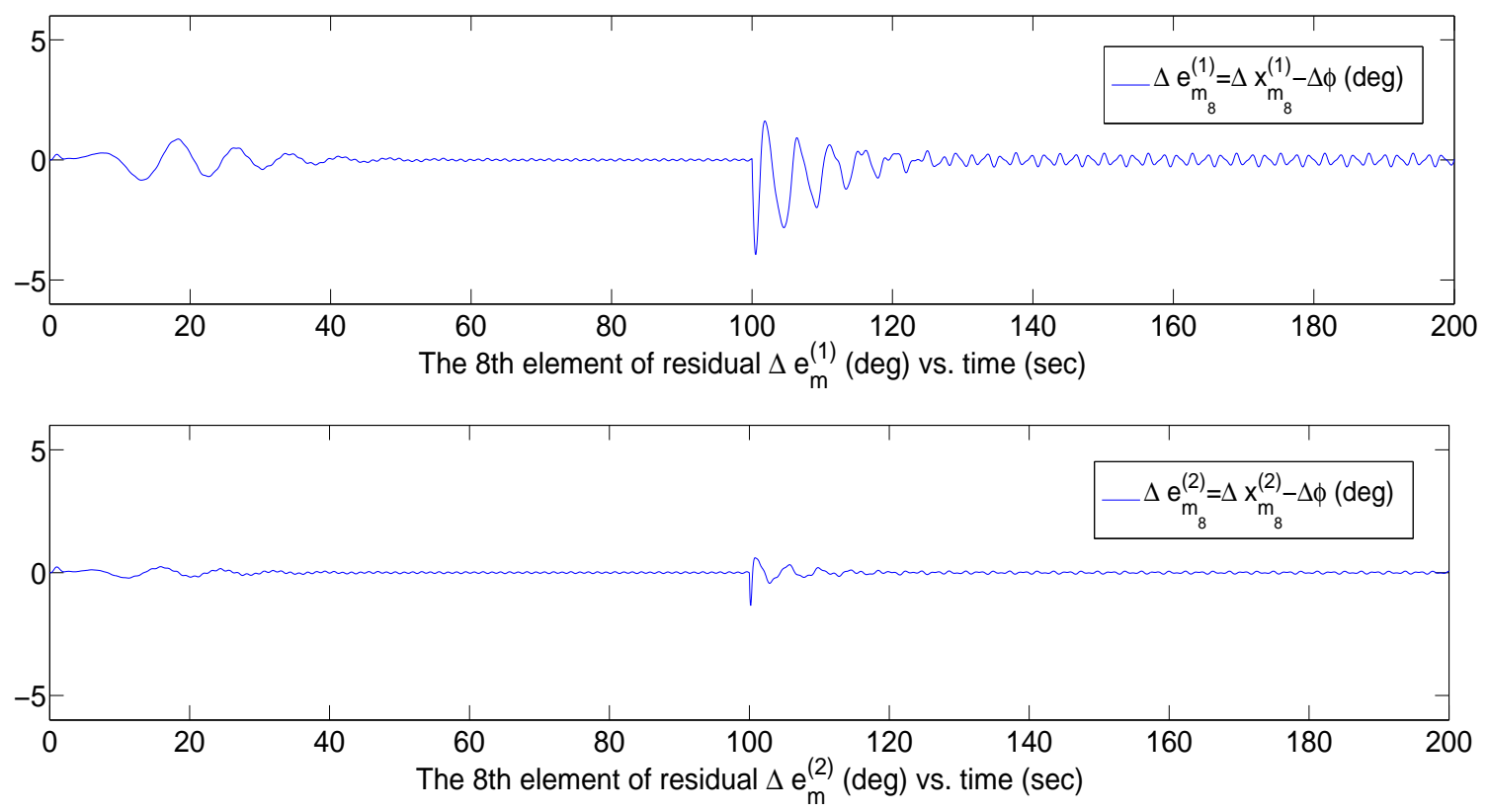

Figure 11.2: Detector residuals $\Delta e_{m 8}^{(1)}$ and $\Delta e_{m 8}^{(2)}$.

obtained by trimming the healthy GTM at a wing-level flight condition with equivalent airspeed as 100 knots; Case II is that GTM is operated around another operating point $\left(x_{0}, u_{0}\right)$ obtained by trimming the GTM at a wing-level flight condition with equivalent airspeed as 90 knots; Case III is that GTM is operated from the second case operating point to the first case operating point. For all these three cases, the wing-tip off damage can occur at any moments.

We apply the control law $u(t)=\Delta u(t)+u_{0}$ to the nonlinear GTM around a small neighborhood of $\left(x_{0}, u_{0}\right)$. Then, we construct the detectors (11.4.1) and (11.4.2) by using the signals $\Delta x(t)$ and $\Delta u(t)$. It is worth noting that the signals $\Delta x(t)$ and $\Delta u(t)$ are calculated from the nonlinear GTM state signal $x(t)$ and control input signal $u(t)$, i.e. $\Delta x(t)=x(t)-x_{0}$ and $\Delta u(t)=u(t)-u_{0}$. The simulation results are given as follows.

Case I: around $\left(x_{0}, u_{0}\right)$ with airspeed as 100 knots. Fig. 11.3 and Fig. 11.4 show 
the response of the GTM state and control input signals, where we can see that the signals are bounded and the output signals track the reference signals. From the response of the 8th elements of $\Delta e_{m}^{(1)}$ and $\Delta e_{m}^{(2)}$ (i.e. errors between roll angle $\Delta \phi$ and its estimates) in Fig. 11.5, where both residuals converge to zero before 80 seconds and $\Delta e_{m}^{(1)}$ does not converge to zero while $\Delta e_{m}^{(2)}$ converges to zero, we can conclude that the damage happens at 80 seconds.
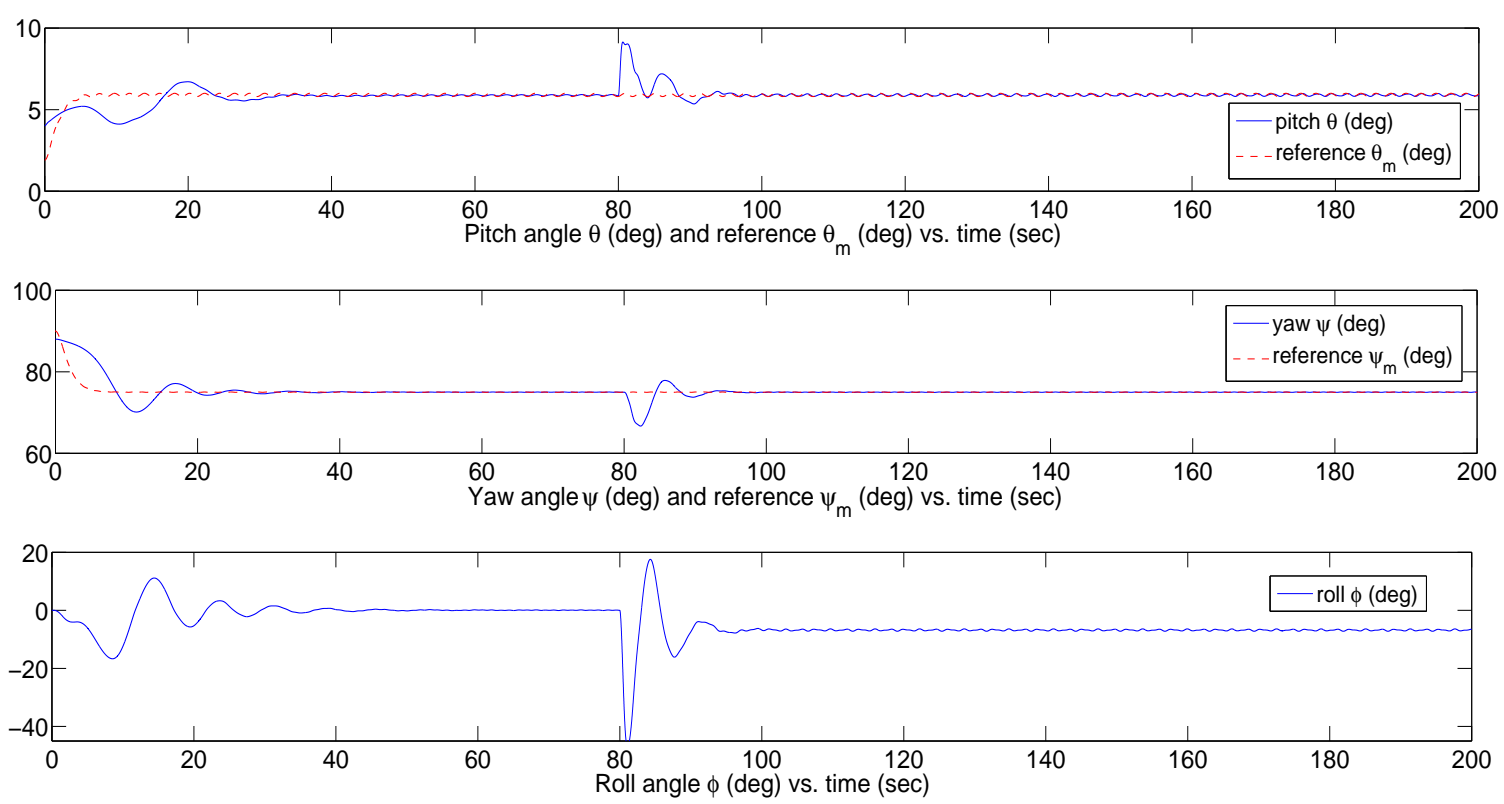

Figure 11.3: GTM responses: pitch $\theta(t)$, yaw $\psi(t)$, and roll $\phi(t)$ (case I).

Case II: around $\left(x_{0}, u_{0}\right)$ with airspeed as 90 knots. From Fig. 11.6 and Fig. 11.7, we can see that the signals are bounded and the output signals track the reference signals around a small neighborhood of the given operating point. Then, from the response of the 8 th elements of $\Delta e_{m}^{(1)}$ and $\Delta e_{m}^{(2)}$ shown in Fig. 11.8, where both residuals converge to zero before 120 seconds and $\Delta e_{m}^{(1)}$ does not converge to zero while $\Delta e_{m}^{(2)}$ converges to zero after 120 seconds, we conclude that the damage happens at 120 seconds. 

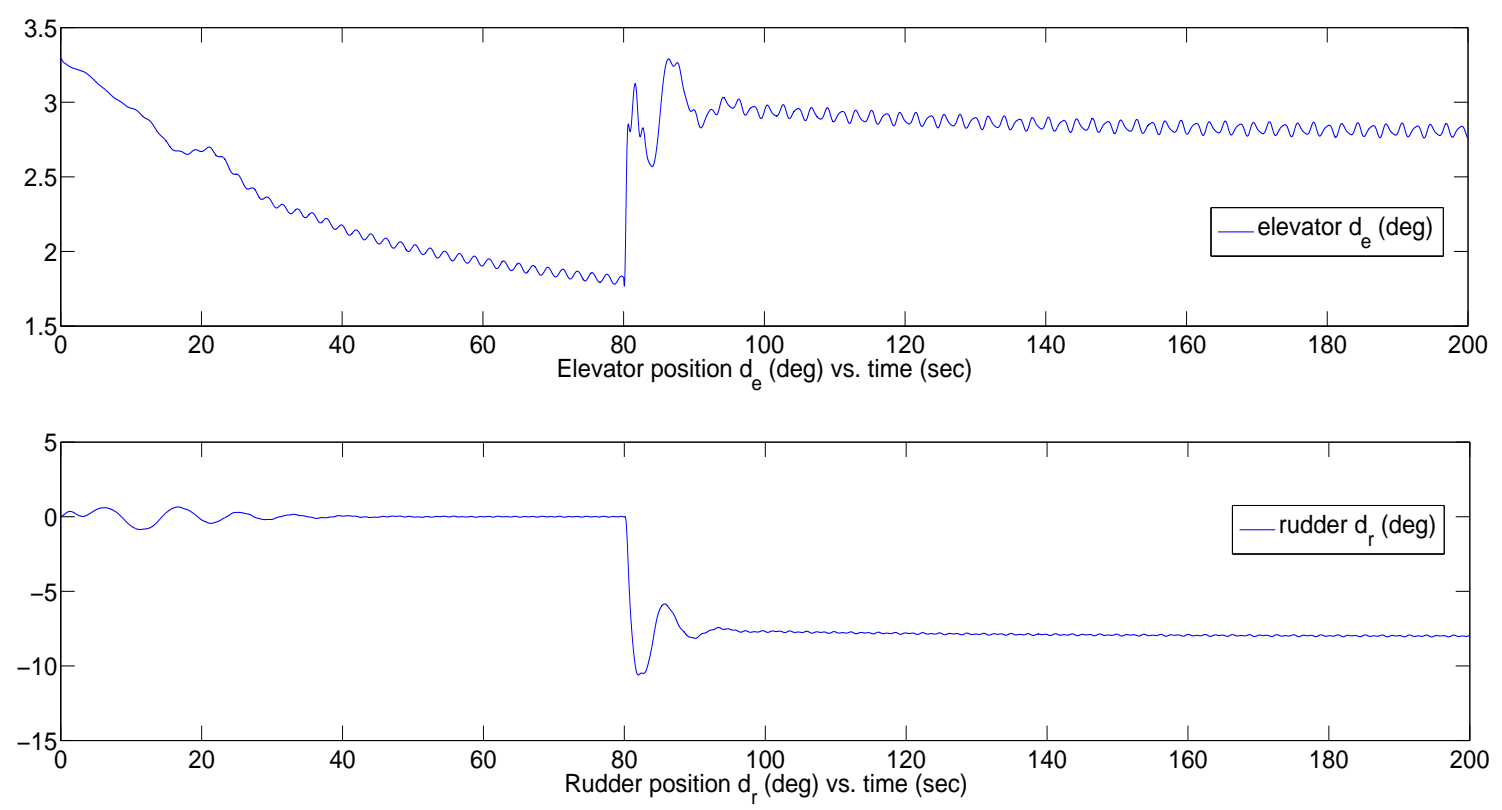

Figure 11.4: Control surface positions: elevator $d_{e}(t)$ and rudder $d_{r}(t)$ (case I).
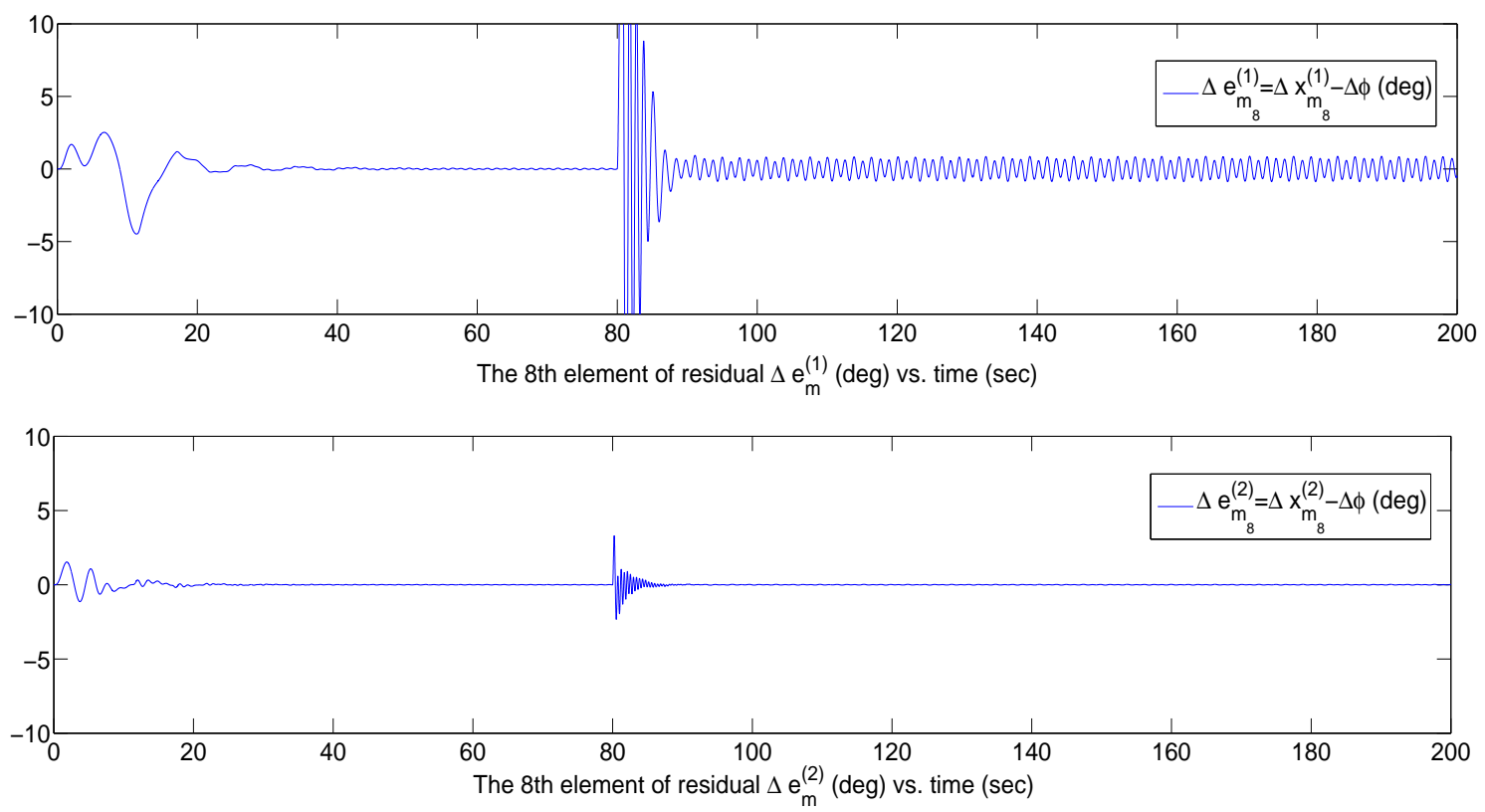

Figure 11.5: Detector residuals $\Delta e_{m 8}^{(1)}$ and $\Delta e_{m 8}^{(2)}$ (case I). 

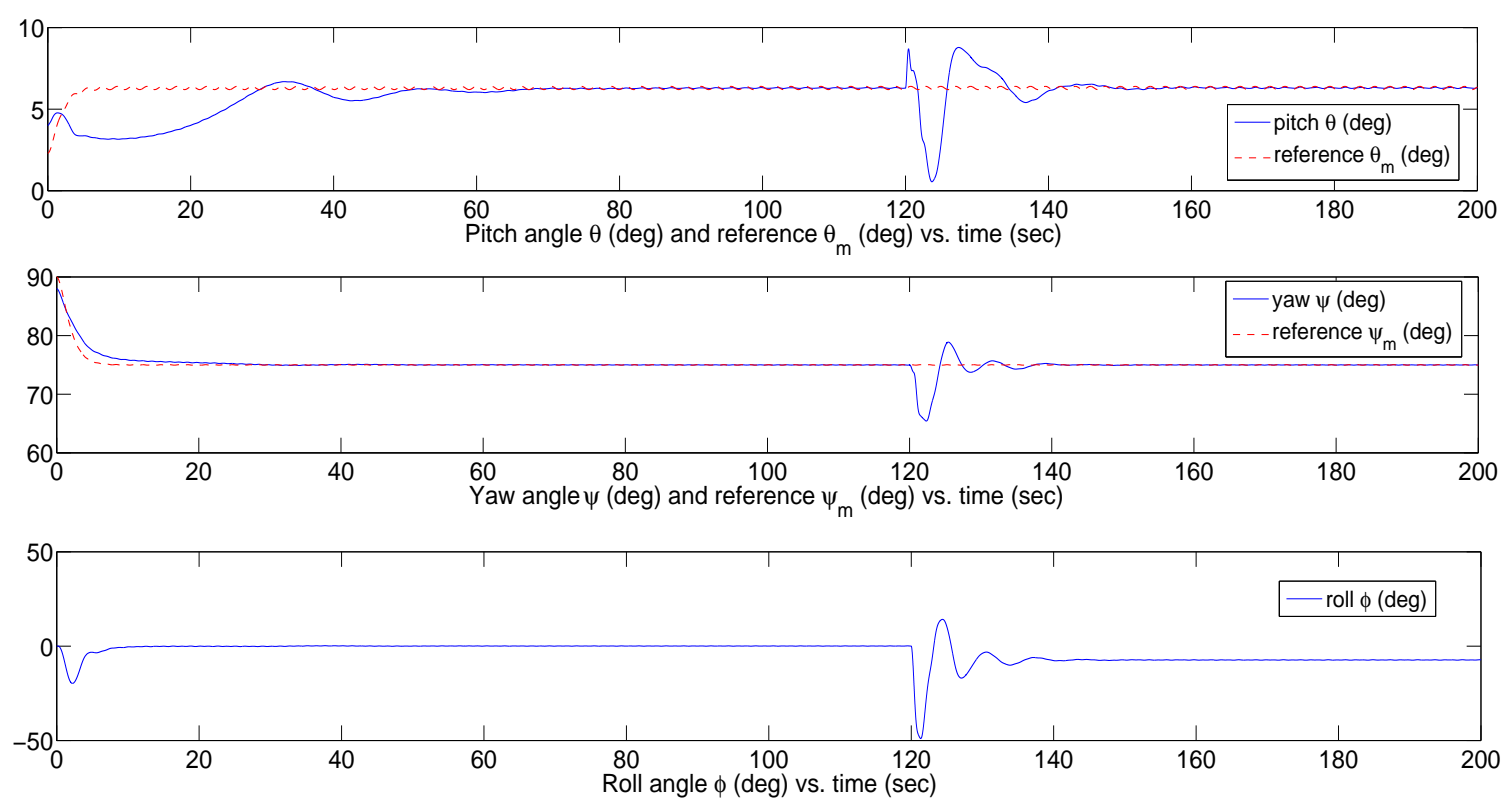

Figure 11.6: GTM responses: pitch $\theta(t)$, yaw $\psi(t)$, and roll $\phi(t)$ (case II).
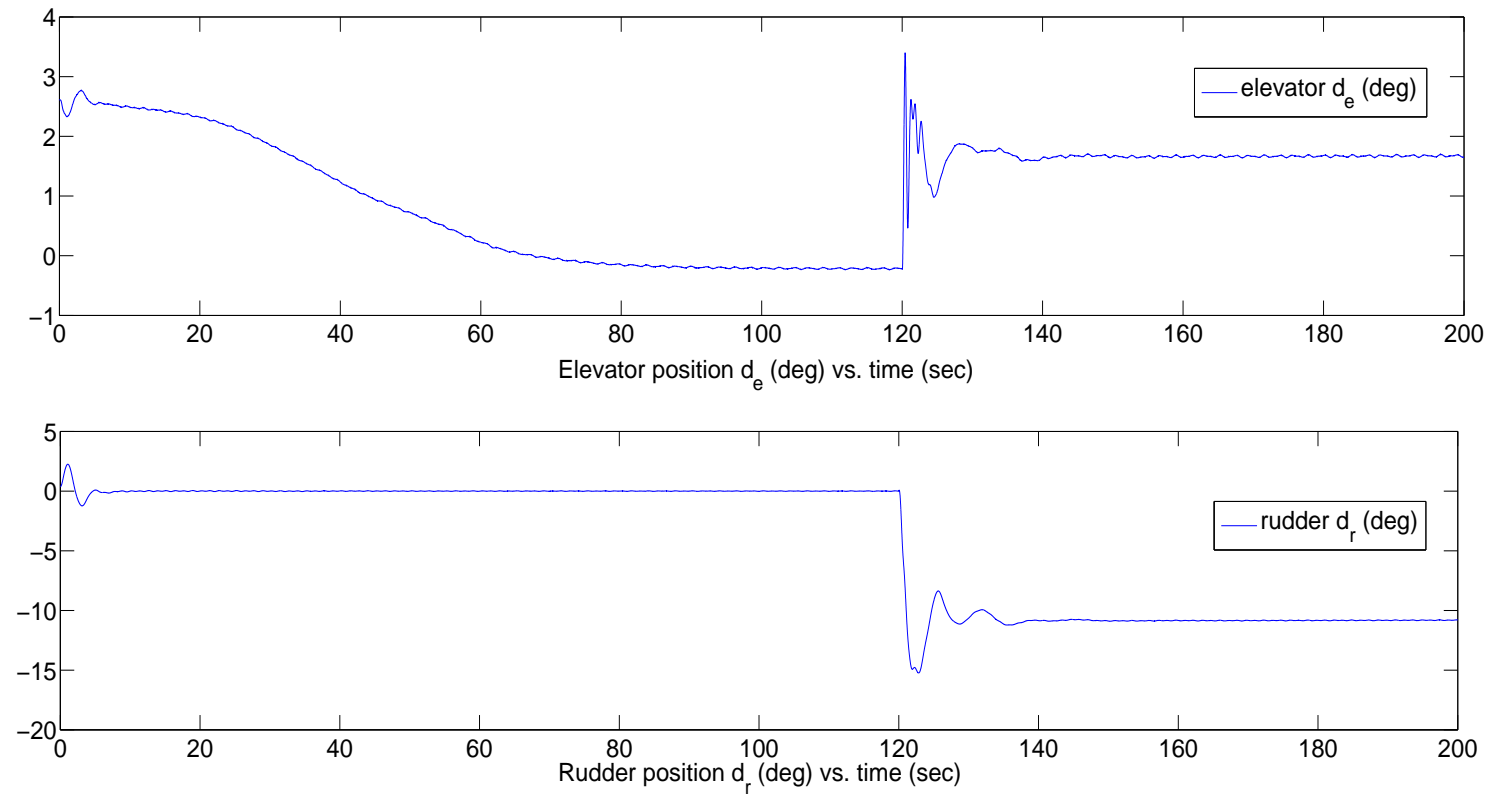

Figure 11.7: Control surface positions: elevator $d_{e}(t)$ and rudder $d_{r}(t)$ (case II). 

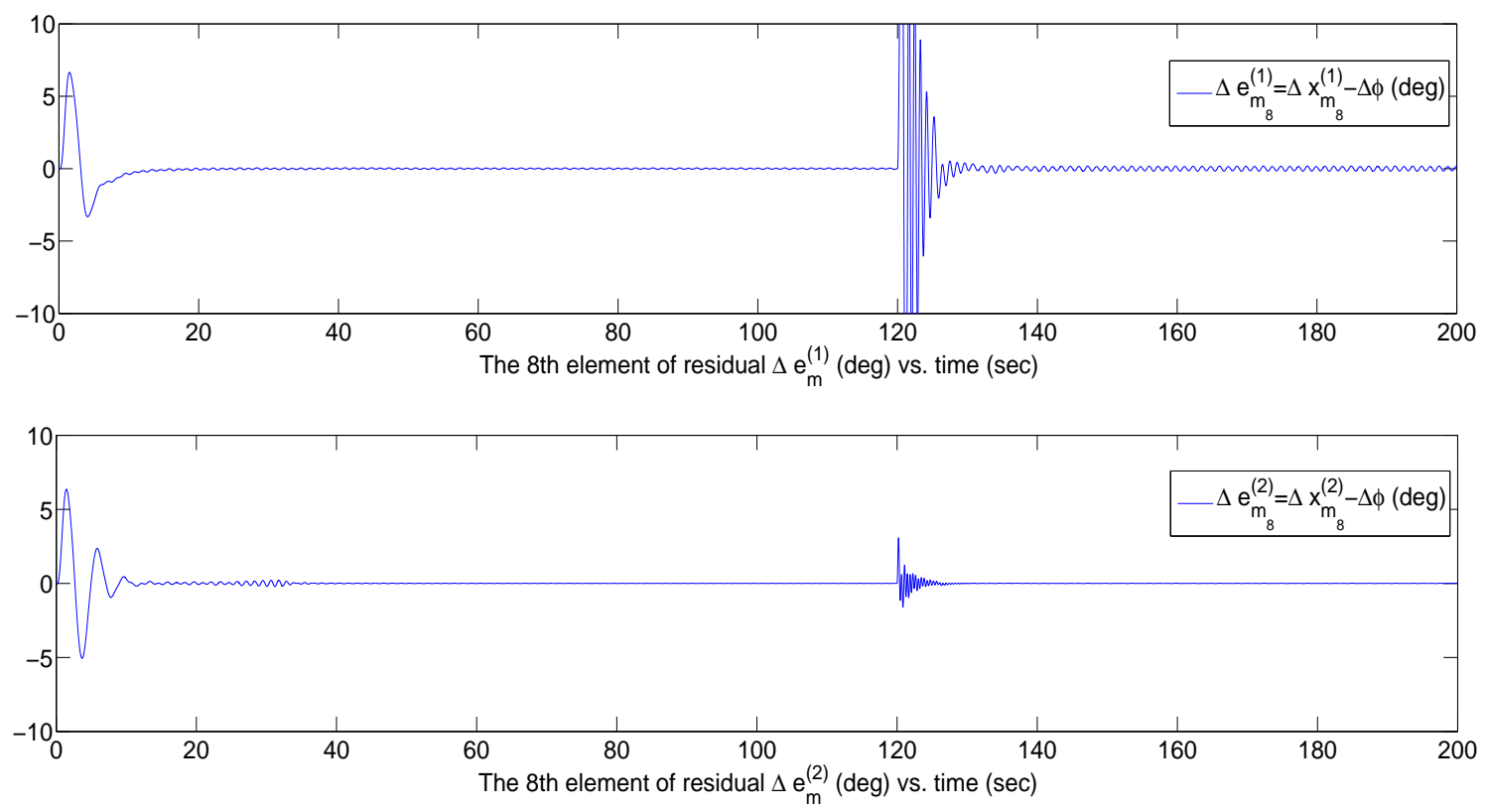

Figure 11.8: Detector residuals $\Delta e_{m 8}^{(1)}$ and $\Delta e_{m 8}^{(2)}$ (case II).

Case III: around the neighborhood of $\left(x_{0}, u_{0}\right)$ with airspeed as 90 knots to the neighborhood of $\left(x_{0}, u_{0}\right)$ with airspeed as 100 knots. In this simulation, we change the operating condition at 120 seconds. Fig. 11.9 and Fig. 11.10 show the response of the GTM state and control input signals, where we can see that the signals are bounded and the output signals track the reference signals. Fig. 11.11 illustrates the detectors response. Although there is some transient response of the GTM signals in Fig. 11.9 when we change the operating condition at 120 seconds, from Fig. 11.11, we can see that both the residuals converge to zero before and after 120 seconds, which means that there are no damage occurring. But after 180 seconds, $\Delta e_{m}^{(1)}$ does not converge to zero while $\Delta e_{m}^{(2)}$ converges to zero, where we conclude that the damage happens at 180 seconds. It is worth noting that when we change operating conditions or damage occurs, there can be some transient response of the GTM state signals, so that we need the damage detection scheme to enhance the awareness of flight situations for 
the flight control personnel.
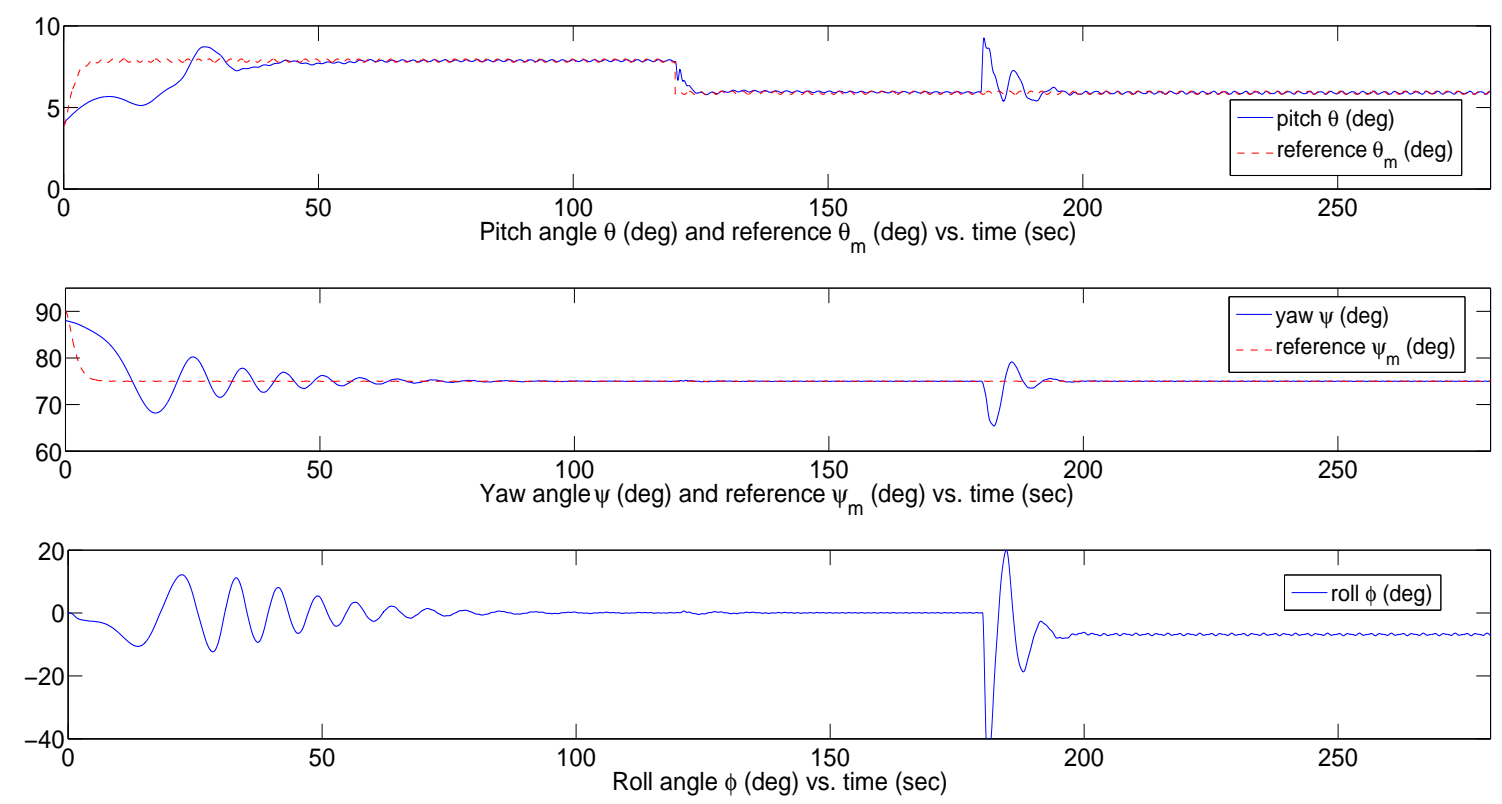

Figure 11.9: GTM responses: pitch $\theta(t)$, yaw $\psi(t)$, and roll $\phi(t)$ (case III).

\section{Summary}

In this chapter, the self-stabilization based damage detection scheme have been developed for the aircraft systems. When damage happens, the longitudinal and lateraldirectional dynamics are coupled. To capture such a feature, two detection models have been established to estimate the decoupled and coupled parameters for the healthy and damaged cases. To ensure the signal boundedness, the multivariable state feedback for output tracking MRAC scheme has been applied to the aircraft systems with damage. Simulation studies of the linearized and nonlinear GTM have been conducted to show the performance of the proposed damage detection scheme and the effectiveness of this linearization-based damage detection design. 

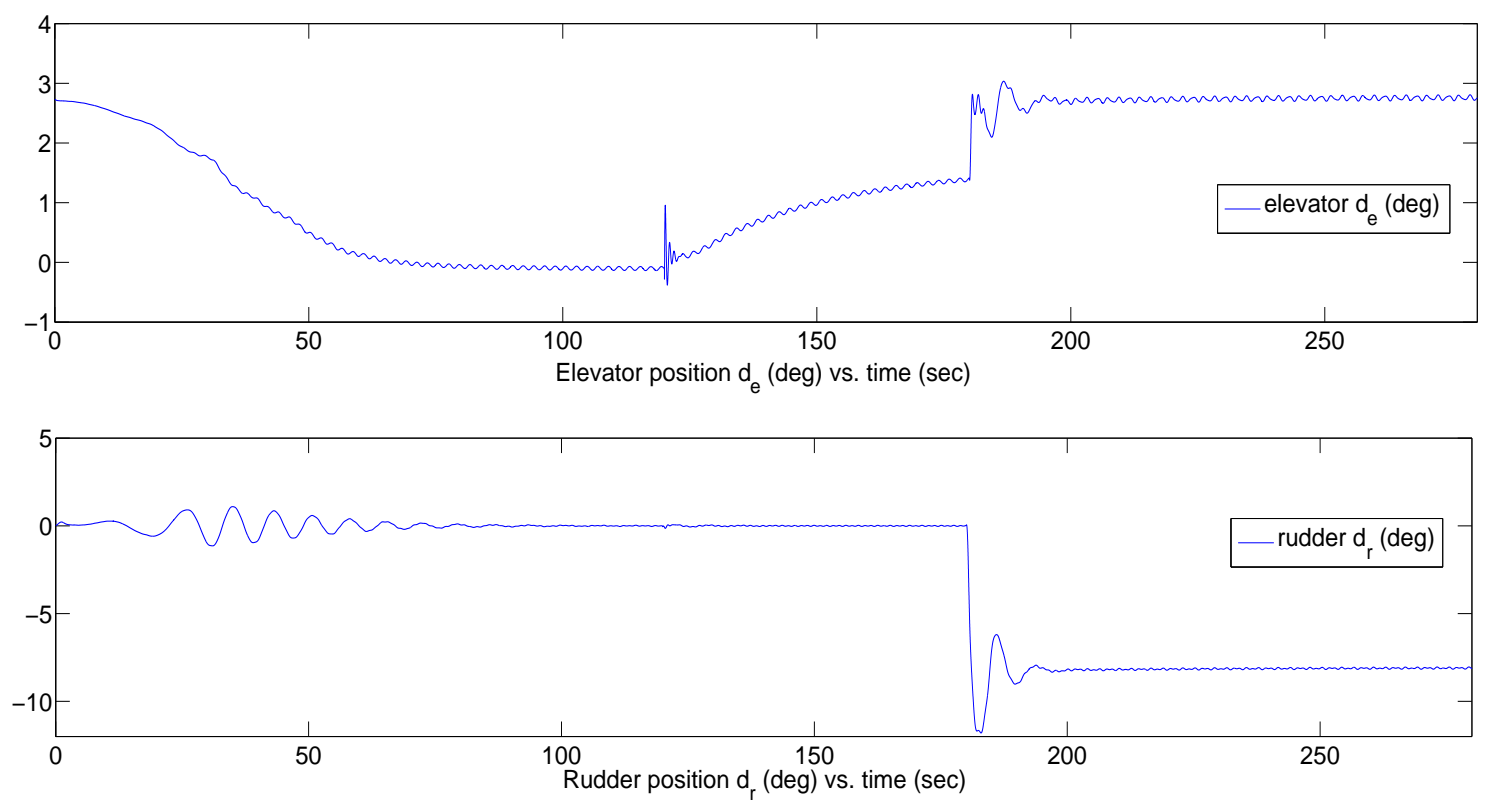

Figure 11.10: Control surface positions: elevator $d_{e}(t)$ and rudder $d_{r}(t)$ (case III).
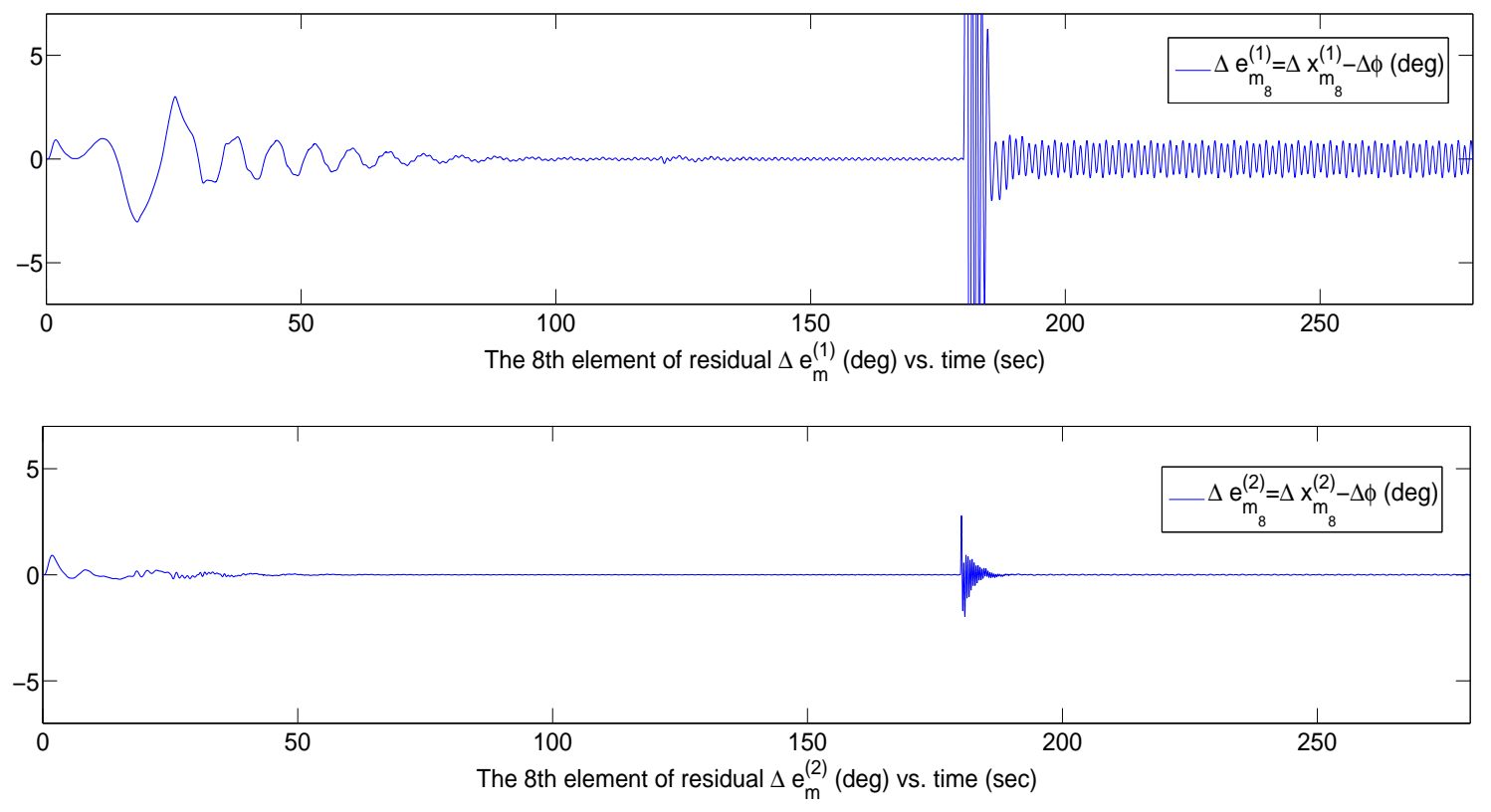

Figure 11.11: Detector residuals $\Delta e_{m 8}^{(1)}$ and $\Delta e_{m 8}^{(2)}$ (case III). 


\section{Chapter 12}

\section{Feedback-Based Adaptive Sensor Uncertainty Detection}

In this chapter, an adaptive feedback-based stable fault detection scheme is developed for linear time-invairant systems with sensor uncertainties and system parameter uncertainties. A parametric sensor uncertainty model with additive faults and multiplicative faults is introduced. To employ the model-based fault detection method, the sensor dynamics is derived. Based on the newly developed sensor dynamic models, a set of estimation model systems are established to estimate the sensor signals. Unlike most fault detection schemes which operate under the assumption that all system signals remain bounded under sensor uncertainty conditions, the adaptive sensor detection scheme proposed in this chapter is integrated with an adaptive feedback controller which is designed to ensure the desired signal boundedness requirement needed for stable sensor uncertainty detection operation. By observing residuals between the sensor signals and the estimation model signals, the sensor uncertainty can be detected and specific uncertainty patterns can be identified. Desired adaptive sensor uncertainty detection performance is demonstrated in the simulation study for a linearized longitudinal aircraft flight control system. 


\subsection{Problem Statement}

Consider a single-input and single-output (SISO) linear time-invariant system

$$
\dot{x}(t)=A x(t)+B u(t), \quad y(t)=C x(t),
$$

where $A \in R^{n \times n}, B \in R^{n \times 1}$, and $C \in R^{1 \times n}$, are unknown constant parameter matrices, and $x(t) \in R^{n}, u(t) \in R$, and $y(t) \in R$ are system state, input, and output signals. For feedback control designs, construction of the input signal $u(t)$ is based on sensor measurements of the state signal $x(t)$ or the output signal $y(t)$. Performance of the feedback control system can be deteriorated when there are uncertainties in the sensor measurements.

Sensor uncertainty model. For the detection and compensation designs, a sensor uncertainty model is given as

$$
z(t)=k_{s} \varphi(t)+\sum_{i=1}^{q} b_{i} f_{i}(t)
$$

where $\varphi(t)$ is the actual signal to be measured, which can be the state $x(t)$ or output $y(t), k_{s}>0$ and $b_{i}, i=1,2, \ldots, q$, are some unknown constant sensor uncertainty parameters, and $f_{i}(t), i=1,2, \ldots, q$, are known bounded signals with bounded derivative $\dot{f}_{i}(t)$.

Remark 12.1.1. The sensor uncertainty modeling problem can be addressed by using redundant sensors. We can use several sensors to measure the same signal $\varphi(t)$, and take the weighted sum of the sensors' output signals $z_{i}(t)$ as $z(t)=\sum_{i=1}^{m} \alpha_{i} z_{i}(t)$, where $\alpha_{i}>0, i=1, \ldots, m$, such that $\sum_{i=1}^{m} \alpha_{i}=1$. When there is no uncertainty for all the sensors, the summed sensor signal $z(t)$ is the exact measured signal $\varphi(t)$. When there are some sensor uncertainties, e.g., the $i_{1}, i_{2}, \ldots, i_{p}$ th sensors fail and generate some random signals $\bar{z}_{i}(t)$, the summed sensor signal is $z(t)=\alpha_{s} \varphi(t)+d_{s}(t)$, where 
$\alpha_{s}=\sum_{i \neq i_{1}, i_{2}, \ldots, i_{p}} \alpha_{i}$, and $d_{s}(t)=\sum_{i=i_{1}, i_{2}, \ldots, i_{p}} \alpha_{i} \bar{z}_{i}(t)$. Since the indexes $i_{1}, i_{2}, \ldots, i_{p}$ are unknown, we may express $d_{s}(t)=\sum_{i=1}^{m} \beta_{i} \bar{z}_{i}(t)$, where some of $\beta_{i}$ are zero (for the unfailed sensors) while others are $\alpha_{i}$, and $\bar{z}_{i}$ is accessible in the bias-uncertainty part of the uncertainty model (12.1.2).

Feedback-based uncertainty detection problem. Based on the sensor uncertainty models (12.1.2), we will develop a model-based adaptive diagnosis scheme to detect the modeled sensor uncertainty. More specifically, a set of state sensor detectors and output sensor detectors are designed, which are a set of adaptive estimation models to estimate the unknown parameters in the sensor uncertainty models. By comparing residuals between the detection models and the sensor uncertainty models, detection criteria can be derived to identify particular uncertainty scenarios.

Feedback uncertainty compensation problem. The construction of detection models requires that the control input signal $u(t)$, the state sensor signal $z_{x}(t)$, and the output sensor signal $z_{y}(t)$ are bounded. To ensure the signal boundedness requirement, an adaptive feedback control law will be developed for the system (12.1.1) to compensate the sensor uncertainty and make all the closed-loop signals bounded including the signals $u(t), z_{x}(t)$, and $z_{y}(t)$.

Since the parameters $k_{s}$ and $b_{i}, i=1,2, \ldots, q_{i}$, of the state or output sensor model (12.1.2) are unknown, the state signal $x(t)$ or the output signal $y(t)$ cannot be retrieved from the sensor measurement $z(t)$. To overcome this difficulty, we propose to use sensor compensator signals $\hat{x}(t)$ and $\hat{y}(t)$ from the sensor measurement $z(t)$ to construct an adaptive feedback control law $u(t)$, which can make all the closedloop signals bounded and the plant output signal $y(t)$ track a given reference signal $y_{m}(t) \in R$ generated from a reference model system

$$
y_{m}(t)=W_{m}(s)[r](t)
$$


with $r(t) \in R$ being a bounded reference input signal.

Assumptions. To proceed the control and detection scheme designs, for the system (12.1.1):

$$
y(t)=C(s I-A)^{-1} B[u](t)=\frac{Z(s)}{P(s)}[u](t),
$$

where $Z(s)=z_{m} s^{m}+\cdots+z_{1} s+z_{0}$ with $z_{m} \neq 0$ and $P(s)$ is a monic polynomial of degree $n$, we assume that (A1) $Z(s)$ is a Hurwitz polynomial; (A2) the degree $m$ of $Z(s)$ is known, and $W_{m}(s)=\frac{1}{P_{m}(s)}$ where $P_{m}(s)$ is a Hurwitz polynomial of degree $n-m$; (A3) the sign of $z_{m}$ is known; (A4) $(A, B, C)$ is controllable and observable.

\subsection{Feedback-Based Sensor Uncertainty Detection Scheme}

In this section, we will present the detailed adaptive feedback-based sensor uncertainty detection design. To develop the uncertainty detection scheme, dynamic models with signals being the state sensor $z_{x}(t)$ and the output sensor $z_{y}(t)$ will be given first.

State sensor dynamic model. From the sensor uncertainty model (12.1.2), the state sensor signal $z_{x}(t)$ is expressed as

$$
z_{x}(t)=K_{x} x(t)+\Theta_{b x}^{T} f_{x}(t)
$$

where the unknown parameters $K=\operatorname{diag}\left\{k_{x 1}, \ldots, k_{x n}\right\}, \Theta_{b x}^{T}=\operatorname{diag}\left\{\theta_{b x 1}^{T}, \ldots, \theta_{b x n}^{T}\right\}$ with $\theta_{b x i}=\left[b_{x i 1}, \ldots, b_{x i q_{i}}\right]^{T}$, and the signal $f_{x}(t)=\left[f_{x 1}^{T}(t), \ldots, f_{x n}^{T}(t)\right]^{T}$ with $f_{x i}(t)=$ $\left[f_{x i 1}(t), \ldots, f_{x i q_{i}}(t)\right]^{T}, i=1, \ldots, n$. In view of (12.2.1) and the system (12.1.1), we have the state sensor dynamics as

$$
\dot{z}_{x}(t)=A_{z} z_{x}(t)+B_{z} u(t)+\Theta_{z}^{T} f_{x}(t)+\Theta_{b x}^{T} \dot{f}_{x}(t)
$$

where the unknown parameter matrices are given as

$$
A_{z}=K_{x} A K_{x}^{-1}, B_{z}=K_{x} B, \Theta_{z}^{T}=-K_{x} A K_{x}^{-1} \Theta_{b x}^{T}
$$


Output sensor parametric model. The output sensor with uncertainties is given as

$$
z_{y}(t)=k_{y} y(t)+\theta_{b y}^{T} f_{y}(t)
$$

where $\theta_{b y}=\left[b_{y 1}, \ldots, b_{y p}\right]^{T}$ and $f_{y}(t)=\left[f_{y 1}, \ldots, f_{y p}\right]^{T}$. Operating both sides of the transfer function (12.1.4) by a filter $1 / \Lambda(s)$, where $\Lambda(s)$ is a chosen stable and monic polynomial of degree $n$, we can obtain

$$
y(t)=\frac{Z(s)}{\Lambda(s)}[u](t)+\frac{\Lambda(s)-P(s)}{\Lambda(s)}[y](t) .
$$

Substituting $y(t)=\frac{1}{k_{y}} z_{y}(t)-\frac{\theta_{b y}^{T}}{k_{y}} f_{y}(t)$ in (12.2.5), we have

$$
z_{y}(t)=\theta_{u}^{T} \phi_{u}(t)+\theta_{z}^{T} \phi_{z}(t)+\theta_{b f}^{T} f_{y}(t)+\sum_{i=1}^{p} \theta_{i}^{T} \phi_{i}(t),
$$

where the signals are

$$
\begin{aligned}
\phi_{u}(t) & =\left[\frac{1}{\Lambda(s)}[u](t), \frac{s}{\Lambda(s)}[u](t), \ldots, \frac{s^{m}}{\Lambda(s)}[u](t)\right]^{T}, \\
\phi_{z}(t) & =\left[\frac{1}{\Lambda(s)}\left[z_{y}\right](t), \frac{s}{\Lambda(s)}\left[z_{y}\right](t), \ldots, \frac{s^{(n-1)}}{\Lambda(s)}\left[z_{y}\right](t)\right]^{T}, \\
\phi_{i}(t) & =\left[\frac{1}{\Lambda(s)}\left[f_{y i}\right](t), \frac{s}{\Lambda(s)}\left[f_{y i}\right](t), \ldots, \frac{s^{(n-1)}}{\Lambda(s)}\left[f_{y i}\right](t)\right]^{T},
\end{aligned}
$$

for $i=1, \ldots, p$, and $\theta_{u}, \theta_{z}$, and $\theta_{i}$, are the corresponding unknown parameters.

Signal boundedness. The uncertainty detector models will be developed based on the dynamic models (12.2.2) and (12.2.6) with bounded signals. To ensure the signal boundedness requirement, an adaptive control design will be applied.

\subsubsection{Self-stabilization Feedback Control Design}

Since the state sensors have uncertainties, a state sensor compensator will be used to constructed the controller. 
State sensor compensator. From the state sensor uncertainty model (12.2.1), the state signal $x(t)$ can be retrieved as

$$
x(t)=\left[x_{1}(t), \ldots, x_{n}(t)\right]^{T}=\Theta_{x}^{* T} \psi_{x}(t),
$$

where $\psi_{x}(t)=\left[\psi_{x 1}^{T}, \ldots, \psi_{x n}^{T}\right]^{T}$ with $\psi_{x i}(t)=\left[z_{x i}, f_{x i 1}, \ldots, f_{x i q_{i}}\right]^{T}$ and the unknown parameter $\Theta_{x}^{*}$ is given as $\Theta_{x}^{* T}=\operatorname{diag}\left\{\theta_{x 1}^{* T}, \theta_{x 2}^{* T}, \ldots, \theta_{x n}^{* T}\right\}$ with $\theta_{x i}^{*}, i=1, \ldots, n$ being the corresponding unknown parameters. Since the parameter $\Theta_{x}^{*}$ is unknown, the actual signal $x(t)$ is not accessible. Thus, a sensor compensator is introduced:

$$
\hat{x}(t)=\left[\hat{x}_{1}(t), \ldots, \hat{x}_{n}(t)\right]^{T}=\Theta_{x}^{T}(t) \psi_{x}(t)
$$

where $\Theta_{x}^{T}(t)=\operatorname{diag}\left\{\theta_{x 1}^{T}(t), \theta_{x 2}^{T}(t), \ldots, \theta_{x n}^{T}(t)\right\}$ is the adaptively updated estimate of the unknown parameter $\Theta_{x}^{* T}$.

Controller structure. We choose the controller $u(t)$ as

$$
u(t)=K_{x}^{T}(t) \psi_{x}(t)+k_{2}(t) r(t)
$$

where $K_{x}^{T}(t)=K_{1}^{T}(t) \Theta_{x}^{T}(t)$ and $k_{2}(t)$ are the estimates of the unknown nominal parameters $K_{x}^{* T}=K_{1}^{* T} \Theta_{x}^{* T}$ and $k_{2}^{*}$. The nominal parameters $K_{1}^{*}$ and $k_{2}^{*}$ satisfy the following plant-model matching equations:

$$
\operatorname{det}\left(s I-A-B K_{1}^{* T}\right)=\frac{Z(s)}{z_{m} W_{m}(s)}, k_{2}^{*-1}=z_{m} .
$$

Closed-loop system. Substituting the controller signal (12.2.9) in the plant (12.1.1) and applying the state signal model (12.2.7), we have the closed-loop system

$$
\dot{x}=\left(A+B K_{1}^{* T}\right) x+B k_{2}^{*} r+B \widetilde{\Theta}^{T} \omega, \quad y=C x
$$

where $\widetilde{\Theta}(t)=\Theta(t)-\Theta^{*}, \Theta(t)=\left[K_{x}^{T}, k_{2}\right]^{T}, \Theta^{*}=\left[K_{x}^{* T}, k_{2}^{*}\right]^{T}$, and $\omega(t)=\left[\psi_{x}^{T}, r^{T}\right]^{T}$. From the matching equation (12.2.10), if $\Theta(t)=\Theta^{*}$, we have $\lim _{t \rightarrow \infty}\left(y(t)-y_{m}(t)\right)=0$. 
That is, the controller (12.2.9) with nominal parameter $\Theta^{*}$ can make the output track the reference output. However, $\Theta^{*}$ is unknown, we need to apply the controller (12.2.9) with adaptively updated parameter $\Theta(t)$.

To develop an adaptive law for the parameter $\Theta(t)$, the output tracking error information is needed. Since the output sensors have uncertainties, we cannot obtain the exact output signal $y(t)$. An output sensor compensator $\hat{y}(t)$ is introduced to estimate the output signal $y(t)$.

Output sensor compensator. From the output sensor uncertainty model (12.2.4), we retrieve that

$$
y(t)=\theta_{y}^{* T} \psi_{y}(t)
$$

where $\theta_{y}^{*}=\left[\theta_{k y}^{*}, \theta_{b y 1}^{*}, \ldots, \theta_{b y p}^{*}\right]^{T}$ and $\psi_{y}(t)=\left[z_{y}(t), f_{y 1}(t), \ldots, f_{y p}(t)\right]^{T}$ with $\theta_{k y}^{*}=1 / k_{y}$ and $\theta_{b y j}^{*}=-b_{y j} / k_{y}, j=1,2, \ldots, p_{i}$ being unknown constant parameters. Then, the output compensation signal $\hat{y}(t)$ is given as

$$
\hat{y}(t)=\theta_{y}^{T}(t) \psi_{y}(t)
$$

where $\theta_{y}(t)$ is an estimate of the unknown parameter $\theta_{y}^{*}$.

Compensation tracking error. We introduce a compensation output tracking error signal as

$$
\hat{e}(t)=\hat{y}(t)-y_{m}(t)=e(t)+(\hat{y}(t)-y(t))
$$

where $e(t)=y(t)-y_{m}(t)$ is the actual output tracking error.

In view of the closed-loop system (12.2.11), the reference system (12.1.3), and the matching condition (12.2.10), we have

$$
\hat{e}(t)=\rho^{*} W_{m}(s)\left[\widetilde{\Theta}^{T} \omega\right](t)+\tilde{\theta}_{y}^{T}(t) \psi_{y}(t),
$$

where $\rho^{*}=z_{m}$ and $\tilde{\theta}_{y}(t)=\theta_{y}(t)-\theta_{y}^{*}$. 
Estimation error. We introduce an estimation error as

$$
\hat{\epsilon}(t)=\hat{e}(t)+\rho(t) \xi(t)
$$

where $\rho(t)$ is an estimate of the unknown $\rho^{*}$ and

$$
\xi(t)=\Theta^{T}(t) \zeta(t)-W_{m}(s)\left[\Theta^{T} \omega\right](t)
$$

with $\zeta(t)=W_{m}(s)[\omega](t)$. Substituting (12.2.15) in (12.2.16), we have

$$
\hat{\epsilon}(t)=\rho^{*} \widetilde{\Theta}^{T}(t) \zeta(t)+\tilde{\rho}(t) \xi(t)+\tilde{\theta}_{y}^{T}(t) \psi_{y}(t)
$$

where $\tilde{\rho}(t)=\rho(t)-\rho^{*}$.

Adaptive laws. With the estimation error model (12.2.18), adaptive laws for $\Theta(t), \rho(t)$, and $\theta_{y}(t)$ are chosen as

$$
\begin{aligned}
\dot{\Theta}(t) & =-\frac{\Gamma \operatorname{sign}\left(z_{m}\right) \zeta(t) \hat{\epsilon}(t)}{m^{2}(t)}, \\
\dot{\rho}(t) & =-\frac{\gamma \xi(t) \hat{\epsilon}(t)}{m^{2}(t)} \\
\dot{\theta}_{y}(t) & =-\frac{\Gamma_{y} \psi_{y}(t) \hat{\epsilon}(t)}{m^{2}(t)}
\end{aligned}
$$

where $\hat{\epsilon}(t)$ is computed from (12.2.16), in which $\hat{e}(t)$ is computed from $\hat{e}(t)=\Theta_{y}^{T}(t) \psi_{y}(t)-$ $y_{m}(t), \Gamma=\Gamma^{T}>0$ and $\Gamma_{y}=\Gamma_{y}^{T}>0$ are adaptation gain matrices, and

$$
m(t)=\left(1+\xi^{2}(t)+\zeta^{T}(t) \zeta(t)+\psi_{y}^{T}(t) \psi_{y}(t)\right)^{1 / 2}
$$

is a standard normalization signal.

The sensor uncertainty compensation scheme with the control law (12.2.9) updated by the adaptive laws (12.2.19)-(12.2.21), when applied to the plant (12.1.1), guarantees the closed-loop signal boundedness and asymptotic compensation output tracking: $\lim _{t \rightarrow \infty}\left(\hat{y}(t)-y_{m}(t)\right)=0$. 
The convergence of the actual tracking error $e(t)=y(t)-y_{m}(t)$ to zero is under investigation and it may need some additional conditions in the adaptive control system, as similar to an adaptive observer case where the adaptive state estimation error converges to zero under some persistent excitation conditions in the case when $(A, B)$ are unknown.

\subsubsection{Sensor Uncertainty Detection Designs}

Since the adaptive state feedback controller (12.2.9) with the adaptive laws (12.2.19)(12.2.21) can ensure the boundedness of the closed-loop signals, the bounded control input signal $u(t)$, state sensor signal $z_{x}(t)$, and output sensor signal $z_{y}(t)$ are used to construct detector models. By observing residuals between the sensor signals $\left(z_{x}(t)\right.$ or $\left.z_{y}(t)\right)$ and the corresponding detector model signals, we can determine whether there exist sensor uncertainties.

\subsubsection{State sensor uncertainty detection scheme}

Based on the state sensor dynamic model (12.2.2), we start with design and analysis of a benchmark detection model system which will be used to develop a bank of detector model systems for different sensor uncertainty patterns.

Total uncertainty sensor estimation model. We introduce an estimation model to estimate the unknown parameters in sensor dynamic model (12.2.2), which is given as

$$
\dot{z}_{m}=A_{m} z_{m}+\left(\widehat{A}_{z}-A_{m}\right) z_{x}+\widehat{B}_{z} u+\widehat{\Theta}_{z}^{T} f_{x}+\widehat{\Theta}_{b x}^{T} \dot{f}_{x}
$$

where $A_{m}$ is a chosen stable matrix, and $\widehat{A}_{z}(t), \widehat{B}_{z}(t), \widehat{\Theta}_{z}(t)$, and $\widehat{\Theta}_{b x}(t)$ are the adaptive estimates of $A_{z}, B_{z}, \Theta_{z}$, and $\Theta_{b x}$ in the total uncertainty dynamic model (12.2.2). From (12.2.2) and (12.2.22), we obtain the error dynamic system as

$$
\dot{e}_{x}=A_{m} e_{x}+\widetilde{A}_{z} z_{x}+\widetilde{B}_{z} u+\widetilde{\Theta}_{z}^{T} f_{x}+\widetilde{\Theta}_{b x}^{T} \dot{f}_{x}
$$


where $e_{x}(t)=z_{m}(t)-z_{x}(t), \widetilde{A}_{z}(t)=\widehat{A}_{z}(t)-A_{z}, \widetilde{B}_{z}(t)=\widehat{B}_{z}(t)-B_{z}, \widetilde{\Theta}_{z}(t)=$ $\widehat{\Theta}_{z}(t)-\Theta_{z}, \widetilde{\Theta}_{b x}(t)=\widehat{\Theta}_{b x}(t)-\Theta_{b x}$. Then, the adaptive laws are chosen as

$$
\begin{aligned}
& \dot{\hat{A}_{z}}=-\Gamma_{1} P e_{x} z_{x}^{T}, \quad \dot{\widehat{B}}_{z}=-\Gamma_{2} P e_{x} u, \\
& \dot{\Theta}_{z}^{T}=-\Gamma_{3} P e_{x} f_{x}^{T}, \quad \widehat{\Theta}_{b x}^{T}=-\Gamma_{4} P e_{x} \dot{f}_{x}^{T},
\end{aligned}
$$

where $\Gamma_{i}=\Gamma_{i}^{T}>0, i=1,2,3,4, P=P^{T}>0$ satisfying $P A_{m}+A_{m}^{T} P=-Q$ with $Q=Q^{T}>0$.

Since the proposed adaptive control law (12.2.9) ensures that $z_{x}(t)$ and $u(t)$ are bounded, we have

Proposition 12.2.1. Given that the signals $z_{x}(t), u(t), f_{x}(t)$, and $\dot{f}_{x}(t)$ are bounded, the proposed estimation model system (12.2.22) with the adaptive laws (12.2.24) and (12.2.25) ensures that $z_{m}(t), \widehat{A}_{z}(t), \widehat{B}_{z}(t), \widehat{\Theta}_{z}(t)$, and $\widehat{\Theta}_{b x}(t)$ are bounded, and $\lim _{t \rightarrow \infty} e_{x}(t)=\lim _{t \rightarrow \infty}\left(z_{m}(t)-z_{x}(t)\right)=0$, when $z_{x}(t)$ is of the total sensor uncertainty pattern (12.2.1).

The proof of this result is standard. Consider a positive definite function

$$
\begin{aligned}
V= & e_{x}^{T} P e_{x}+\operatorname{tr}\left[\widetilde{A}_{z}^{T} \Gamma_{1}^{-1} \widetilde{A}_{z}\right]+\left[\widetilde{B}_{z}^{T} \Gamma_{2}^{-1} \widetilde{B}_{z}\right] \\
& +\operatorname{tr}\left[\widetilde{\Theta}_{z} \Gamma_{3}^{-1} \widetilde{\Theta}_{z}^{T}\right]+\operatorname{tr}\left[\widetilde{\Theta}_{b x} \Gamma_{4}^{-1} \widetilde{\Theta}_{b x}^{T}\right]
\end{aligned}
$$

From the adaptive laws (12.2.24) and (12.2.25), we obtain its time-derivative as

$$
\dot{V}=-e_{x}^{T}(t) Q e_{x}(t) \leq 0
$$

Then, the properties in Proposition 12.2.1 can be derived.

The above estimation model is designed for the general uncertainty signal $\Theta_{b x}^{T} f_{x}(t)$ in the state sensor model (12.2.1). For a specific situation, some of the terms $\theta_{b x i}^{T} f_{x i}(t)$ may be not in the sensor uncertainty model (12.2.1), that is the corresponding parameter $\theta_{b x i}=0$. To identify the specific sensor uncertainty patterns, some partial 
sensor uncertainty estimation model system will be designed. A special one is for the case when no sensor bias uncertainty is present, i.e. $\Theta_{b x}^{T} f_{x}(t)=0$ in the sensor uncertainty model (12.2.1).

Bias-uncertainty free sensor estimation model. The sensor dynamic model without bias-uncertainties is given as

$$
\dot{z}_{x}(t)=A_{z} z_{x}(t)+B_{z} u(t)
$$

Based on (12.2.28), we design a bias-uncertainty free sensor estimation model:

$$
\dot{z}_{m}(t)=A_{m} z_{m}(t)+\left(\widehat{A}_{z}(t)-A_{m}\right) z_{x}(t)+\widehat{B}_{z}(t) u(t)
$$

where $\widehat{A}_{z}(t)$ and $\widehat{B}_{z}(t)$ are updated from the adaptive laws in (12.2.24). This estimation model has similar properties to that in Proposition 12.2.1, in particular, $\lim _{t \rightarrow \infty}\left(z_{m}(t)-z_{x}(t)\right)=0$, for the bias-uncertainty free sensor dynamic model (12.2.28). On the other hand, when the sensor has bias-uncertainties such as the model (12.2.2), the tracking property may not hold, that is $z_{m}(t)-z_{x}(t)$ does not converge to 0 .

Therefore, the bias-uncertainty free sensor output estimation model can be used to detect the sensor bias-uncertainties. The detection criterion is that if $z_{m}(t)$ from the bias-uncertainty free estimation model (12.2.29) cannot track the state sensor signal $z_{x}(t)$, the state sensors have bias-uncertainties.

To identify which state sensor has bias-uncertainty, we need to design a bank of uncertainty-specific sensor output estimation model systems.

Uncertainty-specific sensor estimation models. The bias-uncertainty model in $(12.2 .1)$ :

$$
\Theta_{b x}^{T} f_{x}(t)=\left[\theta_{b x 1}^{T} f_{x 1}(t), \theta_{b x 2}^{T} f_{x 2}(t), \ldots, \theta_{b x n}^{T} f_{x n}(t)\right]^{T}
$$

contains all possible cases of sensor uncertainties with $\theta_{b x i}=0$ or not. To identify and isolate some uncertainty patterns where a part of the state sensors do not have bias- 
uncertainty, i.e. $\theta_{b x i}=0$, for $i=j_{1}, j_{2}, \ldots, j_{k}$ with $\left\{j_{1}, j_{2}, \ldots, j_{k}\right\} \subset\{1,2, \ldots, n\}$, while others have bias-uncertainty, we need to construct some estimation models to ensure that the error $e_{x}(t)=z_{m}(t)-z_{x}(t)$ converges to zero only when $z_{x}(t)$ is of the specific uncertainty patterns.

Then, we will give an illustrative design to isolate the uncertainty pattern where $\theta_{b x 2} \neq 0, \theta_{b x 4} \neq 0$, and $\theta_{b x i}=0$, for $i=1,3,5,6, \ldots, n$, i.e. only the 2 nd and the 4 th sensors have bias-uncertainties. For this particular uncertain sensor signal $z_{x}(t)$, we have

$$
\Theta_{b x}^{T} f_{x}(t)=\left[0, \theta_{b x 2}^{T} f_{x 2}(t), 0, \theta_{b x 4}^{T} f_{x 4}(t), 0, \ldots, 0\right]^{T}
$$

Then the state sensor dynamics (12.2.2) becomes

$$
\begin{aligned}
\dot{z}_{x}(t)= & A_{z} z_{x}(t)+B_{z} u(t)+\Theta_{z 2}^{T} f_{x 2}(t)+\Theta_{z 4}^{T} f_{x 4}(t) \\
& +\left[0, \theta_{b x 2}^{T} \dot{f}_{x 2}(t), 0, \theta_{b x 4}^{T} \dot{f}_{x 4}(t), 0, \ldots, 0\right]^{T}
\end{aligned}
$$

where $\Theta_{z 2}^{T}=a_{2} \theta_{b x 2}^{T}$ with $a_{2}$ being the second column of $-K_{x} A K_{x}^{-1}$ and $\Theta_{z 4}^{T}=a_{4} \theta_{b x 4}^{T}$ with $a_{4}$ being the fourth column of $-K_{x} A K_{x}^{-1}$. Based on (12.2.32), we choose the estimation model as

$$
\begin{gathered}
\dot{z}_{m}=A_{m} z_{m}+\left(\widehat{A}_{z}-A_{m}\right) z_{x}+\widehat{B}_{z} u+\widehat{\Theta}_{z 2}^{T} f_{x 2}+\widehat{\Theta}_{z 4}^{T} f_{x 4} \\
+\left[0, \hat{\theta}_{b x 2}^{T} \dot{f}_{x 2}, 0, \hat{\theta}_{b x 4}^{T} \dot{f}_{x 4}, 0, \ldots, 0\right]^{T} .
\end{gathered}
$$

We obtain the error dynamics from (12.2.32) and (12.2.33) as

$$
\begin{aligned}
\dot{e}_{x}= & A_{m} e_{x}+\widetilde{A}_{z} z_{x}+\widetilde{B}_{z} u+\widetilde{\Theta}_{z 2}^{T} f_{x 2}+\widetilde{\Theta}_{z 4}^{T} f_{x 4} \\
& +\left[0, \tilde{\theta}_{b x 2}^{T} \dot{f}_{x 2}, 0, \tilde{\theta}_{b x 4}^{T} \dot{f}_{x 4}, 0, \ldots, 0\right]^{T}
\end{aligned}
$$

where $\widetilde{\Theta}_{z i}=\widehat{\Theta}_{z i}-\Theta_{z i}$ and $\tilde{\theta}_{b x i}=\hat{\theta}_{b x i}-\theta_{b x i}$, for $i=2,4$. 
The adaptive laws for $\widehat{A}_{z}$ and $\widehat{B}_{z}$ are chosen as (12.2.24), and the adaptive laws for $\widehat{\Theta}_{z i}$ and $\hat{\theta}_{b x i}(i=2,4)$ are chosen as

$$
\begin{aligned}
& \dot{\hat{\Theta}}_{z 2}^{T}=-\Gamma_{5} P e_{x} f_{x 2}^{T}, \quad \dot{\hat{\Theta}}_{z 4}^{T}=-\Gamma_{6} P e_{x} f_{x 4}^{T}, \\
& \dot{\hat{\theta}}_{b x 2}^{T}=-\Gamma_{7} p_{2}^{T} e_{x} \dot{f}_{x 2}^{T}, \quad \dot{\hat{\theta}}_{b x 4}^{T}=-\Gamma_{8} p_{4}^{T} e_{x} \dot{f}_{x 4}^{T},
\end{aligned}
$$

where $\Gamma_{i}=\Gamma_{i}^{T}>0, i=5,6,7,8, P=P^{T}>0$ satisfying $P A_{m}+A_{m}^{T} P=-Q$ with $Q=Q^{T}>0$, and $p_{i}, i=2,4$, is the $i$ th column of $P$.

Similar with Proposition 12.2.1, the residual converges to 0: $\lim _{t \rightarrow \infty}\left(z_{m}-z_{x}\right)=0$ when the bias-uncertainty pattern is $\Theta_{b x}^{T} f_{x}(t)=\left[0, \theta_{b x 2}^{T} f_{x 2}(t), 0, \theta_{b x 4}^{T} f_{x 4}(t), 0, \ldots, 0\right]^{T}$. Then, we have the following detection criteria.

If the residual $e_{x}=z_{m}-z_{x}$ converges to zero, where $z_{m}$ is from the estimation model (12.2.33), we can obtain that there exist three possible patterns for the sensor $z_{x}=\left[z_{x 1}, z_{x 2}, \ldots, z_{x n}\right]^{T}:$

(i) all the state sensors $z_{x i}$ do not have the bias-uncertainty terms;

(ii) either $z_{x 2}$ has the bias-uncertainty term $\theta_{b x 2}^{T} f_{x 2}$ or $z_{x 4}$ has the bias-uncertainty term $\theta_{b x 4}^{T} f_{x 4}$

(iii) both $z_{x 2}$ and $z_{x 4}$ have bias-uncertainty terms.

To further isolate the sensor uncertainty pattern, we need to observe the residuals $e_{x}$ obtained from the bias-uncertainty free estimation model (12.2.29) and the estimation models corresponding to the sensor uncertainty patterns: $\Theta_{b x}^{T} f_{x}=\left[0, \theta_{b x 2}^{T} f_{x 2}, 0, \ldots, 0\right]^{T}$ and $\Theta_{b x}^{T} f_{x}(t)=\left[0,0,0, \theta_{b x 4}^{T} f_{x 4}, 0, \ldots, 0\right]^{T}$ respectively. The isolation of particular uncertainty patterns will be shown in the simulation. 


\subsubsection{Output sensor uncertainty detection scheme}

Since the system only has one output signal, we only detect whether the output sensor has bias-uncertainty. Based on the output sensor model (12.2.6), we have the following design.

Total uncertainty estimation model. We introduce an estimation model system to estimate the parameters in (12.2.6):

$$
\hat{z}_{y}=\hat{\theta}_{u}^{T} \phi_{u}+\hat{\theta}_{z}^{T} \phi_{z}+\hat{\theta}_{b f}^{T} f_{y}+\sum_{i=1}^{p} \hat{\theta}_{i}^{T} \phi_{i}
$$

where $\hat{\theta}_{u}(t), \hat{\theta}_{z}(t), \hat{\theta}_{b f}(t)$, and $\hat{\theta}_{i}(t), i=1, \ldots, p$ are the estimates of corresponding unknown parameters. Then, the estimation error $e_{y}(t)=\hat{z}_{y}(t)-z_{y}(t)$ is obtained as

$$
e_{y}=\tilde{\theta}_{u}^{T} \phi_{u}+\tilde{\theta}_{z}^{T} \phi_{z}+\tilde{\theta}_{b f}^{T} f_{y}+\sum_{i=1}^{p} \tilde{\theta}_{i}^{T} \phi_{i}
$$

where $\tilde{\theta}_{u}(t), \tilde{\theta}_{z}(t), \tilde{\theta}_{b f}(t)$, and $\tilde{\theta}_{i}(t)$ for $i=1,2, \ldots, p$, are the parameter errors. We choose the adaptive laws as

$$
\begin{aligned}
& \dot{\hat{\theta}}_{u}=-\frac{\Gamma_{u} \phi_{u} e_{y}}{m^{2}}, \quad \dot{\hat{\theta}}_{z}=-\frac{\Gamma_{z} \phi_{z} e_{y}}{m^{2}} \\
& \dot{\hat{\theta}}_{b f}=-\frac{\Gamma_{b f} \phi_{b f} e_{y}}{m^{2}(t)}, \quad \dot{\hat{\theta}}_{i}=-\frac{\Gamma_{f i} \phi_{i} e_{y}}{m^{2}}
\end{aligned}
$$

where $\Gamma_{u}, \Gamma_{z}, \Gamma_{b f}$, and $\Gamma_{f i}, i=1,2, \ldots, p$, are positive definite and symmetric gain matrices, and

$$
m(t)=\left(1+\phi_{u}^{T} \phi_{u}+\phi_{z}^{T} \phi_{z}+\phi_{b f}^{T} \phi_{b f}+\sum_{i=1}^{p} \phi_{i}^{T} \phi_{i}\right)^{1 / 2}
$$

Proposition 12.2.2. Given that the signals $z_{y}(t), u(t), f_{y}(t)$, and $\dot{f}_{y}(t)$ are bounded, the proposed estimation model system (12.2.37) with the adaptive laws (12.2.39)(12.2.40) ensures that $\lim _{t \rightarrow \infty} e_{y}(t)=\lim _{t \rightarrow \infty}\left(\hat{z}_{y}(t)-z_{y}(t)\right)=0$, when $z_{y}(t)$ is of the total sensor uncertainty pattern (12.2.6). 
Bias-uncertainty free sensor estimation model. The above estimation model (12.2.37) is based on the total sensor uncertainty pattern (12.2.6). To detect the no bias-uncertainty case, i.e. $z_{y}(t)=k_{y} y(t)$, we build the estimation model as

$$
\hat{z}_{y}(t)=\hat{\theta}_{u}^{T}(t) \phi_{u}(t)+\hat{\theta}_{z}^{T}(t) \phi_{z}(t)
$$

where $\hat{\theta}_{u}(t)$ and $\hat{\theta}_{z}(t)$ are updated from the adaptive laws in (12.2.39). This estimation model has similar properties to that in Proposition 2, which is $\lim _{t \rightarrow \infty}\left(\hat{z}_{y}(t)-z_{y}(t)\right)=$ 0 , for the case when $\theta_{b y}^{T} f_{y}(t)=0$ in the sensor model (12.2.4). When there is biasuncertainty, the tracking property may not hold, that is $\lim _{t \rightarrow \infty}\left(\hat{z}_{y}(t)-z_{y}(t)\right) \neq 0$. Thus, the detection criterion is that if $\hat{z}_{y}(t)$ from the bias-uncertainty free estimation model (12.2.41) cannot track the output sensor signal $z_{y}(t)$, the output sensor has bias-uncertainty.

\subsection{Simulation Study}

In this simulation study, we consider a linearized aircraft longitudinal dynamic model with sensor uncertainties and system parameter uncertainties.

Longitudinal aircraft model. The linearized aircraft longitudinal model can be described as

$$
\dot{x}(t)=A x(t)+B u(t), \quad y(t)=C x(t)
$$

with state and input variables: $x=\left[u_{b}, w_{b}, q_{b}, \theta\right]^{T}$ and $u=d_{e}$, where $u_{b}$ and $w_{b}$ are the body-axis velocity components of the origin of the body-axis frame whose units are $\mathrm{ft} / \mathrm{sec}, q_{b}$ is the body-axis component of the angular velocity whose unit is $\mathrm{deg} / \mathrm{sec}, \theta$ is the Euler pitch angle whose unit is degree, and $d_{e}$ is the elevator angular position whose unit is degree.

In this study, we choose the pitch angle $\theta(t)$ as the output signal $y(t)$, so the 
matrix $C$ is given as

$$
C=\left[\begin{array}{llll}
0 & 0 & 0 & 1
\end{array}\right]
$$

Although the parameters in $A$ and $B$ have uncertainties, the structures can be obtained from the generic aircraft dynamics, which are given as

$$
A=\left[\begin{array}{rrrr}
a_{11} & a_{12} & a_{13} & a_{14} \\
a_{21} & a_{22} & a_{23} & a_{24} \\
a_{31} & a_{32} & a_{33} & 0 \\
0 & 0 & 1 & 0
\end{array}\right], B=\left[\begin{array}{r}
b_{11} \\
b_{21} \\
b_{31} \\
0
\end{array}\right]
$$

Sensor uncertainty models. We consider a state sensor uncertainty model

$$
\begin{aligned}
& z_{x 1}(t)=x_{1}(t), \quad z_{x 2}(t)=k_{x 2} x_{2}(t)+b_{x 1} \sin (t) \\
& z_{x 3}(t)=x_{3}(t), \quad z_{x 4}(t)=k_{x 4} x_{4}(t)+b_{x 4} \sin (2 t)
\end{aligned}
$$

where only the 2 nd and 4 th state sensors have bias-uncertainties. Since the state signal $x_{4}=\theta$ is the output signal, the sensor $z_{x 4}$ is also used as an output sensor.

Verification of control design conditions. From the structure information (12.3.2) and (12.3.3), we can obtain that the system is controllable and observable, the degree of $Z(s)$ in (12.1.4) is $m=2$ and $z_{m}=b_{31}$. Based on the physical meaning of the parameter $b_{31}$, which is a control gain from elevator to pitch acceleration, we have $b_{31}<0$, that is $\operatorname{sign}\left(z_{m}\right)=\operatorname{sign}\left(b_{31}\right)=-1$. The minimum phase condition (A1) can be verified by the numerical values of $A$ and $B$, which are used to build the simulation system.

Since the design conditions hold for the aircraft flight system, we apply the proposed adaptive controller (12.2.9) to the longitudinal aircraft model (12.3.1) with sensor uncertainties (12.3.4) to ensure the closed-loop signal boundedness. Then, we can use the sensor estimation models to detect and identify the sensor uncertainties.

Simulation results of uncertainty detection. Since the state sensor for pitch 
angle $\theta$ is also used as the output sensor, in this simulation study, we only consider the state sensor uncertainty detection problem.

To detect the sensor uncertainties of (12.3.4) and also identify that only the 2nd and 4th sensors have bias-uncertainties, we observe four sensor estimation models:

(i) bias-uncertainty free sensor estimation model (12.2.29);

(ii) uncertainty-specific sensor estimation model (12.2.33) for the case when $\Theta_{b x}^{T} f_{x}(t)=$ $\left[0, \theta_{b x 2}^{T} f_{x 2}(t), 0, \theta_{b x 4}^{T} f_{x 4}(t)\right]^{T}$

(iii) uncertainty-specific sensor estimation model (12.2.33) for the case when $\Theta_{b x}^{T} f_{x}(t)=$ $\left[0, \theta_{b x 2}^{T} f_{x 2}(t), 0,0\right]^{T}$

(iv) uncertainty-specific sensor estimation model (12.2.33) for the case when $\Theta_{b x}^{T} f_{x}(t)=$ $\left[0,0,0, \theta_{b x 4}^{T} f_{x 4}(t)\right]^{T}$.

Fig. 12.1 shows the residual $e_{x}(t)=z_{m}(t)-z_{x}(t)$ obtained from the estimation model (i), which does not converge to zero. From Fig. 12.1, we can see that there exist uncertainties in the state sensors. Fig. 12.2 shows the residual $e_{x}(t)$ obtained from the estimation model (ii), which converges to zero, which means that there are uncertainties in the 2 nd state sensor or the 4 th state sensor. Since the residual $e_{x}(t)$ in Fig. 12.3 obtained from the estimation model (iii) and the residual $e_{x}(t)$ in Fig. 12.4 obtained from the estimation model (iv) do not converge to zero, we can conclude that both the 2 nd and 4 th sensors have bias-uncertainties.

\section{Summary}

This chapter addressed the design, analysis and evaluation of the adaptive feedbackbased sensor uncertainty detection scheme. The sensor dynamics has been derived based on the parametric sensor uncertainty model, and a set of sensor estimation 

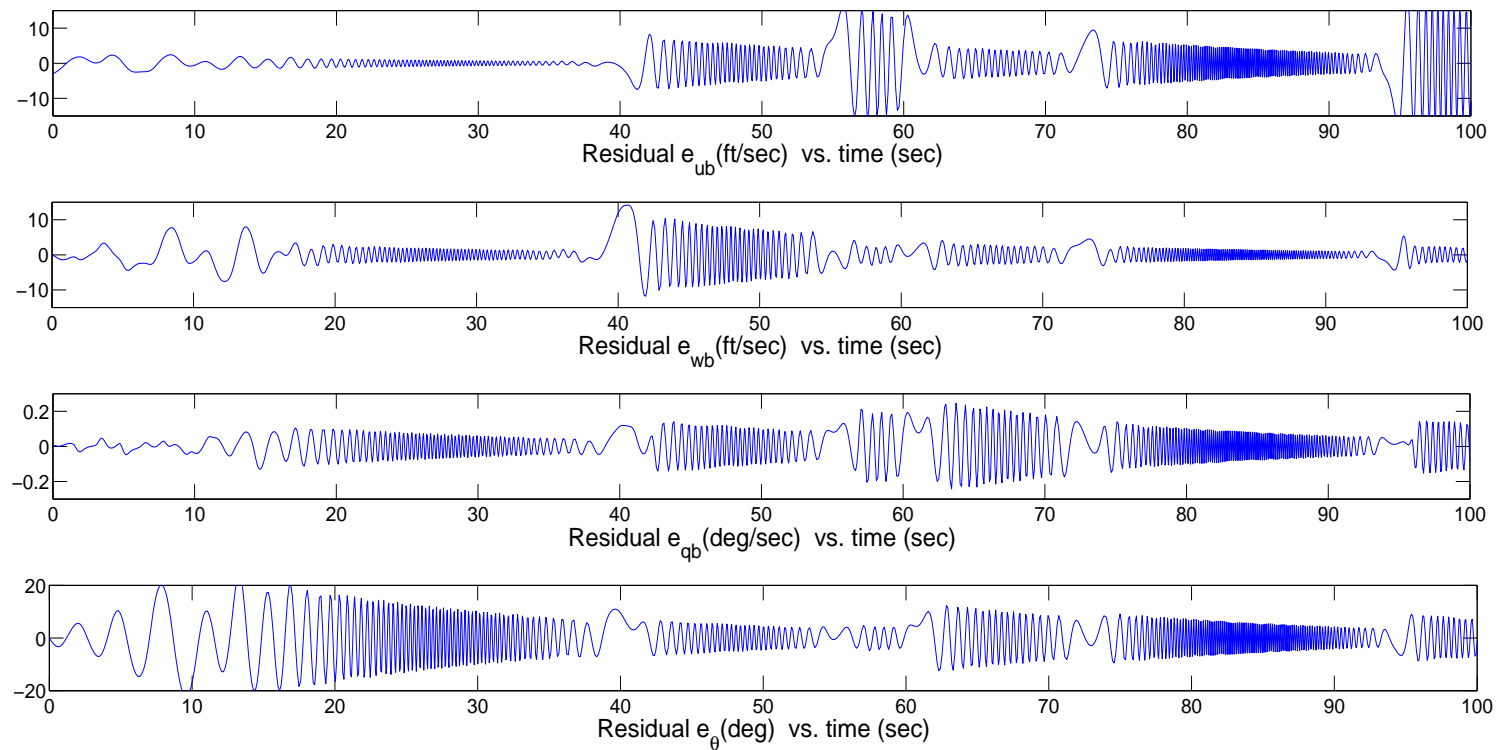

Figure 12.1: Residual $e_{x}(t)=z_{m}(t)-z_{x}(t)$ for the estimation model (i).
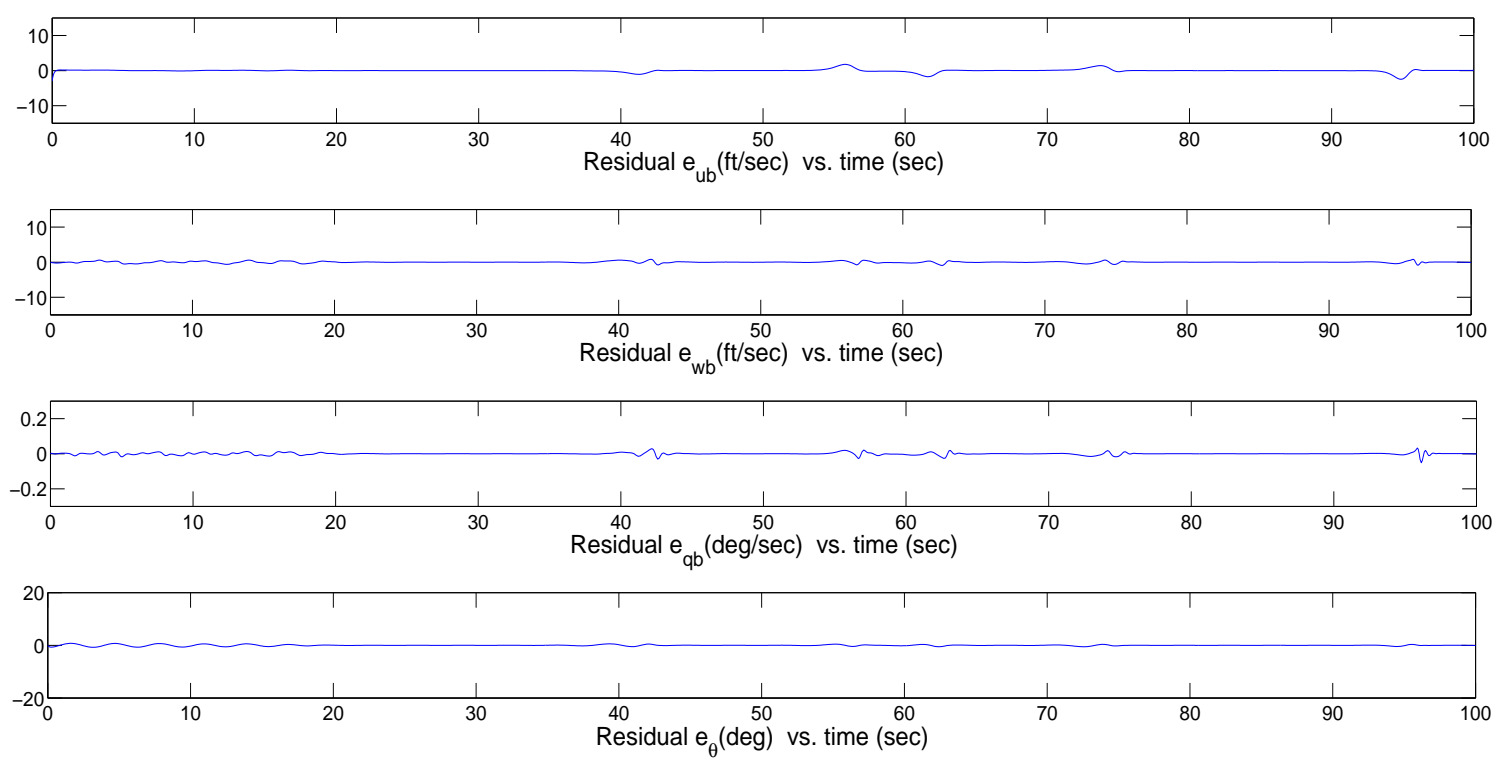

Figure 12.2: Residual $e_{x}(t)=z_{m}(t)-z_{x}(t)$ for the estimation model (ii). 

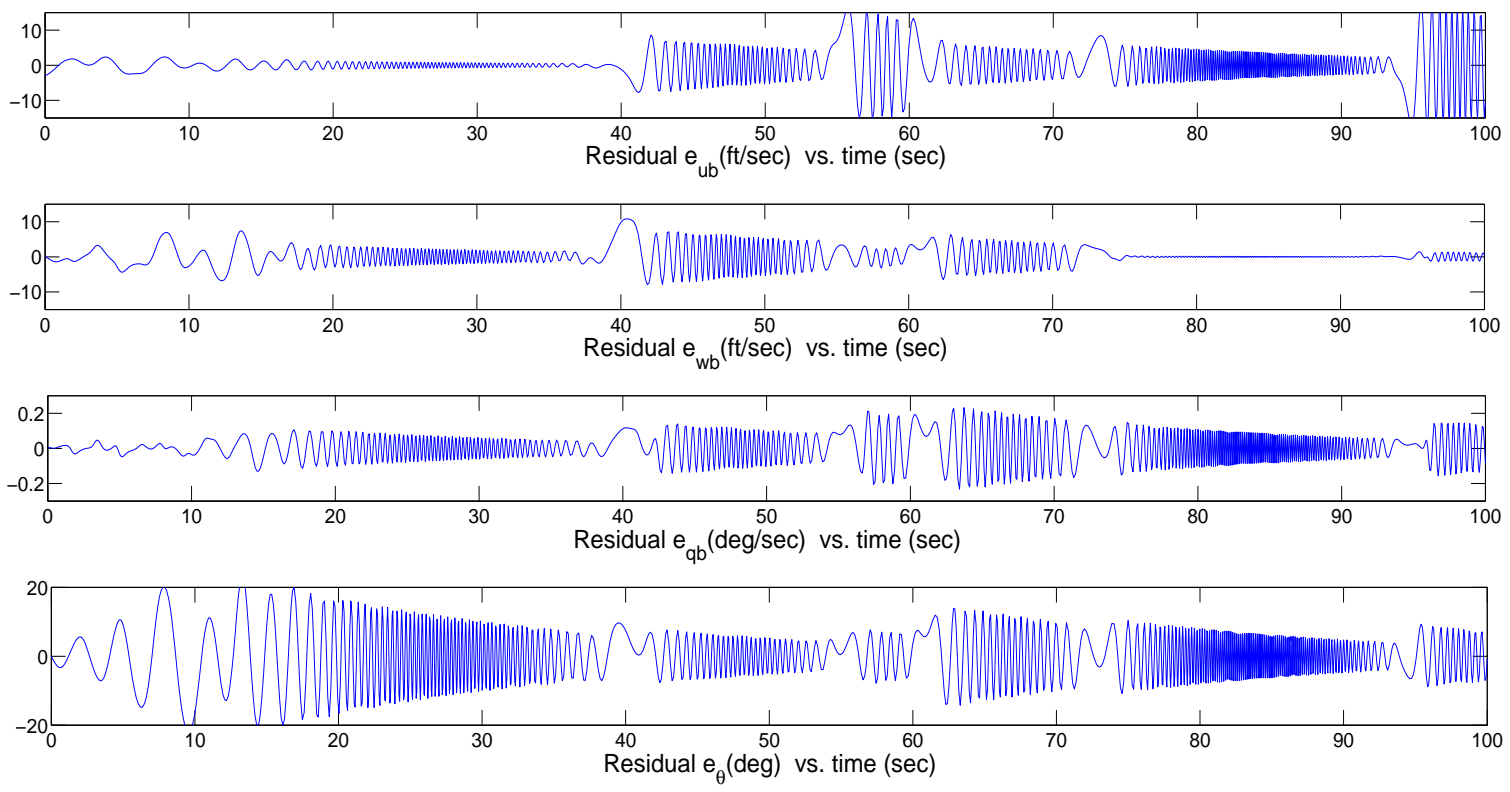

Figure 12.3: Residual $e_{x}(t)=z_{m}(t)-z_{x}(t)$ for the estimation model (iii).
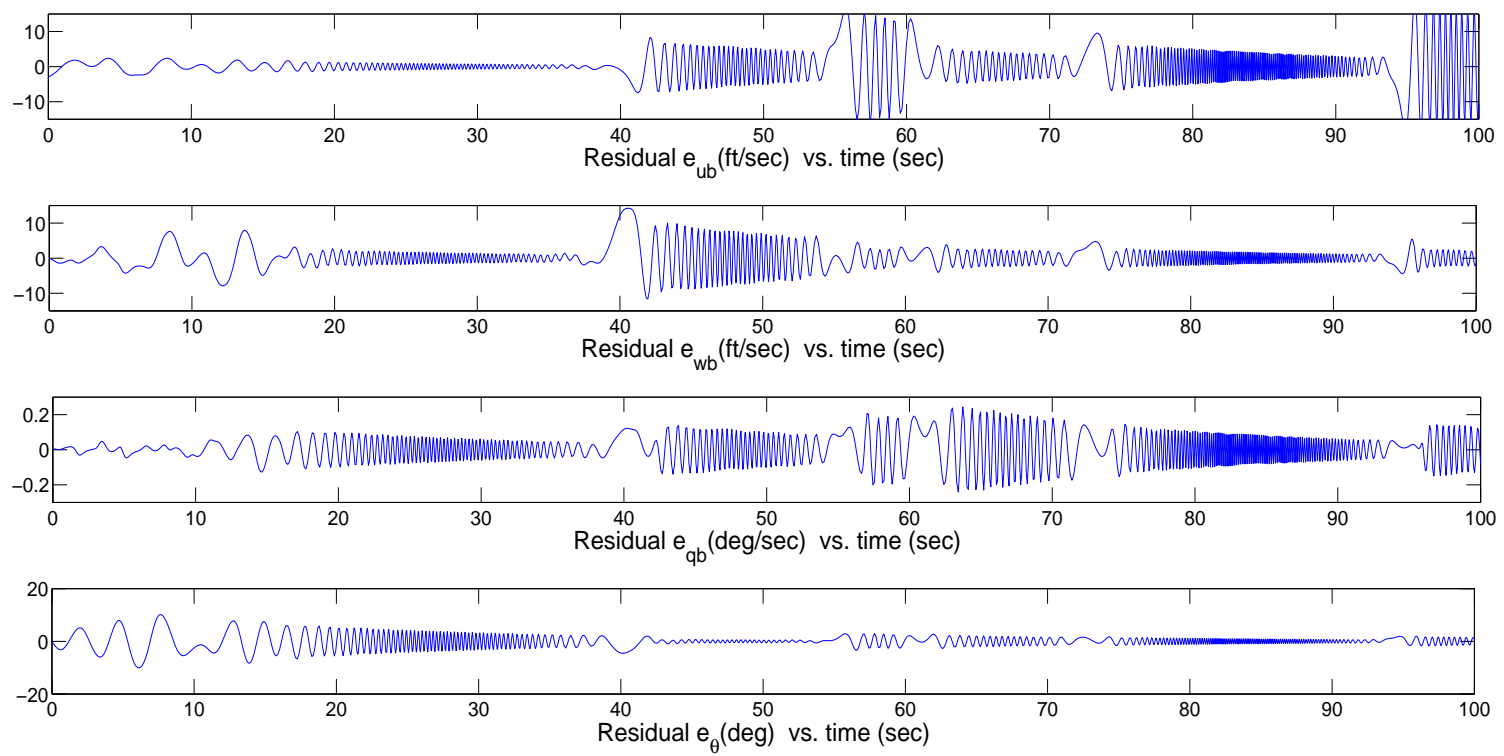

Figure 12.4: Residual $e_{x}(t)=z_{m}(t)-z_{x}(t)$ for the estimation model (iv). 
model systems have been constructed to estimate the uncertain sensor signals. To ensure the signal boundedness requirement of the estimation model, the adaptive feedback control design has been applied to the system with the sensor uncertainty. By comparing the estimation model signals with the sensor signals, we can determine whether there are sensor uncertainties or not, and the uncertainty patterns can also be identified. The simulation study of the linearized longitudinal aircraft system showed the effectiveness of the proposed feedback-based sensor uncertainty design. 


\section{Chapter 13}

\section{Conclusions}

This research mainly focus on development of adaptive fault-tolerant control and fault detection designs for multi-input and multi-output systems under damage and component failure conditions with applications to the nonlinear aircraft flight system. For the fault-tolerant adaptive control, it has been shown that the essential condition for multivariable model reference adaptive control designs, namely, the infinite zero structure, can remain invariant under failure and damage conditions. Under such an invariant property, state feedback and output feedback for output tracking designs can be developed to compensate damage and failures. Equipped with the feedback adaptive fault-tolerant control, bounded control input signals and system state signals are used to construct adaptive detector models based on system models under different damage and failure patterns to identify and isolate the fault conditions. Thorough evaluation studies of the nonlinear NASA generic transport model have been conducted to show the effectiveness of the developed linearization-based adaptive fault-tolerant control and fault detection schemes for the nonlinear system around the neighborhood of the chosen operating points. The detailed research topics included in this dissertation are concluded as follows:

- Multivariable state feedback for output tracking MRAC of MIMO systems under 
parametric uncertainties with application to the GTM (Chapter 3).

- Adaptive structural damage compensation design using multivariable state feedback for output tracking MRAC with application to the GTM (Chapter 4).

- Adaptive simultaneous actuator failure and structural damage compensation design using multivariable output feedback for output tracking MRAC with application to the GTM (Chapter 5).

- Adaptive sensor failure compensation design using multivariable state feedback for output tracking MRAC with application to the GTM (Chapter 6).

- Discrete-time multivariable adaptive control design for damage compensation of nonlinear systems with application to the GTM (Chapter 7).

- Adaptive output feedback actuator nonlinearity compensation design with applications to aircraft systems using synthetic jet actuators (Chapter 8).

- Adaptive state feedback disturbance rejection for MIMO linear time-invariant systems and adaptive output feedback disturbance rejection for MIMO piecewise linear systems (Chapter 9 and Chapter 10).

- Feedback-based adaptive structural damage detection and sensor uncertainty detection designs with applications to the GTM (Chapter 11 and Chapter 12).

For our future research, we may focus on expanding the operation regions of such linearization-based adaptive fault-tolerant control designs for the nonlinear systems. A possible method is to approximate the nonlinear system as a global linear timevarying model constructed by linearizing the nonlinear system at different operating points with some continuous indicator functions for more precise approximation (which may be different from the piecewise linear system models in Chapter 10), then develop adaptive fault-tolerant control designs based on such a global linear model. 


\section{Bibliography}

[1] Ahmed-Zaid, F., P. Ioannou, K. Gousman, and R. Rooney, "Accommodation of failures in the F-16 aircraft using adaptive control," IEEE Control Systems Magazine, vol. 11, no. 1, pp. 73-78, 1991.

[2] Åström, K. J. and B. Wittenmark, Adaptive Control, 2nd ed., Addison-Wesley, Reading, MA, 1995.

[3] Antsaklis, P. J. and A. N. Michel, Linear Systems, The McGraw-Hill Companies, 1997.

[4] Amato, F., C. Cosentino, M. Mattei and G. Paviglianiti, "A direct/functional redundancy scheme for fault detection and isolation on an aircraft," Aerospace Seience and Technology, 2006, pp. 338-345.

[5] Barbot, J. P., S. Monaco, D. Normand-Cyrot, and N. Pantalos, "Some comments about linearization under sampling," Proceedings of the 31th Conference on Decision and Control, Tucson, Arizona, 1992, pp. 2392-2397.

[6] Bodson, M. and J. E. Groszkiewicz, "Multivariable adaptive algorithms for reconfigurable flight control," IEEE Trans. on Control Systems Technology, vol. 5, no. 2, pp. 217-229, 1997.

[7] Boskovic, J. D., S.-H. Yu, and R. K. Mehra, "A stable scheme for automatic control reconfiguration in the presence of actuator failures," Proceedings of the 1998 ACC, pp. 2455-2459.

[8] Boskovic, J. D., S.-H. Yu, and R. K. Mehra, "Stable adaptive fault-tolerant control of overactuated aircraft using multiple models, switching and tuning," 
Proceedings of the 1998 AIAA Guidance, Navigation and Control Conference, vol. 1, pp. 739-749, 1998.

[9] Boskovic, J. D. and R. K. Mehra, "Stable multiple model adaptive flight control for accommodation of a large class of control effector failures," Proceedings of the 1999 ACC, pp. 1920-1924.

[10] Boskovic, J. D., S. Li, and R. K. Mehra, "Intelligent control of spacecraft in the presence of actuator failures," Proceedings of the 38th IEEE Conference on Decision and Control, pp. 4472-4477, Phoenix, AZ, December 1999.

[11] Bacon, B. J., and Gregory, I. M., "General equations of motion for a damaged asymmetric aircraft," Proceedings of 2007 AIAA Guidance, Navigation, and Control Conference, No. AIAA-2007-6306, Hilton Head, SC, August 2007.

[12] Belcastro, C. N. and J. V. Foster, "Aircraft loss-of-control accident analysis," Proceedings of AIAA Guidance, Navigation and Control Conference, AIAA2010-8004, Toronto, Canada, Aug., 2010.

[13] Calise, A. J., S. Lee, and M. Sharma, "Develpment of a reconfigurable flight control law for tailless aircraft," AIAA Journal of Guidance, Control, and Dynamics, vol. 25, no. 5, pp. 892-902, 2001.

[14] Campa, G., M. L. Fravolini, M. Napolitano, and B. Seanor, "Neural networksbased sensor validation for the flight control system of a B777 research model", Proceeding of American Control Conference, pp. 412-417, Anchorage, AK, 2002.

[15] Chen, W., M. Saif, "Adaptive actuator fault detection, isolation and accommodation (FDIA) in uncertain systems," International Journal of Control, vol. 80, no. 1, 2007, pp. 45-63.

[16] Deckert, J. C., M. N. Desai, J. J. Deyst, and A. S. Willsky, "F-8 DFBW sensor failure identification using analytic redundancy," IEEE Transactions on Automatic Control, vol. AC-22, no. 5, pp. 795-803, October 1977. 
[17] Demetriou, M. A. and M. M. Polycarpou, "Fault detection, diagnosis and accommodation of dynamical systems with actuator failures via on-line approximators", Proceeding of the American Control Conference, Philadelphia, Pennsylvania, June 1998, pp. 2879-2883.

[18] D. Deb, G. Tao, J. O. Burkholder, D. R. Smith, "Adaptive Synthetic Jet Actuator Compensation for A Nonlinear Aircraft Model at Low Angles of Attack," IEEE Transactions on Control Systems Technology, vol. 16, no. 5, pp. 983-995, September 2008.

[19] Dydek, Z. T., A. M. Annaswamy, and E. Lavretsky, "Adaptive control and the NASA X-15-3 flight revisited," Control Systems, vol. 30, no. 3, 2010, pp. 32-48.

[20] Elliott, H. and W. A. Wolovich, "A parameter adaptive control structure for linear multivariable systems," IEEE Transactions on Automatic Control, vol. AC-27, no. 5, pp. 340-352, 1982.

[21] Edmonds, J. and G. Hickman, "Damage detection and identification in composite aircraft components," Aerospace COnference Proceedings, 2000, Vol. 6, 18-25, pp. 263-269.

[22] Fortmann, T. E. and K. L. Hitz, An Introduction to Linear Control Systems, Marcel Dekker, New York, 1977.

[23] Golub, G. H. and C. F. van Loan, Matrix Computations, Johns Hopkins University Press, Baltimore, MD, 1983.

[24] Goodwin, G. C. and K. S. Sin, Adaptive Filtering Prediction and Control, Prentice-Hall, Englewood Cliffs, NJ, 1984.

[25] Grizzle, J. W., "Controlled invariance for discrete-time nonlinear systems with an application to the disturbance decoupling problem," IEEE Transactions on Automatic Control, vol. 30, no. 9, September 1985, pp. 868-874. 
[26] Grizzle, J. W., "On geometric approach for discrete time decoupling problems," Proceedings of 24th Conference on Decision and Control, Fort Lauderdale, Florida, 1985, pp. 366-370.

[27] Grizzle, J. W., P. V. Kokotovic, "Feedback linearization of sampled-data systems," IEEE Transactions on Automatic Control, vol. 33, no. 9, september 1988, pp. 857-859.

[28] Guo, T. H. and J. Nurre, "Sensor failure detection and recovery by neural networks", IJCNN-91-Seattle International Joint Conference on Neural Networks, pp.221-226, Seattle, 1991.

[29] Grizzle, J. W., "A linear algebraic framework for the analysis of discrete-time nonlinear systems", SIAM J. Control and Optimization, vol. 31, no. 4, July 1993, pp. 1026-1044.

[30] Gopinathan, M., J. D. Boskovic, R. K. Mehra, and C. Rago, "A multiple model predictive scheme for fault-tolerant flight control design," Proceedings of the 37th IEEE Conference on Decision and Control, pp. 1376-1381, 1998.

[31] Glavaski, S. and M. Elgersma, "Active aircraft fault detection and isolation," Autotestcon Proceedings of IEEE Systems Readiness Technology Conference, pp. 692-705, Valley Forge, PA, USA, Aug 2001.

[32] Gayaka, S. and B. Yao, "An adaptive robust scheme for multiple actuator fault accommodation," Proc. of ASME Dynamic Systems and Control Conference, Ann Arbor, Michigan, 2008, pp. 987-994.

[33] Guo, J. X., Y. Liu and G. Tao, "Multivariable MRAC with state feedback for output tracking," Proceedings of 2009 American Control Conference, pp. 592-597, St. Louis, MO, June 2009.

[34] Guo, J. X. and G. Tao, "A multivariable MRAC scheme with sensor uncertainty compensation," Proceedings of 48th IEEE Conference on Decision and Control, pp. 6632-6637, Shanghai, China, December 2009. 
[35] Guo, J. X., Y. Liu and G. Tao, "A Multivariable MRAC Design for Aircraft Systems under Failure and Damage Conditions," Proceedings of the 2011 American Control Conference, pp. 600-605, San Francisco, June 2011.

[36] Guo, J. X., G. Tao and Y. Liu, "A multivariable MRAC scheme with application to a nonlinear aircraft model," Automatica, vol. 47, no. 4, pp. 804-812, 2011.

[37] Guo, J. X., G. Tao, and Y. Liu, "Multivariable adaptive control of NASA generic transport aircraft model with damage," Journal of Guidance, Control, and Dynamics, vol. 34, no. 5, 2011, pp. 1495-1506.

[38] Guo, J. X. and G. Tao, "A feedback-based sensor uncertainty detection scheme," Proceedings of the 50th IEEE Conference on Decision and Control and European Control Conference, pp. 1122-1127, Orlando, FL, December 2011.

[39] Guo, J. X. and G. Tao, "Adaptive fault detection and fault-tolerant control of NASA generic transport aircraft model," Proceedings of the 2012 IFAC International Symposium on SAFEPROCESS, Mexico City, Mexico, August 2012.

[40] Huang C., J. Tylock, S. Engel, J. Withston, J. Eilbert, "Failure-accommodation neural networks flight control", Proceedings of the AIAA Guidance, Navigation and Control Conference, AIAA paper 92-4394, Hilton Head, SC, 1992.

[41] Hitachi, Y. and Hugh H. T. Liu, "Robust thrust-only control of damaged aircraft with horizontal tail loss," Proc. of AIAA Guidance, Navigation and Control Conference, 2010, AIAA-2010-7856.

[42] P. A. Ioannou and K. Tsakalis, "A robust direct adaptive controller," IEEE Transactions on Automatic Control, vol. AC-31, no. 11, pp. 1033-1043, 1986.

[43] Isidori, A., Nonlinear Control Systems, 3rd ed., Springer-Verlag, Berlin, 1995.2005.

[44] Ioannou, P. A. and J. Sun, Robust Adaptive Control, Prentice-Hall, Upper Saddle River, NJ, 1996. 
[45] Iserman, R. and P. Balle, "Trends in the application of model-based fault detection and diagnosis of technical processes," Control Engineering Practice, vol. 5, pp. 709-719, 1997.

[46] Imai, A. K., R. R. Costa, L. Hsu, G. Tao and P. V. Kokotović, "Multivariable MRAC using high frequency gain matrix factorization," Proc. of the 40th IEEE CDC, pp. 1193-1198, Orlando, FL, 2001.

[47] Isermann, R., "Model-based fault-detection and diagnosis-status and applications," Annual Reviews in Control, Vol. 29, Issue 1, pp. 71-85.

[48] Krstić, M., I. Kanellakopoulos and P. V. Kokotović, Nonlinear and Adaptive Control Design, John Wiley \& Sons, New York, 1995.

[49] Kim, B. S., A. J. Calise, "Nonlinear flight control using neural networks," AIAA Journal of Guidance, Control, and Dynamics, vol. 20, no. 1, pp. 26-33, 1997.

[50] Kazantzis, N. and C. Kravaris, "Time-discretization of nonlinear control systems via Taylor methods," Computers and Chemical Engineering, vol. 23, issue 6, pp. 763-784, 1999.

[51] Koh, B. H., P. Dharap, and S. Nagarajaiah, "Real-time structural damage monitoring by input error function", AIAA Journal, Vol. 43, No. 8, August 2005.

[52] Leitner, J., A. J. Calise and J. V. R. Prasad, "Analysis of adaptive neural networks for helicopter flight controls," AIAA Journal of Guidance, Control, and Dynamics, vol. 20, no. 5., pp. 972-979, September-October 1997.

[53] Landau, Y. D., R. Lozano and M. M'Saad, Adaptive Control, Springer, London, 1998.

[54] Liu, Y., and Tao, G., "Multivariable MRAC for aircraft with abrupt damages," Proceedings of 2008 American Control Conference, Seattle, WA, June 2008, pp. $2981-2986$. 
[55] Lavretsky, E., "Combined/composite model reference adaptive control," IEEE Transactions on Automatic Control, Vol. 54, No. 11, November 2009, pp. 26922697.

[56] Lavretsky, E., Gadient, R., and Gregory, I. M., "Predictor-based model reference adaptive control," Journal of Guidance, Control, and Dynamics, Vol. 33, No. 4, 2010, pp. 1195-1201.

[57] Liu, Y., Tao, G., and Joshi, S. M., "Modeling and model reference adaptive control of aircraft with asymmetric damages," Journal of Guidance, Control, and Dynamics, Vol. 33, No. 5, 2010.

[58] Morse, A. S., "Global stability of parameter adaptive control systems," IEEE Transactions on Automatic Control, vol. AC-25, no. 6, pp. 433-439, 1980.

[59] Morse, A. S., "Parametrizations for Multivariable Adaptive Control," Proc. 20th IEEE CDC, pp. 970-972, San Diego, CA, 1981.

[60] Monaco, S., D. Normand-Cyrot, and S. Stornelli, "On the linearizing feedback in nonlinear sampled data control schemes," Proceedings of 25th Conference on Decision and Control, Athens, Greece, December 1986, pp. 2056-2060.

[61] Monaco, S. and D. Normand-Cyrot, "Minimum-phase nonlinear discrete-time systems and feedback stabilization," Proceedings of the 26th Conference on Decision and Control, Los Angeles, CA, December 1987, pp. 979-986.

[62] Mutoh, Y. and P. N. Nikiforuk, "Inversed interactorizing and triangularizing with an arbitrary pole assignment using the state feedback," IEEE Transactions on Automatic Control, vol. 37, no. 5, 630-633, 1992.

[63] Marcos, A., S. Ganguli, and G. J. Balas, "An applicationi of $\mathrm{H}_{\infty}$ fault detection and isolation to a transport aircraft", Control Engineering Practice, 2005, pp. 105-119. 
[64] Murch, A.M., "A flight control system architecture for the NASA AirSTAR flight test infrastructure," AIAA Guidance, Navigation and Control Conference and Exhibit, Honolulu, Hawaii, Aug. 18-21, 2008.

[65] Narendra, K. S. and A. M. Annaswamy, Stable Adaptive Systems, Prentice-Hall, Englewood Cliffs, NJ, 1989.

[66] Naidu, S. R., E. Zafiriou, and T. J. McAvoy, "Use of neural networks for sensor failure detection in a control system", IEEE Control Systems Magazine, 10(3), pp. 49-55, 1990.

[67] Napolitano, M. R., C. I. Chen, and S. Naylor, "Aircraft failure detection and identification using neural networks", AIAA Journal of Guidance Control and Dynamics, 16(6), pp. 999-1009, 1993.

[68] Napolitano, M. R., C. D. Neppach, V. Casdorph, S. Naylor, M. Innocenti, and G. Silvestri, "A neural network based scheme for sensor failure detection, identification and accommodation", AIAA Journal of Guidance Control and Dynamics, 18(6), pp. 1280-1286, 1995.

[69] Nesic, D., A. R. Teel, and P. V. Kokotovic, "Sufficient conditions for stabilization of sampled-data nonlinear systems via discrete-time approximations," Systems and Control Letters vol. 38, issue 4-5, December 1999, pp. 259-270.

[70] Narendra, K. S. and J. Balakrishnan, "Adaptive control using multiple modes," IEEE Trans. on Automatic Control, vol. 42, no. 2, pp. 171-187, 1997.

[71] Nesic, D. and A. R. Teel, "A framework for stabilization of nonlinear sampleddata systems based on their approximate discrete-time models," IEEE Transactions on Automatic Control, vol. 49, no. 7, July 2004, pp. 1103-1122.

[72] Nguyen, N., Krishnakumar, K., Kaneshige, J., and Nespeca, P., "Flight dynamics and hybrid adaptive control of damaged aircraft," Journal of Guidance, Control, and Dynamics, Vol. 31, No. 3, 2008.pp, 751-764. 
[73] Ortega, R., L. Hsu and A. Astolfi, "Adaptive control of multivariable systems with reduced prior knowledge," Proceedings of the 40th IEEE Conference on Decision and Control, pp. 4198-4203, Orlando, FL, 2001.

[74] Rysdyk, R. T. and A. J. Calise, "Nonlinear adaptive flight control using neural networks," IEEE Control Systems Magazine, vol. 18, no. 6, December 1998.

[75] D. L. Raney, R. C. Montgomery, L. L. Green, and M. A. Park, "Flight control using distributed shape-change effector arrays," Proc. of the 41st AIAA/ASME/ASCE/AHS/ASC Structures, Structural Dynamics, and Materials Conference and Exhibit, AIAA-2000-1560, Atlanta.

[76] Sastry, S. and M. Bodson, Adaptive Control: Stability, Convergence, and Robustness, Prentice-Hall, Englewood Cliffs, NJ, 1989.

[77] Stengel, R.F., Flight Dynamics, Princeton University Press, 2004.

[78] Schwager, M. A., M. Annaswamy, and E. Lavretsky, "Adaptation-based reconfiguration in the presence of actuator failures and satuation," Proceedings of the 2005 American Control Conference, Portland, OR, June 2005.

[79] Sakellariou, J.S. and S.D. Fassois, "Vibration based fault detection and identification in an aircraft skeleton structure via a stochastic functional model based method", Mechanical Systems and Signal Processing, 22, 2008, pp. 557-573.

[80] Sonneveldt, L., van Oort, E. R., Chu, Q. P., de Visser, C. C., and Mulder, J. A., "Lyapunov-based fault tolerant flight control designs for a modern fighter aircraft model," Proc. of AIAA Guidance, Navigation, and Control Conference, No. AIAA-2009-6172, 2009.

[81] Q. Sang and G. Tao, "Multivariable state feedback for output tracking MRAC for piecewise linear systems," Proceedings of the 50th IEEE Conference on Decision and Control and European Control Conference, pp. 1784-1789, Orlando, FL, December 2011. 
[82] Tao, G., and Ioannou, P. A., "Robust model reference adaptive control fro multivariable plants," International Journal of Adaptive Control and Signal Processing, Vol. 2, No. 3, 1988, pp. 217-248.

[83] Tao, G. and P. V. Kokotović, Adaptive Control of Systems with Actuator and Sensor Nonlinearities, John Wiley \& Sons, New York, 1996.

[84] Tao, G., Adaptive Control Design and Analysis, John Wiley and Sons, New York, 2003.

[85] Tao, G., S. H. Chen, X. D. Tang and S. M. Joshi, Adaptive Control of Systems with Actuator Failures, Springer, March 2004.

[86] Trendafilova, I., "A study on vibration-based damage detection and location in an aircraft wing scaled model", Applied Mechanics and Materials, Vols, 3-4 (2005) pp. 309-314.

[87] Tancredi, D., Gu, Y., Phillips, K., Gururajan, S., and Napolitano, M. R., "Development of adaptive control laws for actuator fault accommodation," Proc. of AIAA Guidance, Navigation and Control Conference, 2010, AIAA-2010-7547.

[88] G. Tao and J. O. Burkholder, "Adaptive Actuator Nonlinearity Compensation for Multivariable Systems," Proceedings of 2011 AIAA Guidance, Navigation, and Control Conference, Paper AIAA-2011-6397, Portland, OR, August 2011.

[89] Wolovich, W. A. and P. L. Falb, "Invariants and canonical forms under dynamic compensation," SIAM J. Control and Optimization, vol. 14, no. 6, November 1976, pp. 996-1008.

[90] Weller, S. R. and G. C. Goodwin, "Hysteresis switching adaptive control of linear multivariable systems," IEEE Trans. on Automatic Control, vol. AC-39, no. 7 , pp. 1360-1375, 1994.

[91] Wise, K., J. S. Brinker, A. J. Calise, D. F. Enns and M. R. Elgersma, "Direct adaptive reconfigurable flight control for a tailless advanced fighter aircraft," Int. J. Robust and Nonlinear Control, vol. 9, pp. 999-1009, 1999. 
[92] Wang, D. and K.Y. Lum, "Adaptive unknown input observer approach for aircraft actuator fault detection and isolation," International Journal of Adaptive Control and Signal Processing, 2007, pp. 31-48.

[93] Yuz, J. I. and G. C. Goodwin, "On sampled-data models for nonlinear systems," IEEE Transactions on Automatic Control, vol.50, no. 10, Oct. 2005, pp. 14771489.

[94] Zhao, X., X. Ye, C. Zhang, and J. Sun, "Artificial neural network for sensor failure detection in an automotive engine", Proceeding of the Instrumental and Measurement Technology Conference, pp. 167-170, Hamamatsu, 1994.

[95] Zhang, Y. and J. Jiang, "Design of integrated fault detection, diagnosis and reconfigurable control systems," Proceeding of the 38th Conference on Decision and Control, Phoenix, Arizona, USA, December 1999, pp. 3578-3592.

[96] Zhang, X., T. Parisini, and M. Polycarpou, "Adaptive fault-tolerant control of nonlinear uncertain systems: an information-based diagnostic approach," IEEE Trans. on Automatic Control, vol. 49, no. 8, 2004, pp. 1259-1274.

[97] Zhang, Y. M., and J. Jiang, "Bibliographical review on reconfigurable faulttolerant control systems," IFAC Annual Reviews in Control, vol. 32, no. 2, 2008, pp. 229-252.

[98] Zhang, Z. and W. Chen, "Adaptive output feedback control of nonlinear systems with actuator failures," information Sciences, vol. 179, no. 24, pp. 4249-4260, 2009. 